\begin{abstract}
MA, JEONG BEOM. Modeling of Nanoindentation and Microstructural Ductile Behavior in Metallic Material Systems. (Under the direction of Professor Mohammed A. Zikry)

A new hierarchical computational method has been developed and used with a dislocation-density multiple-slip crystalline formulation, and a specialized grain-boundary (GB) kinematic scheme to understand and predict how nanoindentation affects ductile behavior in face centered cubic crystalline (f.c.c.) aggregates at scales that span the molecular to the continuum level in ductile crystalline systems. The hierarchical approach is based on using displacement profiles from molecular dynamics (MD) simulations of nanoindentation to obtain scaling relations, which are then related to the strains pertaining to indented depths. These strains, which are scale invariant, and the scaling factors related to different indented depths, grain-sizes, and grain aggregate sizes are then used in a microstructural finite-element method (FEM) formulation that accounts for dislocation-density evolution, dislocationdensity interactions with different GB orientations and distributions, crystalline structures, and grain-sizes. The accuracy of this hierarchical computational scheme was validated by comparing the predicted hardness values of crystalline gold with a number of experimental measurements, and the predictions accurately matched these measurements for different indented depths.
\end{abstract}

This approach provides a new methodology to link molecular level simulations, with a microstructurally-based FEM formulation that can be used to ascertain the effects of dislocation-density evolution, grain-sizes, GB orientations, and crystalline structure on nanoindented surfaces at temporal and spatial scales that are commensurate with 
microstructural behavior. In this study, different aggregates with different low and high angle GBs and grain-sizes were investigated. The interrelated effects of GB interfaces and orientations, dislocation-density impedances and transmissions through GB regions, and the loading and unloading of the nanoindenter were characterized as a function of how local hydrostatic pressures, accumulated plastic strains, dislocation-densities, and slip-system orienations evolved in critical indented regions. The activation and switching of different slip-systems due to local stress unloading were also predicted and characterized for all aggregates and GB orientations in terms of dislocation-densities and pressures at specific orientations and spatial positions within different grains and GB regions.

This validated hierarchical approach provides a predictive framework that can be used to design new experiments related to nanoindenation. But more importantly, it provides a general framework that can relate the transition of material behavior from the nano to the micro level, such that different phenomena and physical mechanisms can be accurately predicted from a nucleation threshold stage to a desired evolving microstructural mode. Hence, this can be used to potentially tailor crystalline aggregates over a broad spectrum of scales for desired applications at scales that span the nano to the micro levels. 


\title{
MODELING OF NANOINDENTATION AND MICROSTRUCTURAL DUCTILE BEHAVIOR IN METALLIC MATERIAL SYSTEMS
}

by

\section{JEONG BEOM MA}

A dissertation submitted to the Graduate Faculty of North Carolina State University in partial fulfillment of the requirements for the Degree of Doctor of Philosophy

\section{Mechanical Engineering}

\author{
Raleigh
}

2005

APPROVED BY:

J.S. Strenkowski, Ph.D.

L.M. Silverberg, Ph.D.
Z. Li, Ph.D.

M.A. Zikry, Ph.D.

Chair of Advisory Committee 


\section{DEDICATION}

I, Jeong Beom Ma, dedicate this research results to my father who always enlightens the road where I was, am, and will be, my mother, who devotes her endless cares and loves to my family, and me, my sister and brother who encourage me whenever, wherever, and whatever I did, my loving wife who takes care of my family with her loves, and is the best friend, and my dearest daughter, Haerim, who makes me smile at any time.

$$
\text { 나, 마정범은 이 연구결과를 }
$$

나의 과거와 현재 그리고 미래에 갈 길을 늘 밝혀주시는 나의 아버지와

나와 나의 가족을 위해 끝없는 보살핌과 사랑으로 헌신하신 나의 어머니와

내가 언제, 어디서, 무엇을 하던 항상 응원해 준 나의 형제자매와

나의 가족을 사랑으로 보살펴준 나의 친구와 같은 나의 아내와

그리고 나에게 항상 웃음을 선사하는 나의 사랑하는 딸 혜림에게

바칩니다. 


\section{BIOGRAPHY}

Jeong Beom Ma was born in Seoul, Korea on February 18, 1968, to Boksoon Park and Kyu-ha Ma. After completing his work at Sang-Moon High School, Seoul, Korea, in 1986, he entered the Hanyang University. In 1990, he received the degree of Bachelor of Science, with a major in Mechanical Engineering. In March of 1990, he entered the Graduate School of the Hanyang University. He was awarded the degree of Master of Science in Precision Mechanical Engineering in February of 1992. He joined with the Kia Motors Co. in December of 1991, and worked as a platform designer, layout developer, and crashworthiness improvement coordinator of platform body parts. In August of 1998, he came to the United States and enrolled at the University of Texas at Austin where he received his another degree of Master of Science in December of 2000 with a major in Aerospace Engineering. In August of 2001, he entered the Graduate School of the North Carolina State University, and he began working towards his Ph.D. degree in Mechanical Engineering. During his Ph.D. studies, he served as a teaching assistant and a research assistant on a Nanoscale Interdisciplinary Research Teams (NIRT) grant of National Science Foundation (NSF). 


\section{ACKNOWLEDGEMENT}

I am grateful for the support, guidance, and encouragement of my research advisor, mentor, and committee chair, Mohammed A. Zikry. He has spent countless hours teaching me to sort through my thinking in my own work and in the research I have worked on. His invaluable support and inspiration have had a major impact on this dissertation and will continue to shape my future work. I consider myself fortunate to have worked with him. I would also like to offer my special thanks to my committee members, John Strenkowski, Larry Silverberg, and Zhilin Li for their stimulating comments on this dissertation.

I am also thankful to Dr. Waeil Ashmawi, Omid Rezvanian, James Pearson, and Fadi Abdeljawad for their academic, emotional support and friendship. My friends, Young June Pyun, Dr. Changmock Shin, Woon Chang, Dr. Sanghyon Oh, and others who are not listed here whom I spent the 4 years in graduate school with will be never forgotten.

I am forever indebted to my parents, my sister and brother for their tremendous love and support over the years and for their confidence in me. I owe the deepest and most sincere thanks to my wife and best friend, Dr. Young Eun Chang for her devoted love and companionship. I also give my endless love to my precious daughter Haerim Ma. I would never have made it through graduate school without the support of my family. 


\section{TABLE OF CONTENTS}

Page

LIST OF TABLES …….................................................................................... vii

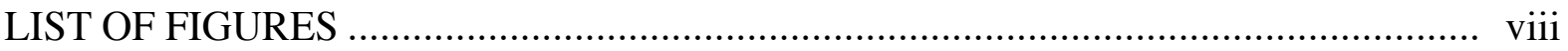

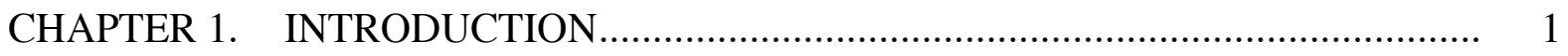

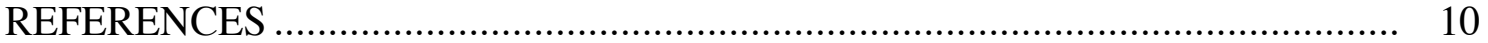

CHAPTER 2. DISLOCATION-DENSITY BASED MULTIPLE-SLIP CRYSTAL PLASTICITY CONSTITUTIVE FORMULATION.......................... 13

2.1 Dislocation-density Based Multiple-slip Crystal Plasticity Formulation ........... 13

2.2 Evolutionary Equations for the Mobile and Immobile Dislocation-densities..... 18

2.3 Determination of the Coefficients for the Coupled Evolutionary Equations ...... 24

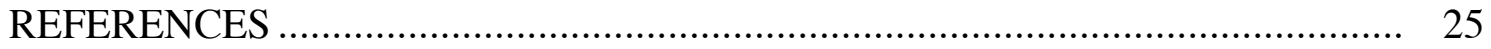

CHAPTER 3. NUMERICAL METHOD............................................................ 27

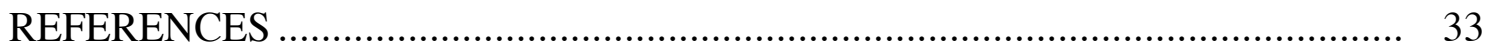

CHAPTER 4. GRAIN-BOUNDARY INTERFACIAL REGIONS AND INTERACTION MECHANISMS ....................................................... 34

4.1 Introduction .................................................................................... 34

4.2 Euler Space .................................................................................. 35

4.3 Kinematics of GB and Dislocation-density Interaction ................................. 38

4.4 GB Interaction Scheme ……................................................................. 39

4.5 Potential Dislocation-density Envelope ..................................................... 40

4.6 Dislocation-density Orientation and GB Coordinate Frame Transformation ..... 41

4.7 Slip-plane Geometric Compatibility …………........................................... 43

4.8 Transmission Distribution Factor ………………...................................... 45

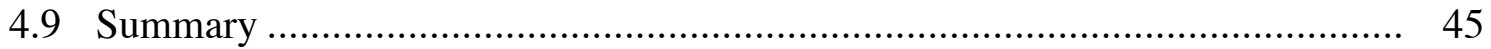

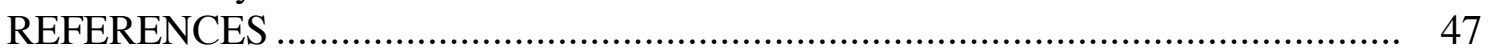

CHAPTER 5. HIERARCHICAL MODELING OF NANOINDENTATION OF POLYCRYSTALLINE GOLD AGGREGATES ................................... 53

5.1 Hierarchical Modeling of Nanoindentation: Linking and Scaling of Molecular Dynamic Simulations with the Microstructurally Based Finite-Element Formulation

5.1.1 Gold Crystalline Aggregate with Cube Orientation ....................... 54

5.1.2 Incorporation of Scaling Effects in Microstructural FEM Models ... 57

5.2 Validation of Hierarchical Modeling Scheme by Comparison of Hardness of Gold Single Crystals with Experimentally Obtained Values ......................... 59

5.3 Summary and Conclusions ...................................................................... 60 


\section{CHAPTER 6. NANOINDENTATION AND MICROSTRUCTURAL EVOLUTION} OF CUBE ORIENTED CRYSTALLINE AGGREGATES

6.1 Overall Aggregate Response and Slip-systems Activity during Global Loading 64

6.2 Immobile Dislocation-density Activities, Inelastic Behavior, and Local Stress and Pressure Response ....................................................... 66

6.3 Effects of Increased Indentation Depths …….............................................. 68

6.4 Effects of Larger-Sized Aggregate ............................................................ 71

6.5 Summary and Conclusions ................................................................... 74

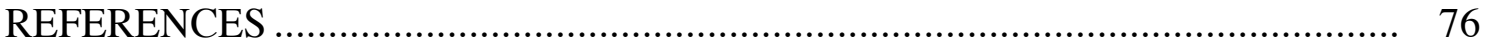

CHAPTER 7. NANOINDENTATION OF POLYCRYSTALLINE AGGREGATES WITH RANDOM LOW ANGLE GB MISORIENTATIONS.................. 90

7.1 Modeling of Polycrystalline Aggregates with Low Angle GBs ....................... 90

7.2 Grain-size Effects and Slip-systems Activities during Local Unloading............ 92

7.3 Immobile Dislocation-density Evolution, Plastic Strain, and Pressure Response: Model 1........................................................................... 93

7.4 Effects of Increased Indentation Depths: Model 1 ......................................... 94

7.5 Effects of Increased Aggregate Sizes: Model 2 ……...................................... 96

7.6 Crystalline Orientation Effects ................................................................... 97

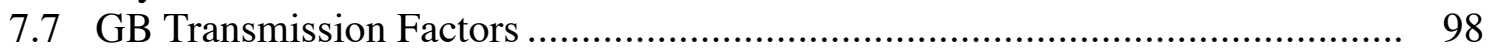

7.8 Summary and Conclusions ......................................................................... 98

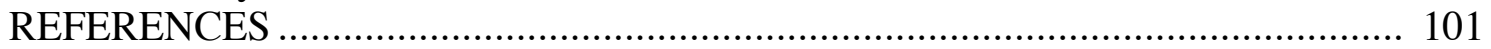

CHAPTER 8. RANDOM HIGH ANGLE GB EFFECTS ON NANOINDENTATION OF POLYCRYSTALLINE AGGREGATES......................................... 120

8.1 Grain-Size Effects and Slip-systems Activities during Local Unloading .......... 120

8.2 Immobile Dislocation-density Evolution, Plastic Strain, and Pressure Response: Model 1 ............................................................................. 121

8.3 Effects of High Strength GBs on Nanoindentation ......................................... 124

8.4 Crystalline Orientation Effects ............................................................... 126

8.5 GB Transmission Factors ......................................................................... 126

8.6 Summary and Conclusions .......................................................................... 127

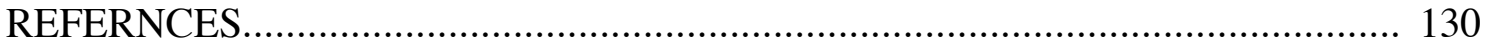

CHAPTER 9 RECOMMENDATIONS FOR FUTURE RESEARCH.......................... 148

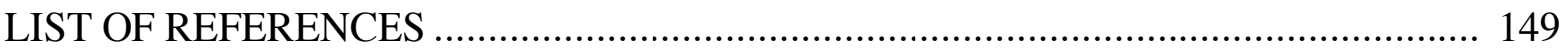




\section{LIST OF TABLES}

$\begin{array}{ll} & \text { Page }\end{array}$

Table 4.1. Slip-systems of f.c.c. metals ................................................... 38

Table 5.1. Material properties for gold single crystals ...................................... 55

Table 5.2. Scaling of MD simulations to microstructural FEM models .................... 56

Table 5.3. Grain-sizes and aggregates ................................................... 57

Table 5.4. Number of nodes applied normalized displacement input for different indentation depth .................................................................. 58

Table 6.1. Slip-system activity variation before and after local unloading ................ 66

Table 6.2. List of most active slip-systems at maximum normalized depths............. 66

Table 7.1. Slip-system activity variation before and after local load-drops during global loading (low angle GB misorientation)......................... 93

Table 7.2. List of most active slip-systems at maximum normalized depths.............. 93

Table 7.3. Immobile dislocation-density, plastic strains and pressure variation at final loading ........................................................................

Table 8.1. Slip-system activity variation before and after local load-drop during global loading (high angle GB misorientation)......................... 121

Table 8.2. List of most active slip-systems at maximum normalized depths.............. 122 


\section{LIST OF FIGURES}

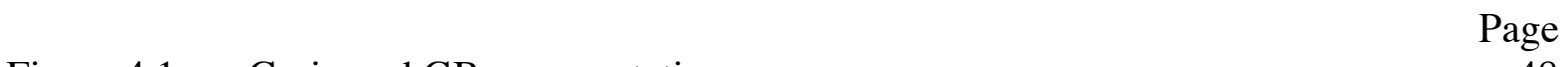

Figure 4.1. Grain and GB representation................................................ 48

Figure 4.2. Dislocation-density envelops ....................................................... 48

Figure 4.3. Schematic view for GB coordinates frame transformation .................... 49

Figure 4.4. Dislocation-density sign convention along the four GBs ..................... 50

Figure 4.5. General slip-plane geometric compatibility....................................... 51

Figure 4.6. Slip-plane $\theta$-compatibility .......................................................... 51

Figure 4.7. Slip-plane $\beta$-compatibility ..................................................... 52

Figure 5.1. Schematic model for finite-element analysis (scaling MD to FEA) .......... 62

Figure 5.2. Simplified loading-unloading curve for FEA .................................... 62

Figure 5.3. Schematic of indentation according to indenter size and indentation depth 63

Figure 5.4. Hardness of gold single crystals by FEA ....................................... 63

Figure 6.1. Stress-strain curves for gold single crystals with different normalized indented depths and aggregate sizes (a) $9 \%$ (b) $12.8 \%$ (c) $23.1 \% \ldots \ldots \ldots \ldots . . .77$

Figure 6.2. Immobile dislocation-density at final loading step for system 6 and 12 normalized by saturation (a), total immobile dislocation-density at final loading step non-normalized (b), just before unloading step for system 6 and 12 normalized by saturation (c), and total immobile dislocationdensity just before unloading step non-normalized (d). (Model 1 with 9\% indentation)

Figure 6.3. Plastic strain accumulation at final loading step (a), just before unloading step (b), pressure at final loading step normalized by yield stress (c), and pressure just before unloading step normalized by yield stress (d). (Model 1 with 9\% indentation)

Figure 6.4. Immobile dislocation-density at final loading step for system 2 and 8 normalized by saturation (a), total immobile dislocation-density at final loading step non-normalized (b), just before unloading step for system 2 and 8 normalized by saturation (c), and total immobile dislocationdensity just before unloading step non-normalized (d).

(Model 1 with $12.8 \%$ indentation) 
Figure 6.5. Plastic strain accumulation at final loading step (a), just before unloading step (b), pressure at final loading step normalized by yield stress (c), and pressure just before unloading step normalized by yield stress (d).

Model 1 with $12.8 \%$ indentation)

Figure 6.6. Immobile dislocation-density at final loading step for system 3, 4, 9, and 10 normalized by saturation (a), total immobile dislocation-density at final loading step non-normalized (b), just before unloading step for system 3, 4, 9, and 10 normalized by saturation (c), and total immobile dislocation-density just before unloading step non-normalized (d).

(Model 1 with $23.1 \%$ indentation)

Figure 6.7. Plastic strain accumulation at final loading step (a), just before unloading step (b), pressure at final loading step normalized by yield stress (c), and pressure just before unloading step normalized by yield stress (d). (Model 1 with $23.1 \%$ indentation)

Figure 6.8. Immobile dislocation-density at final loading step for system 2 and 8 normalized by saturation (a), total immobile dislocation-density at final loading step non-normalized (b), just before unloading step for system 2 and 8 normalized by saturation (c), and total immobile dislocationdensity just before unloading step non-normalized (d). (Model 2 with 9\% indentation)

Figure 6.9. Plastic strain accumulation at final loading step (a), just before unloading step (b), pressure at final loading step normalized by yield stress (c), and pressure just before unloading step normalized by yield stress $(\mathrm{d})$. (Model 2 with 9\% indentation)

Figure 6.10. Immobile dislocation-density at final loading step for system 1 and 7 normalized by saturation (a), total immobile dislocation-density at final loading step non-normalized (b), just before unloading step for system 1 and 7 normalized by saturation (c), and total immobile dislocationdensity just before unloading step non-normalized (d). (Model 2 with $12.8 \%$ indentation)

Figure 6.11. Plastic strain accumulation at final loading step (a), just before unloading step (b), pressure at final loading step normalized by yield stress (c), and pressure just before unloading step normalized by yield stress (d). (Model 2 with $12.8 \%$ indentation)

Figure 6.12. Immobile dislocation-density at final loading step for system 6 and 12 normalized by saturation (a), total immobile dislocation-density at final loading step non-normalized (b), just before unloading step for system 6 and 12 normalized by saturation (c), and total immobile dislocationdensity just before unloading step non-normalized (d). 
Figure 6.13. Plastic strain accumulation at final loading step (a), just before unloading step (b), pressure at final loading step normalized by yield stress (c), and pressure just before unloading step normalized by yield stress (d). (Model 2 with $23.1 \%$ indentation)

Figure 7.1. Polycrystalline aggregate, stress axis, and random low angle GB misorientations used in FEA

Figure 7.2. Stress-strain curves for gold polycrystalline aggregates with different normalized indented depths, aggregates sizes, and low angle misorientations (a) $9 \%$ (b) $12.8 \%$ (c) $23.1 \%$

Figure 7.3. Immobile dislocation-density at final loading step for system 5 normalized by saturation (a), total immobile dislocation-density at final loading step non-normalized (b), just before unloading step for system 5 normalized by saturation (c), and total immobile dislocation-density just before unloading step non-normalized (d). (Model 1 with 9\% indentation)

Figure 7.4. Plastic strain accumulation at final loading step (a), just before unloading step (b), pressure at final loading step normalized by yield stress (c), and pressure just before unloading step normalized by yield stress (d). (Model 1 with 9\% indentation)

Figure 7.5. Immobile dislocation-density at final loading step for system 5 normalized by saturation (a), total immobile dislocation-density at final loading step non-normalized (b), just before unloading step for system 5 normalized by saturation (c), and total immobile dislocation-density just before unloading step non-normalized (d). (Model 1 with $12.8 \%$ indentation).....

Figure 7.6. Plastic strain accumulation at final loading step (a), just before unloading step (b), pressure at final loading step normalized by yield stress (c), and pressure just before unloading step normalized by yield stress (d). (Model 1 with $12.8 \%$ indentation)

Figure 7.7. Immobile dislocation-density at final loading step for system 12 normalized by saturation (a), total immobile dislocation-density at final loading step non-normalized (b), just before unloading step for system 12 normalized by saturation (c), and total immobile dislocation-density just before unloading step non-normalized (d). (Model 1 with $23.1 \%$ indentation).

Figure 7.8. Plastic strain accumulation at final loading step (a), just before unloading step (b), pressure at final loading step normalized by yield stress (c), and pressure just before unloading step normalized by yield stress $(\mathrm{d})$. (Model 1 with $23.1 \%$ indentation) 
Figure 7.9. Immobile dislocation-density at final loading step for system 3 normalized by saturation (a), total immobile dislocation-density at final loading step non-normalized (b), just before unloading step for system 3 normalized by saturation (c), and total immobile dislocation-density just before unloading step non-normalized (d). (Model 2 with 9\% indentation)

Figure 7.10. Plastic strain accumulation at final loading step (a), just before unloading step (b), pressure at final loading step normalized by yield stress (c), and pressure just before unloading step normalized by yield stress (d). (Model 2 with 9\% indentation)

Figure 7.11. Three angular directions with respect to the indentation-loading axis

Figure 7.12. Immobile dislocation-density distribution along the different angles with respect to loading axis at final loading -15 grains and low angle GB orientation; (a) $\pm 30^{\circ}$ (GB: $\left.\mathrm{r} / \mathrm{h}= \pm 0.3569\right)$, (b) $\pm 45^{\circ}$ (GB: $\mathrm{r} / \mathrm{h}=0.2312$ and 0.2584 ), (c) $\pm 60^{\circ}$ (GB: $\mathrm{r} / \mathrm{h}=0.185$ ) (i) $9 \%$, (ii) $12.8 \%$, and (iii) $23.1 \%$

Figure 7.13. Plastic strain distribution along the different angles with respect to loading axis at final loading - 15 grains and low angle GB orientation;

(a) $\pm 30^{\circ}(\mathrm{GB}: \mathrm{r} / \mathrm{h}= \pm 0.3569)$, (b) $\pm 45^{\circ}(\mathrm{GB}: \mathrm{r} / \mathrm{h}=0.2312$ and 0.2584$)$, (c) $\pm 60^{\circ}(\mathrm{GB}: \mathrm{r} / \mathrm{h}=0.185$ ) (i) $9 \%$, (ii) $12.8 \%$, and (iii) $23.1 \%$

Figure 7.14. Normalized pressure distribution along the different angles with respect to loading axis at final loading - 15 grains and low angle GB orientation; (a) $\pm 30^{\circ}(\mathrm{GB}: \mathrm{r} / \mathrm{h}= \pm 0.3569)$, (b) $\pm 45^{\circ}(\mathrm{GB}: \mathrm{r} / \mathrm{h}=0.2312$ and 0.2584$),(\mathrm{c}) \pm 60^{\circ}$ (GB: $\mathrm{r} / \mathrm{h}=0.185$ ) (i) $9 \%$, (ii) $12.8 \%$, and (iii) $23.1 \%$

Figure 7.15. Transmission factor at GBs under the virtual indenter: model 1 with $9 \%$ loading (a), (b): TR for (111), and (c), (d): TR for (111), and (e), (f): TR for $(1 \overline{1} 1)$, and $(\mathrm{g}),(\mathrm{h})$ : TR for $(11 \overline{1})$

Figure 8.1. Stress-strain curves for gold polycrystalline aggregates with different normalized indented depths, aggregates sizes, and high angle misorientations (a) $9 \%$ (b) $12.8 \%$

Figure 8.2. Immobile dislocation-density at final loading step for system 4 normalized by saturation (a), total immobile dislocation-density at final loading step non-normalized (b), just before unloading step for system 4 normalized by saturation (c), and total immobile dislocation-density just before unloading step non-normalized (d). (Model 1 with 9\% indentation)

Figure 8.3. Plastic strain accumulation at final loading step (a), just before unloading step (b), pressure at final loading step normalized by yield stress (c), and pressure just before unloading step normalized by yield stress (d). (Model 1 with 9\% indentation) 
Figure 8.4. Immobile dislocation-density at final loading step for system 5 normalized by saturation (a), total immobile dislocation-density at final loading step non-normalized (b), just before unloading step for system 5 normalized by saturation (c), and total immobile dislocation-density just before unloading step non-normalized (d). (Model 1 with $12.8 \%$ indentation). 134

Figure 8.5. Plastic strain accumulation at final loading step (a), just before unloading step (b), pressure at final loading step normalized by yield stress (c), and pressure just before unloading step normalized by yield stress (d). (Model 1 with $12.8 \%$ indentation)

Figure 8.6. Immobile dislocation-density at final loading step for system 11 normalized by saturation (a), total immobile dislocation-density at final loading step non-normalized (b), just before unloading step for system 11 normalized by saturation (c), and total immobile dislocation-density just before unloading step non-normalized (d). (Model 2 with $9 \%$ indentation) 136

Figure 8.7. Plastic strain accumulation at final loading step (a), just before unloading step (b), pressure at final loading step normalized by yield stress (c), and pressure just before unloading step normalized by yield stress (d). (Model 2 with 9\% indentation)

Figure 8.8. Immobile dislocation-density at final loading step for system 10 normalized by saturation (a), total immobile dislocation-density at final loading step non-normalized (b), just before unloading step for system 10 normalized by saturation (c), and total immobile dislocation-density just before unloading step non-normalized (d). (Model 2 with $12.8 \%$ indentation).....

Figure 8.9. Plastic strain accumulation at final loading step (a), just before unloading step (b), pressure at final loading step normalized by yield stress (c), and pressure just before unloading step normalized by yield stress $(\mathrm{d})$. (Model 2 with $12.8 \%$ indentation)

Figure 8.10. Immobile dislocation-density at final loading step for system 1 normalized by saturation (a), total immobile dislocation-density at final loading step non-normalized (b), just before unloading step for system 1 normalized by saturation (c), and total immobile dislocation-density just before unloading step non-normalized (d). (Model 3 with 9\% indentation and stronger GBs) 140

Figure 8.11. Plastic strain accumulation at final loading step (a), just before unloading step (b), pressure at final loading step normalized by yield stress (c), and pressure just before unloading step normalized by yield stress (d). (Model 3 with 9\% indentation and stronger GBs)

Figure 8.12. Immobile dislocation-density at final loading step for system 3 normalized by saturation (a), total immobile dislocation-density at final loading step 
non-normalized (b), just before unloading step for system 3 normalized by saturation (c), and total immobile dislocation-density just before unloading step non-normalized (d). (Model 4 with 9\% indentation and stronger GBs) 142

Figure 8.13. Plastic strain accumulation at final loading step (a), just before unloading step (b), pressure at final loading step normalized by yield stress (c), and pressure just before unloading step normalized by yield stress $(\mathrm{d})$. (Model 4 with 9\% indentation and stronger GBs)

Figure 8.14. Immobile dislocation-density distribution along the different angles with respect to loading axis at final loading -15 grains and high angle GB orientation; (a) $\pm 30^{\circ}$ (GB: $\left.\mathrm{r} / \mathrm{h}= \pm 0.3569\right)$, (b) $\pm 45^{\circ}(\mathrm{GB}: \mathrm{r} / \mathrm{h}=0.2312$ and 0.2584 ), (c) $\pm 60^{\circ}$ (GB: $\mathrm{r} / \mathrm{h}=0.185$ ) (i) $9 \%$, and (ii) $12.8 \%$

Figure 8.15. Plastic strain distribution along the different angles with respect to loading axis at final loading - 15 grains and high angle GB orientation;
(a) $\pm 30^{\circ}$ (GB: $\left.\mathrm{r} / \mathrm{h}= \pm 0.3569\right)$, (b) $\pm 45^{\circ}$ (GB: $\mathrm{r} / \mathrm{h}=0.2312$ and 0.2584$)$, (c) $\pm 60^{\circ}$ (GB: $\mathrm{r} / \mathrm{h}=0.185$ ) (i) $9 \%$, and (ii) $12.8 \%$

Figure 8.16. Normalized pressure distribution along the different angles with respect to loading axis at final loading -15 grains and high angle GB orientation; (a) $\pm 30^{\circ}(\mathrm{GB}: \mathrm{r} / \mathrm{h}= \pm 0.3569)$, (b) $\pm 45^{\circ}(\mathrm{GB}: \mathrm{r} / \mathrm{h}=0.2312$ and 0.2584$),(\mathrm{c}) \pm 60^{\circ}$ (GB: $\mathrm{r} / \mathrm{h}=0.185$ ) (i) $9 \%$, and (ii) $12.8 \%$

Figure 8.17. Transmission factor at GBs under the virtual indenter: model 1 with $9 \%$ loading (a), (b): TR for (111), and (c), (d): TR for (111), and (e), (f): TR for $(1 \overline{1} 1)$, and $(\mathrm{g}),(\mathrm{h})$ : TR for $(11 \overline{1})$ 


\section{CHAPTER 1}

\section{INTRODUCTION}

The synthesis, processing, analysis, and design of nanostructured materials require accurate material properties and a detailed understanding of material behavior at different physical scales. As nanocrystalline grain-sizes are refined, one of the few available experimental techniques is nanoindentation, which can be utilized to determine material hardness and elastic and inelastic response. However, reliable nanoindentation measurements of material properties of nanocrystalline materials have been severely hampered by an inability to account for different physical mechanisms, such as grain-boundary (GB) effects, dislocation activities, and GB sliding that span different scales. Numerous studies have clearly indicated that inelastic behavior is difficult to determine by nanoindenation due to localized dislocation activities over small volumes that are dominated by a large fraction of GB surfaces. Furthermore, crystalline aggregates, which can be comprised of a collection of nanograins, can approach micro scales. Hence, the transition from the nano to the micro level behavior needs to be better understood and accurately predicted, such that indentation can be used to potentially tailor crystalline aggregates for desired applications.

The accurate measurement of the hardness of bulk and thin film crystalline materials, such as gold and copper, is critical due to the utility of these crystalline materials as coatings and conductors for semiconductor, microelectronic, and biomedical applications. Gane and Bowden (1968) used a scanning electron microscope (SEM) and microindentation to determine the microstrength and microdeformation of gold, copper, and aluminum crystals. Oliver and Pharr (1992) obtained the indentation load-displacement behavior of six 
materials-fused silica, soda-lime glass, and single crystals of aluminum, tungsten, quartz, and sapphire-with a Berkovich indenter to establish a method for determining hardness and elastic modulus. They found that the load-displacement curves during unloading are not linear, even in the initial stages, which indicates that the material response is inelastic almost from the onset of indentation. They accounted for the curvature in the unloading data by presenting it as power-law relation between the loads and displacements. They also provided a physically justifiable procedure for determining the depth that should be used in conjunction with the indenter shape function to establish the contact area at the peak load, which is essential for calculating the hardness. Corcoran, Colton et al. (1997) obtained reproducible displacement profiles near the theoretical yield strength for pure gold single crystals in agreement with dislocation nucleation.

Li, Nardi et al. (2005) used the atomic force microscope (AFM) to investigate the hardness of gold thin films by applying the projected area calibration, and found that the hardness is $2.1 \pm 0.3 \mathrm{GPa}$ for gold single crystals. Gerberich, T ymiak et al. (2002) experimentally studied the hardness of several materials including gold single crystals, and found that it decreased with increasing depth irrespective of increasing or decreasing strain gradients. Smith and Zheng (2000) investigated glass, gold, and single crystal silicon at room temperature and at $200^{\circ} \mathrm{C}$ by using a depth sensing indentation instrument, and they concluded that the hardness value decreased at higher temperature.

Interfacial Force Microscope (IFM) has also been used in conjunction with nanoindentation. Michalske and Houston (1998) used IFM to investigate the nano-scale elastic and plastic response of gold single crystal (111) surfaces by using controlled-probe contacts with different radii. They found that the stress at the plastic threshold increases with 
decreasing probe-tip radius. Kiely and Houston (1998) and Kiely, Hwang et al. (1998) extended these experiments to (110) and (001) surfaces of gold single crystals, and determined the elastic indentation modulus variation for different surfaces and the maximum resolved shear stress of $1.8 \mathrm{GPa}$ for plastic deformation. Due to the effects of surface steps on the initiation of plastic yield, the mean stress at yield was $30 \%$ to $45 \%$ lower at a step than in regions free of surface defects. Kiely, Jarausch et al. (1999) observed two distinct regimes of plastic deformation, which are distinguished by the magnitude of discontinuities in load relaxation. At lower stresses, relaxation occurs in small deviations from elastic behavior, while at the higher stresses they take the form of large load drops, often resulting in complete relaxation of the applied load. These major events create a relatively wide plastic zone that subsequently deepens more rapidly than it widens. Tawara, Matsukawa et al. (2003) investigated the effect of deformation speed on defect structures introduced into bulk gold specimens at room temperature, over a wide range of strain rates from $\dot{\varepsilon}=10^{-2} \sim 10^{6} s^{-1}$. As the strain rate increased, dislocation structure changed from heterogeneous distributions, socalled cell structure, to random distributions. High-speed deformation induces an abnormal mechanism of plastic deformation, which falls beyond the scope of dislocation theory.

Embedded-atom method (EAM) and other MD-based techniques have been used to predict nucleation phenomena, such as dislocation formation and GB sliding. Kreuzer and Pippan (2004) used MD simulations to model nanoindentation by means of dislocation movement. They found that the nominal hardness of the material is mainly influenced by the critical shear stress necessary to activate the dislocation sources, whereas the decrease of the hardness at small indentation depth is dependent on the lattice friction stress for the dislocation movement. Schall and Brenner (2004) determined by MD simulations, based on 
the embedded atom method (EAM), how small variations in the contact area during plastic indentation of $\mathrm{Au}$ (111) substrate under an applied in-plane stress can dramatically affect calculated hardness values. Kelchner, Plimpton et al. (1998); Zimmerman, Kelchner et al. (2001); Knap and Ortiz (2003); Greer and Nix (2005); and Greer, Oliver et al. (2005) have used MD techniques to investigate the gold surfaces and aggregates. These investigations have generally provided atomistic imaging of dislocation nucleation during displacementcontrolled indentation on a passivated surface. Kelchner, Plimpton et al. (1998) has shown that for $\mathrm{Au}$ (111) surface, nucleation of partial dislocation loops occurs below the surface inside the indenter contact area. These predictions indicate that while the load needed to nucleate dislocations decreases significantly when indenting close to the step, the extent of the step's influence is not as great as seen experimentally (Zimmerman, Kelchner et al., 2001). The dislocation structures for the large indenter are found to be less sharp, and to extend over a larger region than for the small indenter. Furthermore, the large-indenter forcedisplacement curve differs from that corresponding to the small indenter in one important respect, which is the absence of force drops during indentation, despite profuse dislocation activity (Knap and Ortiz, 2003). Microstructural inhomogeneities across the surface of gold films lead to position-dependent nanoindentation behavior. The crystals exhibit strong size effects at the sub-micron scale (Greer, Oliver et al., 2005). Atomistic simulations (Greer and Nix, 2005) suggest that the yield strength depends on the sample size even without strain gradients, and it scales with the sample size through a power relationship. Greer and Nix (2005) found that significant flow stresses of several GPa can occur in the indented region. These high strengths appear to be controlled by dislocation starvation or dislocation free zones, unique to small crystals. Hasnaoui, Derlet et al. (2004) undertook large-scale MD 
simulations of gold nanocrystalline. Their analysis provided an understanding of how dislocations emitted under an indenter, and how GB networks act as a sinks or as emitters of dislocations.

Other computational techniques have attempted to couple atomistic-based methods with continuum methods. Shilkrot, Curtin et al. (2002); Shilkrot, Miller et al. (2002); Miller, Shilkrot et al. (2004); Shilkrot, Miller et al. (2004) incorporated atomistic effects within a continuum entity, such that a single computational framework can be utilized. Near the indenter, an MD analysis, within a predefined region is utilized, and outside of this region, an FEM technique is utilized. They investigated how material hardness was size dependent, the role of dislocation dissociation on deformation, reverse plasticity including nucleation of dislocations on unloading and annihilation, permanent deformation including surface uplift after full unloading, the effects of film thickness on the load-displacement response, and the differences between displacement and force controlled loading. Jeng and Tan (2004) adopted an atomic-scale model based on nonlinear finite element formulation to analyze the stress and strain induced in a very thin film during the nanoindentation. Their results indicated that the microscopic plastic deformation in the thin film is caused by instability of its crystalline structure, and that the magnitude of the nanohardness varies with the maximum indentation depth and the geometry of the indenter. Choi, Krystyn et al. (2003) used FE modeling and MD simulations of nanoindentation for aluminum on the silicon substrate. They concluded that FE analyses have limited utility in rationalizing naoindentation experiments in which discrete discontinuities are dominant, and that MD simulations can exhibit some experimental observations. Knapp, Follstaedt et al. (2003) determined the yield strength, the elastic modulus, and layer hardness of nickel-based layers by applying detailed FE modeling 
of nanoindentation. Multiscale quasicontinuum models of plasticity developed by Phillps, Rodney et al. (1999) were used to elucidate the mechanisms of dislocation nucleation and interaction, and to illustrate how such information can be imported into explicit models of dislocation dynamics.

It is well understood that GB structure, orientation, and distributions are microstructural features that characterize the initiation and evolution of deformation and failure modes in crystalline metals, alloys, and intermetallics. As noted by Suresh, Nieh et al. (1999); Gerberich, Tymiak et al. (2002); Lilleodden, Zimmerman et al. (2003); and Hasnaoui, Derlet et al. (2004), one of the major challenges is determining how dislocation density nucleation, emission, transmission, and absorption within GB regions affect overall inelastic behavior for crystalline materials subjected to nanoindentation. Furthermore, investigations, by Matsukawa, Yasunaga et al. (2003), where TEM was used to characterize ductile fracture in nanoindeneted gold, found that an unusual microstructure, which contains vacancy-type point defect clusters of extraordinary high density near GB regions. These defect clusters were produced in local heavy deformation without dislocation motion. They suggested that the instability of crystalline state under tremendously high internal stress level is a key factor for the mechanism of dislocation-free plastic deformation near GB regions. Ohmura et al. (2004) investigated the effects of GBs on dislocation interactions in martenistic steels. In the case of a low-angle GBs, the dislocations induced by the indenter piled up against the boundary. As the indenter penetrated further, a critical stress appeared to have been reached, and a high density of dislocations was suddenly emitted on the far side of the GB into the adjacent grain. In the case of the high-angle GB, the numerous dislocations that were produced by the indentation were simply absorbed into the boundary, with no indication 
of pileup or the transmission of strain. They postulated that this markedly different behavior between low and high angle GBs is mainly due to high transmission of dislocations between grains for the high angle GB distribution.

Therefore, the physical scale on which to investigate different dislocation mechanisms involves different spatial and temporal levels that span the atomistic to the continuum levels. Atomistic methods are best suited for predictions related to defect nucleation, while microstructural and continuum-based methods are best suited for how defects evolve beyond the nucleation threshold stage and how several dislocations evolve to a population density. For example, as clearly indicated by the atomistic simulations of the nanoindenation of nanocrystalline gold by Derlet, Hasnaoui et al. (2003) and Feichtinger, Derlet et al. (2003), GBs act as efficient sinks for dislocation nucleation below the indenter and these sinks result in intergranular sliding. They also suggested that temporal and spatial scales of these modeling techniques precluded a detailed and accurate structural characterization of the simulated samples.

Hence, what these myriad investigations indicate is that nanoindentation triggers material mechanisms, such as slip-system activation and dislocation-density interactions and evolution due to crystalline structure, lattice rotation, and GB effects that cannot be predicted or tracked by atomistic-based methods. Atomistic-based methods, due to spatial and temporal limitations, can be used to predict and model how phenomena, such as the initiation of GB sliding and the initiation of dislocations and other defects, but other methods need to be used beyond the nucleation stage associated with defect formation. Furthermore, it is also evident, that microstructural effects, such as collective grain activities and GB effects that are near the indented surface play a critical role in the overall behavior. 
Hence, the primary purpose of this study is to be able to understand and predict how dislocation densities and inelastic deformation modes evolve beyond the defect nucleation stage. We propose a hierarchical computational model that spans the nano to the micro scales. This model is based on using displacement profiles, which were obtained from MD simulations of nanoindentation, and these displacement profiles are then used with specialized microstructural-based finite element formulations to track the evolution of dislocation densities and inhomogeneous deformation modes in crystalline aggregates with different grain-sizes and GB orientations. The hierarchical model was validated by predicting the hardness of gold, and comparing these predictions with different experimental observations and measurements.

An inelastic dislocation-density based multiple-slip crystalline constitutive formulation and an interfacial GB kinematic scheme was used, in conjunction with the hierarchical model, to obtain a detailed understanding and accurate prediction of interrelated local material mechanisms that control and affect inhomogeneous deformation modes in f.c.c. polycrystalline aggregates with random GB orientations and distributions. In this formulation, the length scale between multiple slip crystalline formulations and dislocation densities is bridged by coupling evolutionary equations for the mobile and immobile dislocation densities, through the temperature dependent flow stress and slip rates on each slip system, to a multiple slip rate dependent crystal plasticity formulation. The derivation of these evolutionary equations are based on accepted physical relations, and generally account for thermally activated dislocation activities such as generation, interaction, and annihilation that are generally representative of the dislocation structures in cubic crystalline metals (see, for example, Zikry and Kao, 1996). 
Most polycrystalline formulations generally do not account for GB effects such as dislocation density and slip transmission, blockage, and absorption. These effects could result due to GB orientation, structure or interfacial stress mismatches (see, for example, Zikry and Kao, 1996 and Ashmawi and Zikry, 2000). In this study, GB effects are accounted for by the introduction of interfacial regions that are used to track slip and dislocation density transmission and impedance at different GB interfaces. Therefore, we can track dislocationdensities within specific regions and orientations to determine how GB structure and orientation affects nanoindenation. These methodologies can provide guidelines for the design of experiments that can be used to track and monitor behavior in critical regions.

This dissertation is organized as follows: the rate-dependent dislocation-density based constitutive formulation is introduced in chapter 2; the finite-element method is outlined in chapter 3; the formulations for GB interfacial regions and interaction mechanisms are provided in chapter 4; the hierarchical scheme that links the MD simulations with the microstructural FEM method and the experimental validation are discussed in chapter 5; the microstructural evolution of the cube orientated aggregates is presented in chapter 6; aggregates with low and high angle GB distributions and orientations are discussed in chapter 7 and 8 ; recommendations for future research are outlined in chapter 9 . 


\section{References}

Ashmawi, W. M. and M. A. Zikry (2000). "Effects of grain boundaries and dislocation density evolution on large strain deformation modes in fcc crystalline materials." Journal of Computer-Aided Materials Design 7(1): 55-62.

Choi, Y., K. J. Van Vliet, et al. (2003). "Size effects on the onset of plastic deformation during nanoindentation of thin films and patterned lines." Journal of Applied Physics 94(9): 6050-6058.

Corcoran, S. G., R. J. Colton, et al. (1997). "Anomalous plastic deformation at surfaces: Nanoindentation of gold single crystals." Physical Review B 55(24): 16057-16060.

Derlet, P. M., A. Hasnaoui, et al. (2003). "Atomistic simulations as guidance to experiments." Scripta Materialia 49(7): 629-635.

Feichtinger, D., P. M. Derlet, et al. (2003). "Atomistic simulations of spherical indentations in nanocrystalline gold." Physical Review B 67(2).

Gane, N. and F. P. Bowden (1968). "Microdeformation of Solids." Journal of Applied Physics 39(3): 1432-\&.

Gerberich, W. W., N. I. Tymiak, et al. (2002). "Interpretations of indentation size effects." Journal of Applied Mechanics-Transactions of the Asme 69(4): 433-442.

Greer, J. R. and W. D. Nix (2005). "Size dependence of mechanical properties of gold at the sub-micron scale." Applied Physics a-Materials Science \& Processing 80(8): 16251629.

Greer, J. R., W. C. Oliver, et al. (2005). "Size dependence of mechanical properties of gold at the micron scale in the absence of strain gradients." Acta Materialia 53(6): 18211830.

Hasnaoui, A., P. M. Derlet, et al. (2004). "Interaction between dislocations and grain boundaries under an indenter - a molecular dynamics simulation." Acta Materialia 52(8): 2251-2258.

Jeng, Y. R. and C. M. Tan (2004). "Study of nanoindentation using FEM atomic model." Journal of Tribology-Transactions of the Asme 126(4): 767-774.

Kelchner, C. L., S. J. Plimpton, et al. (1998). "Dislocation nucleation and defect structure during surface indentation." Physical Review B 58(17): 11085-11088. 
Kiely, J. D. and J. E. Houston (1998). "Nanomechanical properties of Au (111), (001), and (110) surfaces." Physical Review B 57(19): 12588-12594.

Kiely, J. D., R. Q. Hwang, et al. (1998). "Effect of surface steps on the plastic threshold in nanoindentation." Physical Review Letters 81(20): 4424-4427.

Kiely, J. D., K. F. Jarausch, et al. (1999). "Initial stages of yield in nanoindentation." Journal of Materials Research 14(6): 2219-2227.

Knap, J. and M. Ortiz (2003). "Effect of indenter-radius size on Au(001) nanoindentation." Physical Review Letters 90(22).

Knapp, J. A., Follstaedt, D.M., and Myers, S.M. (2003). "Evaluating Micromechanical Properties at Surfaces using Nanoindentation with Finite-element Modeling." International Journal of Damage Mechanics 12(4): 377-387.

Kreuzer, H. G. M. and R. Pippan (2004). "Discrete dislocation simulation of nanoindentation." Computational Mechanics 33(4): 292-298.

Li, X. D., P. Nardi, et al. (2005). "Direct nanomechanical machining of gold nanowires using a nanoindenter and an atomic force microscope." Journal of Micromechanics and Microengineering 15(3): 551-556.

Lilleodden, E. T., J. A. Zimmerman, et al. (2003). "Atomistic simulations of elastic deformation and dislocation nucleation during nanoindentation." Journal of the Mechanics and Physics of Solids 51(5): 901-920.

Matsukawa, Y., K. Yasunaga, et al. (2003). "Dynamic observation of dislocation-free plastic deformation in gold thin foils." Materials Science and Engineering a-Structural Materials Properties Microstructure and Processing 350(1-2): 8-16.

Michalske, T. A. and J. E. Houston (1998). "Dislocation nucleation at nano-scale mechanical contacts." Acta Materialia 46(2): 391-396.

Miller, R. E., L. E. Shilkrot, et al. (2004). "A coupled atomistics and discrete dislocation plasticity simulation of nanoindentation into single crystal thin films." Acta Materialia 52(2): 271-284.

Ohmura, T., A. M. Minor, et al. (2004). "Dislocation-grain boundary interactions in martensitic steel observed through in situ nanoindentation in a transmission electron microscope." Journal of Materials Research 19(12): 3626-3632. 
Oliver, W. C. and G. M. Pharr (1992). "An Improved Technique for Determining Hardness and Elastic-Modulus Using Load and Displacement Sensing Indentation Experiments." Journal of Materials Research 7(6): 1564-1583.

Phillips, R., D. Rodney, et al. (1999). "Hierarchical models of plasticity: dislocation nucleation and interaction. " Modeling and Simulation in Materials Science and Engineering 7(5): 769-780.

Schall, J. D. and D. W. Brenner (2004). "Atomistic simulation of the influence of pre-existing stress on the interpretation of nanoindentation data." Journal of Materials Research 19(11): 3172-3180.

Shilkrot, L. E., W. A. Curtin, et al. (2002). "A coupled atomistic/continuum model of defects in solids." Journal of the Mechanics and Physics of Solids 50(10): 2085-2106.

Shilkrot, L. E., R. E. Miller, et al. (2002). "Coupled atomistic and discrete dislocation plasticity." Physical Review Letters 89(2).

Shilkrot, L. E., R. E. Miller, et al. (2004). "Multiscale plasticity modeling: coupled atomistics and discrete dislocation mechanics." Journal of the Mechanics and Physics of Solids 52(4): 755-787.

Smith, J. F. and S. Zheng (2000). "High temperature nanoscale mechanical property measurements." Surface Engineering 16(2): 143-146.

Suresh, S., T. G. Nieh, et al. (1999). "Nano-indentation of copper thin films on silicon substrates." Scripta Materialia 41(9): 951-957.

Tawara, T., Y. Matsukawa, et al. (2003). "Defect structure of gold introduced by high-speed deformation." Materials Science and Engineering a-Structural Materials Properties Microstructure and Processing 350(1-2): 70-75.

Zikry, M. A. and M. Kao (1996). "Dislocation based multiple-slip crystalline constitutive formulation for finite-strain plasticity." Scripta Materialia 34(7): 1115-1121.

Zikry, M. A. and M. Kao (1996). "Inelastic microstructural failure mechanisms in crystalline materials with high angle grain boundaries." Journal of the Mechanics and Physics of Solids 44(11): 1765-1798.

Zimmerman, J. A., C. L. Kelchner, et al. (2001). "Surface step effects on nanoindentation." Physical Review Letters 8716(16): art. no.-165507. 


\section{CHAPTER 2}

\section{DISLOCATION-DENSITY BASED MULTIPLE-SLIP CRYSTAL PLASTICITY CONSTITUTIVE FORMULATION}

In this chapter, the formulation for the multiple-slip crystal plasticity rate-dependent constitutive relations, and the derivation of the evolutionary equations for the mobile and immobile dislocation-densities, which are coupled to the multiple-slip crystalline formulation, are presented.

\subsection{Dislocation-density Based Multiple-slip Crystal Plasticity Formulation}

The crystal plasticity constitutive framework used in this study is based on the formulation developed in Zikry and Kao (1996), Kameda and Zikry (1998). In that formulation, it has been assumed that the deformation gradient can be decomposed into elastic and inelastic components. The velocity gradient tensor, $V_{i, j}$, is related to the deformation gradient by

$$
V_{i, j}=\dot{F}_{i j} F_{j i},
$$

where $F_{i j}$ is the total deformation gradient. And decomposing the velocity gradient tensor, $V_{i, j}$, into its symmetric and skew-symmetric parts as

$$
V_{i, j}=D_{i j}+W_{i j}
$$


where $D_{i j}$ is the total deformation rate tensor or stretching tensor,

$$
D_{i j}=\frac{1}{2}\left(V_{i, j}+V_{j, i}\right)
$$

and $W_{i j}$ is the total spin tensor or vorticity tensor,

$$
W_{i j}=\frac{1}{2}\left(V_{i, j}-V_{j, i}\right)
$$

The total deformation rate tensor, $D_{i j}$, and the total spin tensor, $W_{i j}$, are then each additively decomposed into elastic and plastic components as

$$
\begin{aligned}
& D_{i j}=D_{i j}^{*}+D_{i j}^{P}, \\
& W_{i j}=W_{i j}^{*}+W_{i j}^{P},
\end{aligned}
$$

where $W_{i j}$ includes the rigid body spin. The inelastic parts are defined in terms of the crystallographic slip-rates as

$$
\begin{aligned}
& D_{i j}^{P}=P_{i j}^{(\alpha)} \dot{\gamma}^{(\alpha)}, \\
& W_{i j}^{P}=\omega_{i j}^{(\alpha)} \dot{\gamma}^{(\alpha)},
\end{aligned}
$$


where $\alpha$ is summed over all slip-systems, and the tensors $P_{i j}^{(\alpha)}$ and $\omega_{i j}^{(\alpha)}$ are symmetric and skew-symmetric second-order tensors, and are defined in terms of the unit normal and the unit slip vectors as

$$
\begin{aligned}
& P_{i j}^{(\alpha)}=\frac{1}{2}\left(s_{i}^{(\alpha)} n_{j}^{(\alpha)}+s_{j}^{(\alpha)} n_{i}^{(\alpha)}\right), \\
& \omega_{i j}^{(\alpha)}=\frac{1}{2}\left(s_{i}^{(\alpha)} n_{j}^{(\alpha)}-s_{j}^{(\alpha)} n_{i}^{(\alpha)}\right),
\end{aligned}
$$

where $n_{i}^{(\alpha)}$ is the unit vector normal to the slip plane, and $s_{i}^{(\alpha)}$ is the unit vector in the slip direction.

The elastic response can be expressed in terms of a hypo-elastic law

$$
{\stackrel{\Delta}{\sigma^{*}}}_{i j}=L_{i j k l} D_{k l}^{*}
$$

where $L_{i j k l}$ is the fourth-rank elasticity tensor with Voigt symmetry and $\stackrel{\Delta^{*}}{\sigma_{i j}}$ is the Jaumann rate of Cauchy stress, $\sigma_{i j}$, co-rotational with the lattice spin.

The objective stress rate used here, is given by

$$
{\stackrel{\Delta}{\sigma^{*}}}_{i j}=\dot{\sigma}_{i j}-W_{i k}^{*} \sigma_{k j}-W_{k j}^{*} \sigma_{k i}
$$

where $\dot{\sigma}_{i j}$ is material time-derivative of Cauchy stress. 
The objective stress rate, $\stackrel{\Delta}{\sigma_{i j}}$, co-rotational to the material element can be derived as

$$
{\stackrel{\Delta}{\sigma_{i j}}}=L_{i j k l}\left(D_{k l}-D_{k l}^{P}\right)-W_{i k}^{P} \sigma_{k j}-W_{j k}^{P} \sigma_{k i}
$$

where $L_{i j k l}$ are the elastic moduli and for elastic isotropy can be expressed as

$$
L_{i j k l}=\mu\left(\delta_{i k} \delta_{j l}+\delta_{j k} \delta_{i l}\right)+\lambda \delta_{i j} \delta_{k l}
$$

where $\delta_{i j}$ is the Kronecker delta, $\lambda$ and $\mu$ are the Lame constants.

For a rate-dependent inelastic formulation, the slip-rates are functions of the resolved shear and reference stresses and a power law formulation by Hutchinson (1976)

$$
\dot{\gamma}^{(\alpha)}=\dot{\gamma}_{r e f}^{(\alpha)}\left[\frac{\tau^{(\alpha)}}{\tau_{r e f}^{(\alpha)}}\right]\left[\frac{\left|\tau^{(\alpha)}\right|}{\tau_{r e f}^{(\alpha)}}\right]^{\frac{1}{m}-1} \quad \text { no sum on } \alpha
$$

is used here. The reference shear strain rate, $\dot{\gamma}_{\text {ref }}^{(\alpha)}$, corresponds to a reference shear stress, $\tau_{r e f}^{(\alpha)}$. The exponent $m$ describes the material rate sensitivity and is given by

$$
m=\frac{\partial \ln \tau^{(\alpha)}}{\partial \ln \dot{\gamma}^{(\alpha)}}
$$

In other words, $m$ is the ratio of the rate of change of the resolved shear stress on each 
slip-system to the logarithmic rate of change of the slip rate on each slip-system. For shear slip-rates smaller than a critical value, the lattice motion is thermally activated. The rate sensitivity parameter is much less than one for values of the shear slip-rate smaller than a critical value (see, for example, Magnusen, Follansbee et al., 1984). The rate sensitivity parameter is approximately equal to one for slip rates greater than the critical slip rate, and this flow is characterized by drag controlled dislocation motion. The rate-independent limit is achieved as $m$ approaches zero. For multiple slip, $\gamma$, is taken as the sum of the accumulated plastic strains on all slip-systems, $n$,

$$
\gamma=\sum_{\alpha=1}^{n}\left|\gamma^{(\alpha)}\right|
$$

where $\tau^{(\alpha)}$ is the resolved shear stress and is given in terms of the Cauchy stress by

$$
\tau^{(\alpha)}=P_{i j}^{(\alpha)} \sigma_{i j}
$$

From the balance of energy, with no thermal conduction, the time rate of change of temperature is related to the rate of the plastic work by

$$
\dot{T}=\frac{\chi}{\rho c_{p}} \sigma_{i j}^{\prime} D_{i j}^{P},
$$

where $\chi$ is the fraction of the plastic work converted to heat, $\sigma_{i j}^{\prime}$ is the deviatoric stress, $\rho$ is the material density, and $c_{p}$ is the specific heat of the material. Due to the high strain rate 
deformation of the crystal, the rate of change of temperature is strictly a function of adiabatic heating.

\subsection{Evolutionary Equations for the Mobile and Immobile Dislocation-densities}

To gain a more fundamental understanding of dislocation motion, interaction, and transmission on material failure modes, the crystal plasticity constitutive formulations is coupled to internal variables that account for a local description of the dislocation structure in each crystal. Specifically, the mobile and the immobile dislocation-densities have been used as the internal variables in the present constitutive formulation. In inelastic deformations, the characteristics of the microstructure are governed by the mechanisms of dislocation production and dynamic recovery. As the material is strained, immobile dislocations are stored in each crystal, and these dislocations act as obstacles for evolving mobile dislocations. Therefore, the immobile and mobile dislocation-densities can be coupled, due to the continuous immobilization of mobile dislocations.

The reference stress, on each slip-system, can be given as a function of $\rho_{i m}^{(\alpha)}$, the immobile dislocation-density. The reference stress that is used here is a modification of widely used classical forms (see, for example, Mughrabi, 1987) that related the reference stress to a square root dependence on the dislocation-density as

$$
\tau_{r e f}^{(\alpha)}=\tau_{y}^{(\alpha)}+G b \sum_{\xi=1}^{12} a_{\xi} \sqrt{\rho_{i m}^{\xi}},
$$


where $G$ is the shear modulus, $b$ is the magnitude of the Burgers vector, $\tau_{y}^{(\alpha)}$ is the static yield stress, and the coefficients, $a_{\xi}(\xi=1,12)$ are interaction coefficients, and generally have a magnitude of unity.

Now consider a given state for a deformed material, which has a dislocation structure of total dislocation-density, $\rho^{(a)}$. This total dislocation-density is assumed to be additively decomposed, into a mobile dislocation-density, $\rho_{m}^{(\alpha)}$, and an immobile dislocation-density, $\rho_{i m}^{(\alpha)}$, as

$$
\rho^{(\alpha)}=\rho_{m}^{(\alpha)}+\rho_{i m}^{(\alpha)}
$$

Following the approach of (Gottstein and Argon, 1987), we have assumed that during an increment of strain, an immobile dislocation-density rate is generated, which will be denoted by $\dot{\rho}_{i m}^{(\alpha)+}$, and an immobile dislocation-density rate is annihilated, which will be denoted by $\dot{\rho}_{i m}^{(\alpha)-}$ on each slip-system as

$$
\frac{d \rho_{i m}^{(\alpha)}}{d t}=\dot{\rho}_{i m}^{(\alpha)+}+\dot{\rho}_{i m}^{(\alpha)-}
$$

It is assumed that $\dot{\rho}_{m}^{(\alpha)+}$ corresponds to a generation of mobile dislocation-densities, and $\dot{\rho}_{m}^{(\alpha)-}$ corresponds to an annihilation of mobile dislocation-densities as

$$
\frac{d \rho_{m}^{(\alpha)}}{d t}=\dot{\rho}_{m}^{(\alpha)+}+\dot{\rho}_{m}^{(\alpha)-}
$$


The balance between dislocation generation and annihilation, equations (2.21b-c), is the basis for the evolution of mobile and immobile dislocation-densities as a function of strain.

Plastic deformation of the crystal is assumed to begin with the easy glide stage, stage I. In this stage, most of the dislocations belong to the primary slip-system, and very little slip takes place on secondary slip-systems. Also, dislocation-densities are comparatively low, and the details of dislocation interaction and accumulation have been substantiated by TEM and other high-resolution methods. In the second stage of hardening (stage II), secondary slipsystems are activated, and dislocation clusters and cell walls begin to form. The third stage of hardening is characterized by the annihilation and the rearrangement of dislocations. For a detailed experimental overview of dislocation emission, interaction, trapping, and annihilation for the three hardening stages in FCC materials, see, for example, Mitchell (1964); Anongba, Bonneville et al. (1993); Anongba, Bonneville et al. (1993); and Argon and Haasen (1993).

In stage I, dipoles and multipoles are formed after mobile dislocations emitted from a source are trapped by dislocations of opposite signs on parallel slip planes. The back stress at the source is due to the dislocation emitted by the source. If the flow stress is greater than this back stress, the source will continue to emit dislocations, and dislocations that are trapped can break free. A large number of dipoles, multipoles, and loops are formed in easy glide by forest interactions, cross-slip around obstacles, and interactions between dislocations on parallel slip planes. Dipoles and multipoles occur in well-spaced clusters, thus allowing primary dislocations to glide over long distances. Using the equation (2.21c), this is given by 


$$
\frac{d \rho_{m}^{(\alpha)}}{d t}=\text { rate of generation }- \text { rate of trapping. }
$$

The rate of dislocation generation is proportional to the distance traveled by the emitted dislocations from a dislocation source with density $\rho_{\text {source }}^{(\alpha)}$. This distance, $y_{\text {back }}$, is related to the decrease of the back stress on the dislocation-density source, $\rho_{\text {source }}^{(\alpha)}$, after previously emitted dislocations have traveled this distance $y_{\text {back }}$. Hence, equation (2.22) can be written as

$$
\frac{d \rho_{m}^{(\alpha)}}{d t}=\rho_{\text {source }}^{(\alpha)} \frac{\bar{v}}{y_{\text {back }}}
$$

where $\bar{v}$ is mobile dislocation average velocity. Using Orowan's equation,

$$
\dot{\gamma}^{(\alpha)}=\rho_{m}^{(\alpha)} b \bar{v},
$$

and equation (2.23), we obtain the following equations:

$$
\frac{d \rho_{m}^{(\alpha)}}{d \gamma}=\frac{g_{\text {source }}}{b^{2}}\left(\frac{\rho_{i m}^{(\alpha)}}{\rho_{m}^{(\alpha)}}\right),
$$

where $b$ is the modulus of the Burgers vector, $g_{\text {source }}$ is a coefficient pertaining to an increase in the mobile dislocation-density due to dislocation sources. 
In stage II, dipole clusters multiply and join together, so that primary glide dislocations are efficiently blocked. Hardening increases in this stage are due to an increase in dislocation tangles. The forest dislocations of these systems serve as obstacles for the primary dislocations. The mobile dislocations are immobilized with a mean free path proportional to $\left(\rho_{i m}^{(\alpha)}\right)^{1 / 2}$. Spatially organized forest structures and tangles such as Frank nets, cell walls, or sub-boundaries can act as immobilization sites at this stage of the deformation.

Also thermally activated cross slip can block the glide dislocations. Since the rate of trapping is related to an increase in rate of growth of immobile dislocations, using equation (2.22), the coupled mobile and immobile dislocation-density evolution equations for this stage are given by

$$
\begin{aligned}
& \frac{d \rho_{m}^{(\alpha)}}{d \gamma}=-\frac{g_{\text {immob }}}{b} \sqrt{\rho_{i m}^{(\alpha)}}-\frac{g_{\text {minter }}}{b^{2}} \exp \left(-\frac{H}{\kappa T}\right), \\
& \frac{d \rho_{i m}^{(\alpha)}}{d \gamma}=\frac{g_{\text {immob }}}{b} \sqrt{\rho_{\text {im }}^{(\alpha)}}+\frac{g_{\text {minter }}}{b^{2}} \exp \left(-\frac{H}{\kappa T}\right),
\end{aligned}
$$

where $b$ is the modulus of the Burgers vector, $g_{\text {immob }}$ is a coefficient related to the immobilization of mobile dislocations, and $g_{\text {minter }}$ is a coefficient related to the trapping of mobile dislocations due to thermally activated cross-slip.

At moderate and large strains, dynamic recovery characterizes the stage III. At temperatures lower than $40 \%$ of the melting temperature, the main mechanism of recovery is annihilation of the screw segments of opposite signs on the expanding dislocation loops. The evolution for the immobile dislocation-density for this stage is 


$$
\frac{d \rho_{i m}^{(\alpha)}}{d \gamma}=-g_{\text {recov }} \exp \left(-\frac{H}{\kappa T}\right) \rho_{i m}^{(\alpha)},
$$

where $g_{\text {recov }}$ is a coefficient related to the rearrangement and annihilation of immobile dislocations.

Equations (2.25-2.28) can be combined to obtain a coupled set of nonlinear evolutionary equations

$$
\begin{aligned}
& \frac{d \rho_{m}^{(\alpha)}}{d t}=\dot{\gamma}^{(\alpha)}\left[\frac{g_{\text {sour }}}{b^{2}}\left(\frac{\rho_{\text {im }}^{(\alpha)}}{\rho_{m}^{(\alpha)}}\right)-\frac{g_{\text {minter }}}{b^{2}} \exp \left(-\frac{H}{\kappa T}\right)-\frac{g_{\text {immob }}}{b} \sqrt{\rho_{\text {im }}^{(\alpha)}}\right], \\
& \frac{d \rho_{i m}^{(\alpha)}}{d t}=\dot{\gamma}^{(\alpha)}\left[\frac{g_{\text {minter }}}{b^{2}} \exp \left(-\frac{H}{\kappa T}\right)+\frac{g_{\text {immob }}}{b} \sqrt{\rho_{\text {im }}^{(\alpha)}}-g_{\text {re cov }} \exp \left(-\frac{H}{\kappa T}\right) \rho_{\text {im }}^{(\alpha)}\right],
\end{aligned}
$$

where $g_{\text {sour }}$ is a coefficient pertaining to an increase in the mobile dislocation-density due to dislocation sources, $g_{\text {minter }}$ is a coefficient related to the trapping of mobile dislocations due to forest intersections, cross-slip around obstacles, or dislocation interactions, $g_{\text {recov }}$ is a coefficient related to the rearrangement and annihilation of immobile dislocations, $g_{\text {immob }}$ is a coefficient related to the immobilization of mobile dislocations, $\mathrm{H}$ is the activation enthalpy, and $\kappa$ is Boltzmann's constant. As these evolutionary equations indicate, the dislocation activities related to recovery and trapping are coupled to thermal activation. The thermal activation energy temperature is updated as a function of the energy given by (2.19). 


\subsection{Determination of the Coefficients for the Coupled Evolutionary Equations}

To couple the evolutionary equations to the crystal plasticity formulations, the four $g$ coefficients in (2.29-2.30), and the enthalpy, $\mathrm{H}$, must be determined as functions of the deformation mode. The enthalpy, $\mathrm{H}$, is determined by defining an exponential ratio of the current temperature to the reference temperature, following Paidar, Pope et al. (1984), a form of activation enthalpy for cross-slip which accounts the cross-slip effects. The four $g$ coefficients are determined using two general conditions, pertaining to the evolution of dislocation-densities in crystalline materials, have been used:

(i) that the mobile and immobile dislocation-densities saturate at large strains;

(ii) that the relaxation of the mobile dislocation-density to a quasi-steady state value occurs much faster than the variation of the immobile dislocation-density.

The conditions are invoked based on the arguments by Mecking and Kocks (1981), Walgraef and Aifantis (1985); Walgraef and Aifantis (1985); Walgraef and Aifantis (1985); Walgraef and Aifantis (1985), and Estrin and Kubin (1988); and Kubin and Estrin (1988). They used similar arguments to determine coefficients in equations pertaining to the evolution of mobile and immobile dislocation-densities. The saturation of both the immobile and mobile dislocation-densities at large strains and the their to different quasi-steady values in FCC materials have been experimentally substantiated by several investigators (see, for example, Mughrabi, 1987; Hansen, 1990; Bay, 1992). For a detailed presentation, see to Zikry and Kao (1996); Zikry and Kao (1996); and Zikry and Kameda (1998). 


\section{References}

Anongba, P. N. B., J. Bonneville, et al. (1993). "Hardening Stages of 112 Copper SingleCrystals at Intermediate and High-Temperatures .1. Mechanical-Behavior." Acta Metallurgica Et Materialia 41(10): 2897-2906.

Anongba, P. N. B., J. Bonneville, et al. (1993). "Hardening Stages of 112 Copper SingleCrystals at Intermediate and High-Temperatures .2. Slip-systems and Microstructures." Acta Metallurgica Et Materialia 41(10): 2907-2922.

Argon, A. S. and P. Haasen (1993). "A New Mechanism of Work-Hardening in the Late Stages of Large-Strain Plastic-Flow in Fcc and Diamond Cubic-Crystals." Acta Metallurgica Et Materialia 41(11): 3289-3306.

Bay, B., Hansen, N., Hughes, D.A., and Kuhlmannwilsdorf, D. (1992). "Overview NO-96 Evolution of FCC deformation Structures in Polyslip." Acta Metallurgica Et Materialia 40(2): 205-219.

Estrin, Y. and L. P. Kubin (1988). "Plastic Instabilities - Classification and PhysicalMechanisms." Res Mechanica 23(2-3): 197-221.

Gottstein, G. and A. S. Argon (1987). "Dislocation Theory of Steady-State Deformation and Its Approach in Creep and Dynamic Tests." Acta Metallurgica 35(6): 1261-1271.

Hansen, N. (1990). "Cold deformation microstructures." Materials Science and Technology 6(11): 1039-1047.

Hutchinson, J. W. (1976). "Bounds and Self-Consistent Estimates for Creep of Polycrystalline Materials." Proceedings of the Royal Society of London Series aMathematical Physical and Engineering Sciences 348(1652): 101-127.

Kameda, T. and M. A. Zikry (1998). "Intergranular and transgranular crack growth at triple junction boundaries in ordered intermetallics." International Journal of Plasticity 14(8): 689-702.

Kubin, L. P. and Y. Estrin (1988). "Strain Nonuniformities and Plastic Instabilities." Revue De Physique Appliquee 23(4): 573-583.

Magnusen, P. E., P. S. Follansbee, et al. (1984). "The Effect of Strain Rate on the Deformation and Fracture-Behavior of Ductile Metals Containing Porosity." Journal of Metals 36(7): 54-54. 
Mecking, H. and U. F. Kocks (1981). "Kinetics of Flow and Strain-Hardening." Acta Metallurgica 29(11): 1865-1875.

Mitchell, W. I. (1964). "The Precipitation Behaviour of a Complex Nickel-Base Alloy." Zeitschrift Fur Metallkunde 55(10): 613-616.

Mughrabi, H. (1987). "A 2-Parameter Description of Heterogeneous Dislocation Distributions in Deformed Metal Crystals." Materials Science and Engineering 85(12): $15-31$.

Paidar, V., D. P. Pope, et al. (1984). "A Theory of the Anomalous Yield Behavior in L12 Ordered Alloys." Acta Metallurgica 32(3): 435-448.

Walgraef, D. and E. C. Aifantis (1985). "Dislocation Patterning in Fatigued Metals as a Result of Dynamical Instabilities." Journal of Applied Physics 58(2): 688-691.

Walgraef, D. and E. C. Aifantis (1985). "On the Formation and Stability of Dislocation Patterns .1. One-Dimensional Considerations." International Journal of Engineering Science 23(12): 1351-1358.

Walgraef, D. and E. C. Aifantis (1985). "On the Formation and Stability of Dislocation Patterns .2. Two-Dimensional Considerations." International Journal of Engineering Science 23(12): 1359-1364.

Walgraef, D. and E. C. Aifantis (1985). "On the Formation and Stability of Dislocation Patterns .3. 3-Dimensional Considerations." International Journal of Engineering Science 23(12): 1365-1372.

Zikry, M. A. and T. Kameda (1998). "Inelastic three dimensional high strain-rate dislocationdensity based analysis of grain-boundary effects and failure modes in ordered intermetallics." Mechanics of Materials 28(1-4): 93-102.

Zikry, M. A. and M. Kao (1996). "Dislocation based multiple-slip crystalline constitutive formulation for finite-strain plasticity." Scripta Materialia 34(7): 1115-1121.

Zikry, M. A. and M. Kao (1996). "Inelastic microstructural failure mechanisms in crystalline materials with high angle grain boundaries." Journal of the Mechanics and Physics of Solids 44(11): 1765-1798. 


\section{CHAPTER 3}

\section{NUMERICAL METHOD}

To update the stress state of the crystalline material, both the total deformation rate tensor, $D_{i j}$, and the plastic deformation rate tensor,$D_{i j}^{P}$, are needed. A brief outline of the numerical method will be presented; for further details see Zikry (1994). An implicit finite element method analysis has been used to obtain the total deformation rate tensor, $D_{i j}$. The displacements have been obtained by the quasi-Newton solution of the static-equilibrium equation, with BFGS iteration to ensure convergence of the quasi-Newton method. Once the displacements are obtained, the deformation tensor can be calculated. To overcome numerical problems associated with incompressible displacements, the $\bar{B}$ method has been used in the calculation of the deformation tensor. In the $\bar{B}$ method, the deformation gradient is decomposed into volumetric and deviatoric parts. The volumetric part of the deformation tensor is then computed at reduced quadrature points. The resulting volumetric deformation field eliminates spurious modes that can arise due to incompressible deformations. Once the deformation tensor is obtained from the updated nodal displacement values, the total deformation rate tensor, $D_{i j}$, and the total spin tensor,$W_{i j}$, can be calculated at each load level.

To solve for the plastic deformation rate tensor, $D_{i j}^{P}$, the time derivative of the resolved shear stress

$$
\dot{\boldsymbol{\tau}}^{(\alpha)}=\frac{d}{d t}\left(P_{i j}^{(\alpha)} \sigma_{i j}\right),
$$


is used together with the objective stress rate, and the assumption that the elastic modulus tensor is isotropic, to obtain the following system of nonlinear differential equations for each active slip-system $\alpha$ :

$$
\dot{\boldsymbol{\tau}}^{(\alpha)}=L_{i j k l} P_{i j}^{(\alpha)} D_{k l}^{*}
$$

in expanding form

$$
\dot{\boldsymbol{\tau}}^{(\alpha)}=2 \mu P_{i j}^{(\alpha)}\left[D_{i j}-\sum_{\xi=1}^{12} P_{i j}^{(\xi)} \dot{\gamma}_{r e f}^{(\xi)}\left(\frac{\tau^{(\xi)}}{\tau_{r e f}^{(\xi)}}\right)^{\frac{1}{m}}\right] .
$$

It has also been assumed, in the derivation of equation (3.3), that the lattice spin is a function of the elastic spin in all three directions

$$
\begin{aligned}
& \dot{s}_{i}^{(\alpha)}=W_{i j}^{*} s_{j}^{(\alpha)}, \\
& \dot{n}_{i}^{(\alpha)}=W_{i j}^{*} n_{j}^{(\alpha)},
\end{aligned}
$$

where the elastic lattice spin is obtained as

$$
W_{i j}^{*}=W_{i j}-W_{i j}^{P} .
$$


The solution to the system of ordinary differential equation (3.3) is numerically difficult, not only due to the nonlinearity of the resolved shear stress, but also because the system of equation is numerically stiff in certain time intervals. The different time scales pertaining to the resolved shear stress on each slip-system cause the numerical stiffness. These results in eigenvalues correspond to the Jacobian of the initial value problem that are widely varying. This leads to the growth of numerically propagated error, i.e., instability in the solution of the system of differential equations. The computational scheme developed by Zikry (1994) is used to solve the system of equations (3.3). This algorithm is also used to update the evolutionary equations for the immobile and mobile dislocation-densities.

Since the system of equations given by equation (3.3) is only stiff in some regions of the integration domain, an explicit fifth-order accurate Runge-Kutta method is used over most of the time domain. The propagated error is measured by the growth in the local truncation error. If the time-step must be restricted due to stability and not accuracy, a backward Euler method is used. The backward Euler method is both A-stable and stiffly stable; it is also an order one Backward Differentiation Formula (BDF). The algorithm methodology is as follows:

Automatic step control has been achieved by using step doubling on the RungeKutta fourth-order method. Two approximate solutions are taken, one solution of step size $2 \mathrm{~h}$ and a second solution with two steps, each of size h. Since the original method is fourthorder, the two numerical methods are related by

$$
\tau(t+2 h)=\hat{\tau}_{1}+(2 h)^{5} \phi+O\left(h^{6}\right)+\cdots,
$$




$$
\tau(t+2 h)=\hat{\tau}_{2}+2(h)^{5} \phi+O\left(h^{6}\right)+\cdots,
$$

where $\phi$ is of the order $\tau^{5}(t) / 5$ !. Furthermore, the two numerical methods are combined to give a solution of fifth-order accuracy

$$
\tau(t+2 h)=\hat{\tau}_{2}+\frac{\Delta}{15}+O\left(h^{6}\right)+\cdots
$$

where $\Delta$ is the local truncation error which measures how well the solution is approximated at each time step. Based on this error measurement, an adjusted time step is calculated

$$
h_{\text {new }}=F h_{\text {old }}\left|\frac{\Delta_{0}}{\Delta_{1}}\right|^{0.20},
$$

where $h_{\text {new }}$ is the adjusted time step, and $h_{\text {old }}$ is the initial time step. The actual accuracy, $\Delta_{1}$, is measured by the supremum norm as max $\left|\tau_{1}-\tau_{2}\right|$, and $\Delta_{0}$ is the desired accuracy measured by $\varepsilon H$. Here $\varepsilon$ is the tolerance level supplied by the user and $H$ is a scaling factor for fractional errors for the $i^{t h}$ equation given by $|\tau|+\left|h \frac{d \tau}{d t}\right|$, where $h$ is the initial time step. The factor $F$ serves to keep the new time step small enough to be accepted if the truncation error in the next time step is growing. Based on equation (3.9), the time step is increased if the truncation error is smaller than the desired accuracy, and conversely the time step is decreased if the truncation error is greater than the desired accuracy.

Since Runge-Kutta methods have finite stability regions, there can be a growth in the 
propagated error and, therefore, the time step in certain time domains, can be restricted due to stability and not accuracy requirement by $\varepsilon H$. This is an indication of stiff behavior. In the present algorithm, the largest allowable time step is chosen, i.e., the time step on the stability boundary. This implies that the local errors are of the same magnitude as the accuracy tolerance used in equation (3.9). If the time step is unduly restricted due to stability, the solution will proceed in time, albeit inefficiently, due to the necessity of using intolerably small time steps. To correctly identify the regions of numerical stiffness, and to distinguish a step reduction due to accuracy from a time step reduction due to stability, a stiffness ratio, $S_{R}$, has been defined as

$$
S_{R}=\frac{|\operatorname{Re} \lambda|_{\max }}{|\operatorname{Re} \lambda|_{\min }}\left(\frac{1}{t_{2}-t_{1}}\right),
$$

where $|\operatorname{Re} \lambda|_{\max }$ and $|\operatorname{Re} \lambda|_{\min }$ are the greatest and smallest absolute values of the real parts of the eigenvalues of the Jacobian of the system of ordinary differential equations given by equation (3.3), and $t_{2}-t_{1}$ is the time interval of the integration. A large stiffness ratio, $S_{R}$, indicates that the ratios of the eigenvalues are dispersed relative to the time scale.

When the time step is restricted due to the presence of these widely varying eigenvalues, this is a stability problem, and an indication that the initial value problem is numerically stiff. An increasing stiffness ratio is an indication that for a specified deformation mode, the slip rate, $\dot{\gamma}^{(\alpha)}$, are much greater for one slip-system than for the other active slip-systems; namely, one of the slip-systems may be dominating the deformation process. The domination of one slip-system over other active slip-systems can occur, for 
example, when a macroscopic shear ban is forming in a crystalline solid (see, for example, Zikry, 1994).

If the stiffness ratio is increasing, then this is an indication of numerical stiffness, since the time step is being reduced due to stability considerations. In the present analysis, when stiff behavior is encountered, the integration is automatically switched to the backward Euler scheme. The quasi-Newton method has been used to solve the system of nonlinear algebraic equations.

The previous detailed algorithm, used to update the plastic deformation rate tensor, has also been used to update the coupled nonlinear mobile and immobile dislocation-density evolutionary equations (2.29-2.30). 


\section{Reference}

Zikry, M. A. (1994). "An Accurate and Stable Algorithm for High Strain-Rate Finite Strain Plasticity." Computers \& Structures 50(3): 337-350. 


\section{CHAPTER 4}

\section{GRAIN-BOUNDARY INTERFACIAL REGIONS AND INTERACTION MECHANISMS}

\subsection{Introduction}

It is well understood from numerous investigations pertaining to the experimental characterization of GB physical systems, such as intrinsic thermo-mechanical properties, operative crystallographic systems, and dislocation mechanisms that these all may be substantially different than those associated with the aggregate crystalline bulk response (see, for example, Randle, 1997; Dingley and Pond, 1979; Liu and Baker, 1993). These studies further indicate that GB regions normally have inherently unique geometrical structures due to misorientations between adjacent grains that occur over distinct spatial widths and distributions. These GB interfacial regions can strongly affect and control the thermomechanical response and ductile failure of polycrystalline materials, and hence a detailed understanding of these interrelated mechanisms has to be obtained for accurate predictions of failure initiation and evolution in crystalline aggregate systems. These cited SEM, TEM, and X-ray characterization studies, can provide valuable understanding of what is happening at a specific deformation level, but they do not generally provide a detailed understanding of how failure initiates and evolves, and what the dominant mechanisms associated with failure are.

As noted by Randle (1997), GB regions can be envisioned as highly localized transitional regions of finite widths with an atomic periodicity that is different than that of the contacting crystals. (Figure 4.1) This transitional region can consist of defects, such as 
different dislocations, serrations, microfacets, and protrusions. Furthermore, at homologous temperatures (approximately at $40 \%$ of the melting temperature), GB regions can be strong obstacles to dislocation motion, and this can result in substantial increases in the overall flow response of polycrystalline aggregates in comparison with the response of monocrystalline structures. In addition, interfacial misorientations between grains can involve the translation and rotation of one crystalline lattice with respect to adjacent grains.

In this study, the orientation of each crystal is given by three Euler angles, $\varphi_{1}, \Phi, \varphi_{2}$, and the orientation of the GB is given by a normal vector to the GB plane, $\tilde{n}_{g b}$. Therefore, these four degrees of freedom will be sufficiently representative for planar deformation.

\subsection{Euler Space}

In this section, we will introduce a model, which will be used with the dislocationdensity based multiple-slip crystalline formulation and the specialized computational schemes, that accounts for interfacial effects and interaction effects. It will be shown that these interfacial regions have a dominant role in failure initiation and evolution. Kinematical conditions will be introduced that account for dislocation-density and slip-rate transmission and blockage in GB regions. As noted earlier, current crystal plasticity formulations do not account for GB mechanisms and their effects on aggregate response and failure by nanoindentation. Most of these formulations assume that the strains are compatible and that stress equilibrium is preserved at GB interfaces.

In the Bunge system, Randle (1993); Randle (1993); Randle (1993); Randle (1993),

the global crystallographic axes $([100],[010],[001])$ are designated as $\left(x_{2}, y_{2}, z_{2}\right)$, and the 
local rotated axes are designated $\left(x_{1}, y_{1}, z_{1}\right)$. Three successive rotations, based on specified Euler angles, are needed to represent the crystallographic orientation of each grain. The three rotations are as follows:

Rotation $m_{1}$ : rotate about $z_{1}$ by $\varphi_{1}$, so that the rotated $x_{1}^{\prime}$ lies in the plane $\left(x_{1}, x_{2}\right)$. The rotated system now is $\left(x_{1}^{\prime}, y_{1}^{\prime}, z_{1}^{\prime}\right)$.

Rotation $m_{2}$ : rotate about $x_{1}^{\prime}$ by $\Phi$, so that the rotated $z_{1}^{\prime \prime}$ is parallel to $z_{2}$. The rotated system now is $\left(x_{1}^{\prime \prime}, y_{1}^{\prime \prime}, z_{1}^{\prime \prime}\right)$.

Rotation $m_{3}$ : rotate about $z_{1}^{\prime \prime}=z_{2}$ by $\varphi_{2}$, so that the rotated $x_{1}^{\prime \prime \prime}$ and $y_{1}^{\prime \prime \prime}$ coincide with $x_{2}$ and $y_{2}$, respectively.

The mathematical representations of Euler rotations are:

$$
\begin{aligned}
& \tilde{m}_{1}=\left[\begin{array}{ccc}
\cos \varphi_{1} & \sin \varphi_{1} & 0 \\
-\sin \varphi_{1} & \cos \varphi_{1} & 0 \\
0 & 0 & 1
\end{array}\right], \\
& \tilde{m}_{2}=\left[\begin{array}{ccc}
1 & 0 & 0 \\
0 & \cos \Phi & \sin \Phi \\
0 & -\sin \Phi & \cos \Phi
\end{array}\right], \\
& \tilde{m}_{3}=\left[\begin{array}{ccc}
\cos \varphi_{2} & \sin \varphi_{2} & 0 \\
-\sin \varphi_{2} & \cos \varphi_{2} & 0 \\
0 & 0 & 1
\end{array}\right] .
\end{aligned}
$$

For two adjacent grains, misorienting one with respect to the other is performed by rotating each crystal coordinate system by a transformation matrix, based on each crystal's Euler angles, with respect to a global polycrystal frame-of-reference. The misorientationtransformation matrix for Euler angles $\left(\varphi_{1}, \Phi, \varphi_{2}\right)$, is calculated by matrix multiplication of 
the rotation matrices $\left(\tilde{m}_{1}, \tilde{m}_{2}, \tilde{m}_{3}\right)$

$$
\tilde{M}=[M]=\tilde{m}_{3} \cdot \tilde{m}_{2} \cdot \tilde{m}_{1}
$$

Using equations (4.1) together with equation (4.2) will yield

$$
\tilde{M}=\left[\begin{array}{ccc}
\cos \varphi_{1} \cos \varphi_{2}-\sin \varphi_{1} \sin \varphi_{2} \cos \Phi & \sin \varphi_{1} \cos \varphi_{2}+\cos \varphi_{1} \sin \varphi_{2} \cos \Phi & \sin \varphi_{2} \sin \Phi \\
-\cos \varphi_{1} \sin \varphi_{2}-\sin \varphi_{1} \cos \varphi_{2} \cos \Phi & -\sin \varphi_{1} \sin \varphi_{1}+\cos \varphi_{1} \cos \varphi_{2} \cos \Phi & \cos \varphi_{2} \sin \Phi \\
\sin \varphi_{1} \sin \Phi & -\cos \varphi_{1} \sin \Phi & \cos \Phi
\end{array}\right]
$$

which is the misorientation-transformation matrix expressed in terms of Euler angles.

Dislocation glide occurs in definite crystallographic planes and directions. The combination of a particular slip plane and a slip direction in that plane is a slip-system. Facecentered cubic crystals deform on the close-packed octahedral $\{111\}$ planes in the close-packed directions. There are four planes with three different slip directions, therefore there are total of twelve slip-systems for each crystal. Hence, the two vectors representing the orientation of a specific slip-system are the normal to the slip plane $\tilde{n}$, and the slip vector $\tilde{s}$. The following transformation law for first-order Cartesian tensors defines the rotational slipsystem in each crystal

$$
\begin{aligned}
& n_{i}^{\prime}=M_{i j} n_{j}, \\
& s_{i}^{\prime}=M_{i j} s_{j} .
\end{aligned}
$$

The slip normal to and the slip direction vectors are given in the Table 4.1. The slip normal 
and the slip direction are updated as function of the elastic spin tensor, $W_{i j}^{*}$ (Equation 3.4).

Table 4.1. Slip-systems of f.c.c. metals

\begin{tabular}{cc}
\hline Slip-system \# & Slip-system \\
\hline 1 & $(111)[01 \overline{1}]$ \\
2 & $(111)[\overline{1} 01]$ \\
3 & $(111)[1 \overline{1} 0]$ \\
4 & $(\overline{1} 11)[01 \overline{1}]$ \\
5 & $(\overline{1} 11)[011]$ \\
6 & $(\overline{1} 11)[\overline{1} \overline{1} 0]$ \\
7 & $(1 \overline{1})[011]$ \\
8 & $(1 \overline{1} 1)[10 \overline{1}]$ \\
9 & $(1 \overline{1} 1)[\overline{1} \overline{1} 0]$ \\
10 & $(11 \overline{1})[011]$ \\
11 & $(11 \overline{1})[\overline{1} 0 \overline{1}]$ \\
12 & $(11 \overline{1})[1 \overline{1} 0]$ \\
\hline
\end{tabular}

\subsection{Kinematics of GB and Dislocation-density Interaction}

There is a myriad of dislocation-density interactions with GB interfaces, such as:

- GB absorption of lattice dislocations without dissociation into GB-dislocations (GBD) (Baker and Gaydosh, 1987; Baker, Schulson et al., 1987; Liu and Baker, 1993);

- Partial dislocation transmission from one grain to the adjacent grain with a residual GBD left in the GB region (Lee, Robertson et al., 1992; Liu and Baker, 1993); 
- Full dislocation transmission from one grain to the adjacent grain with no residual GBD left in the GB region (Shen, Wagoner et al., 1988; Lee, Robertson et al., 1992; Liu and Baker, 1993);

- Dislocation absorption and subsequent re-emission from the GB (Shen, Wagoner et al., 1988);

We will focus on the following interactions:

- Full and partial dislocation-density transmission from one grain to neighboring grains;

- Full and partial dislocation-density transmission into GB and blockage at neighboring grains;

- Dislocation-density impedance and potential pile-ups.

These kinematical scenarios provide a general methodology that can be used as a framework for GB interfacial mechanism and interactions.

\subsection{GB Interaction Scheme}

Dislocation-density transmission and impedance from one grain to neighboring grains across GB regions, will be determined through the following interrelated steps:

- A potential dislocation-density envelope will be used to identify regions of high dislocation activities;

- Coordinate frame transformations will be performed with respect to the GB to identify the direction of the slip-system associated with critical dislocation-density 
systems;

- Conditions will be obtained for geometric compatibility to determine whether neighboring slip-systems are oriented for transmission or impedance through GB;

- If transmission is possible, through the GB, a transformation factor will be calculated.

\subsection{Potential Dislocation-density Envelope}

It is essential to determine how dislocation-density evolves near GB regions and what the maximum values and corresponding active slip-system directions and orientations are, such that slip-system impedance and transmission can be controlled and monitored. Slip impedance at GB can lead to pile-ups and stress-accumulation, if neighboring slip-systems within the GB and adjacent grains are not activated and oriented for slip transmission. Therefore, it is crucial to identify the critical regions of dislocation-density accumulation. A potential dislocation-density envelope is calculated as follows:

- The maximum dislocation-density on the corresponding slip-system is identified in the region adjacent to the GB. This region corresponds to the first row of element adjacent to the GB.

- This process is repeated row by row (element by element) until the half of the grain, $0.5 a_{g}$, is transversed.

- From this information, the envelope is constructed as shown in Figure 4.2. The first element is identified as the leading point of the envelope of potential dislocationdensity activity. From the adjacent row, three elements are chosen. This process is repeated until the mid-point of the grain is reached and the envelope is completed. 
From this process, which can entail monitoring hundreds of elements per grain, we obtain the distribution of the total dislocation-density as a function of the radial pile up length, $l_{p}$ (Figure 4.2). This radial distribution is not constrained to be of any form. But if it is a dislocation-density pile-up, it will be a decreasing function of radial length.

What this provides is a distribution of the total dislocation-density as a function of length as shown in Figure 4.2. This length, $l_{p}$, is a radial line that emanates from the GB to the middle of the grain. This radial line encompasses the dislocation-density envelope. This potential dislocation-density envelope can now be used to identify regions, in all four adjacent grains, of potential transmission or impedance, or blockage. Therefore dislocationdensity could transmit from a grain to GB to neighboring grain or from a grain to GB, and could pile-up in one grain or pile-ups in two grains that are adjacent same GB.

\subsection{Dislocation-density Orientation and GB Coordinate Frame Transformation}

To be able to determine if the dislocation-density is piling-up or moving away from GBs, the orientation and direction has to be determined.

The GB normal vector $\tilde{n}_{g b}$ and the in-plane vector $\quad \tilde{m}_{g b}$ will be used to determine dislocation-density directions and orientations. These coordinate transformations have to be performed for all twelve-slip-systems on all four GB sides for each grain. A schematic explanation is shown in Figure 4.3, and the procedure is outlined below (for every slipsystem $\alpha)$ :

- Define the GB normal and in-plane vectors $\left(\tilde{n}_{g b}, \tilde{m}_{g b}\right)$; these are orthonormal vectors; 
- Obtain the orientation of the slip-system $a$ in terms of the slip normal $\tilde{n}^{(\alpha)}$ and the slip $\operatorname{direction} \tilde{s}^{(\alpha)}$;

- Obtain the slip rate value and its sign;

- Perform the following check to ensure that slip rate is positive:

$$
\text { If } \operatorname{sgn}\left\{\dot{\gamma}^{(\alpha)}\right\}<0 \Rightarrow \dot{\gamma}^{(\alpha)}=-\dot{\gamma}^{(\alpha)} \text {, and } \tilde{s}^{(\alpha)}=-\tilde{s}^{(\alpha)} \text {. }
$$

- Now, perform an inner product vector operation to align the slip-system orientation, $\tilde{s}^{(\alpha)}$, with the GB-reference-frame,

a) Check the inner product sign. If it is negative, change the sign of both slip direction and the slip rate for the slip-system, hence the inner product must satisfy the condition $\operatorname{sgn}\left\{\tilde{s}^{(\alpha)} \cdot \tilde{n}_{g b}\right\}>0$,

$$
\text { If } \operatorname{sgn}\left\{\tilde{s}^{(\alpha)} \cdot \tilde{n}_{g b}\right\}<0 \Rightarrow \dot{\gamma}^{(\alpha)}=-\dot{\gamma}^{(\alpha)} \text {, and } \tilde{s}^{(\alpha)}=-\tilde{s}^{(\alpha)} \text {. }
$$

b) Take the inner product of the slip-system normal, $\tilde{n}^{(\alpha)}$, and GB in-plane vector, $\tilde{m}_{g b}$, and check the product sign. If it is negative, change the sign of both the slip normal and the slip rate for the present slip-system; i.e., the inner product must satisfy the condition $\operatorname{sgn}\left\{\tilde{n}^{(\alpha)} \cdot \tilde{m}_{g b}\right\}>0$,

$$
\text { If } \operatorname{sgn}\left\{\tilde{n}^{(\alpha)} \cdot \tilde{m}_{g b}\right\}<0 \Rightarrow \dot{\gamma}^{(\alpha)}=-\dot{\gamma}^{(\alpha)} \text {, and } \tilde{n}^{(\alpha)}=-\tilde{n}^{(\alpha)} \text {. }
$$

Another important issue is the determination of the sign of the dislocation-densities. This is needed to determine if the dislocation-densities are piling-up at GBs or transmitting through GBs. The sign convention for transmission and pile-ups of dislocation-densities the four GBs are shown in Figure 4.4. The power law, equation (2.17), is used to determine if neighboring slip-systems are active. 


\subsection{Slip-plane Geometric Compatibility}

Dislocation-density transmission or impedance across GB interfacial regions is dependent not only on whether there are adjacent active slip-systems, but whether these slipsystems are geometrically compatible, such that transmission is possible. Geometric compatibility will be defined by tow angles, $\theta$ and $\beta$ (Figure 4.5). It will be assumed that both angles do not exceed critical values, $\theta_{c}$ and $\beta_{c}$. If these values are exceeded, then transmission through the GB is not possible.

$\theta$ is the angle between two intersection lines (vectors, $\left.\tilde{l}_{1}, \tilde{l}_{2}\right)$ that represent the lines formed by the intersection of slip planes and the GB interfacial layers as shown in Figure 4.6. The angle can be determined from the following cross product operation,

$$
\begin{aligned}
& \tilde{l}_{1}=\tilde{n}_{1} \times \tilde{n}_{g b}, \\
& \tilde{l}_{2}=\tilde{n}_{2} \times \tilde{n}_{g b},
\end{aligned}
$$

where $\tilde{n}_{1}$ and $\tilde{n}_{2}$ are the two normals to the slip planes across the interfacial layer, and $\tilde{n}_{g b}$ is the GB normal. The angle from this operation is

$$
\cos \theta^{\prime}=\frac{\tilde{l}_{1} \cdot \tilde{l}_{2}}{\left|\tilde{l}_{1}\right|\left|\tilde{l}_{2}\right|},
$$

and $\theta$ is determined based on the following two conditions, 


$$
\theta=\left\{\begin{array}{lll}
\left|\theta^{\prime}\right| & \text { if } & \theta^{\prime}<\frac{\pi}{2} \\
\pi-\left|\theta^{\prime}\right| & \text { if } & \theta^{\prime}>\frac{\pi}{2}
\end{array},\right.
$$

Geometrically, it is clear from Figure 4.6 that slip transmission is a maximum when $\theta=0$. In this situation, full transmission occurs. However, as $\theta$ increases, dislocation-density transmission will be impeded as noted by Werner and Prantl (1990). Thus, slip movement is impeded markedly when $\theta$ grows. As also noted by Davis, Teghtsoo.E et al. (1966) slip planes intersecting the boundary should maintain an angle of $15^{\circ}$ or less. Therefore, the critical angle, $\theta_{c}$, is taken as

$$
\theta \leq \theta_{c}=15^{\circ}
$$

The second condition in ensuring geometric compatibility is to observe both slip plane orientations along the interfacial region with respect to an axis normal to a plane containing the GB normal and in-plane vectors, as shown in Figure 4.7. And angle $\beta$ for this geometric compatibility is given by

$$
\cos \beta=\tilde{n}_{1} \cdot \tilde{n}_{2}
$$

Full transmission (with respect to $\beta$-compatibility) of slip takes place when $\beta=0$. As a kink starts to form, for increasing value of $\beta$, dislocation-densities will be impeded. We will assume the following condition for $\beta$-compatibility, 
$\beta \leq \beta_{c}=35^{\circ}$.

In summary, dislocation-densities can transmit when both conditions of $\theta$ compatibility and $\beta$-compatibility are satisfied in conjunction with the other conditions outlined in section 5.4.

\subsection{Transmission Distribution Factor}

It is obvious that geometric compatibility affects to a larger degree dislocationdensity transmission. Previously, we mentioned that the most favorable configuration occurs when $\theta=0$ and $\beta=0$. This indicates complete transmission. Deviation from this configuration would represent a partial transmission through the interface. A transmission

factor $\zeta$, based on $\theta$ and $\beta$, will be defined as

$$
\varsigma=\cos \theta \cos \beta
$$

This will provide a measure of how much of the dislocation-density penetrates through the GB and into neighboring grains.

\subsection{Summary}

Implementing these procedures is quite tedious and complex since all the twelve-slipsystems have to be continually monitored on all four GB regions for each grain. In some 
cases, pile-ups can be relieved by slip-system rotations in neighboring grains, or pile-ups may be intensified as the deformation and failure evolve. In other cases dislocation-density transmission through the GB, may occur by partial transmission on different slip-systems into neighboring grains. All of these situations need to be delineated for accurate predictions of the effects of GB interfacial regions on failure. 


\section{References}

Baker, I. and D. J. Gaydosh (1987). "Dynamic Recrystallization and Grain-Boundary Migration in B2-Feal." Metallography 20(3): 347-357.

Baker, I., E. M. Schulson, et al. (1987). "Insitu Straining of Ni3al in a Transmission ElectronMicroscope." Acta Metallurgica 35(7): 1533-1541.

Davis, K. G., Teghtsoo.E, et al. (1966). "Slip Band Continuity across Grain-boundaries in Aluminum." Acta Metallurgica 14(12): 1677-\&.

Dingley, D. J. and R. C. Pond (1979). "Interaction of Crystal Dislocations with GrainBoundaries." Acta Metallurgica 27(4): 667-682.

Lee, T. C., I. M. Robertson, et al. (1992). "Interaction of Dislocations with Grain-Boundaries in Ni3al." Acta Metallurgica Et Materialia 40(10): 2569-2579.

Liu, F. and I. Baker (1993). "A Compression Jig for X-Ray Topography of Ice." Measurement Science \& Technology 4(3): 416-421.

Randle, V. (1993). "Application of Microtexture Measurements in the Sem to GrainBoundary Parameters." Textures and Microstructures 20(1-4): 231-242.

Randle, V. (1993). "Grain-Boundary Planes and Their Energies as Estimated by Dihedral Angle Measurements in Nickel." Microscopy Microanalysis Microstructures 4(4): 349-358.

Randle, V. (1993). "Microtexture Investigation of the Relationship between Strain and Anomalous Grain-Growth." Philosophical Magazine a-Physics of Condensed Matter Structure Defects and Mechanical Properties 67(6): 1301-1313.

Randle, V. (1993). "On the Significance of Grain Junction Topology and Crystallography in Polycrystals." Scripta Metallurgica Et Materialia 28(8): 889-893.

Randle, V. (1997). "Orientation modelling of two-dimensional textures." Modelling and Simulation in Materials Science and Engineering 5(2): 117-127.

Shen, Z., R. H. Wagoner, et al. (1988). "Dislocation and Grain-Boundary Interactions in Metals." Acta Metallurgica 36(12): 3231-3242.

Werner, E. and W. Prantl (1990). "Slip Transfer across Grain and Phase Boundaries." Acta Metallurgica Et Materialia 38(3): 533-537. 


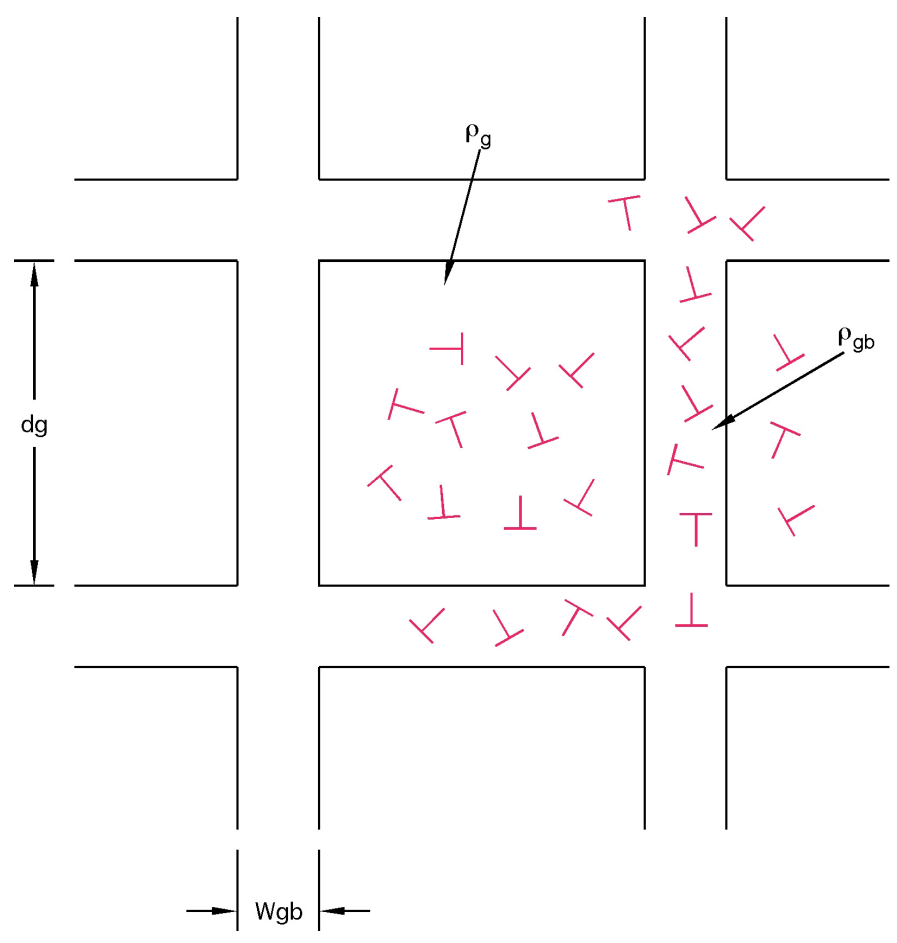

Figure 4.1. Grain and GB representation
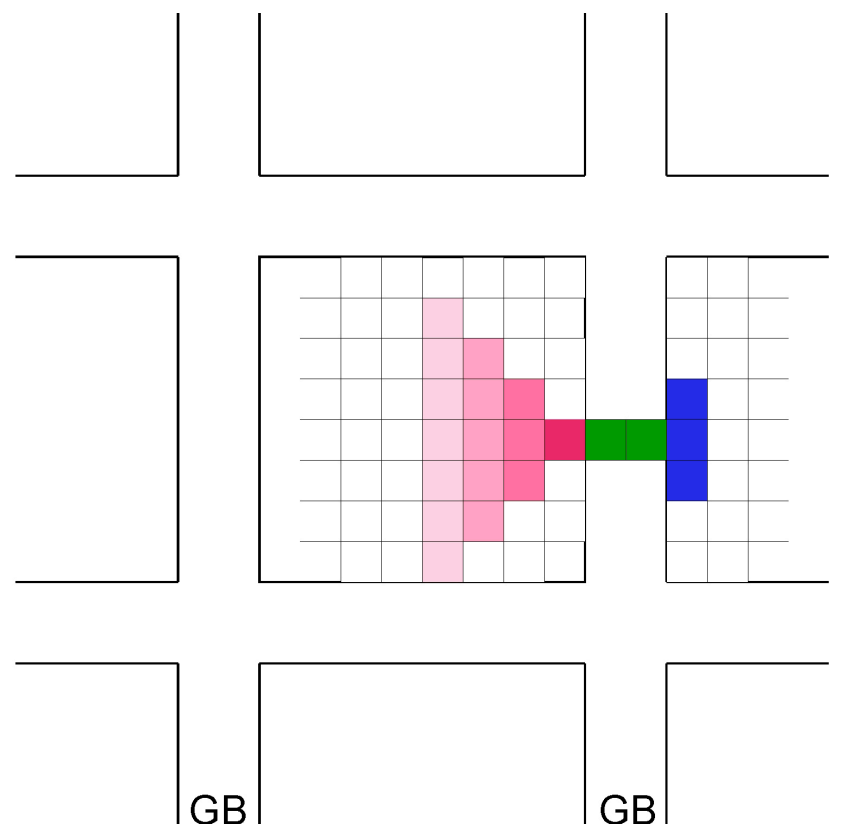

Leading Dislocation-density Element $\square$ Potential Dislocation-density Pile-up Envelope

GB Potential Transmission Zone $\quad$ Neighboring Grain Potential Transmission Zone

Figure 4.2. Dislocation-density envelops 


\section{Slip-rate Adjustment}

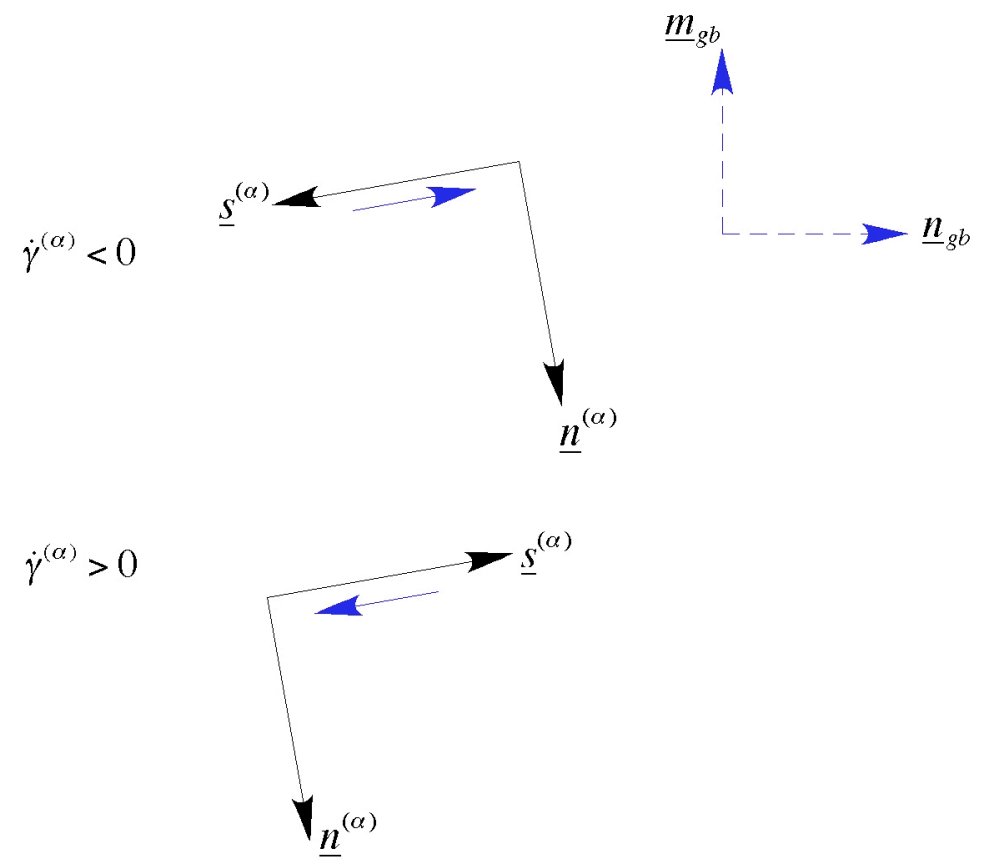

2. $\underline{s}^{(\alpha)} \cdot \underline{n}_{g b}>0$

$$
\begin{array}{rlr}
\mathrm{IF} \underline{s}^{(\alpha)} \cdot \underline{n}_{g b}<0 & \Rightarrow \quad \dot{\gamma}^{(\alpha)}>0 \\
\underline{s}^{(\alpha)} & =-\underline{s}^{(\alpha)} & \\
\dot{\gamma}^{(\alpha)} & =-\dot{\gamma}^{(\alpha)} &
\end{array}
$$

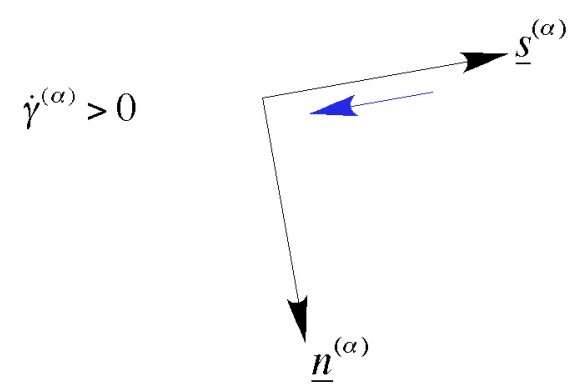

3. $\underline{n}^{(\alpha)} \cdot \underline{m}_{g b}>0$

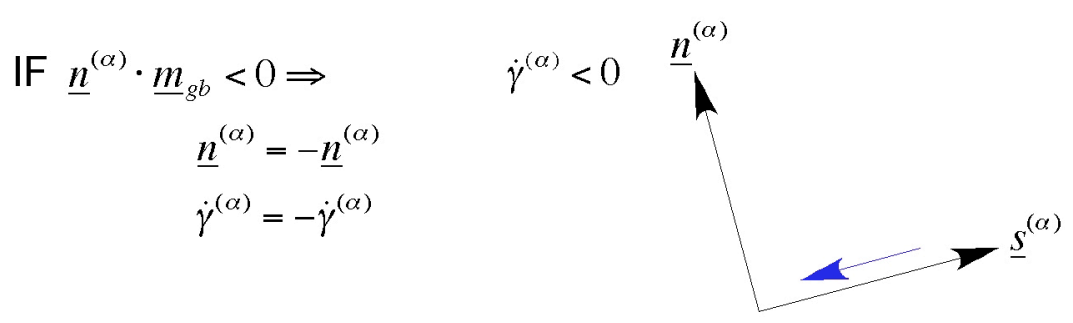

Figure 4.3. Schematic view for GB coordinates frame transformation 

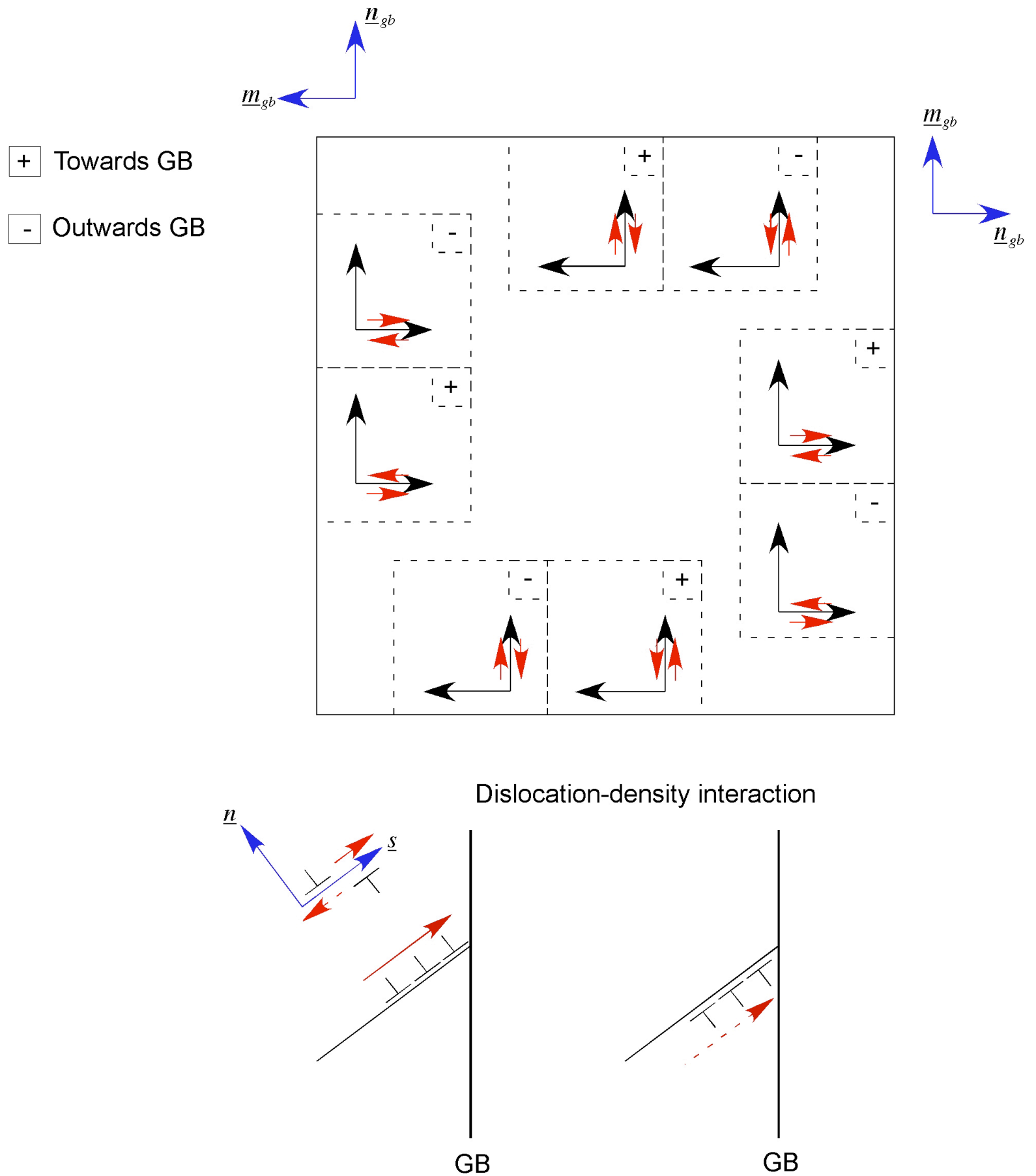

Figure 4.4. Dislocation-density sign convention along the four GBs 


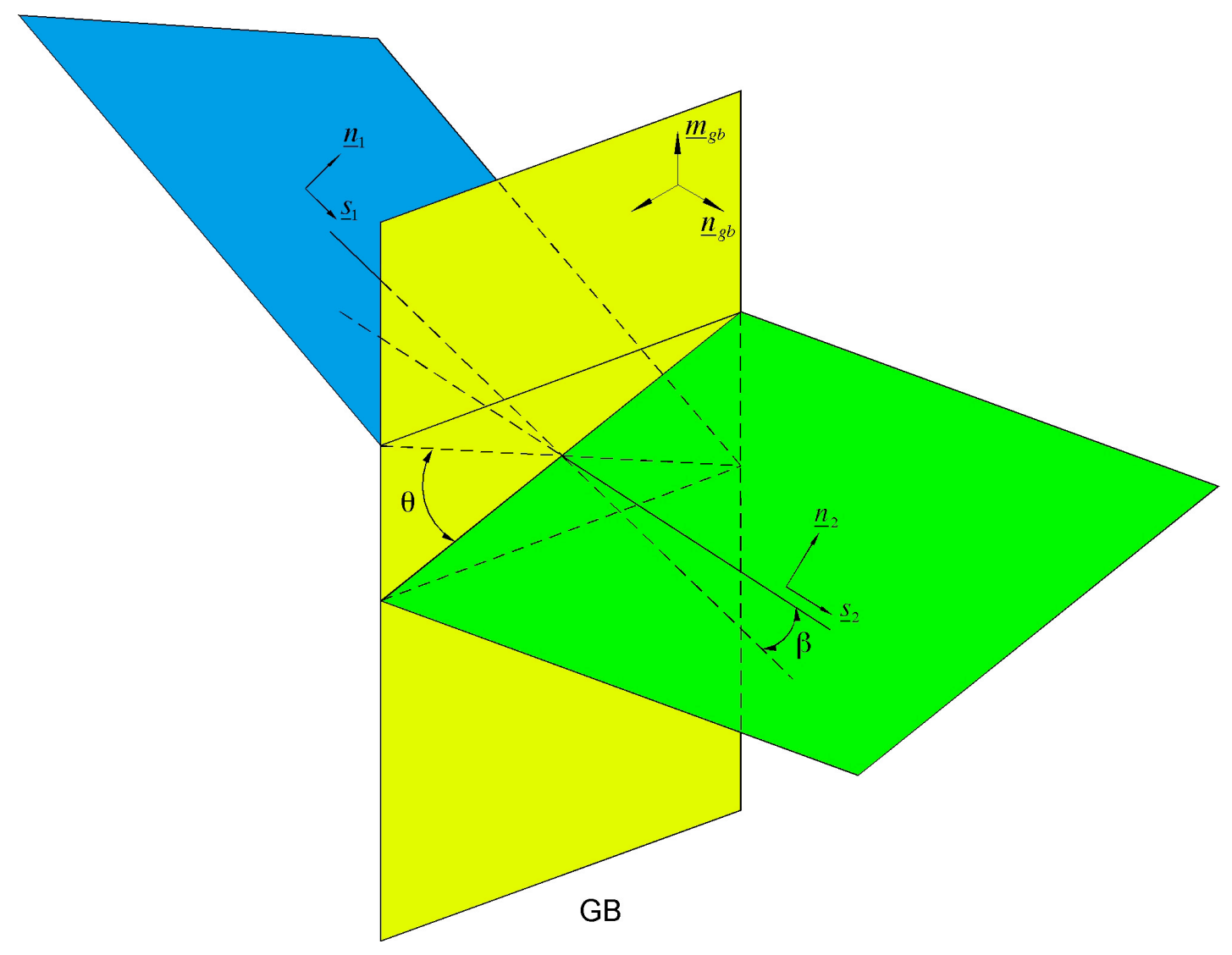

Figure 4.5. General slip-plane geometric compatibility

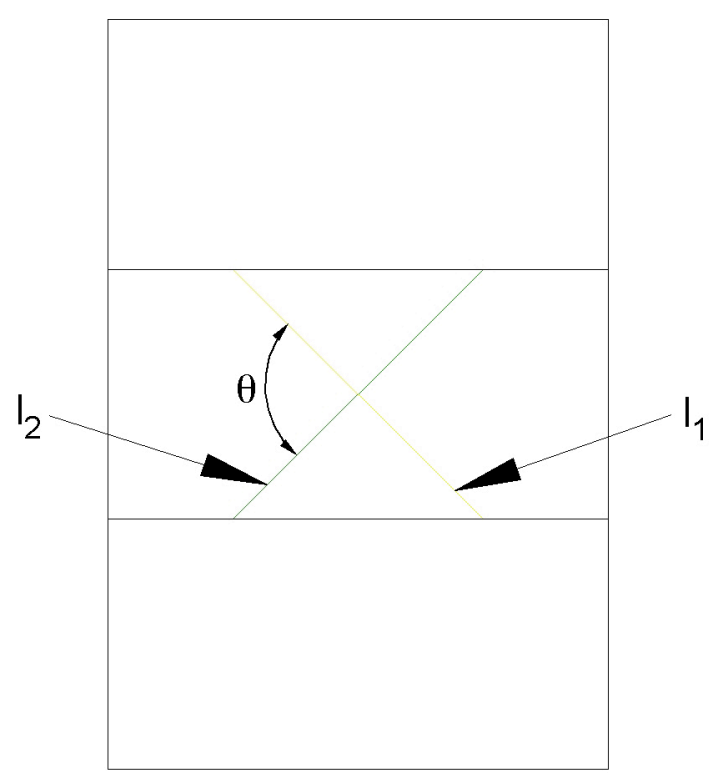

Figure 4.6. Slip-plane $\theta$-compatibility 


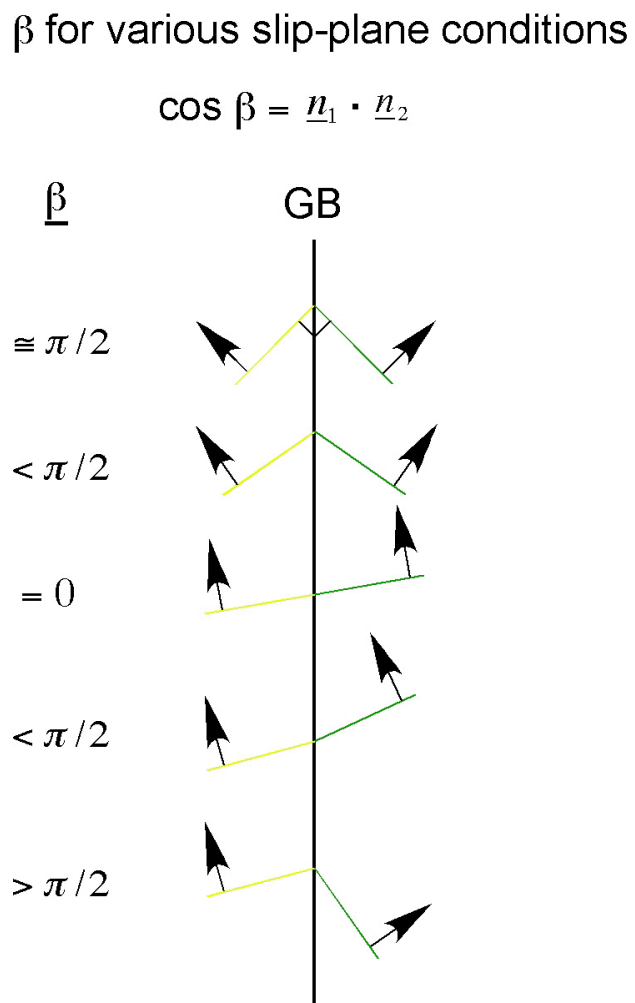

Figure 4.7. Slip-plane $\beta$-compatibility 


\section{CHAPTER 5}

\section{HIERARCHICAL MODELING OF NANOINDENTATION OF POLYCRYSTALLINE GOLD AGGREGATES}

\subsection{Hierarchical Modeling of Nanoindentation: Linking and Scaling of Molecular Dynamic Simulations with the Microstructurally Based Finite-Element Formulation}

In this section, the methodology for linking MD simulations with the microstructurally based FEM formulation is outlined. As indicated in Chapter 1, MD simulations are essential for predicting nucleation phenomena, such as dislocation formation and GB sliding. However, due to spatial and temporal limitations, MD modeling techniques cannot be used to understand and predict how the microstructure evolves beyond the nucleation stage, on length scales that would be commensurate with, for example, large inelastic deformations, dislocation-density evolution, GB effects, and lattice rotations in crystalline aggregates. Hence, the objective here is to use critical information pertaining to nanoindentation at the molecular level, and then scale it to the microstructural level to accurately predict and understand how different phenomena, such as dislocation-density transmissions and blockages and inelastic deformation modes evolve as a function of crystal structure, GB orientation and distribution, and grain orientation and size.

In this study, the proposed hierarchical approach is based on using displacement profiles from the MD simulations of nanoindentation to obtain scaling relations, which are related to the strains pertaining to indented depths. These strains, which are scale invariant, are then used in the microstructural FEM models by coarsening the grain-sizes. The accuracy of this scaling scheme is determined by comparing the FEM predicted hardness values with experimentally obtained measurements of gold crystalline aggregates. 


\subsubsection{Gold Crystalline Aggregate with Cube Orientation}

For the microstructural model, we used an aggregate with cube Euler angle orientations of $(0,0,0)$ for all the grains and GBs. This was done to simulate an aggregate with no GB effects, and hence to approach the limiting case of a single crystal (see, for example, Hasnaoui, Derlet et al., 2004). To illustrate how we linked the FEM formulation with the MD simulations, we analyzed two different sized gold crystal aggregates. A specimen with dimensions of $0.00975 \mathrm{~mm}$ in the vertical direction and $0.01635 \mathrm{~mm}$ in the horizontal direction, a grain-size of $0.003 \mathrm{~mm}$ and an aggregate 15 grains (Model 1), and a specimen with dimensions of $0.00957 \mathrm{~mm}$ in vertical direction and $0.01683 \mathrm{~mm}$ in the horizontal direction with a smaller grain-size of $0.0022 \mathrm{~mm}$ and a larger aggregate of 28 grains (Model 2). The material properties that were used in these models, which are representative of pure gold (McCann, 2004 and Pamula et al., 2001) are given in Table 5.1. It was assumed that all twelve slip-systems for this f.c.c. aggregates were potentially active. Using the method outlined in Kameda and Zikry (1998) (reference in chapter 2), the saturated immobile dislocation-density, $\bar{\rho}_{i m s}$, was calculated as $1.0 \times 10^{16} \mathrm{~m}^{-2}$ and the saturated mobile dislocation-density, $\bar{\rho}_{m s}$, was calculated as $4.3 \quad \times 10^{13} \mathrm{~m}^{-2}$. The initial mobile

dislocation-density of the grain bulk was chosen as $10^{7} \mathrm{~m}^{-2}$, and the initial immobile dislocation-density was chosen as $10^{10} \mathrm{~m}^{-2}$. These are values that are typical of f.c.c. crystalline aggregates. Using these values, the coefficient values and the enthalpy, needed for the evolution of the immobile and mobile dislocation-density equations (2.29-2.30), are calculated as

$$
\mathrm{g}_{\text {miniter }}=2.013, \mathrm{~g}_{\text {recov }}=4, \mathrm{~g}_{\text {immob }}=0.02, \mathrm{~g}_{\text {sour }}=2.76 \times 10^{-5}, \mathrm{H} / \mathrm{k}=3.289 \times 10^{3}{ }^{\circ} \mathrm{K}
$$


Table 5.1. Material properties for gold single crystals

\begin{tabular}{lclc}
\hline Young's modulus & $78 \mathrm{GPa}$ & Yield stress & $78 \mathrm{MPa}$ \\
Poisson's ratio & 0.35 & Rate sensitivity factor & 0.01 \\
Reference strain rate & $0.001 / \mathrm{s}$ & Critical strain rate & $10^{4} / \mathrm{s}$ \\
Burgers vector & $3.0 \times 10^{10} \mathrm{~m}$ & $\begin{array}{l}\text { Reference stress interaction } \\
\text { coefficients } \mathrm{a}_{\mathrm{i}}(\mathrm{i}=1,12)\end{array}$ & 0.50 \\
\hline
\end{tabular}

From the MD nanoindentation simulations, (see for example, Schall and Brenner, 2004 and Hasnaoui, Derlet et al., 2004), which are based on a load-displacement input, we obtained different displacement profiles related to contact depths and indentation surface profiles. These MD displacement profiles were obtained from the application of contact forces as a function of displacement. We scaled these displacements as a function of the maximum normalized indentation depth. We normalized the predicted MD indentation depths (or applied displacements) by the specimen height, which would correspond to a normal strain. We then used this normal strain, which is scale invariant, to obtain displacement histories or indentation depths, as a function of the contact radius. Using this scaling scheme, we can then determine the maximum depth as a function of this applied strain, for the FEM microstructural model. It should also be noted that in this scaling relation we are essentially coarsening the grain-sizes. Specifically, if we assume, for the FEM models, the same aggregate size for the MD simulations as we do for the FEM models, we are applying the same strains in the FEM model as the MD model, but for larger sized grains. Once the desired depth is attained, the indentation, through this virtual indenter, can be completely unloaded.

As an example of our scaling technique, we use the MD simulations of Hasnaoui et al. (2004). For this MD simulation of nanoindentation, the average grain-size is approximately $12 \mathrm{~nm}$, the indenter diameter is $8 \mathrm{~nm}$, and the maximum applied indented 
depth is $2 \mathrm{~nm}$. Using this information for our FEM models, we arranged the 15 grains by having five horizontal and three vertical grains. We then calculated the ratio between the indenter size, the grain diameter, and the indentation depth to determine the applied strains as a function of the displacement or indentation depth. The summarized scaling information is given in Table 5.2, and it is shown schematically in Figure 5.1.

Table 5.2. Scaling of MD simulations to microstructural FEM models

\begin{tabular}{lcc}
\hline & MD & FEM \\
\hline $\begin{array}{l}\text { Number of grains } \\
\text { Grain-size }\end{array}$ & 15 & 15 \\
$\begin{array}{l}\text { Normalized length } \\
\text { (width/height) }\end{array}$ & $12 \mathrm{~nm}$ & $0.003 \mathrm{~mm}$ \\
$\begin{array}{l}\text { Indenter diameter } \\
\text { Maximum depth }\end{array}$ & 1.667 & 1.667 \\
$\begin{array}{l}\text { Normalized indenter size } \\
\text { (indenter diameter/grain-size) }\end{array}$ & $8 \mathrm{~nm}$ & $0.002 \mathrm{~mm}$ \\
$\begin{array}{l}\text { Normalized depth (maximum } \\
\text { depth/indenter diameter) }\end{array}$ & 0.667 & $0.0005 \mathrm{~mm}$ \\
$\begin{array}{l}\text { Strain } \\
\text { (maximum depth/height) }\end{array}$ & 0.25 & 0.667 \\
\hline
\end{tabular}

As shown in Table 5.2, we use the normalized indenter size and the normalized depth, which is the normal strain, as the desired indented depth for the FEM microstructural models. For different indentation depths, the ratio between indentation depth and specimen vertical length will obviously vary. In the FEM models, we used periodic boundary conditions with plane strain loading conditions and four-noded quadrilateral elements.

The scaling scheme, which is used to link the MD simulations with the FEM microstructural models, can be summarized as follows:

1. We obtain scaled displacements from the MD simulations as a function of the 
maximum normalized indentation depth;

2. The predicted MD displacements are normalized by the specimen height, which correspond to the normal strain or the normalized indented depth;

3. We can then determine the maximum depth as a function of this applied strain;

4. We then use this strain to obtain displacement histories, as a function of the contact radius to obtain the indented depth for the FEM models.

We can also use these scaling relations to investigate grain-size effects. In the first model we had an average grain-size of $0.003 \mathrm{~mm}$ for essentially square-shaped grains. If we change the grain diameter to $0.0022 \mathrm{~mm}$, we would have more grains for the same specimen dimensions. It should also be noted that with a cube orientation, a polycrystalline aggregate approaches the limiting case of a single crystal, hence for these orientations, the GB region should not be distinct from the grain interior. Strains, or normalized indented depths, for different grain-sizes and aggregates are given in Table 5.3.

Table 5.3. Grain-sizes and aggregates

\begin{tabular}{lcc}
\hline & Model 1 (15 grains) & Model 2 (28 grains) \\
\hline Grain-size & $0.003 \mathrm{~mm}$ & $0.0022 \mathrm{~mm}$ \\
$\begin{array}{l}\text { Normalized length } \\
\text { (width/height) }\end{array}$ & 1.677 & 1.759 \\
$\begin{array}{l}\text { Number of grains } \\
\text { Strain }\end{array}$ & 15 & 28 \\
(maximum depth/height) & $0.09 / 0.128 / 0.231$ & $0.09 / 0.128 / 0.231$ \\
\hline
\end{tabular}

\subsubsection{Incorporation of Scaling Effects in Microstructural FEM Models}

We used the scaling relations for the two different sized aggregates to investigate how inelastic deformation evolves at the microstructural scale for the cube oriented 
aggregates. The applied displacement history is shown in Figure 5.2. As seen in this figure, nodal displacements are applied the indented surface. The slope for loading and unloading paths are determined from Schall and Brenner (2004). The unloading slope is measured at the beginning of each step, which is consistent with experimental approaches. The slope in the unloaded region is approximately $20 \%$ higher than the slope in the loaded region. Hence, the slope in the unloaded region is stiffer than the slope in the loaded region (Figure 5.2). After a convergence analysis, 1455 quadrilateral elements were used for Model 1 and 2146 elements were used for Model 2.

As a virtual indenter for our FEM model, displacement profiles for each node, in the contact region, for different indentation depths, were applied. Each node was displaced along the loading and unloading step according to the strain that corresponds to the indentation depths. At the beginning of the indentation, only one node is displaced and adjacent nodes are also displaced as the time step increases (Table 5.4). Calculated axial distances between the center of the virtual indenter and the coordinate of each node are used for the displacement curve at each node. With this scheme the number of loaded nodes increases as the indentation depth increases (Figure 5.3).

Table 5.4. Number of nodes applied normalized displacement input for different indentation depth

\begin{tabular}{l|cc|cc|cc}
\hline Depth in MD (nm) & \multicolumn{2}{|c|}{3.5} & \multicolumn{2}{c|}{5} & \multicolumn{2}{c}{9} \\
\hline Model type & 1 & 2 & 1 & 2 & 1 & 2 \\
No. of loaded nodes & 15 & 13 & 17 & 21 & 27 & 39 \\
Max. depth (mm) & 0.000875 & 0.000859 & 0.00125 & 0.00123 & 0.00225 & 0.00221 \\
Normalized input & \multicolumn{2}{|c|}{0.09} & \multicolumn{2}{c|}{0.128} & \multicolumn{2}{c}{0.231} \\
\hline
\end{tabular}




\subsection{Validation of Hierarchical Modeling Scheme by Comparison of Hardness of Gold Single Crystals with Experimentally Obtained Values}

For the two different models, nanoindentation was simulated for three different indentation depths. Using the contact area as defined in Figure 5.3 (variable 2a), the hardness value was calculated for the gold single crystals. For the hardness calculation contact area, $2 a$, is defined according to each indentation depth, i.e. indenter size, by measuring the distance from the projected diameter of the virtual indenter at the maximum indentation depth. Figure 5.4 shows the hardness values for different indentation depths. Of course, the hardness values vary along the indentation depth. However, for both models, the hardness is approximately $2.0 \mathrm{GPa}$ when the indentation depth corresponds to a normal strain of $23.1 \%$. This value closely coincides with the experimental values obtained by McCann (2004). They used a diamond Berkovich indenter on a gold (111) surface and the average measured hardness was 2.05 GPa with the standard deviation of 0.62 GPa. Li, Nardi et al. (2005) also used the indentation-projected area to calibrate the hardness. The measured hardness of the gold thin film that was used in their Berkovich shaped nanoindentern is $2.1 \pm 0.3 \mathrm{GPa}$. Gerberich, Tymiak et al. (2002) also measured the hardness of $<100>$ gold single crystals by using a three-sided Berkovich indenter. The results from their experiment show that hardness of gold single crystal is approximately $2.0 \mathrm{GPa}$ with $\pm 0.60 \mathrm{GPa}$. Corcoran, Colton et al. (1997) concluded that the hardness of gold single crystals, as measured by different nanoindenters, varied from $650 \mathrm{MPa}$ to $2 \mathrm{GPa}$. Smith and Zheng (2000) also used nanondenation to obtain hardness values of $0.85 \pm 0.01 \mathrm{GPa}$ for gold single crystals at room temperature. Since our predicted hardness values clearly coincide with these experimentally measured values for pure gold, this further confirmed that our scaling scheme for our hierarchical model was physically consistent and accurate with experimentally obtained 
measurements and observations related to nanoindentation and hardness for crystalline f.c.c. materials.

\subsection{Summary and Conclusions}

Our hierarchical scheme, which is based on scaling the specimen and indenter size, and indentation depth, can be used to link MD simulations with the FEM microstructural models to obtain new and significantly improved predictions of microstructural behavior at physical scales that are commensurate with inelastic behavior. We validated this hierarchical scheme, by calculating the hardness values of crystalline gold, and our predictions accurately matched a number of experimentally obtained measurements.

The hierarchical model is based on scaling the grain and indenter size and indentation depth, such that normalized indentation depths are obtained and are based on the displacement MD profiles. This approach provides a methodology to link molecular level simulations, with a FEM and constitutive formulation that can then be used to ascertain the effects of dislocation-density evolution, grain-size and GB effects, and crystalline structure on indentation effects on scale effects that can track indentation effects at long-range temporal and spatial scales. Hence, fundamental crystalline behavior can then be predicted as a function of hydrostatic pressures, inelastic strains, slip-system activity and orientation, and GB effects.

The present study provides a validated scheme for linking MD models to continuumbased microstructural finite-element formulations, such that critical material aggregate behavior can be tracked from a nucleation event to a desired microstructural evolution mode. Different aggregate and GB arrangements will be investigated in the incoming chapters to further ascertain how different material variability can be handled by the present scheme. 


\section{References}

Corcoran, S. G., R. J. Colton, et al. (1997). "Anomalous plastic deformation at surfaces: Nanoindentation of gold single crystals." Physical Review B 55(24): 16057-16060.

Gerberich, W. W., N. I. Tymiak, et al. (2002). "Interpretations of indentation size effects." Journal of Applied Mechanics-Transactions of the Asme 69(4): 433-442.

Hasnaoui, A., P. M. Derlet, et al. (2004). "Interaction between dislocations and grain boundaries under an indenter - a molecular dynamics simulation." Acta Materialia 52(8): 2251-2258.

Li, X. D., P. Nardi, et al. (2005). "Direct nanomechanical machining of gold nanowires using a nanoindenter and an atomic force microscope." Journal of Micromechanics and Microengineering 15(3): 551-556.

McCann, M. M. (2004). Nanoindentation of Gold Single Crystal. Material Science and Engineering. Blacksburg, Virginia Polytechnic Institute and State University.

McCann, M. M., and Corcoran, S.G. (2004). Nanoindentation behavior of gold single crystals. Material Research Society Symposium 2003 Fall meeting, Boston, MA U.S.A., Material Research Society.

Schall, J. D. and D. W. Brenner (2004). "Atomistic simulation of the influence of pre-existing stress on the interpretation of nanoindentation data." Journal of Materials Research 19(11): 3172-3180.

Smith, J. F. and S. Zheng (2000). "High temperature nanoscale mechanical property measurements." Surface Engineering 16(2): 143-146.

Pamula, V. K., A. Jog, et al. (2001). "Mechanical property measurement of thin-film Gold using thermally actuated bimetallic cantilever beams. " Modeling and Simulation of Microsystems: 410-413 


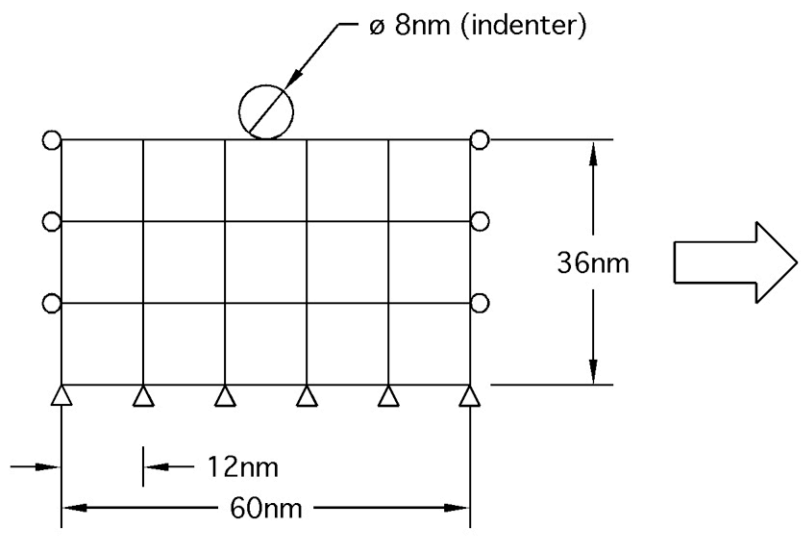

(a)

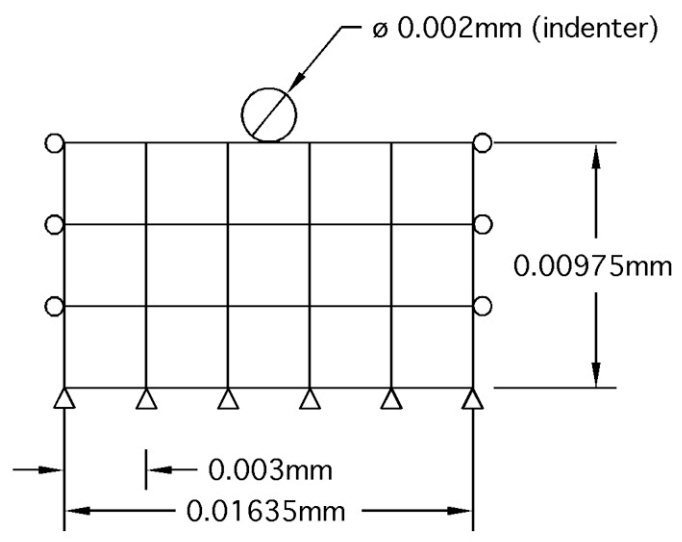

(b)

Figure 5.1. Schematic model for finite-element analysis (scaling MD to FEA)

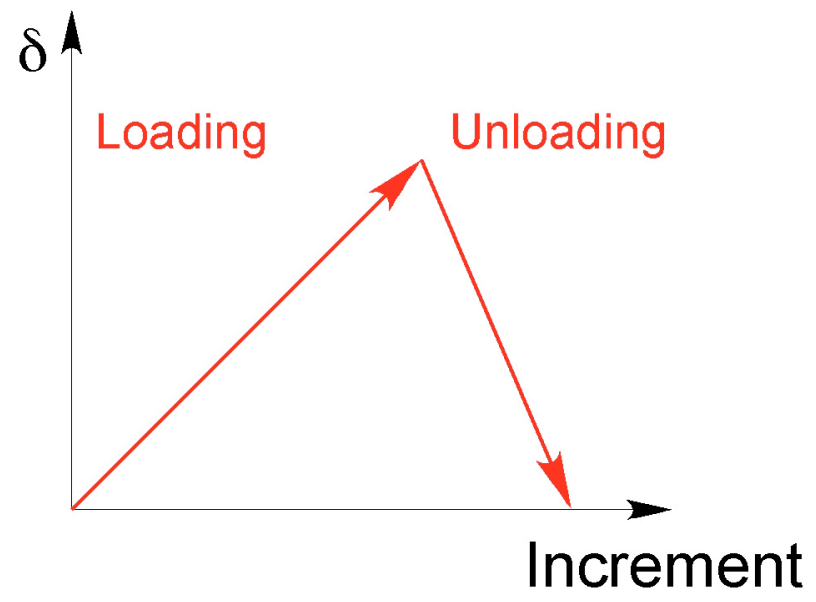

Figure 5.2. Simplified loading-unloading curve for FEA 


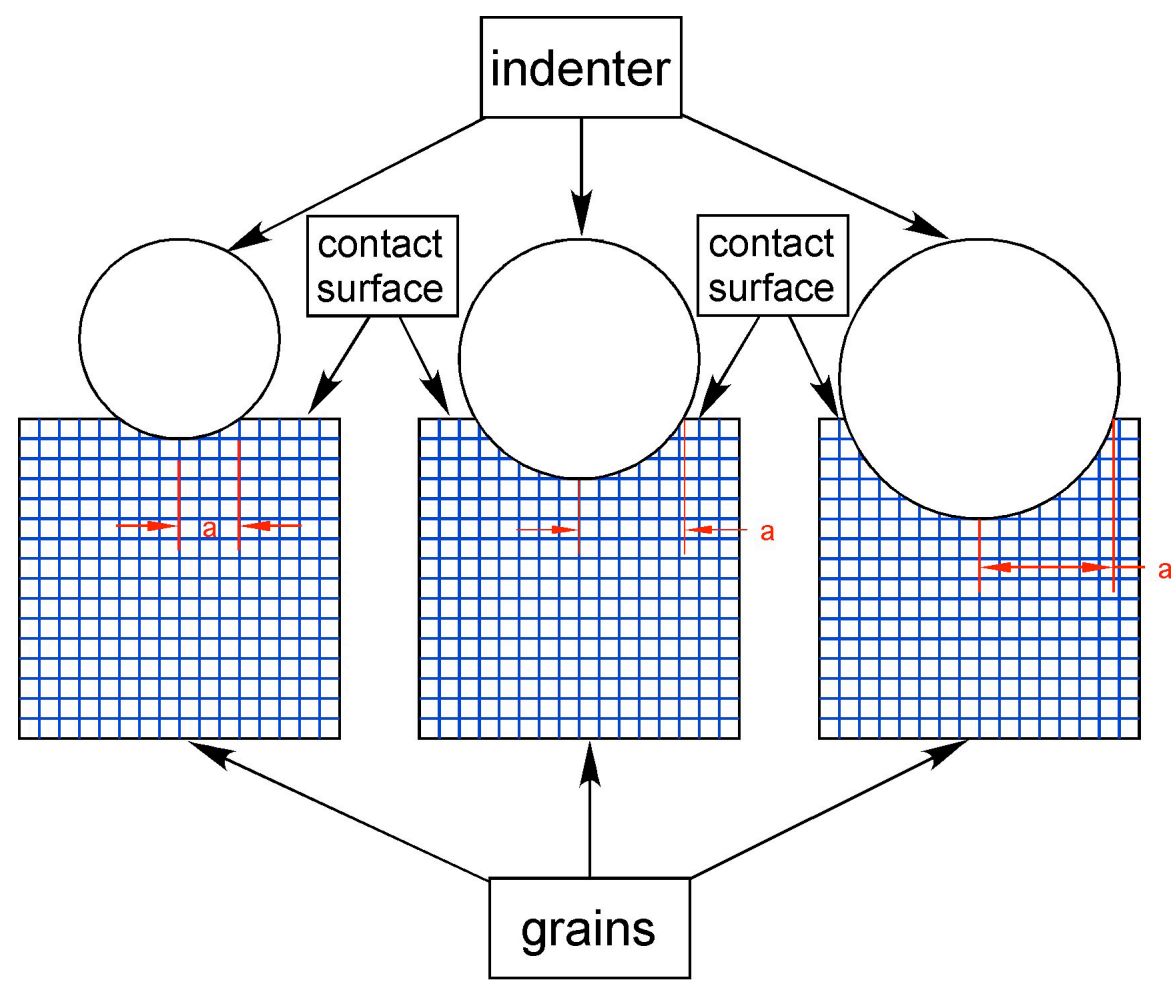

Figure 5.3. Schematic of indentation according to indenter size and indentation depth

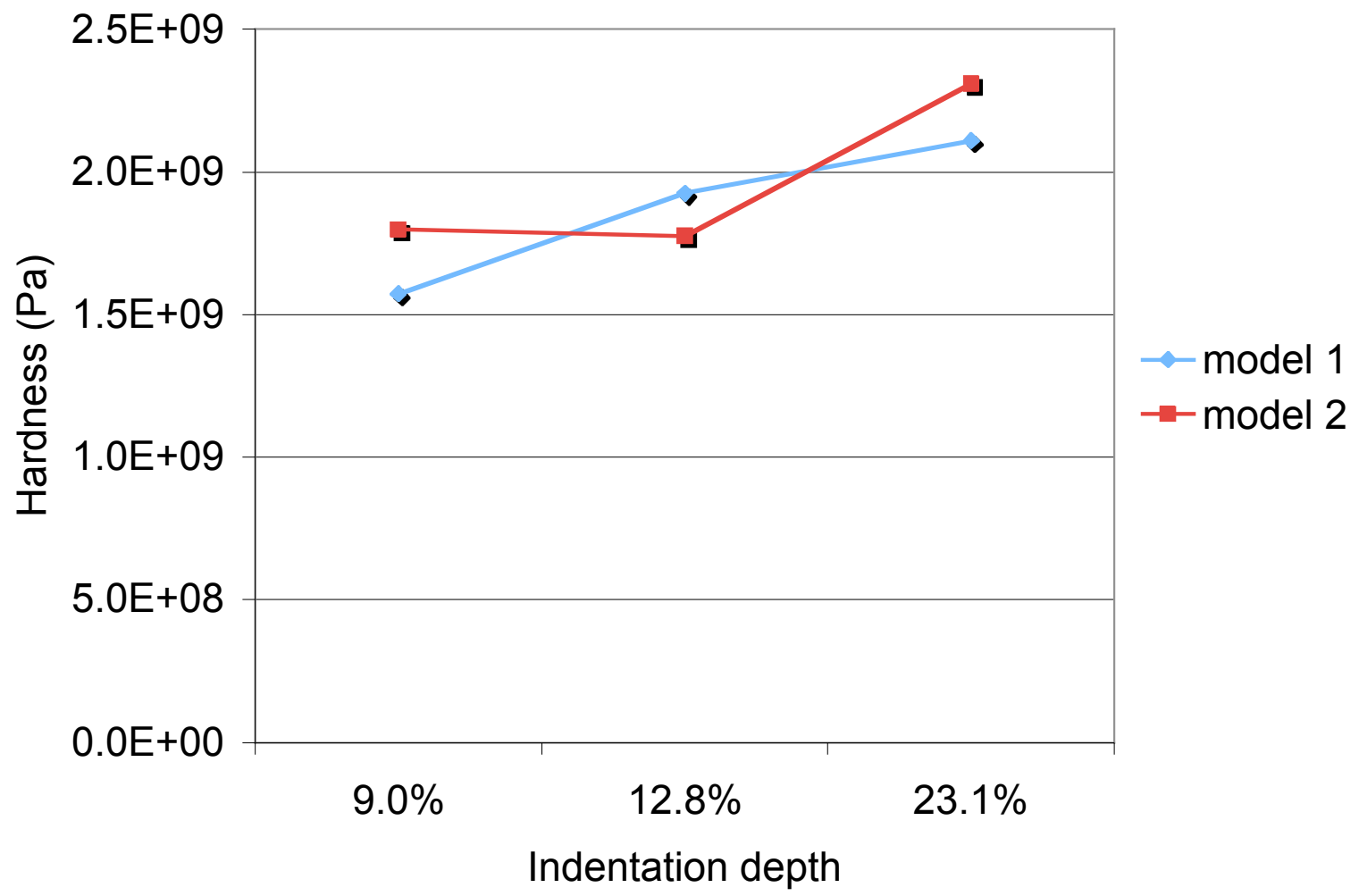

Figure 5.4. Hardness of gold single crystals by FEA 


\section{CHAPTER 6}

\section{NANOINDENTATION AND MICROSTRUCTURAL EVOLUTION OF CUBE ORIENTED CRYTSALLINE AGGREGATES}

The multiple-slip crystal plasticity dislocation-density based constitutive formulation, the finite-element computational scheme, and the GB interfacial interaction model formulations were applied to investigate the nanoindentation of f.c.c. gold aggregates with a cube orientation of Euler angles of $(0,0,0)$ for all the grains. As discussed earlier, two different sized crystalline aggregates with fifteen and twenty-eight grains were employed for these investigations. The load-displacement profile for the virtual indenter, which was discussed in Chapter 5, was used (Figure 5.2). The material properties that are used here are representative of polycrystalline gold (McCann, 2004 and Pamula et al., 2001). The grain bulk properties are given in Table 5.1.

\subsection{Overall Aggregate Response and Slip-system Activity during Global Loading}

The global stress-strain response for both aggregates is shown in Figure 6.1. As seen from this figure, as the compressive nominal strain increases, the stress increases for both crystalline aggregates. The compressive nominal strains here correspond to the summation of the normalized displacements applied at the nodes at the top surface, and the global stresses are the summation of the nodal forces corresponding to the undeformed area. Since increases in the nominal strain also correspond to increases in the indentation depth, this behavior is consistent with experimental observations (see, for example, Gerberich, Tymiak et al. 2002), which indicates that the resistance to indentation increases as the indentation depth increases. 
As also seen from the overall stress-strain response, both aggregates also have approximately the same overall average stress response during the loading portion corresponding to indentation. The response, for each aggregate, differs during unloading due to the residual strains, which is related to the number of indented grains. Since the grain-sizes and the aggregate size are known, we calculated that at a nominal strain of $9 \%$, only one grain was indented for the smaller-sized aggregate. As the indentation depth increases, the number of indented grains also increased. Hence, at a nominal strain of $23.1 \%$, the number of indented grains increased to three for Model 1 (the smaller-sized aggregate).

As the overall stress-strain response also indicates, during indentation, there are small local unloading stages, as indicated in Figure 6.1. As experimental studies have indicated (Suresh, Nieh et al., 1999 and Knapp, 2003), these unloading stages, which occur during indentation, can occur due to the initiation of slip and dislocation-densities on different slip-system. We investigated to see if this was the case for these simulations. For Model 1 (the smaller-sized aggregate), at a nominal strain of 9\%, there were four active slipsystems that were most active before this local unloading during indentation. (see Table 4.1 for slip-system identification). Slip-systems 3, 4, 9, and 10 were most active before unloading but after unloading, slip-systems 1, 6, 7, and 12 were most active. The slip-system activities for both models are summarized in Table 6.1. This same type of slip-system activity occurred for the Model 2, but at different nominal strains. This could be due to that more grains are indented for the larger-sized aggregate, and that consequently more slip-systems are operative within more grains. These results indicate that slip-system and immobile dislocation-density activities, at specific nominal strains, are initiated during indentation, but 
that different slip-systems and immobile dislocation-densities are activated due to lattice rotations and accumulations of plastic strains and pressures in indented regions and grains.

Table 6.1. Slip-system activity variation before and after local unloading

\begin{tabular}{ccc}
\hline Normalized Indented Depth & $\begin{array}{c}\text { Most active slip-systems } \\
\text { before load-drop }\end{array}$ & $\begin{array}{c}\text { Most active slip-systems } \\
\text { after load-drop }\end{array}$ \\
\hline Nominal Strain 9\%, Model 1 & $3,4,9,10$ & $1,6,7,12$ \\
Nominal Strain 12.8\%, Model 1 & $1,6,7,12$ & $1,6,7,12$ \\
Nominal Strain 23.1\%, Model 1 & $3,4,9,10$ & $3,4,9,10$ \\
Nominal Strain 9\%, Model 2 & $3,4,9,10$ & $1,6,7,12$ \\
Nominal Strain 12.8\%, Model 2 & $3,4,9,10$ & 1,7 \\
Nominal Strain 23.1\%, Model 2 & 2,8 & 6,12 \\
\hline
\end{tabular}

\subsection{Immobile Dislocation-density Activities, Inelastic Behavior, and Local Stress and Pressure Response}

To further understand how activities on different slip-system affect microstructural behavior during indentation and unloading, we investigated immobile dislocation-density activities on the most active slip-system at different normalized indented depths for both aggregates (a summary is given in Table 6.2).

Table 6.2. List of most active slip-systems at maximum normalized depths

\begin{tabular}{cccc}
\hline $\begin{array}{c}\text { Normalized } \\
\text { Indented Depth }\end{array}$ & Most active slip-systems & $\begin{array}{c}\text { Normalized } \\
\text { Indented Depth }\end{array}$ & Most active slip-systems \\
\hline $9 \%$, Model 1 & 6,12 & $9 \%$, Model 2 & 2,8 \\
$12.8 \%$, Model 1 & 2,8 & $12.8 \%$, Model 2 & 1,7 \\
$23.1 \%$, Model 1 & $3,4,9,10$ & $23.1 \%$, Model 2 & 6,12 \\
\hline
\end{tabular}

At a normalized indentation depth of 9\%, for Model 1, slip-systems 6 and 12 have the same immobile dislocation-density values, and both immobile dislocation-densities evolve to $27.1 \%$ of the saturated value for both slip-systems, and hence these two slip- 
systems have a normalized value of approximately $54 \%$ of saturation value (Figure 6.2a). The total immobile dislocation-density, which is the summation of all immobile dislocationdensities on all slip-systems, was also calculated. From this value, we knew that the total immobile dislocation-density for all the slip-system is $1.29 \times 10^{16} \mathrm{~m}^{-2}$ (Figure 6.2b). This indicates that there is significant immobile dislocation-density activity, since this value is $1.29 \times 10^{6}$ times greater than the initial immobile dislocation-density. As also seen from the contours, this activity occurs throughout the aggregate, and is not localized near the indented region, which immobile dislocation-density activity is not localized around the indented surface. This is significant, since this further underscores the importance of modeling on scales beyond those associated with MD simulations.

We also investigated the immobile dislocation-density activity when the virtual indenter is unloaded. The immobile dislocation contour densities, before the final unloading step, for slip-systems 6 and 12, and total immobile dislocation-density are shown in Figures 6.2c-d. As seen from these figures, it is approximately $1.87 \times 10^{6}$ times greater than the initial immobile dislocation-density $\left(\rho_{i m}=1.87 \times 10^{16} \mathrm{~m}^{-2}\right)$, for most active slip-systems, and $1.87 \times 10^{7}\left(\rho_{i m}=1.87 \times 10^{17} \mathrm{~m}^{-2}\right)$ times greater than the initial immobile dislocation-density , for total slip-systems, before the final unloading step. From these results, the immobile dislocation-density of the most active slip-systems accounts for $42 \%$ of all the immobile dislocation-density activities, while at the final stage of indenter unloading; the immobile dislocation-density of the most active slip-systems accounts for $20 \%$ of the immobile dislocation-density activities. This indicates that as unloading occurs, more slip-systems are activated, and the deformation is no longer dominated by two slip-systems. As these results also indicate, the spatial distribution of the immobile dislocation-densities, corresponding to 
the most active slip-system, is highly localized at the final loading and unloading stages.

The plastic strain and pressure distributions at the maximum loading and unloading points are shown in Figure 6.3. As evident from these contours, the maximum immobile dislocation-densities are accumulated at the location that the pressure has the highest normalized (all pressures are normalized by the yield stress) value of -24 at the final loading stage and 40.3 at the final unloading stage. The maximum pressures and immobile dislocation-densities also occur within the accumulated plastic zone. The maximum accumulated plastic strain is 1.81 at the final loading stage, and it is 2.79 at the final unloading stage. As seen from the plastic zone accumulation, it is a continuous region corresponding to all the different plastic activities, as opposed to the discrete and discontinuous regions corresponding to the immobile dislocation-density activities. As also seen from these figures, the highest pressures are on either side of the virtual indenter. These high pressures may also be a contributing factor to the accumulated immobile dislocationdensities and plastic strains. The pressures and the plastic strains are symmetric on either side of the indenter, and this may be due to the cube orientation used for the aggregates. Furthermore, these high pressures can result in the activation of different slip-system, in different grains (see, for example, Hasnaoui, Derlet et al., 2004), since these very high pressures exceed the reference stresses on the different slip-systems.

\subsection{Effects of Increased Indentation Depths}

To understand how increased indentation depths affects microstructural behavior, we increased the normalized indentation depths to $12.8 \%$ and $23.1 \%$ for the smaller-sized 
aggregate, Model 1 . The contours of immobile dislocation-density (for the most active slipsystem), plastic strain, and pressures, are shown in Figures 6.4-6.7. At an indentation depth of $12.8 \%$, the most active slip-systems of 2 and 8 have an accumulated immobile dislocationdensity of $51 \%$ of the saturated value at each system. The total immobile dislocation-density of all slip-system at $12.8 \%$ indentation was $2.4 \times 10^{16} \mathrm{~m}^{-2}$ (Figures 6.4a-b), and this is approximately twice as high as the immobile dislocation-densities for the 9\% normalized depth. As noted earlier, this is consistent with experimental observations and measurements, since more grains are indented as the indented depth increases.

At the final stage of unloading, the immobile dislocation-densities of the two most active slip-systems (system 2 and 8 ) are $3.1 \times 10^{16} \mathrm{~m}^{-2}$ (Figure $6.4 \mathrm{c}$ ), and it is $2.3 \times 10^{17} \mathrm{~m}^{-2}$ for total immobile dislocation-densities of all slip-systems (Figure 6.4d). Hence, the two most active slip-systems account for $42.4 \%$ of the total immobile dislocation-density activity, at the maximum loading stage, which is comparable with the percentage of active slip-system for a normalized depth of $9 \%(42 \%)$. However, for the final unloading stage, the most active slip-system account for $26.2 \%$ of all the immobile dislocation-density activity, which is higher than the case for the normalized depth of $9 \%$ (20\%). This indicates that slip-systems are less activated for this normalized depth. This is also due to the higher residual strains, during unloading, that are associated with this deeper indented depth (cf. Figure 6.1). The accumulated plastic strains, at the final stage of loading, are 2.03, which is also higher than that of the $9 \%$ case (1.81) (Figure 6.5a). At the final stage of loading, there were compressive pressures throughout most of the specimen, with a maximum normalized value of -25 . For unloading, the pressure had a maximum normalized value of 46 . This pressure, which is 
significantly higher than that of the $9 \%$ case, is due to the larger number of indented grains and immobile dislocation-density activities (Figures 6.5c-d).

At a normalized indentation depth of $23.1 \%$ for Model 1, the immobile dislocationdensities were most active on systems 3,4,9, and 10 (Figure 6.6a). There was equal activity on all of these systems, and the sum of these four systems was $8.04 \times 10^{16} \mathrm{~m}^{-2}$, and accounted for $95 \%$ of the total immobile dislocation-density evolution. This activity pertaining to the most active slip-system was significantly higher than those pertaining to the smaller indented depths. The total immobile dislocation-density for all slip-system was $8.45 \times 10^{16} \mathrm{~m}^{-2}$ (Figure 6.6b). At the final point of unloading, the immobile dislocation-density was $4.55 \times 10^{16} \mathrm{~m}^{-2}$ for each of these active slip-systems, and it was $4.4 \times 10^{17} \mathrm{~m}^{-2}$ for the total immobile dislocationdensities corresponding to all slip-system (Figures 6.6c-d). These active slip-systems accounted for $41 \%$ of the total immobile dislocation-density activity at the final stage of unloading, which is a much higher percentage than that associated with the smaller indented depths (9\% depth: $20 \%, 12.8 \%$ depth: $26.2 \%$ ). The accumulated plastic strains were also significantly higher than those associated with the smaller indented depths, and the maximum value was approximately 2.60 at the maximum loading stage (Figure 6.7a). The pressures were also higher at this indented depth, as compared with the smaller indented depths, with a maximum value of 53.5 at the final stage of unloading. But at this deeper indented depth, the pressures were more diffuse as compared with the localized distribution that was seen at the smaller indented depths (Figure 6.7c). 


\subsection{Effects of Larger-sized Aggregate}

We investigated the effects of increasing the aggregate size by increasing the aggregate size to 28 grains (Model 2). At a normalized indentation depth of $9 \%$, slip-systems 2 and 8 both accounted for $28 \%$ of immobile dislocation-densities (Figure 6.8a). This is significantly lower than the smaller-sized aggregate, where the active slip-system accounted for approximately $40 \%$ of the immobile dislocation-density activity. Hence, for a larger-sized aggregate, more slip-systems are active, and the most active slip-system are not as dominant as they were for the smaller-sized aggregate. The total immobile dislocation-density for all active slip-system is $1.03 \times 10^{16} \mathrm{~m}^{-2}$ (Figure 6.8b). As noted earlier , as the aggregate size is increased, more grains are indented.

The immobile dislocation-densities, for the final unloading stage, for the most active slip-system, slip-systems 2 and 8 are shown in Figure $6.8 \mathrm{c}$ and the total immobile dislocation-density for all slip-systems is given in Figure $6.8 \mathrm{~d}$. The two most active slipsystems are $1.47 \times 10^{16} \mathrm{~m}^{-2}$ at the final unloading stage, and it is $8.56 \times 10^{16} \mathrm{~m}^{-2}$ at unloading for total immobile dislocation-densities. At the final stage of unloading, the most active slipsystem accounted for $34 \%$ of all the immobile dislocation-density activities, which is significantly higher than the smaller-sized aggregate, where the active slip-system accounted for $20 \%$ of the immobile dislocation-density activity. This indicates that as the aggregate is unloaded, and as larger residual strains accumulate for the larger-sized aggregate (Figure 6.1), these higher residual strains result in more active slip-system in comparison with the smaller-sized aggregates. 
To further understand what the effects of increasing the aggregate size is, we investigated the distributions of the pressure and accumulated plastic strains at the maximum loading and unloading points (Figure 6.9). The maximum immobile dislocation-densities are accumulated at the location of the maximum pressures, which have normalized values of -26.08 at the final loading point and 35.2 at the final unloading point. The highest pressures are on either side of the virtual indenter. These high pressures are a contributing factor to the accumulated immobile dislocation-densities and plastic strains, since these high pressures are much greater than the yield stresses, and this would contribute to inelastic activities in these regions (see, for example, Lilleodden, Zimmerman et al. 2003). The pressures and the immobile dislocation-densities are also symmetric, and this are due to the cube orientation used for the aggregates. The maximum plastic strains are also accumulated at these points, and they are 1.72 at the final loading point and 2.51 at the final unloading point. These are smaller than the larger grain-size, Model 1 at the same normalized indentation depth, and they also correspond with the small immobile dislocation-densities of the larger-sized aggregate (smaller grain-size).

Indentation depths were also increased to $12.8 \%$ and $23.1 \%$ to compare indentation depth effects for different aggregate sizes. The contours of immobile dislocation-density (for the most active slip-system), plastic strain, and pressures are given in Figures 6.10-6.13. Slipsystems 1 and 7 have the same highest normalized immobile dislocation-density value of $62 \%$ of the saturated value (Figure 6.10a) and the total immobile dislocation-density of all slip-systems is $3.1 \times 10^{16} \mathrm{~m}^{-2}$ at normalized indentation depth of $12.8 \%$ (Figure $6.10 \mathrm{~b}$ ). This total immobile dislocation-density is approximately more than three times as high as the immobile dislocation-densities for the $9 \%$ normalized depth. 
At the final stage of unloading, the immobile dislocation-density of most active slipsystem 1 and 7 is $2.7 \times 10^{16} \mathrm{~m}^{-2}$ (Figure $6.10 \mathrm{c}$ ) and $3.3 \times 10^{17} \mathrm{~m}^{-2}$ for the total immobile dislocation-densities (Figure 6.10d). Hence, the active slip-system account for approximately $41 \%$ of the total activities, as compared with the $28 \%$ of immobile dislocation-density activities associated with $9 \%$ case. However, this activity decreases to $16.7 \%$ for the final unloading step. This indicates that more slip-systems are activated during unloading as the indentation depth increases. As the indentation depth increases, the plastic strain increases, and it attains a maximum of 2.07 (Figure 6.11a) and the pressure attain a maximum value of -29.48 at final loading step (Figure 6.11c).

At an indented normalized depth of $23.1 \%$, slip-system 6 and 12 are the most active at the final loading point with each having an immobile dislocation-density value of value of $2.27 \times 10^{16} \mathrm{~m}^{-2}$ (Figure $6.12 \mathrm{a}$ ). The sum of these two systems accounts for $42.8 \%$ of the total immobile dislocation-density. The total immobile dislocation-density for all slip-systems was $1.06 \times 10^{17} \mathrm{~m}^{-2}$ (Figure 6.12b). At the final unloading, the immobile dislocation-density is $1.77 \times 10^{17} \mathrm{~m}^{-2}$ for each of these slip-systems (Figure $6.12 \mathrm{c}$ ), and the total immobile dislocation-density is $1.62 \times 10^{18} \mathrm{~m}^{-2}$ (Figure 6.12d). At the unloading point, the active slipsystem account for approximately $22 \%$ of the immobile dislocation-density activity. As noted earlier, the most active slip-system for the smaller aggregate (Model 1 with $23.1 \%$ depth) accounted for $41 \%$ of the immobile dislocation-density activities. The smaller activity is due to that in the larger aggregate, more slip-systems are activated as more grains are indented during the loading stage. These slip-systems remain active during unloading and hence slipsystem dominance may decrease, as residual strains also increase. The accumulated plastic strains and pressures also increased as the indentation depth increased. The maximum plastic 
strain is 2.54 at the maximum indentation point (Figure 6.13a) and the maximum pressure attains a value of -34.06 (Figure $6.13 \mathrm{c}$ ). The spatial distributions are similar with those corresponding to the smaller indented normalized depths.

\subsection{Summary and Conclusions}

This study provides a detailed understanding of the interrelated physical mechanisms and behavior that result in material behavior under nanoindentation in polycrystalline aggregates with a cube orientation. A multiple-slip rate-dependent crystalline constitutive formulation that is coupled to the evolution of mobile and immobile dislocation-densities and specialized computational schemes has been developed to investigate microstructuallyinduced plasticity due to nanoindentation of f.c.c. gold aggregates with a cube orientation. The combined effects of mobile and immobile dislocation-densities and accumulated plastic strain have been used to characterize material behavior in f.c.c. polycrystalline aggregates.

We first investigated the slip-system activity variation before and after the load-drop during the global loading steps. From our predictions, we have shown that slip-system and immobile dislocation-density activities, at specific normalized depths, are initiated during indentation, and that different slip-systems and immobile dislocation-densities are activated due to lattice rotations and accumulations of plastic strains and pressures in indented regions and grains. From our simulations, we were able to identify when and how immobile dislocation-densities and slip-systems are activated at the maximum loading points and at the unloading point. We related these activities to the accumulation of pressures and plastic strains at different aggregate locations. 
By increasing the indentation depth, we were also able to determine that both the immobile dislocation-densities and plastic strains increase with significant increases in local pressures. As the indentation depth was increased, the pressures increased, and there were some negative pressures near the top region of the aggregate, which would indicate local unloading and pressure relieve, since the pressures were attaining physically limiting values. Generally at higher pressures, higher immobile dislocation-densities were localized and also higher inelastic strains were accumulated throughout the aggregate. The smaller aggregate generally had less immobile dislocation-density activities in comparison with the larger-sized aggregate. This is a direct consequence that fewer grains are indented for a smaller-sized aggregate, and hence fewer slip-systems in fewer grains can be activated.

This study underscores the importance of using a dislocation-density based formulation and a hierarchical modeling scheme that can result in a comprehensive understanding and prediction of the effects of multiple-slip inelastic deformation and dislocation interactions and evolution of finite-strain deformation in crystalline materials on a physical scale that can relate critical behavior at the nano-scale to the microstructural scale. 


\section{References}

Gerberich, W. W., N. I. Tymiak, et al. (2002). "Interpretations of indentation size effects." Journal of Applied Mechanics-Transactions of the Asme 69(4): 433-442.

Hasnaoui, A., P. M. Derlet, et al. (2004). "Interaction between dislocations and grain boundaries under an indenter - a molecular dynamics simulation." Acta Materialia 52(8): 2251-2258.

Knapp, J. A., Follstaedt, D.M., and Myers, S.M. (2003). "Evaluating Micromechanical Properties at Surfaces using Nanoindentation with Finite-element Modeling." International Journal of Damage Mechanics 12(4): 377-387.

Lilleodden, E. T., J. A. Zimmerman, et al. (2003). "Atomistic simulations of elastic deformation and dislocation nucleation during nanoindentation." Journal of the Mechanics and Physics of Solids 51(5): 901-920.

McCann, M. M., and Corcoran, S.G. (2004). Nanoindentation behavior of gold single crystals. Material Research Society Symposium 2003 Fall meeting, Boston, MA U.S.A., Material Research Society.

Suresh, S., T. G. Nieh, et al. (1999). "Nano-indentation of copper thin films on silicon substrates." Scripta Materialia 41(9): 951-957.

Pamula, V. K., A. Jog, et al. (2001). "Mechanical property measurement of thin-film Gold using thermally actuated bimetallic cantilever beams. " Modeling and Simulation of Microsystems: 410-413 


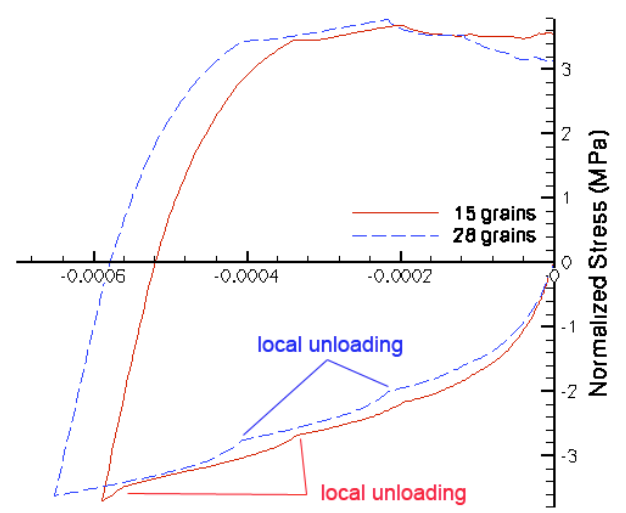

Nomınal Straın

(a)

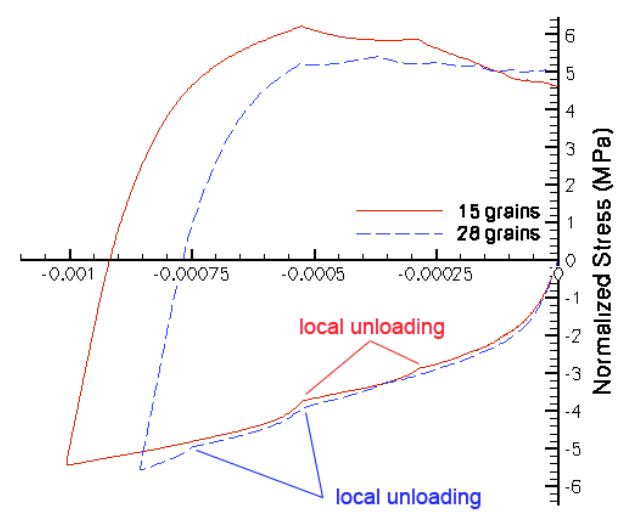

Nomınal Straın

(b)

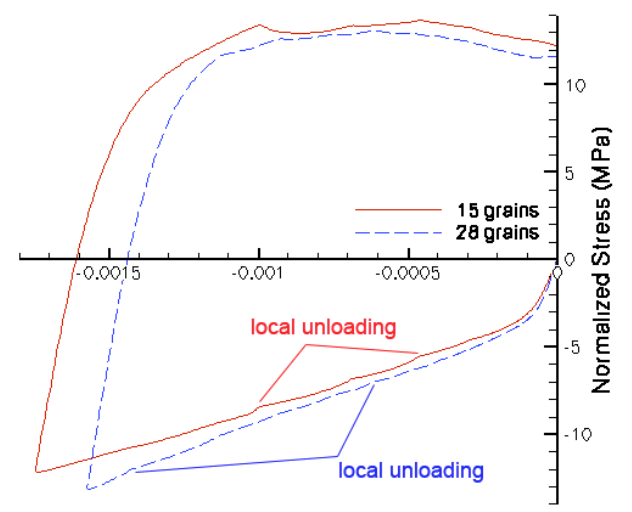

Nomınal Straın

(c)

Figure 6.1. Stress-strain curves for gold single crystals with different normalized indented depths and aggregate sizes (a) $9 \%$ (b) $12.8 \%$ (c) $23.1 \%$ 


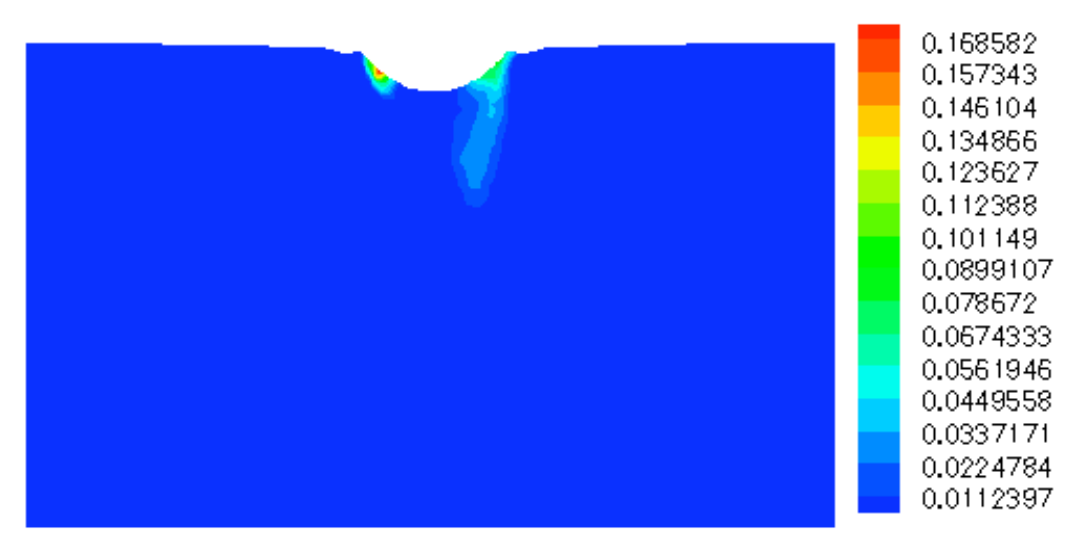

(a)

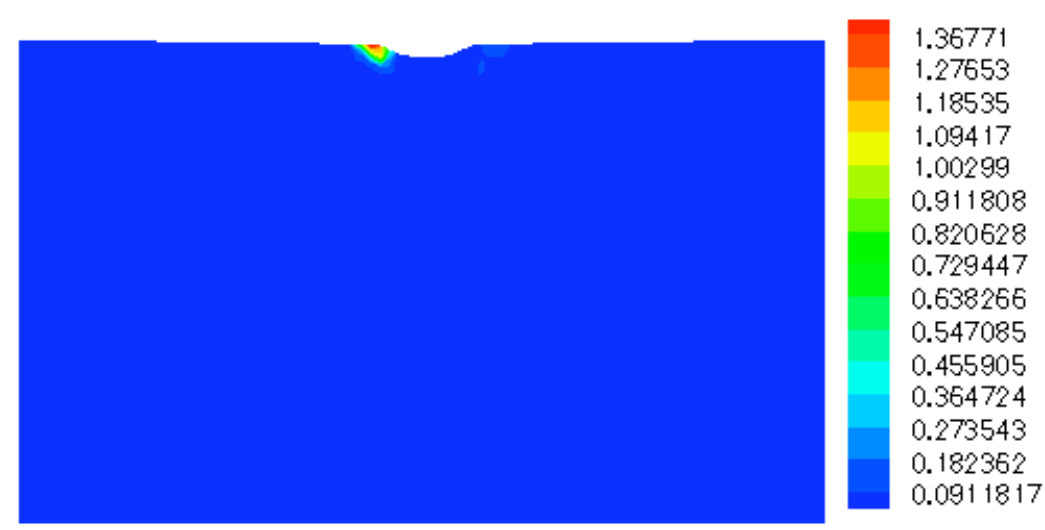

(c)

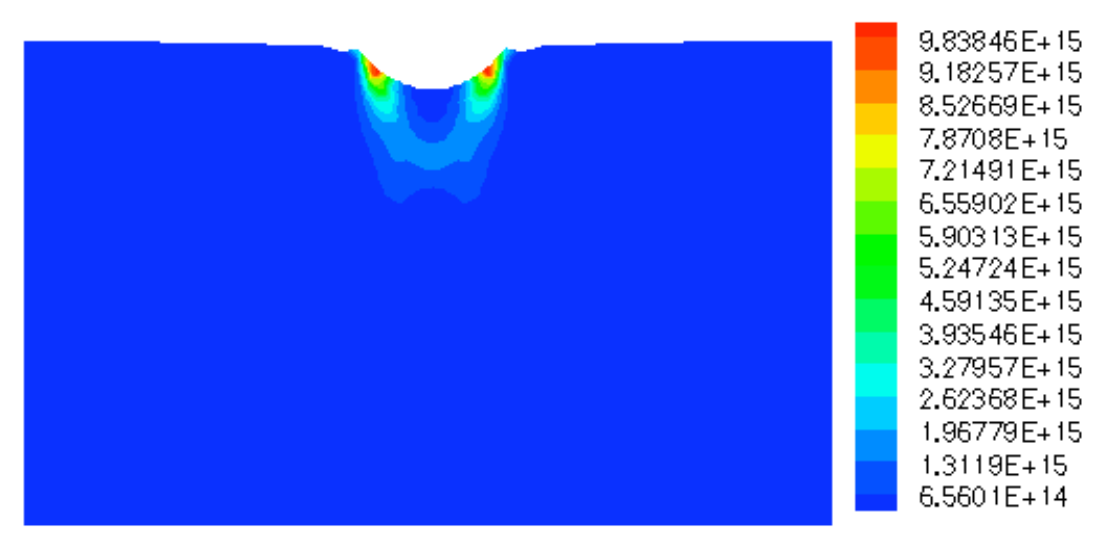

(b)

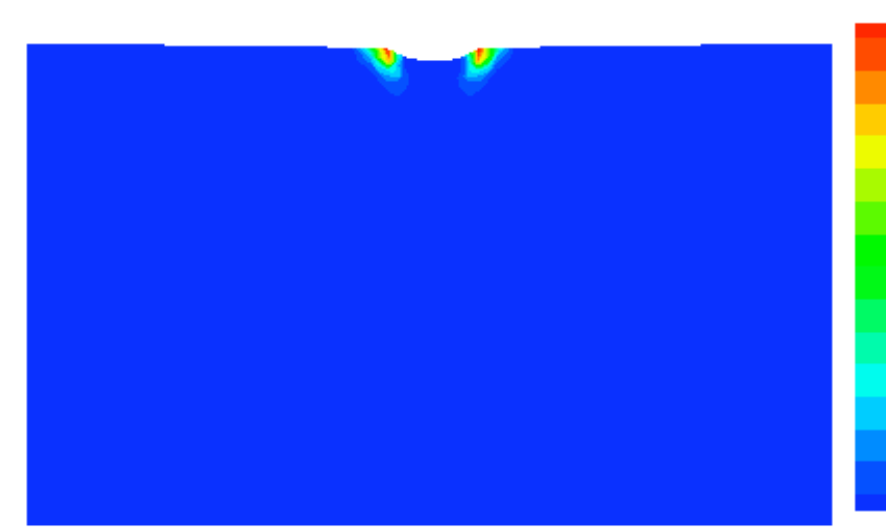

1. $19098 \mathrm{E}+17$ $1.11158 \mathrm{E}+17$ $1.03218 \mathrm{E}+17$ 9.52783E+16 $8.73385 E+16$ $7.93986 \mathrm{E}+16$ $7.14588 \mathrm{E}+16$ $7.14588 \mathrm{E}+16$ $5.55791 \mathrm{E}+16$ 4. $76392 \mathrm{E}+16$ $3.96994 \mathrm{E}+16$ $3.17595 \mathrm{E}+16$ $2.38197 \mathrm{E}+16$ $1.58798 \mathrm{E}+16$ (d)

Figure 6.2. Immobile dislocation-density at final loading step for system 6 and 12 normalized by saturation (a), total immobile dislocation-density at final loading step non-normalized (b), just before unloading step for system 6 and 12 normalized by saturation (c), and total immobile dislocation-density just before unloading step non-normalized (d). (Model 1 with 9\% indentation) 


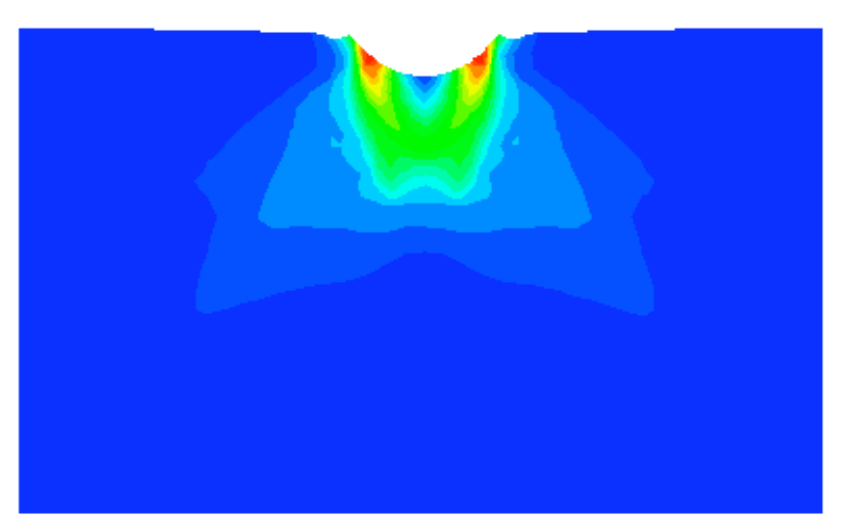

(a)

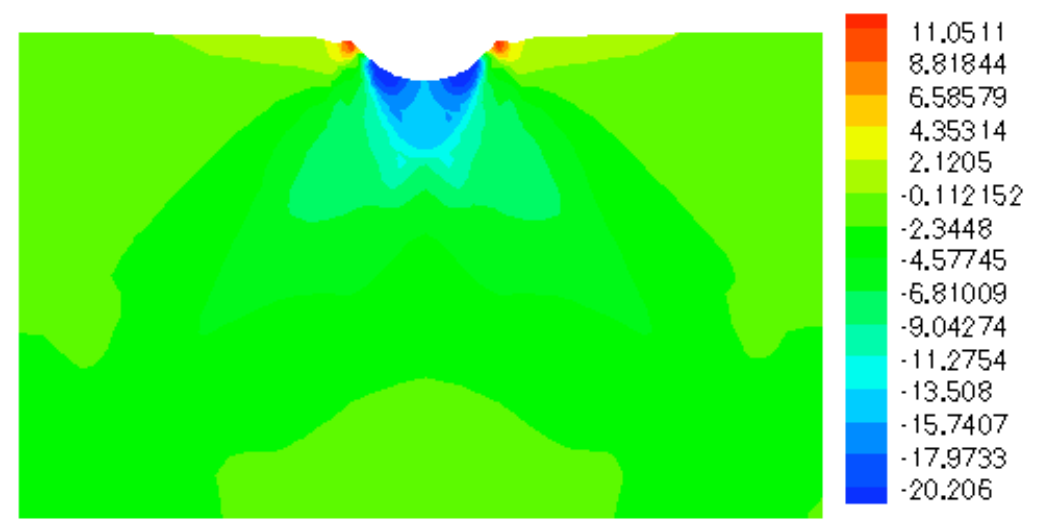

(c)

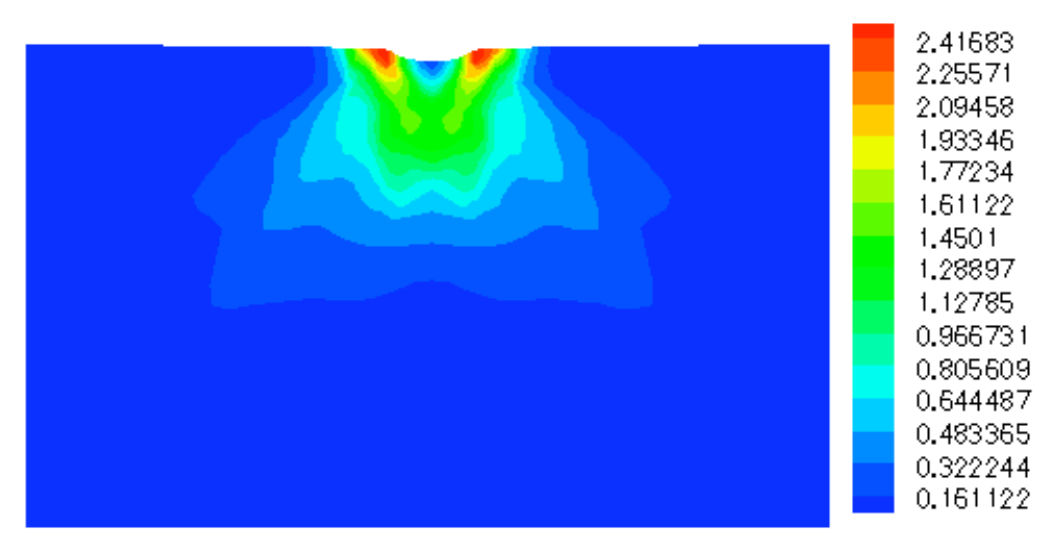

(b)

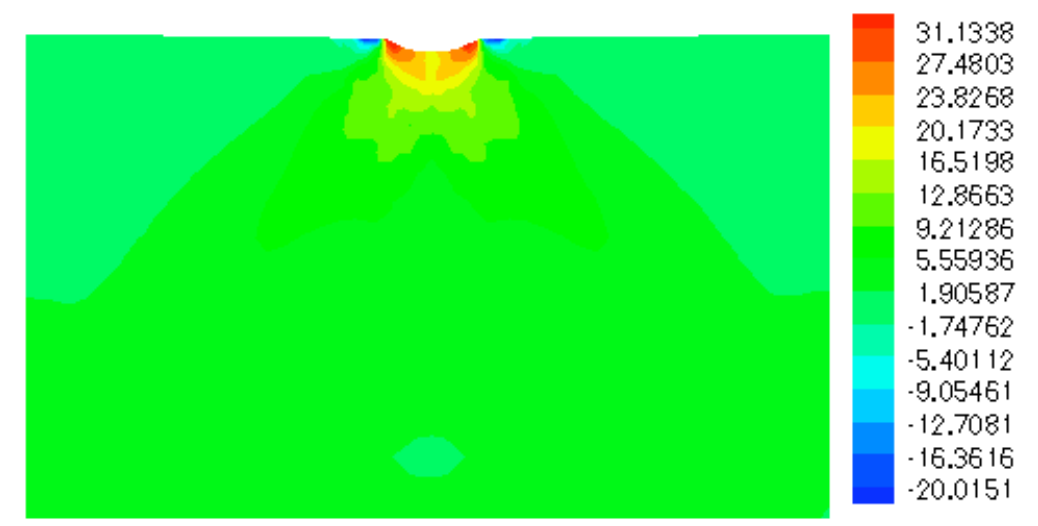

(d)

Figure 6.3. Plastic strain accumulation at final loading step (a), just before unloading step (b), pressure at final loading step normalized by yield stress (c), and pressure just before unloading step normalized by yield stress (d). (Model 1 with 9\% indentation) 


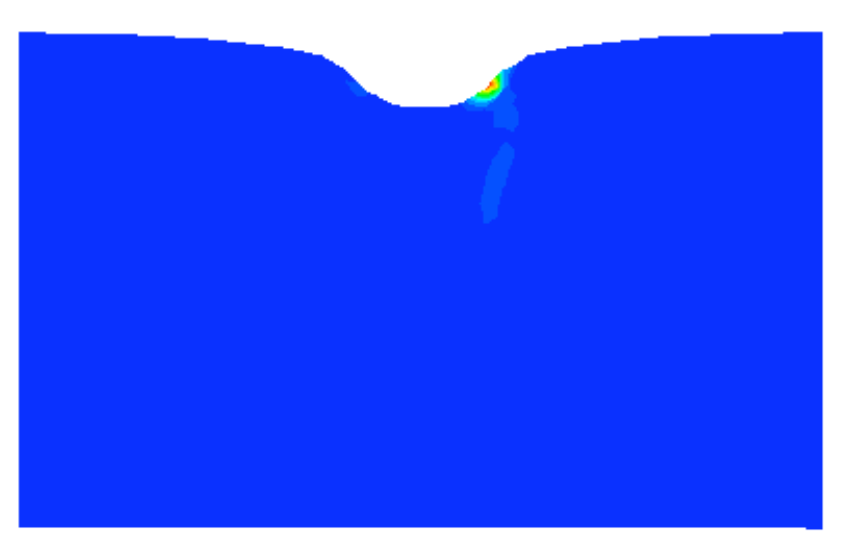

(a)

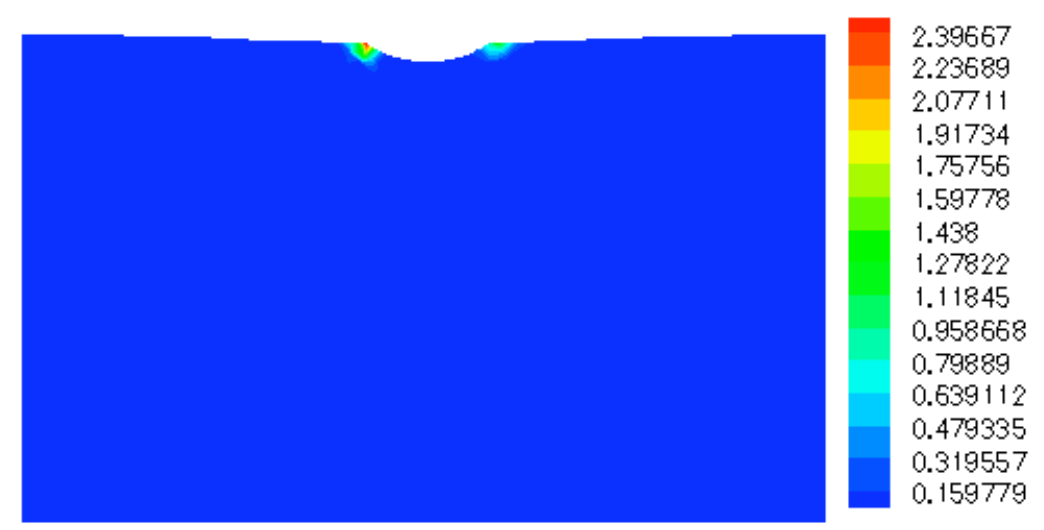

(c)

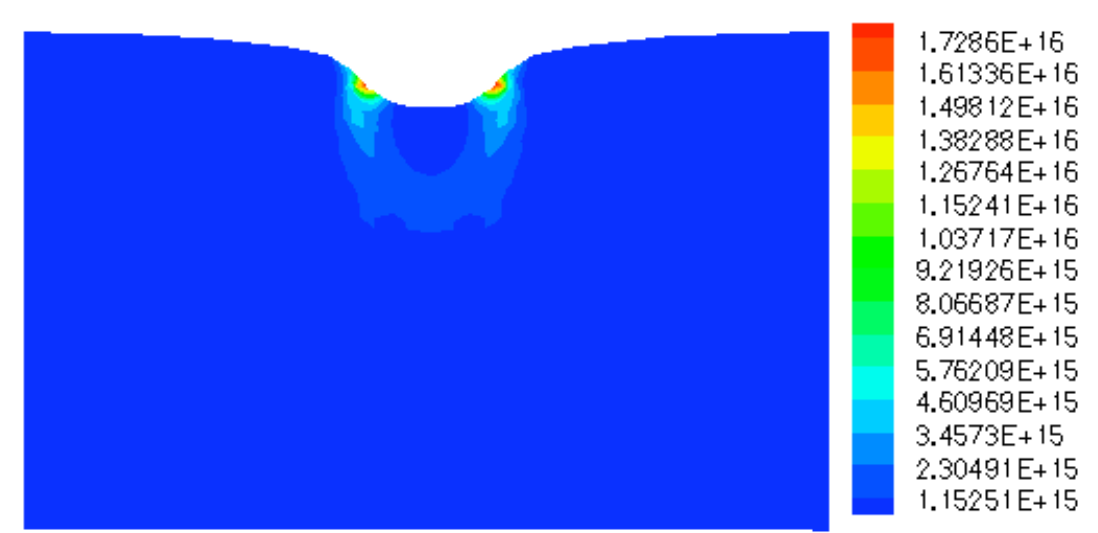

(b)

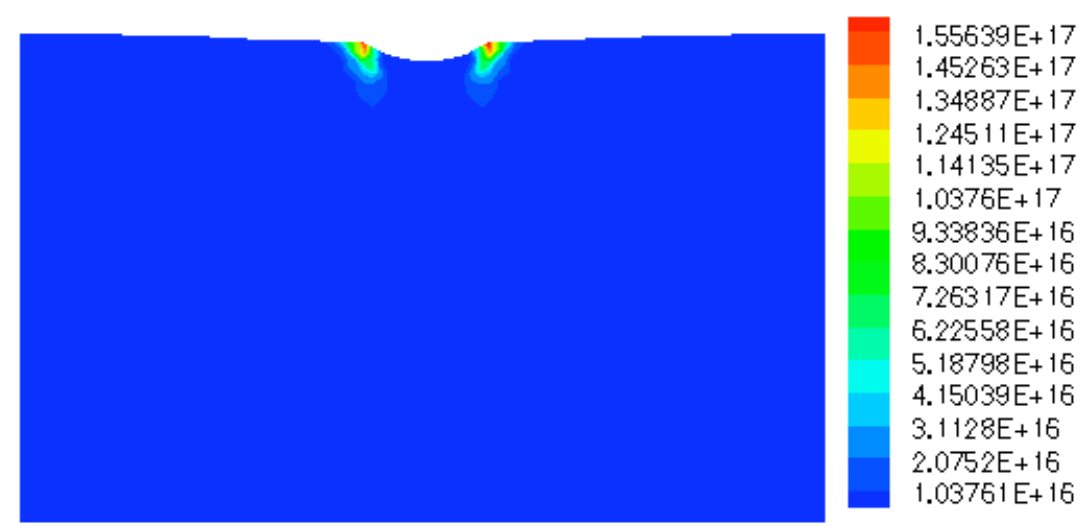

(d)

Figure 6.4. Immobile dislocation-density at final loading step for system 2 and 8 normalized by saturation (a), total immobile dislocation-density at final loading step non-normalized (b), just before unloading step for system 2 and 8 normalized by saturation (c), and total immobile dislocation-density just before unloading step non-normalized (d). (Model 1 with 12.8\% indentation) 


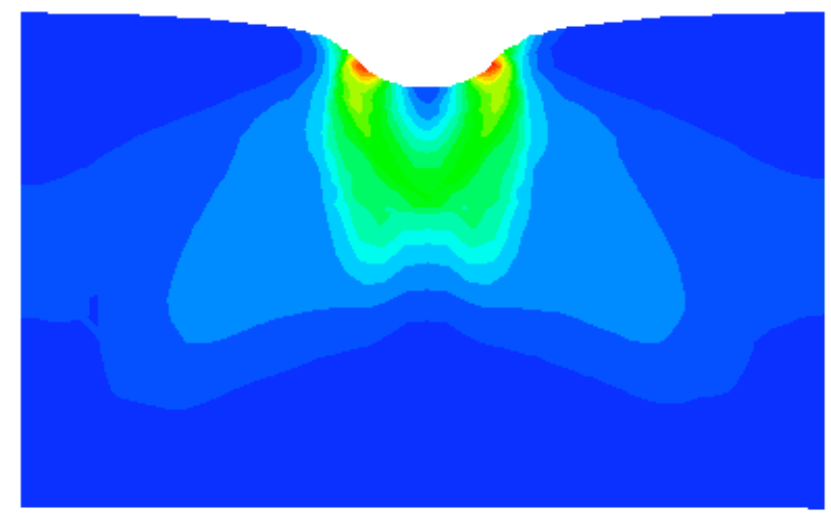

(a)

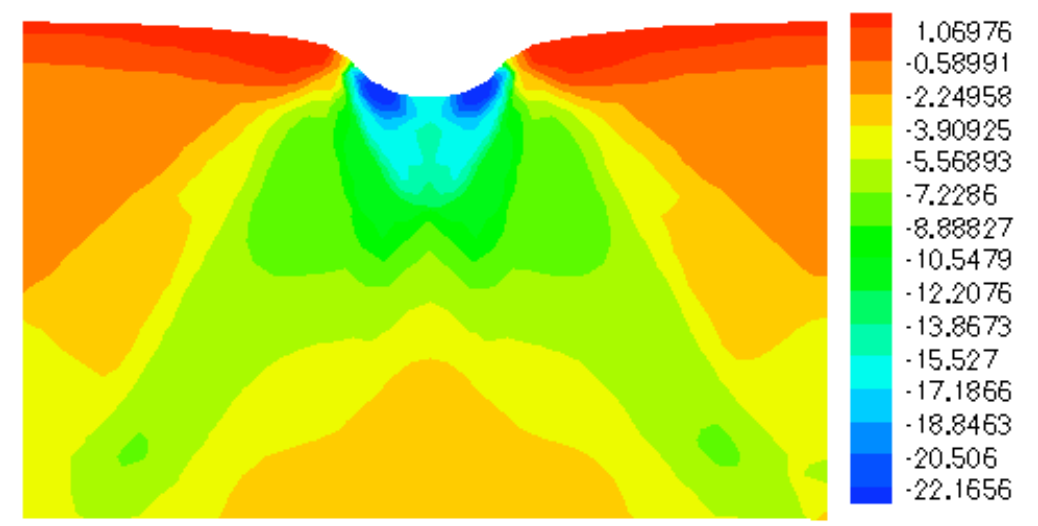

(c)

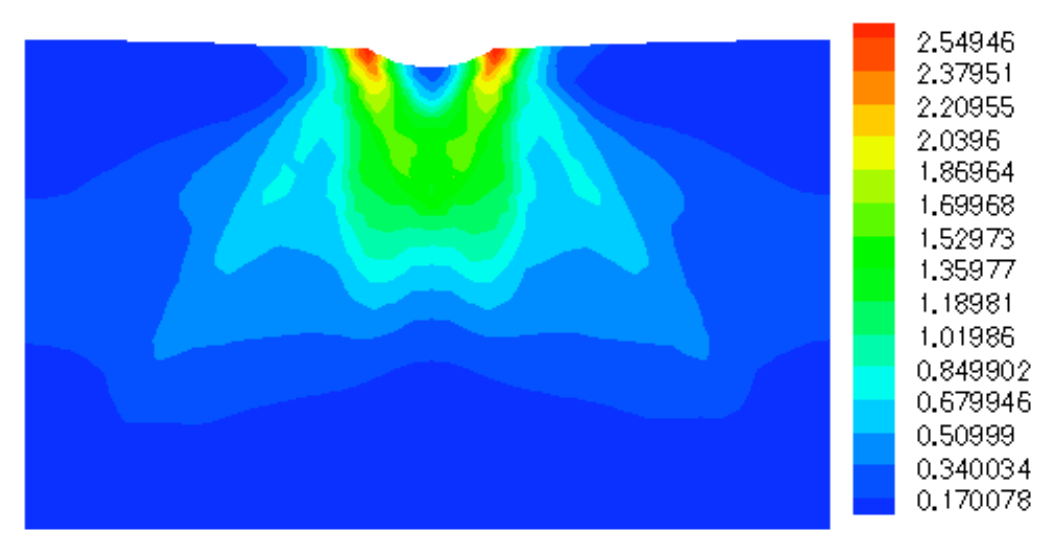

(b)

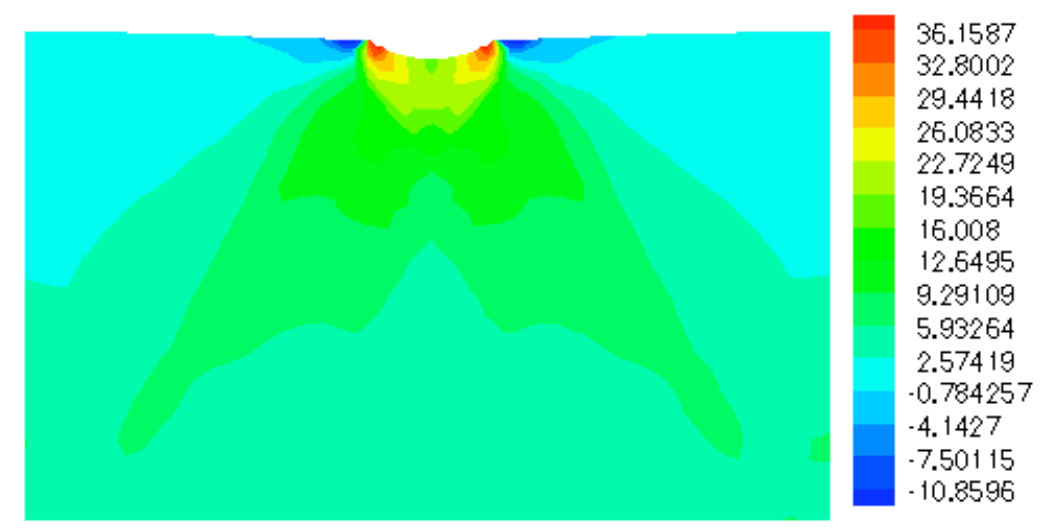

(d)

Figure 6.5. Plastic strain accumulation at final loading step (a), just before unloading step (b), pressure at final loading step normalized by yield stress (c), and pressure just before unloading step normalized by yield stress (d). (Model 1 with 12.8\% indentation) 


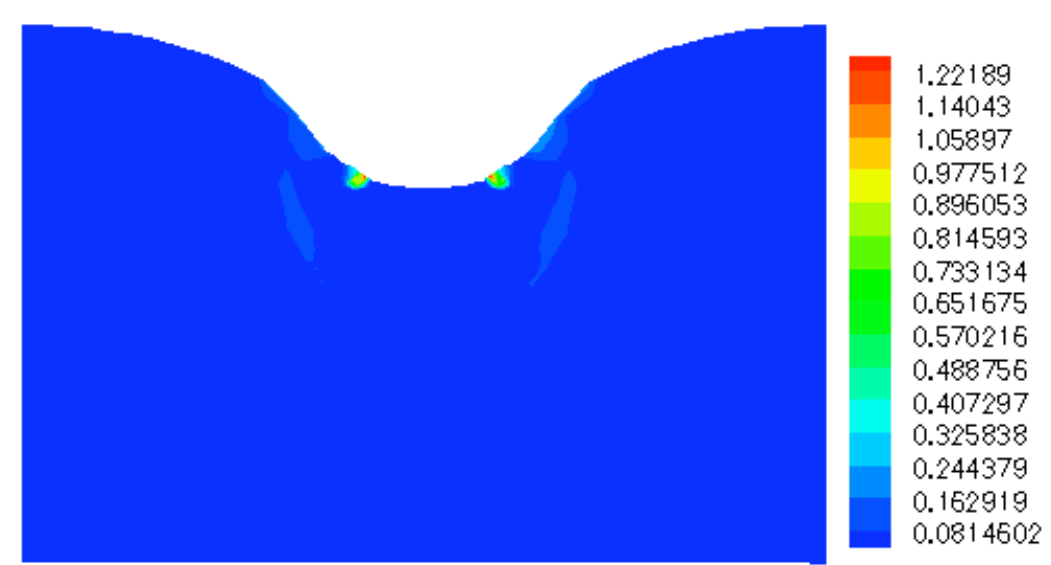

(a)

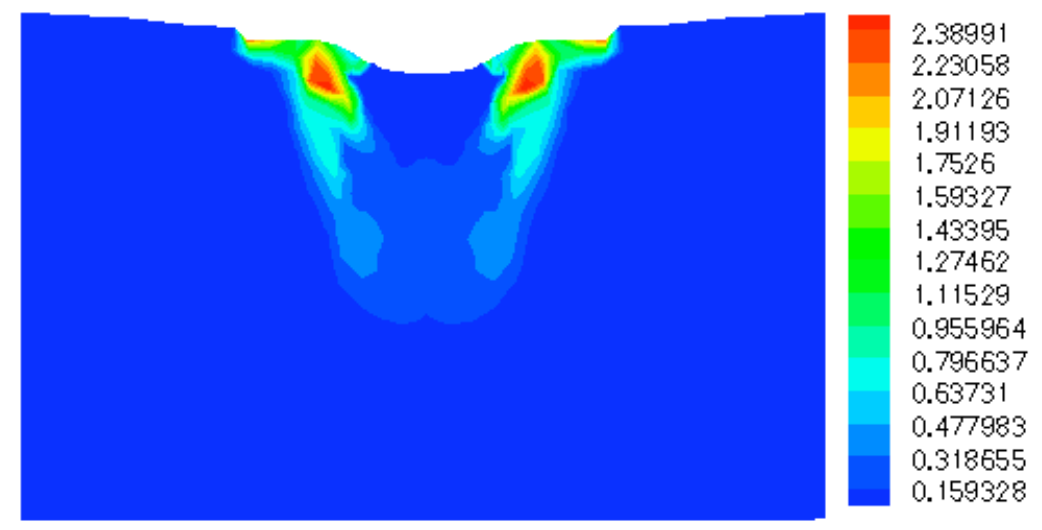

(c)

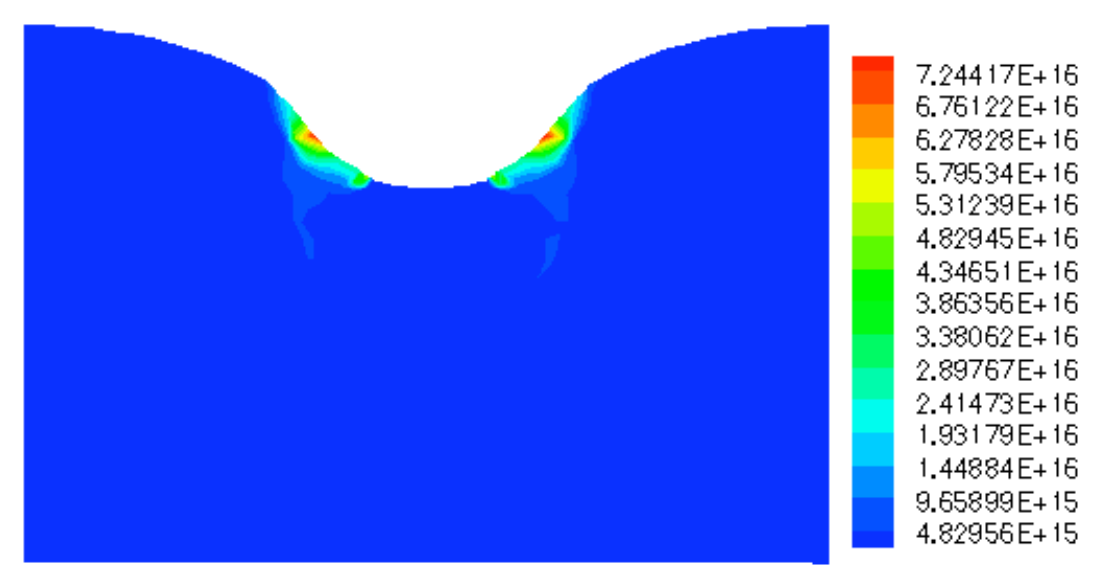

(b)

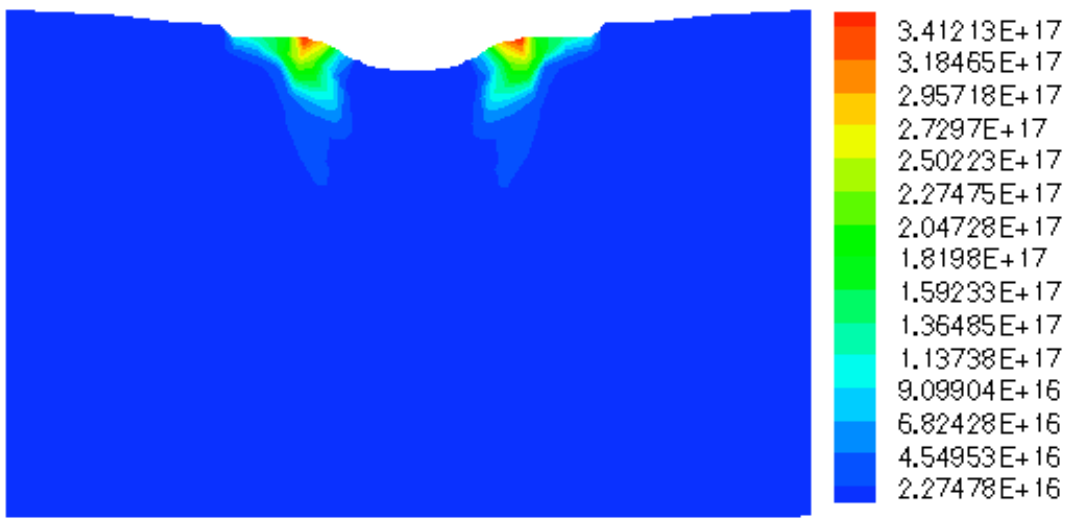

(d)

Figure 6.6. Immobile dislocation-density at final loading step for system 3, 4, 9, and 10 normalized by saturation (a), total immobile dislocation-density at final loading step non-normalized (b), just before unloading step for system 3 , 4, 9, and 10 normalized by saturation (c), and total immobile dislocation-density just before unloading step non-normalized (d). (Model 1 with $23.1 \%$ indentation) 


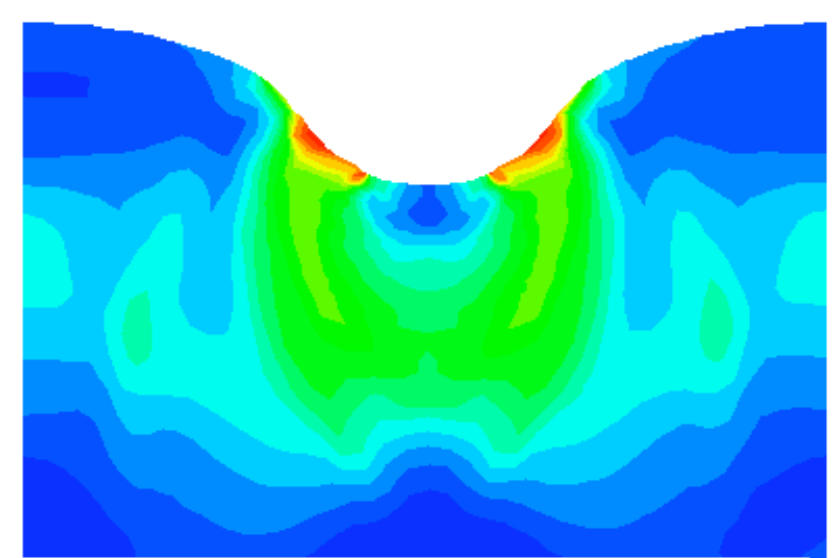

(a)

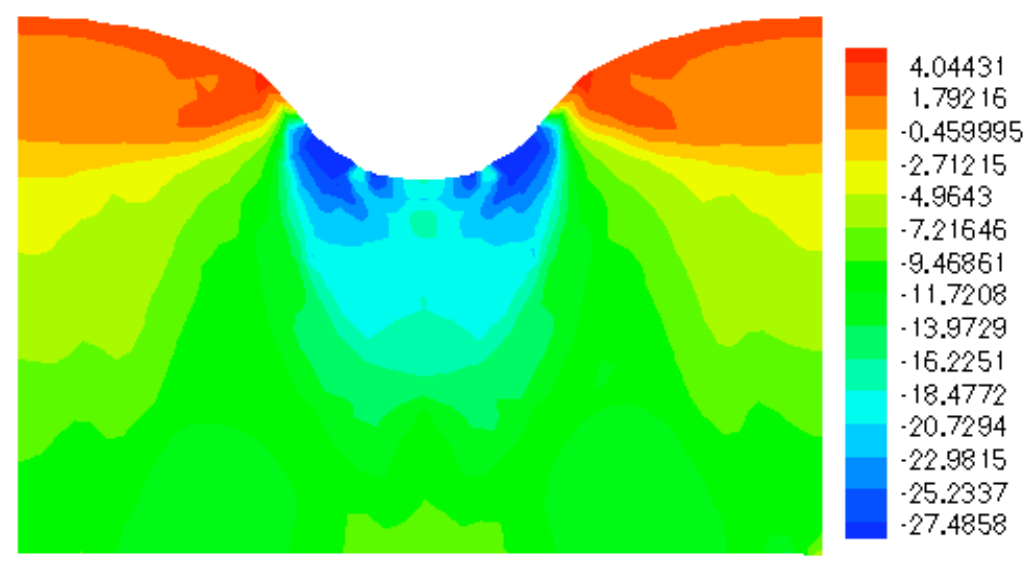

(c)

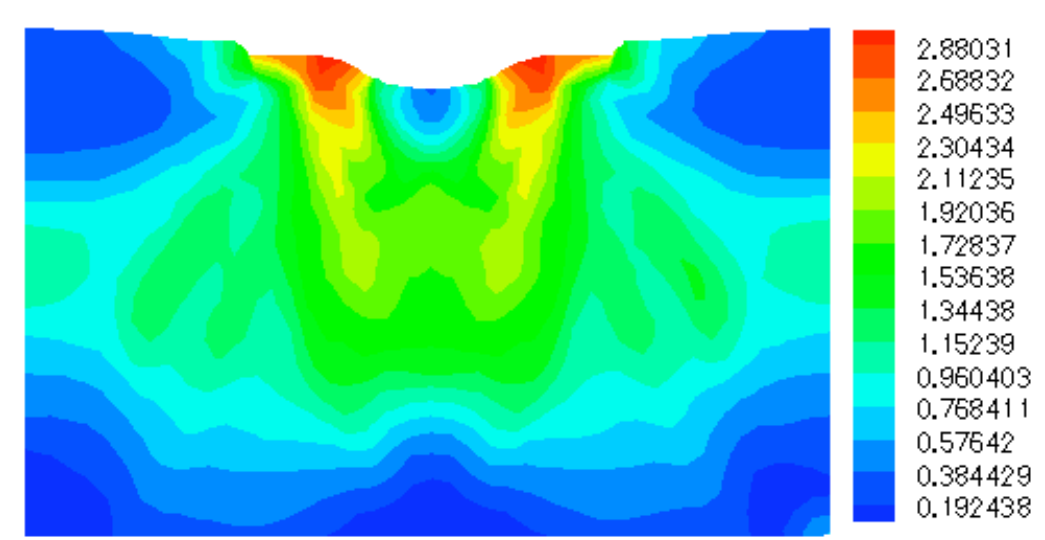

(b)

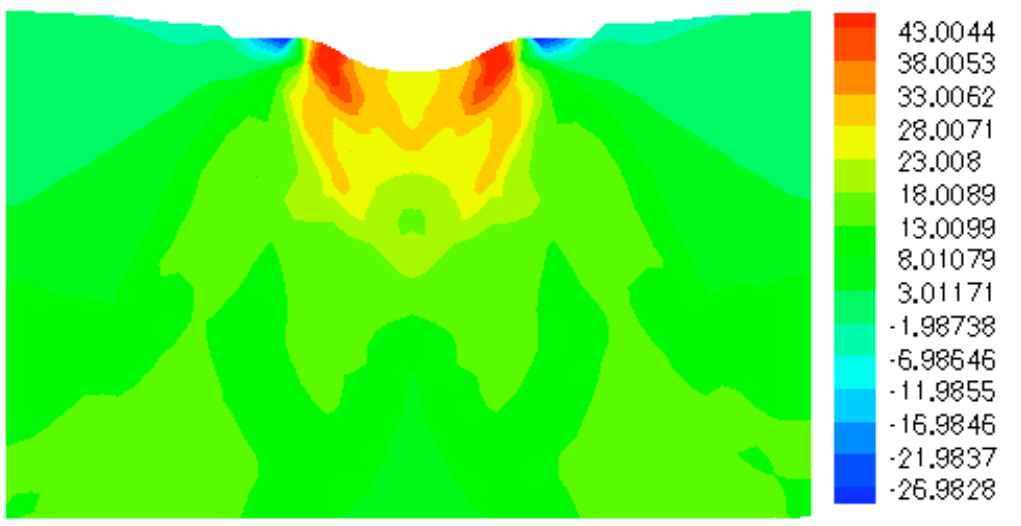

(d)

Figure 6.7. Plastic strain accumulation at final loading step (a), just before unloading step (b), pressure at final loading step normalized by yield stress (c), and pressure just before unloading step normalized by yield stress (d). (Model 1 with 23.1\% indentation) 


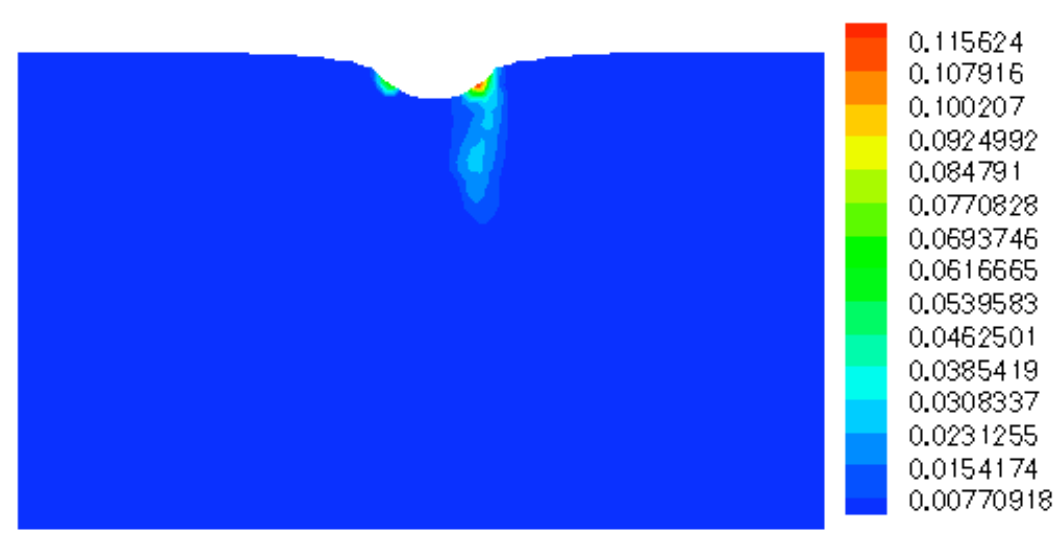

(a)

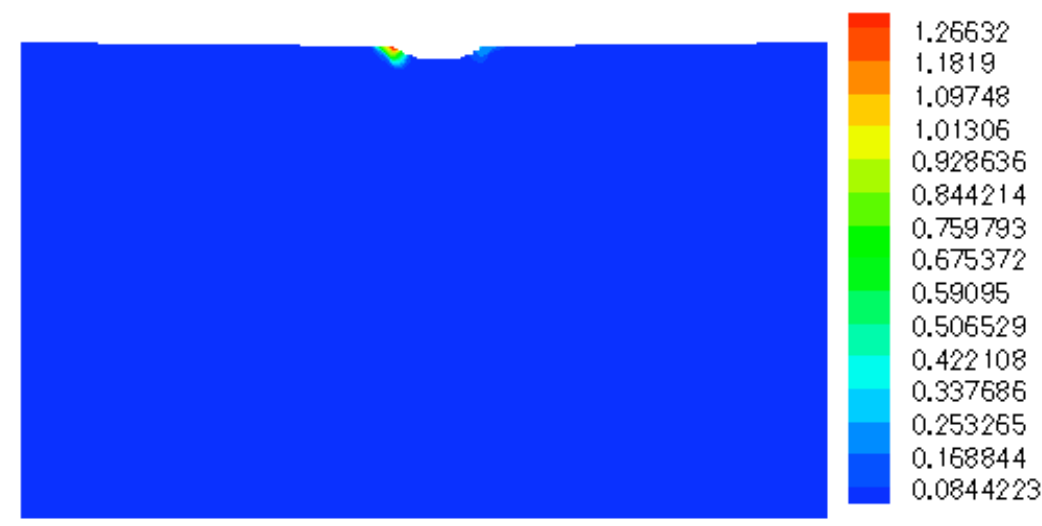

(c)

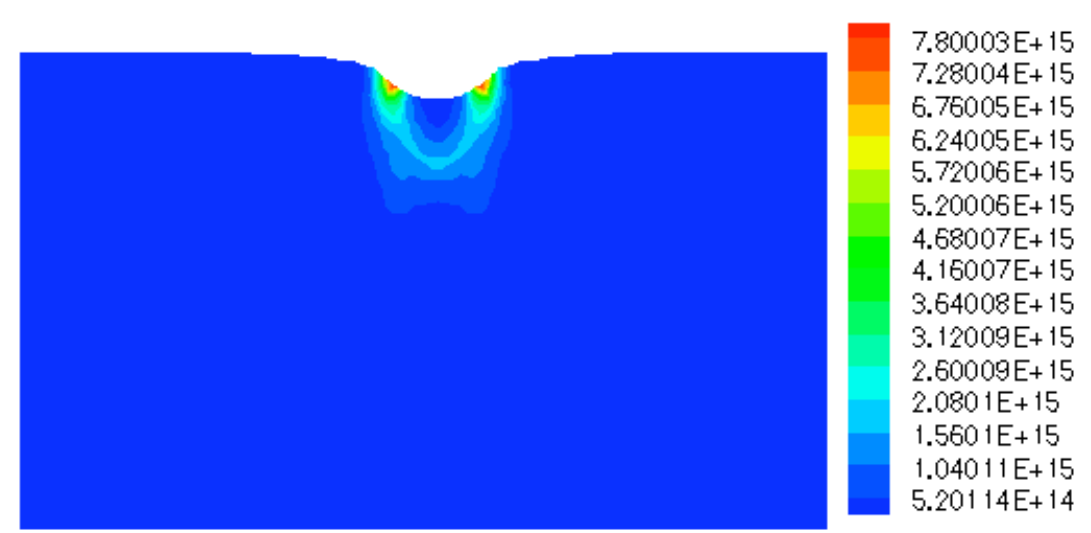

(b)

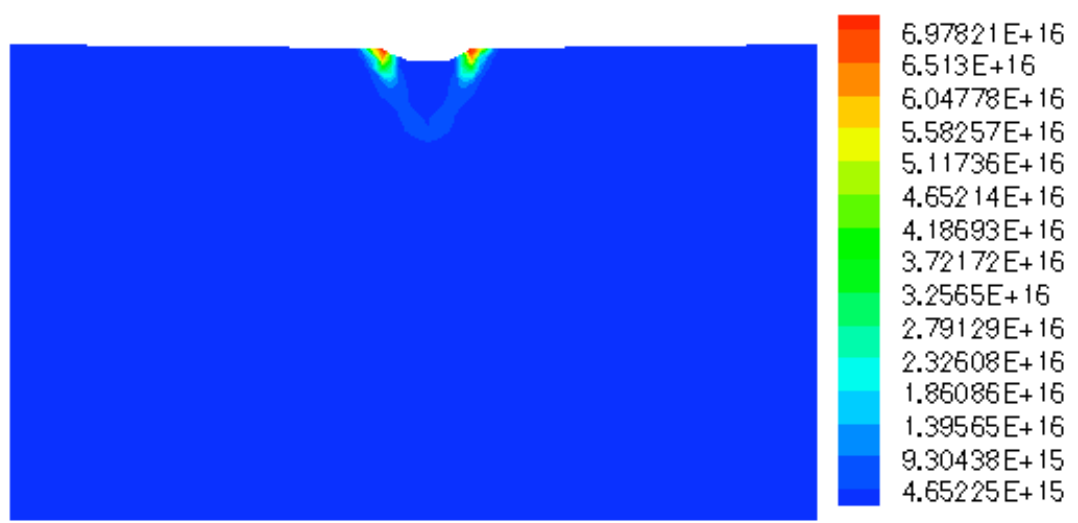

(d)

Figure 6.8. Immobile dislocation-density at final loading step for system 2 and 8 normalized by saturation (a), total immobile dislocation-density at final loading step non-normalized (b), just before unloading step for system 2 and 8 normalized by saturation (c), and total immobile dislocation-density just before unloading step non-normalized (d). (Model 2 with 9\% indentation) 


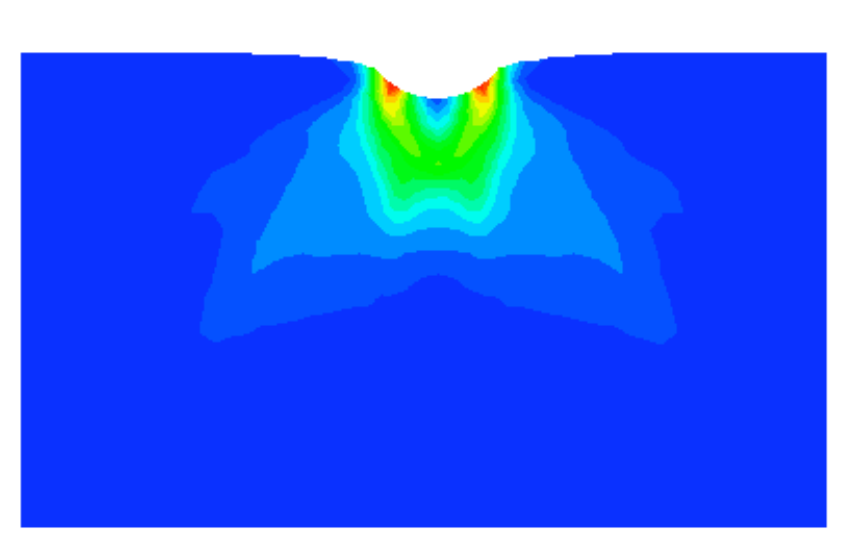

(a)

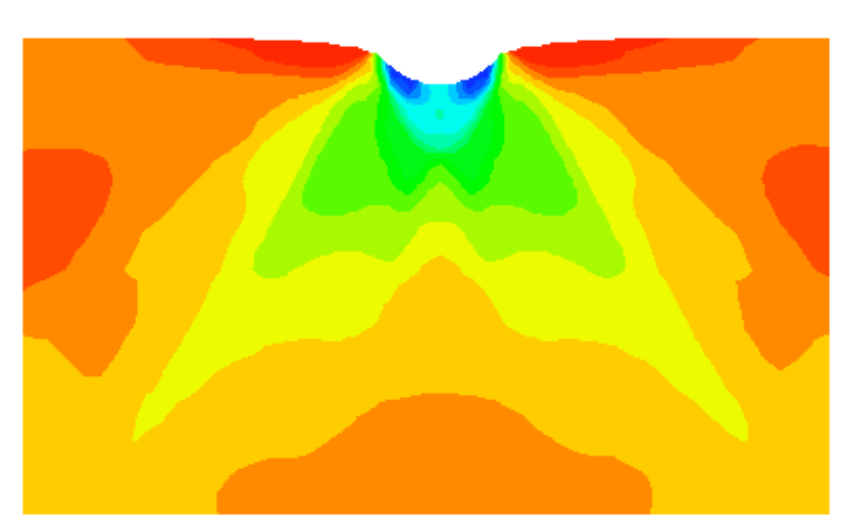

(c)

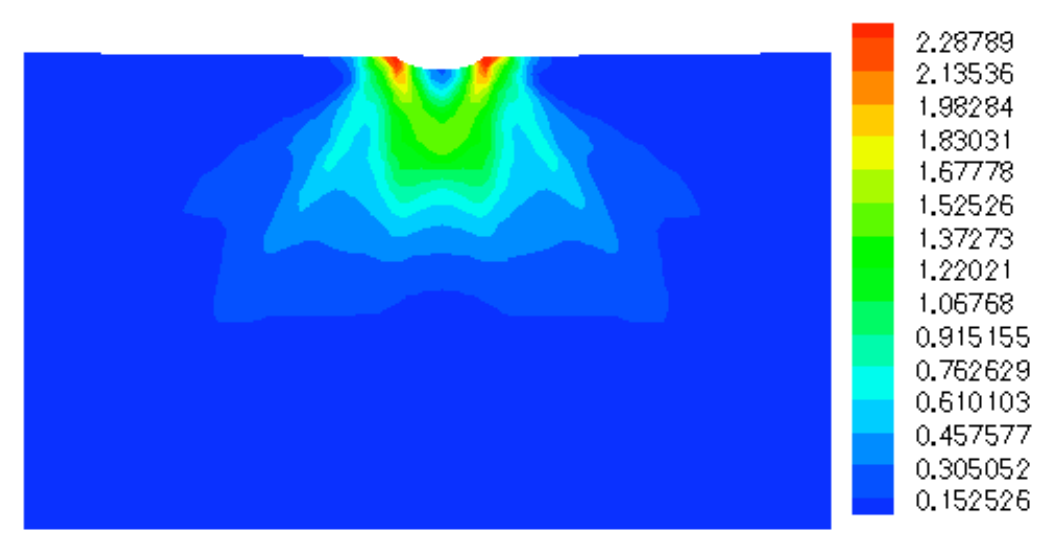

(b)

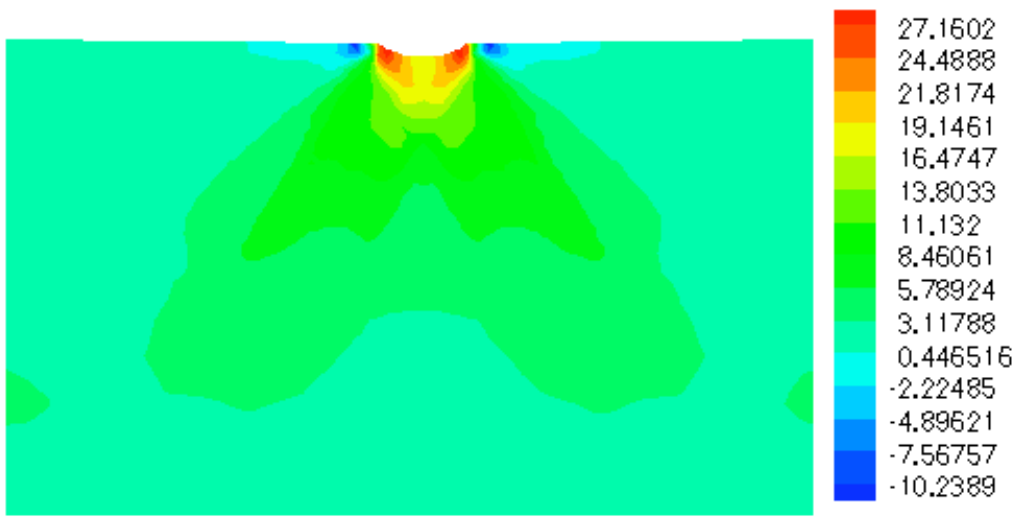

(d)

Figure 6.9. Plastic strain accumulation at final loading step (a), just before unloading step (b), pressure at final loading step normalized by yield stress (c), and pressure just before unloading step normalized by yield stress (d). (Model 2 with 9\% indentation) 


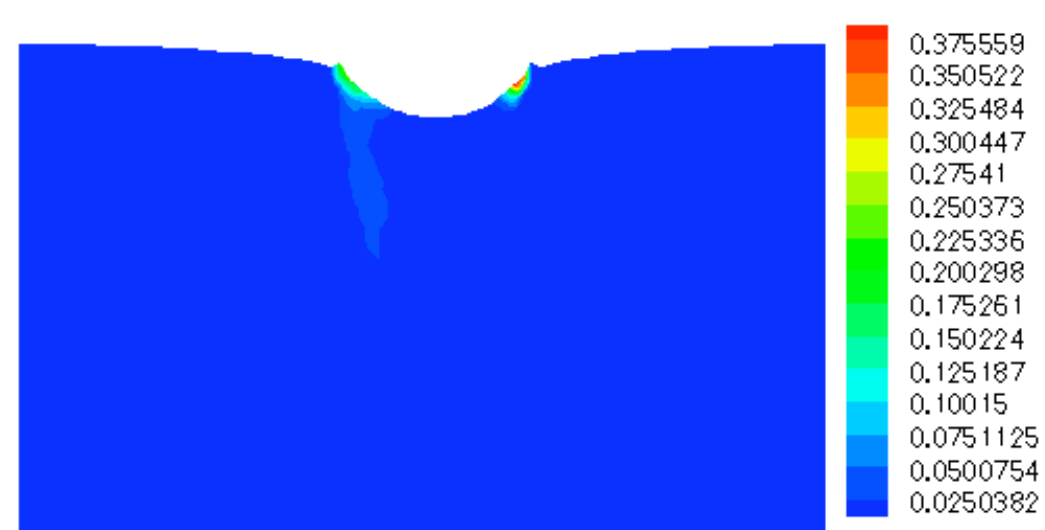

(a)

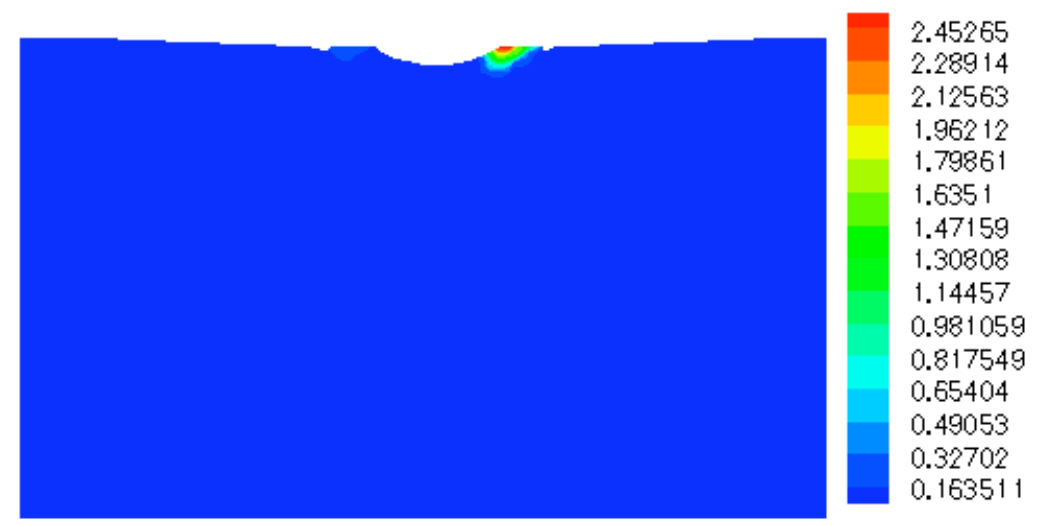

(c)

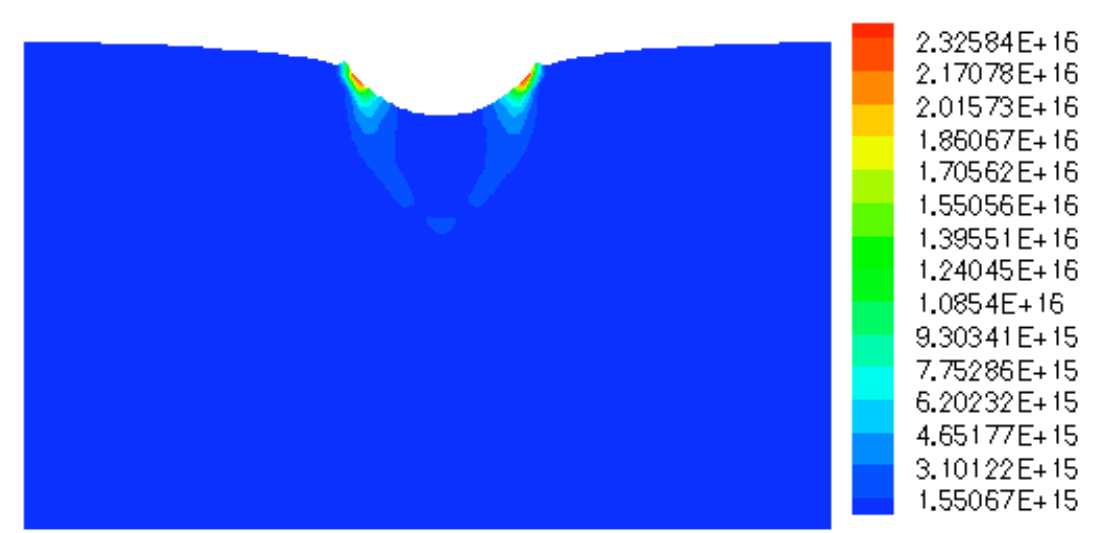

(b)

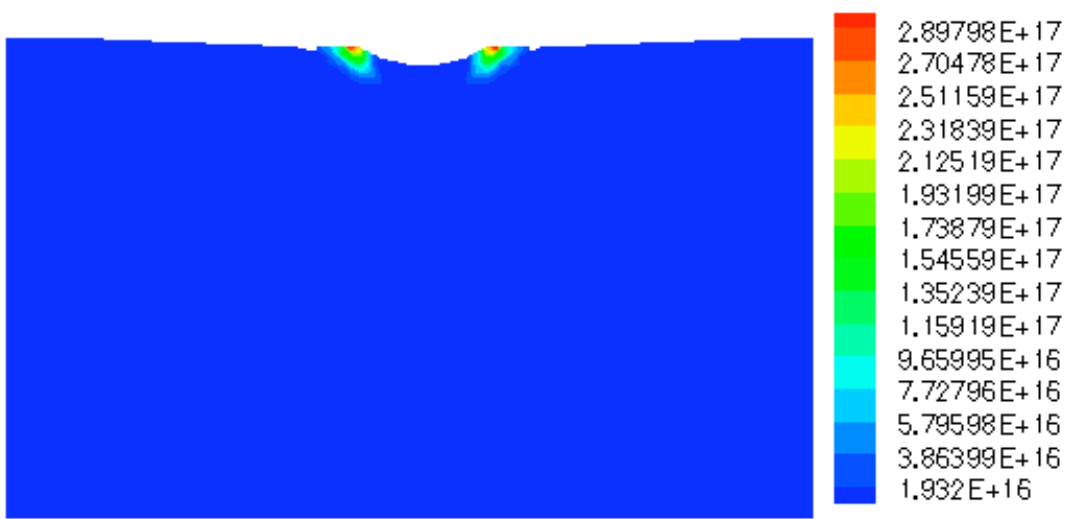

(d)

Figure 6.10. Immobile dislocation-density at final loading step for system 1 and 7 normalized by saturation (a), total immobile dislocation-density at final loading step non-normalized (b), just before unloading step for system 1 and 7 normalized by saturation (c), and total immobile dislocation-density just before unloading step non-normalized (d). (Model 2 with 12.8\% indentation) 


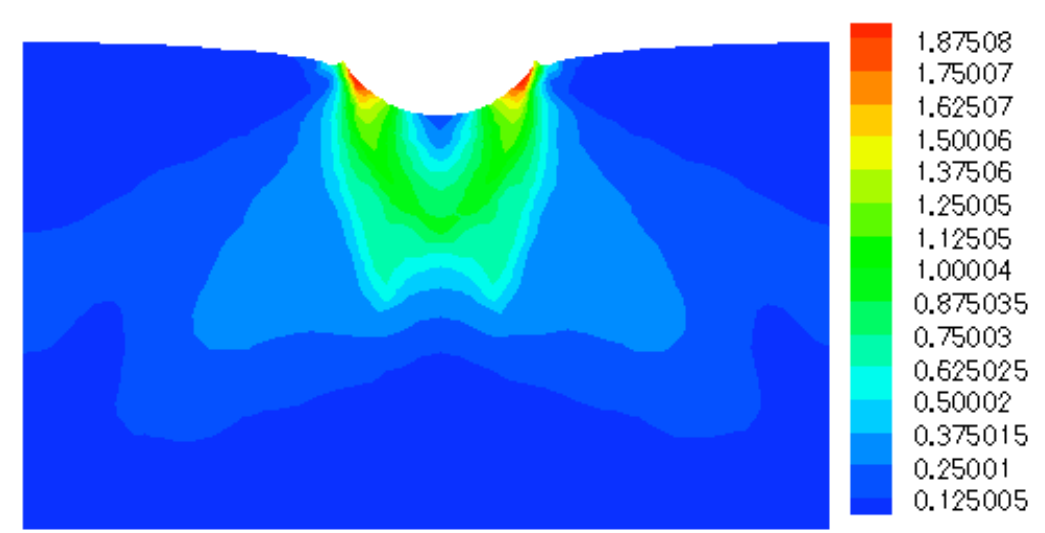

(a)

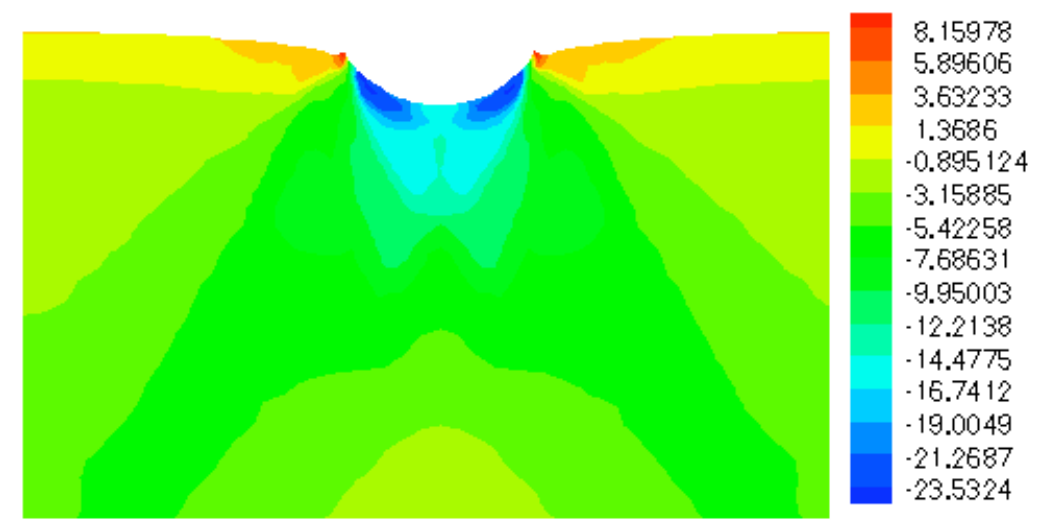

(c)

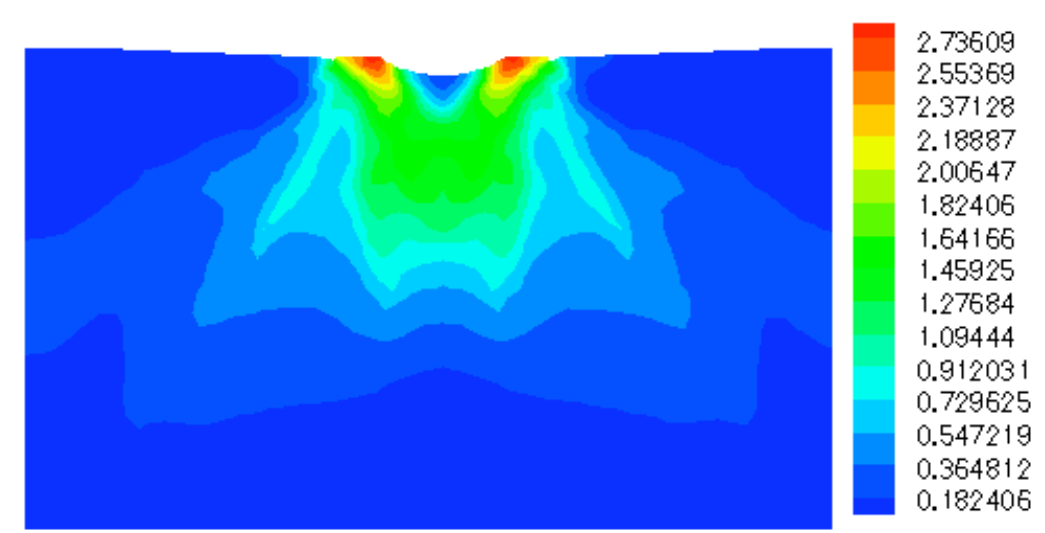

(b)

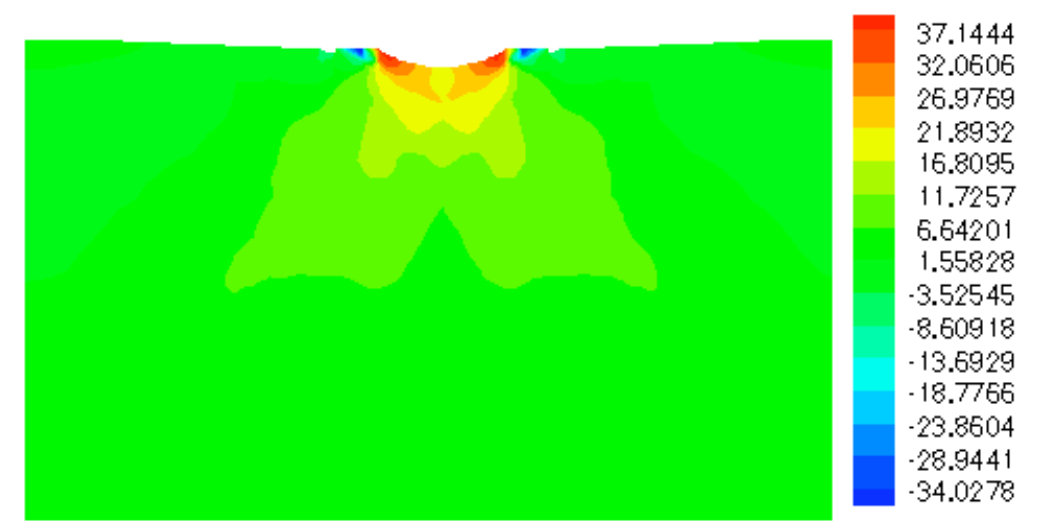

(d)

Figure 6.11. Plastic strain accumulation at final loading step (a), just before unloading step (b), pressure at final loading step normalized by yield stress (c), and pressure just before unloading step normalized by yield stress (d). (Model 2 with $12.8 \%$ indentation) 


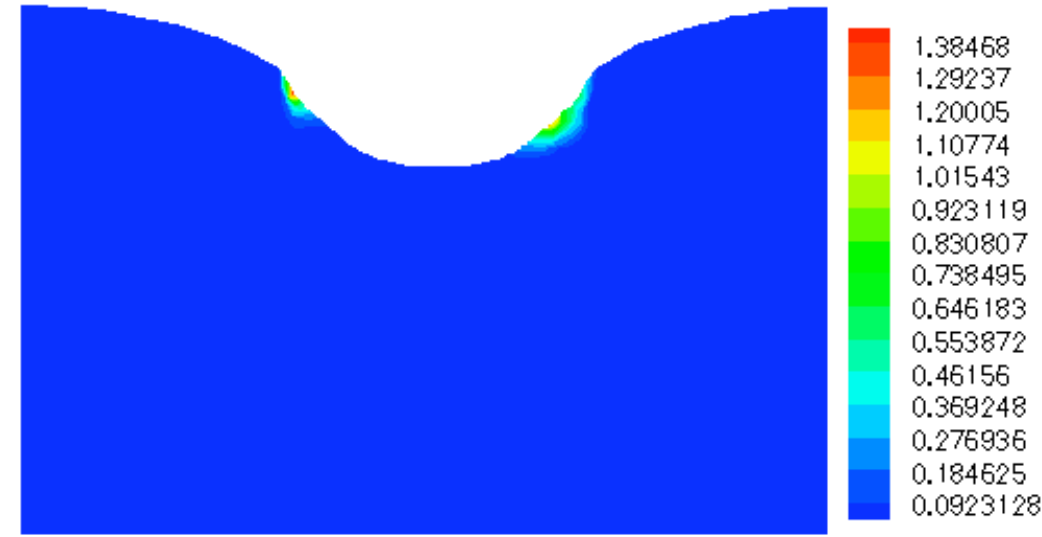

(a)

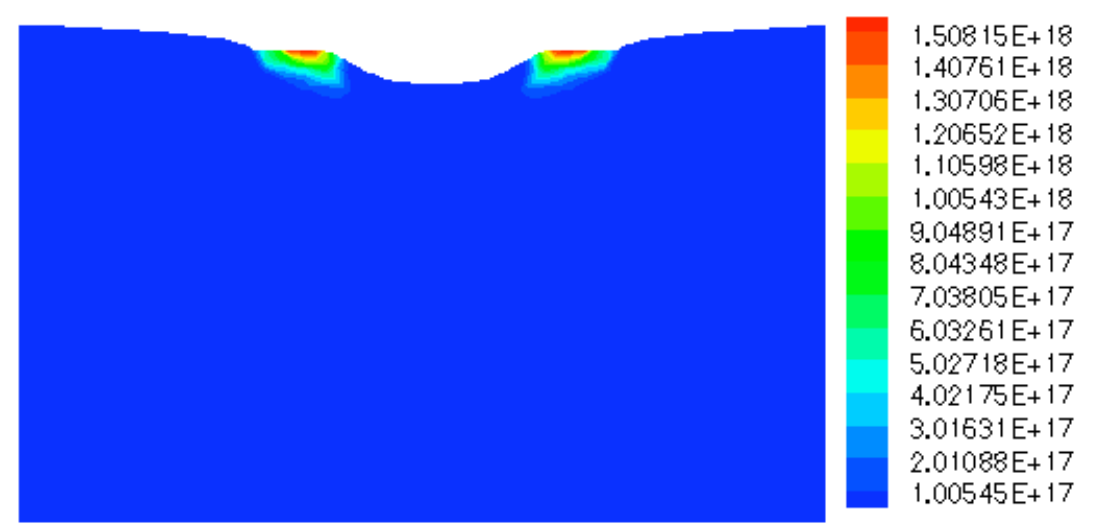

(c)

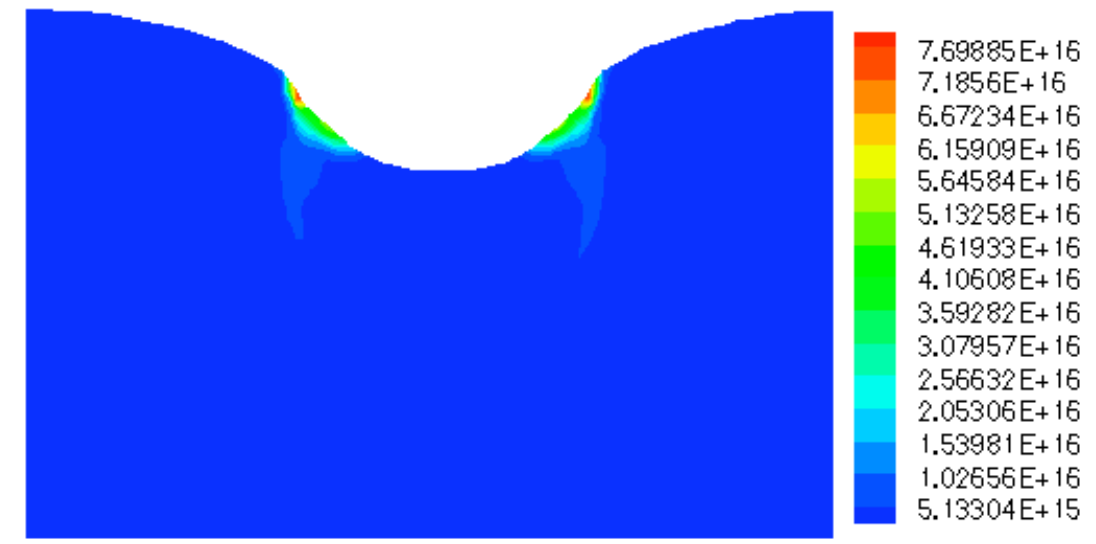

(b)

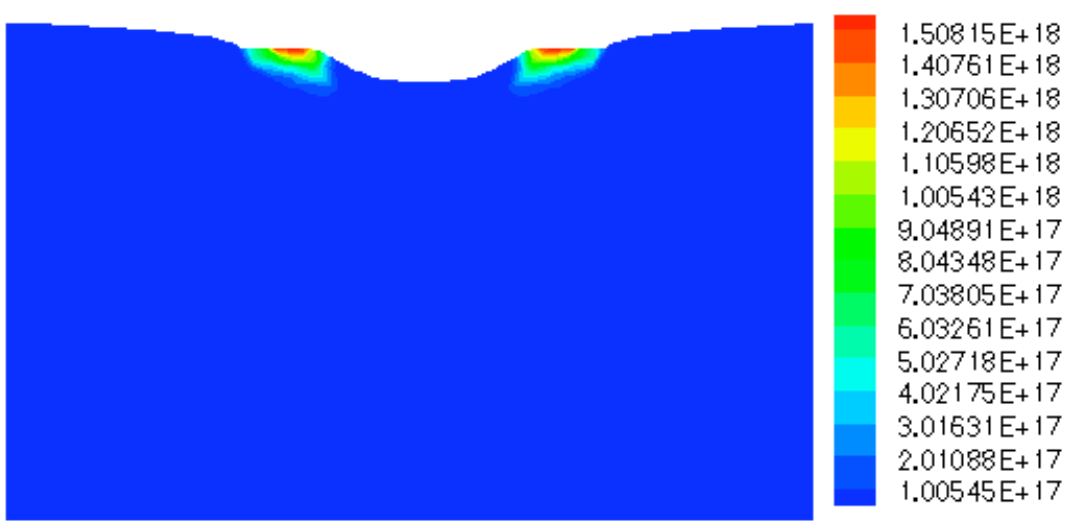

(d)

Figure 6.12. Immobile dislocation-density at final loading step for system 6 and 12 normalized by saturation (a), total immobile dislocation-density at final loading step non-normalized (b), just before unloading step for system 6 and 12 normalized by saturation (c), and total immobile dislocation-density just before unloading step non-normalized (d). (Model 2 with 23.1\% indentation) 


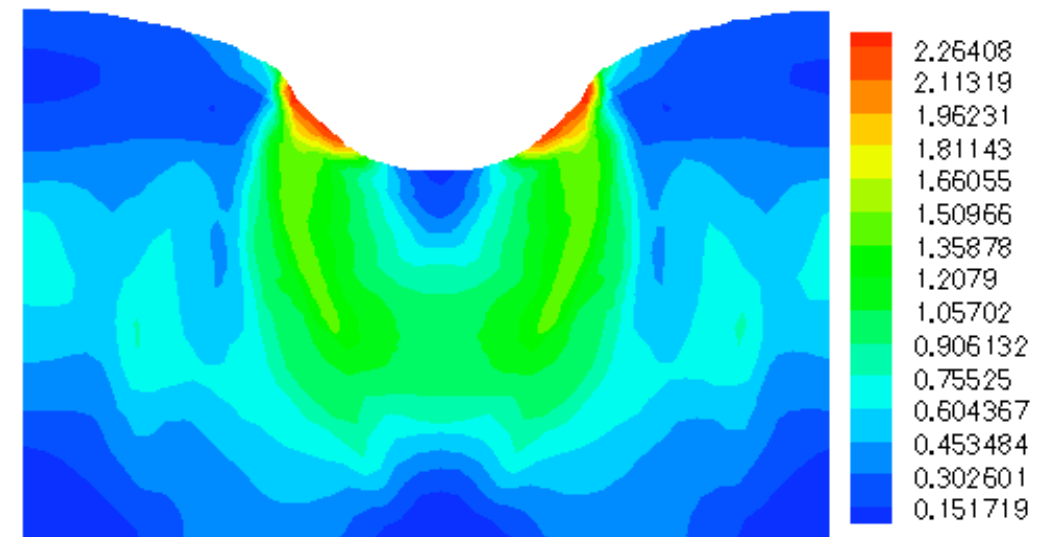

(a)

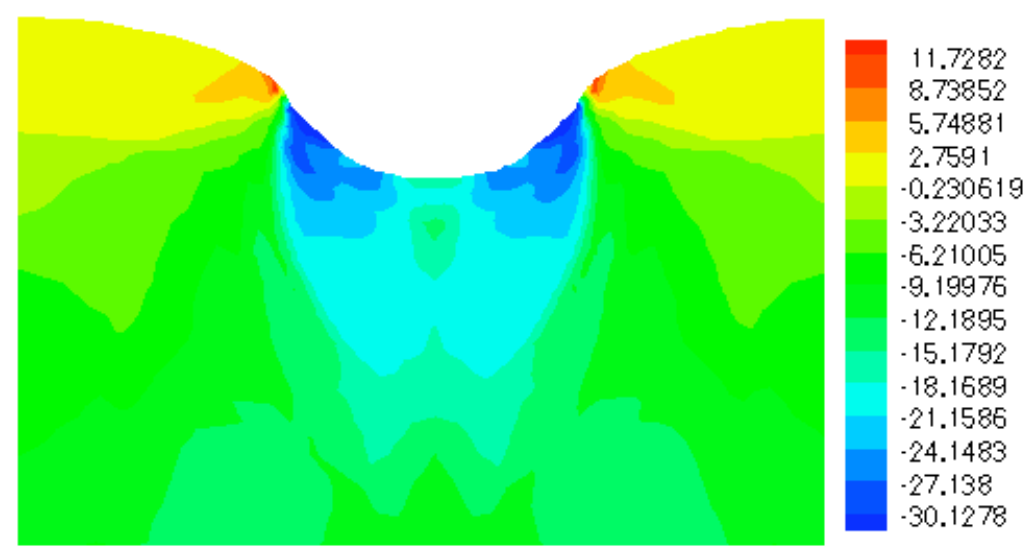

(c)

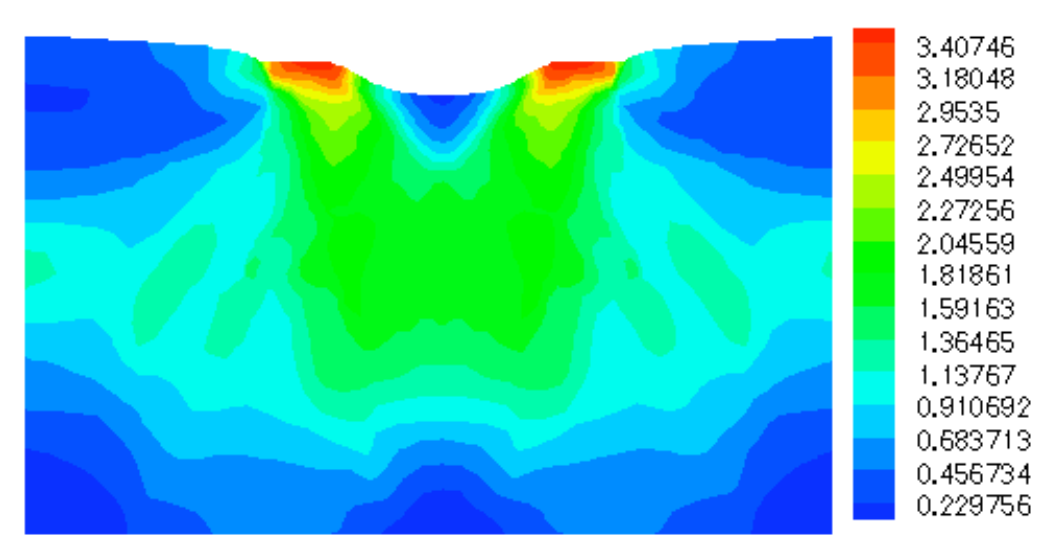

(b)

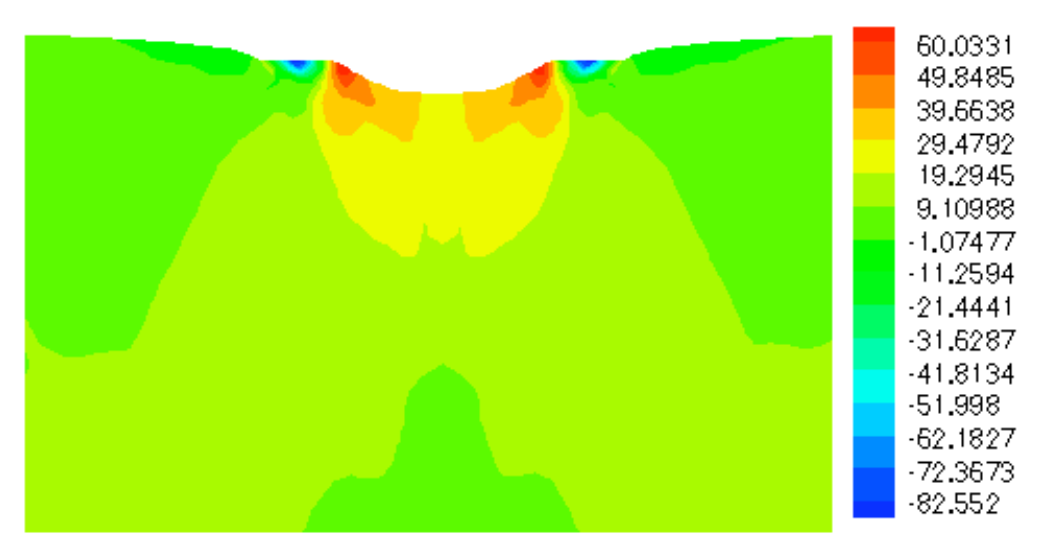

(d)

Figure 6.13. Plastic strain accumulation at final loading step (a), just before unloading step (b), pressure at final loading step normalized by yield stress (c), and pressure just before unloading step normalized by yield stress (d). (Model 2 with $23.1 \%$ indentation) 


\section{CHAPTER 7}

\section{NANOINDENTATION OF POLYCRYSTALLINE AGGREGATES WITH RANDOM LOW ANGLE GB MISORIENTATIONS}

In this chapter, we apply the hierarchical computational model, the dislocationdensity based crystalline formulation, and the GB interfacial kinematic scheme to investigate the effects of varying the Euler angles within an aggregate to represent GB misorientations, which are representative of low angle GB misorietations, where the misorientations do not exceed $15^{\circ}$ (see, for example, Randle, 1993). Hence, we will be able to characterize and predict how nanoindentation is affected by GB effects, such as dislocation-density impedances and transmissions between and within grains. More importantly, we will be able to better understand how GBs affect and possibly control inelastic deformation modes during nanoindentation on a scale that spans the nano to the macro.

\subsection{Modeling of Polycrystalline Aggregates with Low Angle GBs}

Two polycrystalline aggregates with grain-sizes of $0.0022 \mathrm{~mm}$ and $0.003 \mathrm{~mm}$ were investigated. It was assumed that the GB region is one-tenth of the grain diameter for both these grain-sizes. In this formulation, the mesh area corresponding to the GB region is modeled as a distinct region from the grain interior, and hence the dislocation-density evolution and properties can be varied within the GB. All regions were randomly misoriented by Euler angles, $\varphi_{1}, \Phi$, and $\varphi_{2}$ (Figure 7.1), and its maximum misorientation angle is constrained by fifteen degrees, such that it is representative of low angle GB distribution. The same loading rates and properties that were used in chapter 6 are used here. The schematic 
for the nanoindentation model of the polycrystalline aggregates with the distinct GBs is shown in Figure 7.1. In the interfacial GB regions, the initial mobile and immobile dislocation-densities were varied as a function of the random GB misorientation as

$$
\begin{aligned}
& \rho_{i m o}^{G B}=\rho_{i m o}^{G} \times \kappa, \\
& \rho_{m o}^{G B}=\rho_{m o}^{G} / \kappa,
\end{aligned}
$$

where $\kappa$ is $1.58^{\theta}, \theta$ is the maximum misorientation angle, $\rho_{\text {imo }}^{G B}$ is the interfacial GB initial immobile dislocation-density, $\boldsymbol{\rho}_{m o}^{G B}$ is the interfacial GB initial mobile dislocation-density , $\rho_{\text {imo }}^{G}$ is the bulk initial immobile dislocation-density , and $\boldsymbol{\rho}_{m o}^{G}$ is the bulk initial mobile dislocation-density. The initial immobile dislocation-density values for bulk grain, $\rho_{i m o}^{G}$ and $\boldsymbol{\rho}_{m o}^{G}$, was given in chapter 5 . And the initial immobile dislocation-density values for GBs, $\boldsymbol{\rho}_{\text {imo }}^{G B}$ and $\boldsymbol{\rho}_{m o}^{G B}$, vary $10^{10} \sim 10^{12} \mathrm{~m}^{-2}$ for immobile and $10^{5} \sim 10^{7} \mathrm{~m}^{-2}$ for mobile (Ashmawi and Zikry, 2000).

The following assumptions were also used:

- The initial GB static yield stress and modulus were assumed to be the same as the bulk grain;

- The GB dislocations are of the same type as the bulk lattice.

The grain bulk and GB properties are same as in Table 5.1. The larger-sized grain dimension was modeled with an aggregate size of fifteen grains (Model 1). Since, we are also using the previous specimen dimensions, the aggregate size for the smaller grain-sizes is therefore twenty-eight grains (Model 2). 


\subsection{Grain-size Effects and Slip-system Activities during Local Unloading}

The polycrystalline aggregate with the random low angle grain orientations and the two grain-sizes of $0.003 \mathrm{~mm}$ (Model 1, 15 grains) and $0.0022 \mathrm{~mm}$ (Model 2, 28 grains) were investigated. The global stress-strain curves are shown in Figure 7.2. As seen from these figures, as the compressive nominal strains increases, the average stress increases for both Models 1 and 2. During unloading, the smaller grain-sized aggregate has large nominal strains. As noted earlier, these residual strains are directly related to that more grains are indented, as the grain-sizes are reduced, which results in more slip-system activation, and consequently leads to greater inelastic activity.

As we analyzed previously, we investigated slip-system activation during the local unloading of the stress-strain curve during the indentation process. In the cube-oriented aggregate analysis, there were several slip-system activities before and after local unloading points (as indicated in Figure 6.1). For the large-sized grains, Model 1, at a normalized depth of $9 \%$ and $12.8 \%$, slip-system 9 was most active before each of these local load-drops, and slip-system 5 was most activated after the drop. However, at a normalized depth of $23.1 \%$, slip-system 6 was most active before and after the load-drop (a summary of these activities is given in Table 7.1). For the smaller grain-sized aggregate (Model 2), there was a slip-system switch at the three indented depths (Table 7.1). The different slip-systems and immobile dislocation-densities are activated due to lattice rotations and accumulations of plastic strains and pressures in indented regions and grains. These phenomena have experimentally observed in the nanoindentation of crystalline materials (see, for example, Michalske and 
Houston, 1998), but what is significant is that in our computational analysis, we can capture these events without imposing any a priori assumptions related to slip-system activation.

Table 7.1. Slip-system activity variation before and after local load-drops during global loading (low angle GB misorientation)

\begin{tabular}{ccc}
\hline Normalized Indented Depth & Before load-drop & After load-drop \\
\hline $9 \%$ Model 1 & 9 & 5 \\
12.8\% Model 1 & 9 & 5 \\
23.1\% Model 1 & 6 & 6 \\
$9 \%$ Model 2 & 9 & 3 \\
12.8\% Model 2 & 3 & 8 \\
23.1\% Model 2 & 1 & 2 \\
\hline
\end{tabular}

\subsection{Immobile Dislocation-density Evolution, Plastic Strain, and Pressure Response: Model 1}

We tracked the most active slip-systems at different normalized depths to determine how the immobile dislocation-densities on these systems evolve. A summary is given in Table 7.2.

Table 7.2. List of most active slip-systems at maximum normalized depths

\begin{tabular}{cccc}
\hline $\begin{array}{c}\text { Normalized } \\
\text { Indented Depth }\end{array}$ & Most active slip-systems & Normalized Depth & Most active slip-systems \\
\hline $9 \%$ Model 1 & 5 & $9 \%$ Model 2 & 3 \\
12.8\% Model 1 & 5 & $12.8 \%$ Model 2 & 1 \\
$23.1 \%$ Model 1 & 12 & $23.1 \%$ Model 2 & 2 \\
\hline
\end{tabular}

We also investigated material behavior at the final global indented loading and unloading points. At a normalized depth of $9 \%$, the immobile dislocation-density on slipsystem 5, at the maximum loading step, had evolved to $2.08 \times 10^{16} \mathrm{~m}^{-2}$ (Figure $7.3 \mathrm{a}$ ). The total immobile dislocation-density for all slip-systems at this depth is $2.42 \times 10^{16} \mathrm{~m}^{-2}$ (Figure $7.3 \mathrm{~b}$ ). This indicates a strong dominance by this single system. At global unloading, at this 
normalized depth, for slip-system 5 , the immobile dislocation-density is $5.07 \times 10^{16} \mathrm{~m}^{-2}$ (Figure 7.3c), and the total immobile dislocation-density is $8.43 \times 10^{16} \mathrm{~m}^{-2}$ (Figure 7.3d). Hence, before unloading the immobile dislocation-density of the most active slip-system accounts for $85 \%$ of all immobile dislocation-density activities. However, after the unloading of the nanoindeneter, this system accounts for $60 \%$ of the total immobile dislocation-density evolution. At the final unloading point, the immobile dislocationdensities are highly localized on either side of the indenter. This clearly indicates that during unloading, different slip-systems are activated, and this is clearly related to the residual strains due to inelastic activity that have evolved within the aggregate during nanoindentation. This activation of different slip-systems occurs as the immobile dislocationdensity on the most active slip-system continues to increase up to unloading.

The accumulated plastic strains and pressure distribution at the final loading and unloading steps are shown in Figure 7.4. The highest plastic strains of 2.26 are in the regions corresponding to the highest immobile dislocation-density regions at the final loading step. The highest negative normalized pressures, with a value of -14.24 , occur also in the region of the highest immobile dislocation-densities on the most active slip-system.

\subsection{Effects of Increased Indentation Depths: Model 1}

We investigated the effects of increasing the indentation depth by increasing the normalized depth to $12.8 \%$ and $23.1 \%$ for Model 1 . At a normalized depth of $12.8 \%$, slipsystem 5 had the highest immobile dislocation-density of $3.88 \times 10^{16} \mathrm{~m}^{-2}$ (Figure $7.5 \mathrm{a}$ ) at the final loading step, which is approximately twice the value of the immobile dislocation- 
density for the $9 \%$ indentation case. The total immobile dislocation-density had evolved to $4.35 \times 10^{16} \mathrm{~m}^{-2}$ (Figure 7.5b). At unloading, the most active slip-system had an immobile dislocation-density of $7.46 \times 10^{16} \mathrm{~m}^{-2}$ (Figure $7.5 \mathrm{c}$ ), and the total immobile dislocation-density is $1.23 \times 10^{17} \mathrm{~m}^{-2}$ (Figures $7.5 \mathrm{~d}$ ). Hence, at the final loading point, the most active slip-system account for $89 \%$ of the immobile dislocation-density activity, and during unloading it accounts for $60.7 \%$ of immobile dislocation-density activity. This indicates that slip-systems continue to be activated, as the nanoindenter is unloaded. The maximum plastic strain at the maximum loading point is 2.62 for $12.8 \%$ (Figure $7.6 \mathrm{a}$ ). The plastic strains are accumulated in a continuous region, even though the immobile dislocation-densities are highly localized. The maximum pressures of (Figure 7.6c) are concentrated under the indenter and are in the same region where the maximum immobile dislocation-densities are. As also seen, negative pressures are also forming at the top of the specimen.

At a normalized indentation depth of $23.1 \%$, the immobile dislocation-density is $6.17 \times 10^{16} \mathrm{~m}^{-2}$, at the final loading step, for most active slip-system 12 (Figure 7.7a). The total immobile dislocation-density is $7.98 \times 10^{16} \mathrm{~m}^{-2}$ (Figure 7.7b). At the final unloading point, the immobile dislocation-density for slip-system 12 is $7.45 \times 10^{17} \mathrm{~m}^{-2}$ (Figure $7.7 \mathrm{c}$ ) and $1.02 \times 10^{18}$ $\mathrm{m}^{-2}$ for all slip-systems (Figure 7.7d). As with other indentation depths, the portion of active systems decreases from $77.3 \%$ at the final loading point to $73 \%$ during unloading step. Therefore, as the indented depth increases, and the number of indented grains increases, the residual strains in the deformed grains result in slip-system activation and inelastic activities. This is consistent with experimental observations (see, for example, Choi, Van Vliet et al., 2003), which clearly indicate that dislocations and strains accumulate within the specimen after the nanoindenter is unloaded. This is further substantiated by the accumulation of 
plastic strains (Figure 7.8a), which attain a maximum of 2.84 (2.26 for 9\% and 2.62 for $12.8 \%)$ at the final loading step and the pressures, which attain the highest negative normalized values of -24 at the maximum loading point (Figure 7.8c).

\subsection{Effects of Increased Aggregate Sizes: Model 2}

In this aggregate, we decreased the grain-size to compare the effects with the largersized grains of Model 1. At a normalized indentation depth of 9\%, slip-system 3 was the most active at the final loading step, and it had $2.61 \times 10^{15} \mathrm{~m}^{-2}$ as an immobile dislocationdensity, (Figure 7.9a), the total immobile dislocation-density is $5.68 \times 10^{15} \mathrm{~m}^{-2}$ (Figure 7.9b). Hence, the most active slip-system accounted for $56 \%$ of the immobile dislocation-density activity. The immobile dislocation-density, at the final unloading, is $1.71 \times 10^{16} \mathrm{~m}^{-2}$ (Figure $7.9 \mathrm{c})$ and $4.51 \times 10^{16} \mathrm{~m}^{-2}$ for the total immobile dislocation-density (Figures $7.9 \mathrm{~d}$ ). The immobile dislocation-density of the most active slip-system accounts for $38 \%$ of the total immobile dislocation-density evolution during unloading. This is a much smaller portion of the immobile dislocation-density evolution in comparison with the larger grain-size models (Model 1). This is directly related to that more grains are used in this aggregate, which means that collectively more slip-systems are potentially activated due to that more grains are indented. The accumulated plastic strains and pressure distributions at the maximum loading and unloading points are shown in Figure 7.10. The pressures have the highest negative normalized value of -16.81 , at the final loading point, and the highest positive normalized value of 21.97 at the final unloading point. These are also the regions where the maximum immobile dislocation-densities were accumulated during loading and unloading. 
Furthermore, it is also in the region where the maximum inelastic strains of 1.54 at the final loading point and 2.48 at the unloading point have accumulated.

\subsection{Crystalline Orientation Effects}

To further understand the spatial and angular variation of critical quantities, such as the immobile dislocation-densities and the plastic strains, we evaluated different quantities at different angular orientations and distances with respect to the indented surface. Three angles of $30^{\circ}, 45^{\circ}$, and $60^{\circ}$ were defined with respect to the indentation-loading axis (Figure 7.11). A distance, which was normalized by the vertical length, from the indentation contact point to the any point inside the grain or GB, was also defined. Hence, we could investigate grain and GB behavior at critical locations and orientations. The immobile dislocation-density variation for Model 1 is shown in Figure 7.12 at different normalized depths. For all three normalized depths, the immobile dislocation-densities are highest directly under the indenter. There is also an angular symmetry for all three indented depths. At the largest indented depth, increased immobile dislocation-density activities can be seen at the GBs (Figure 7.12), which is due to the activation of different slip-systems within the GB region. As discussed earlier, this increase is due to the high-localized pressures and plastic strains near the GBs. The plastic strains are shown in Figure 7.13 and the pressures are shown in Figures 7.14. In these figures, the pressures and the plastic strains are symmetrically distributed with respect to the maximum indentation points. Furthermore, it is clearly seen that near the GBs, there are increases in the local accumulated plastic strains and pressures at locations that are between orientations of $30^{\circ}$ and $45^{\circ}$. These orientations are consistent with the maximum resolved 
shear stress orientations of $45^{\circ}$. Hence, the GB and crystalline orientations are the main mechanisms that can trigger dislocation-density activities due to nanoindentation, which is consistent with experimental observations by Ohmura, Minor et al. (2004).

\subsection{GB Transmission Factors}

In chapter 4, we presented the GB kinematic interfacial scheme, which can now be used to understand and predict different immobile dislocation-densities transmission or impedance through different GBs. The calculated transmission factors (TR) contour plots for GBs, near an indented surface, at a normalized indentation depth of $9 \%$, for Model 1, are shown in Figures 7.15. The highest immobile dislocation-density transmissions are at the left hand side, which is also the region with the highest pressures. This further substantiates that the hydrostatic pressures are a main factor for dislocation-density activity which is confirmed with the experimental observations of Hasnaoui, Derlet et al. (2004) related to the critical role that local pressures play in dislocation emission and absorption near GB regions.

\subsection{Summary and Conclusions}

This study provides a detailed understanding of the interrelated physical mechanisms that result in material behavior under nanoindentation in polycrystalline aggregates with low angle GB misorientations. A multiple-slip rate-dependent crystalline constitutive formulation that is coupled to the evolution of mobile and immobile dislocation-densities and specialized hierarchical computational schemes has been developed to investigate microstructually- 
induced behavior due to the nanoindentation of f.c.c. gold aggregates. The hierarchical scheme was used to scale the MD displacement profiles to the microstructural level. Low angle GB effects were accounted for by using random low angle misorientations for different sized aggregates and grains.

These low angle GB misorienations resulted in significantly different behavior than the cube-oriented aggregates. The predictions were used to determine how at local unloading, there was slip-system activation, at different indentation depths. Furthermore, it was shown how the immobile dislocation-densities evolve at the maximum loading and unloading points, and how the nominal residual strains are intricately related to the accumulation of plastic strains and pressures. In Table 7.3, we summarize for Model 1, at the maximum loading point, how the accumulated plastic strains, immobile dislocation-densities, and highest negative pressures are interrelated, and how these large values continue to accumulate during the loading of the nanoindenter.

Table 7.3. Immobile dislocation-density, plastic strains and pressure variation at final loading

\begin{tabular}{ccccc}
\hline $\begin{array}{c}\text { GB } \\
\text { Orientation }\end{array}$ & $\rho_{\text {im }}\left(\mathrm{m}^{-2}\right)$ & $\begin{array}{c}\text { Most Active } \\
\text { Slip-System }\end{array}$ & $\begin{array}{c}\text { Maximum } \\
\text { Plastic } \\
\text { Strains }\end{array}$ & $\begin{array}{c}\text { Maximum } \\
\text { Pressure }\end{array}$ \\
\hline $15^{\circ}(9 \%)$ & $2.08 \times 10^{16}$ & 5 & 2.26 & -14.24 \\
$15^{\circ}(12.8 \%)$ & $3.88 \times 10^{16}$ & 5 & 2.62 & -15.25 \\
$15^{\circ}(23.1 \%)$ & $6.17 \times 10^{16}$ & 12 & 2.84 & -24 \\
\hline
\end{tabular}

We were able to identify the exact locations and orientations of the immobile dislocation-densities, plastic strains, and pressures from the indentation surface. These values generally decreased, as the normalized distance from the indenter contact point increased. At the maximum indentation point, there was a sharp increase of immobile dislocation-density 
and plastic strains near specific GBs and surface areas. This can be due by the bending effects associated with larger indentation depth (see, for example, Zimmerman, Kelchner et al. 2001, Knap and Ortiz 2003, and Greer and Nix 2005).

Knowing that the GBs at specific locations played a dominant role in immobile dislocation-density evolution and slip-system switching, we used the GB interfacial scheme to determine exactly where and how immobile dislocation-densities transmitted or where impeded. Our predictions indicate that the high GB transmission rates are directly related to the high pressures near the GB regions. Hence, we could relate how local pressures at specific GB locations and orientations affect immobile dislocation-density evolution during nanoindentation.

This study underscores how the hierarchical scheme, the dislocation-density based formulation, and the GB interfacial kinematics can provide a comprehensive understanding of the effects of multiple-slip inelastic deformation and dislocation interactions, which are associated with nanoindenation in polycrystalline aggregates with low angle GB misorientations. Critical GB and grain locations can be identified, and hence these predictions can be used to obtain desired microstructural behavior. 


\section{References}

Ashmawi, W. M. and M. A. Zikry (2000). "Effects of grain boundaries and dislocation density evolution on large strain deformation modes in fcc crystalline materials." Journal of Computer-Aided Materials Design 7(1): 55-62.

Choi, Y., K. J. Van Vliet, et al. (2003). "Size effects on the onset of plastic deformation during nanoindentation of thin films and patterned lines." Journal of Applied Physics 94(9): 6050-6058.

Greer, J. R. and W. D. Nix (2005). "Size dependence of mechanical properties of gold at the sub-micron scale." Applied Physics a-Materials Science \& Processing 80(8): 16251629.

Hasnaoui, A., P. M. Derlet, et al. (2004). "Interaction between dislocations and grain boundaries under an indenter - a molecular dynamics simulation." Acta Materialia 52(8): 2251-2258.

Knap, J. and M. Ortiz (2003). "Effect of indenter-radius size on Au(001) nanoindentation." Physical Review Letters 90(22).

Michalske, T. A. and J. E. Houston (1998). "Dislocation nucleation at nano-scale mechanical contacts." Acta Materialia 46(2): 391-396.

Ohmura, T., A. M. Minor, et al. (2004). "Dislocation-grain boundary interactions in martensitic steel observed through in situ nanoindentation in a transmission electron microscope." Journal of Materials Research 19(12): 3626-3632.

Zimmerman, J. A., C. L. Kelchner, et al. (2001). "Surface step effects on nanoindentation." Physical Review Letters 8716(16): art. no.-165507. 


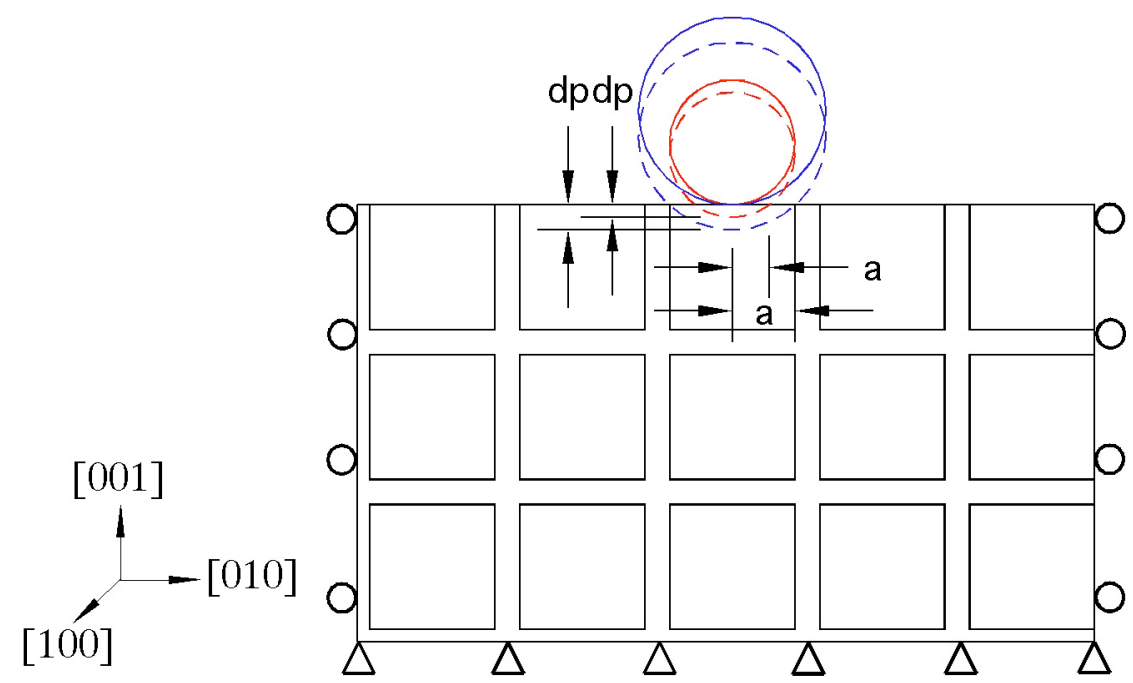

$\mathrm{dp}$ : normalized indentation depth

a : contact area

\begin{tabular}{cccc}
$\varphi_{1}$ & $\Phi$ & $\varphi_{1}$ & $\left(^{\circ}\right)$ \\
\hline 0 & 0 & 0 & \\
3.33 & 4.49 & 5.86 & \\
9.10 & 9.79 & 0.90 \\
2.24 & 6.66 & 0.04 \\
14.64 & 2.81 & 7.73 \\
$\cdot$ & $\cdot$ & $\cdot$ \\
$\cdot$ & $\cdot$ & $\cdot$ \\
$\cdot$ & $\cdot$ & $\cdot$ \\
. & $\cdot$ &.
\end{tabular}

Figure 7.1. Polycrystalline aggregate, stress axis, and random low angle GB misorientations used in FEA 


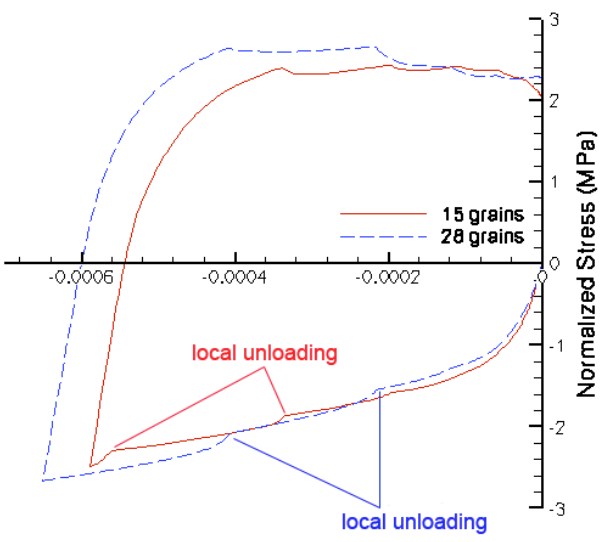

Nomınal Straın

(a)

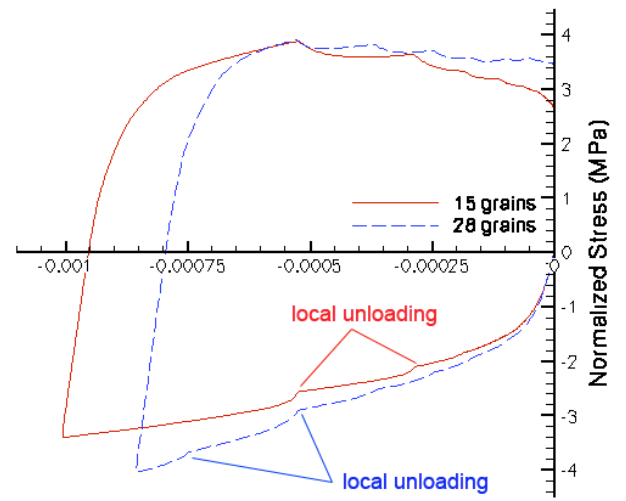

Nomınal Straın

(b)

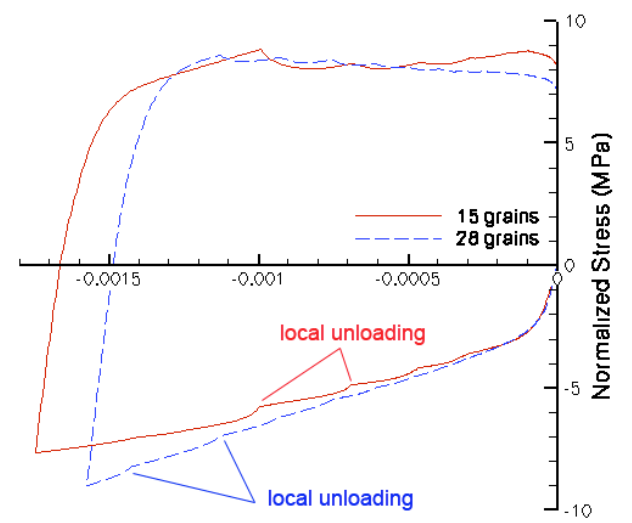

Nomınal Straın

(c)

Figure 7.2. Stress-strain curves for gold polycrystalline aggregates with different normalized indented depths, aggregate sizes, and low angle misorientations (a) $9 \%$ (b) $12.8 \%$ (c) $23.1 \%$ 


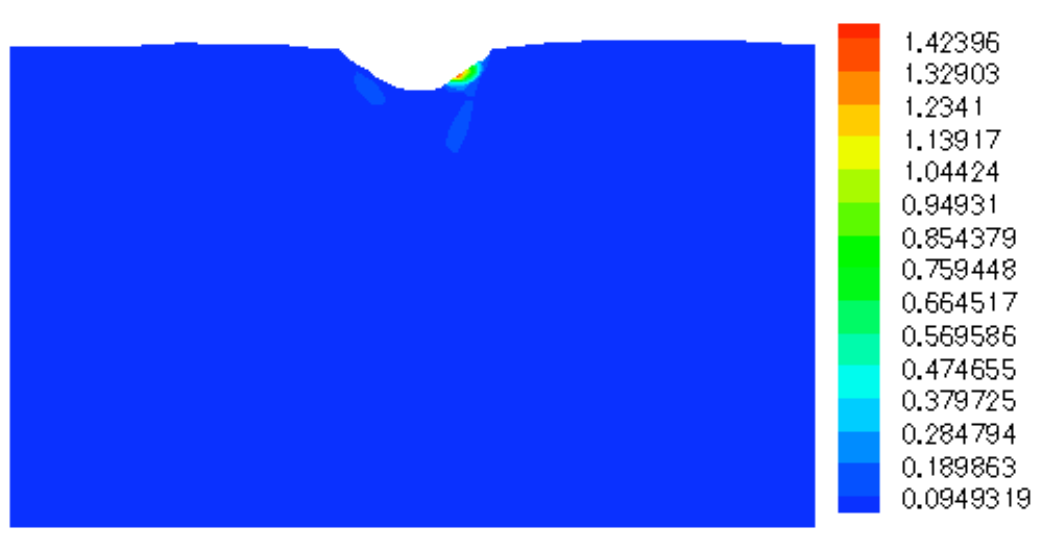

(a)

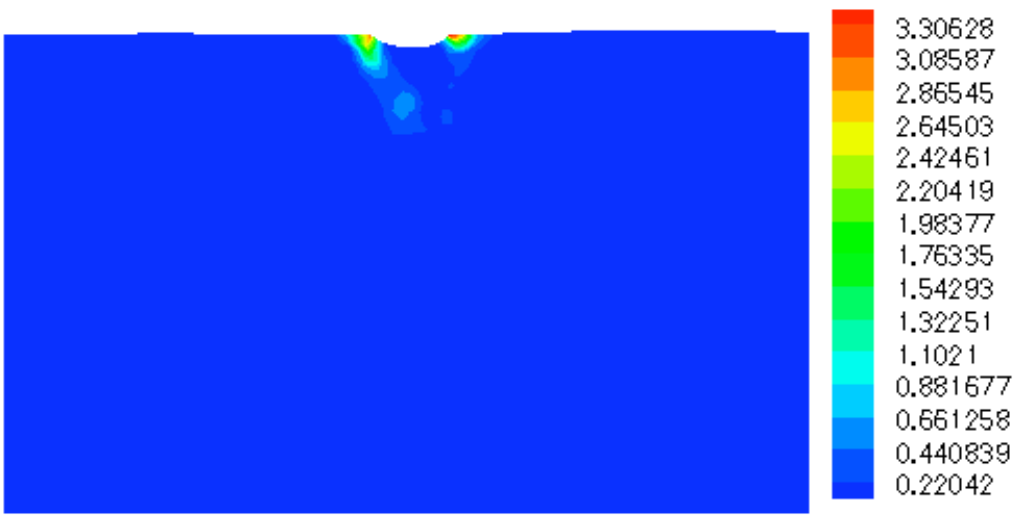

(c)

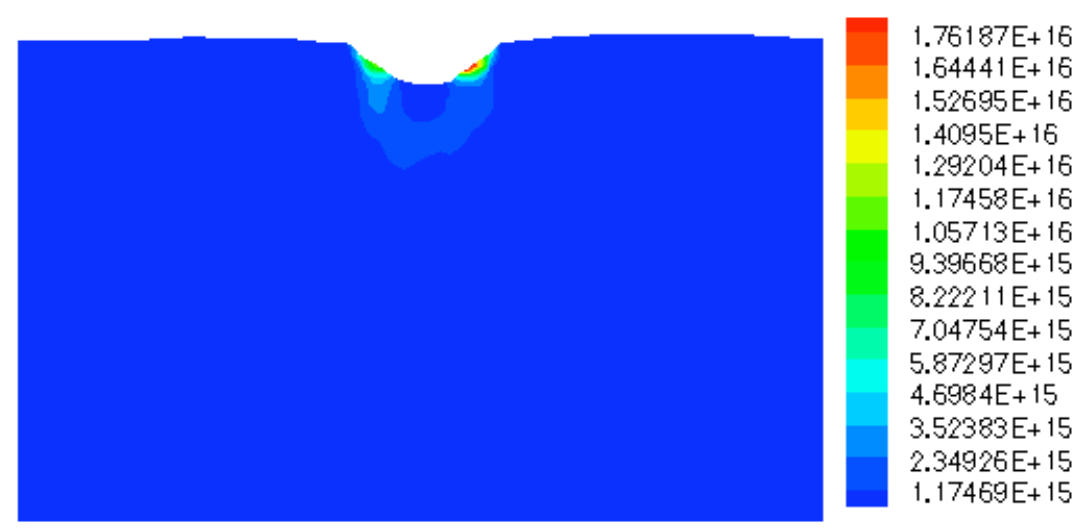

(b)

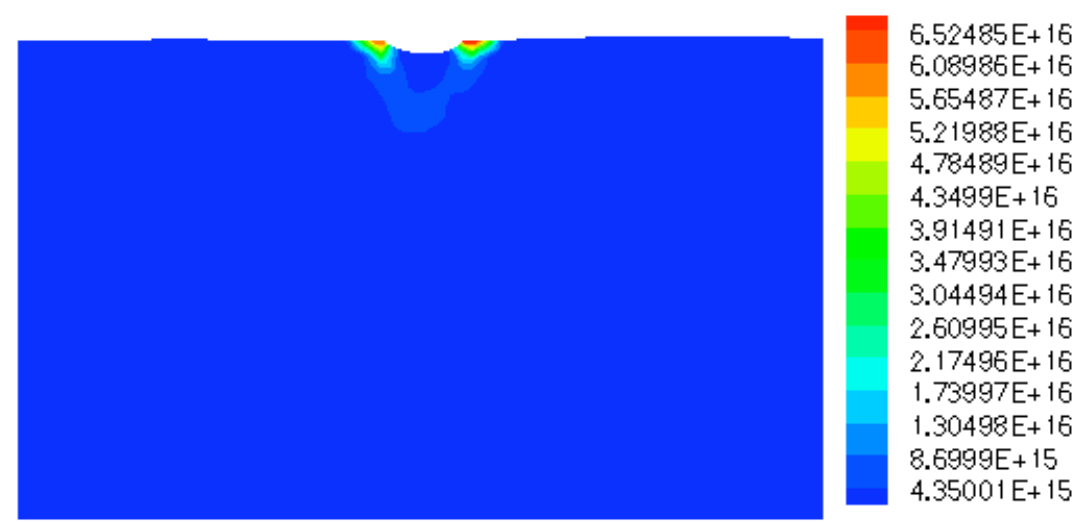

(d)

Figure 7.3. Immobile dislocation-density at final loading step for system 5 normalized by saturation (a), total immobile dislocationdensity at final loading step non-normalized (b), just before unloading step for system 5 normalized by saturation (c), and total immobile dislocation-density just before unloading step non-normalized (d). (Model 1 with 9\% indentation) 


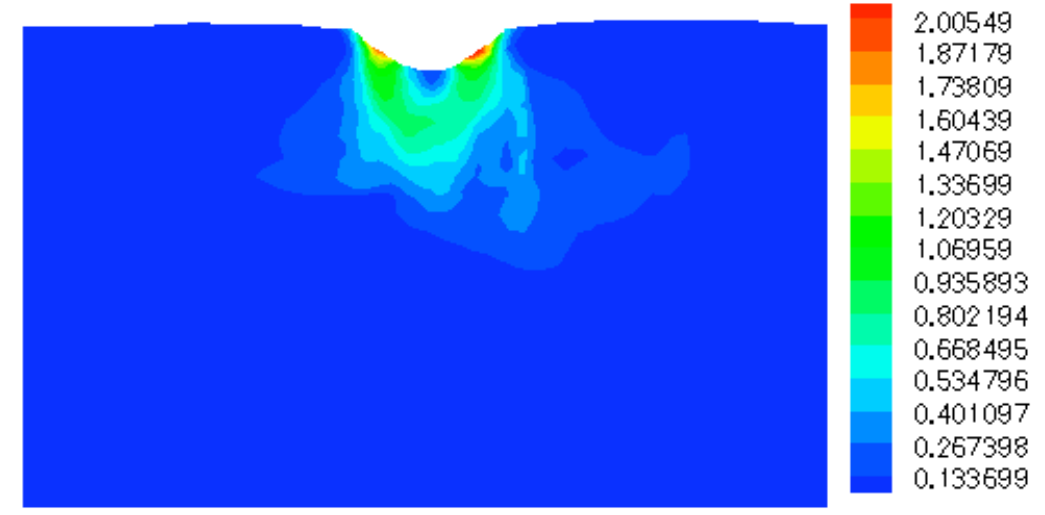

(a)

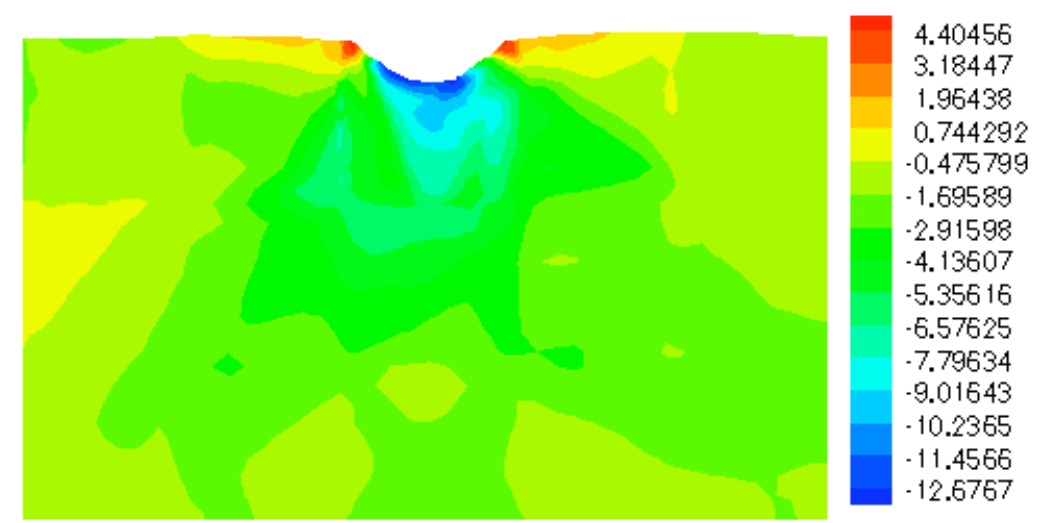

(c)

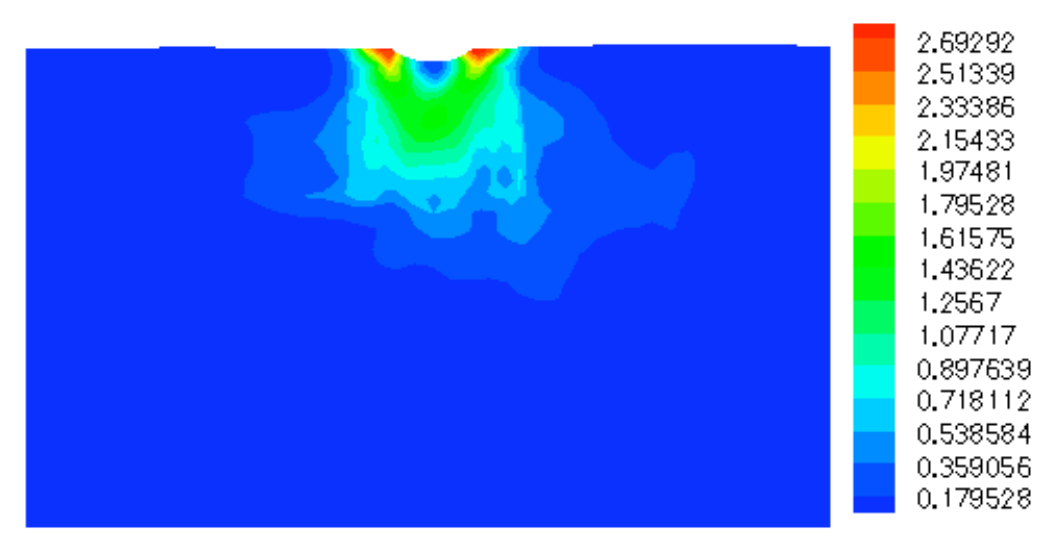

(b)

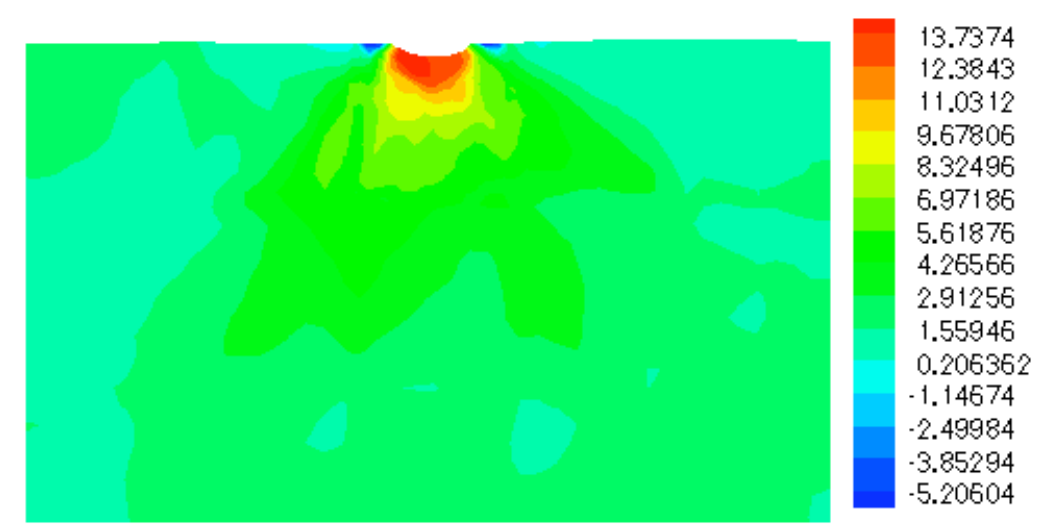

(d)

Figure 7.4. Plastic strain accumulation at final loading step (a), just before unloading step (b), pressure at final loading step normalized by yield stress (c), and pressure just before unloading step normalized by yield stress (d). (Model 1 with $9 \%$ indentation) 


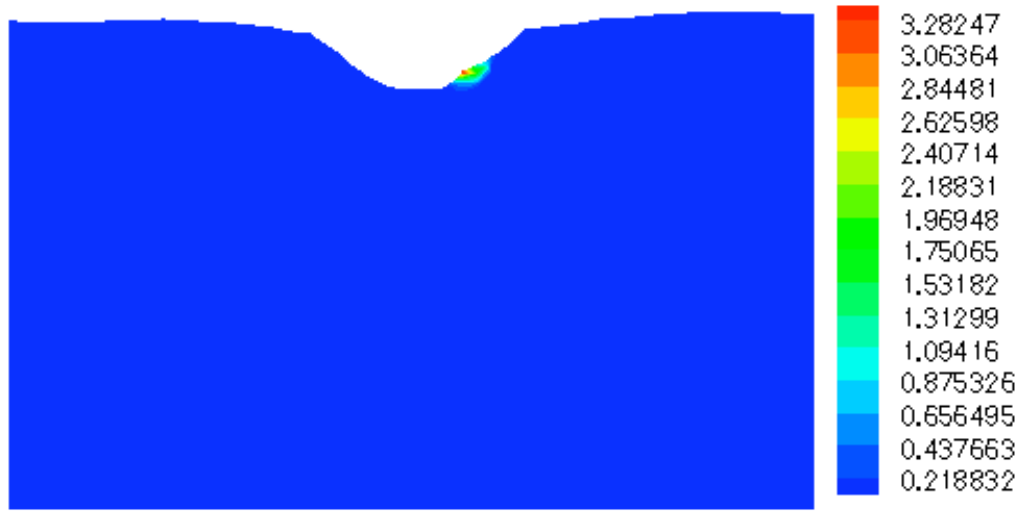

(a)

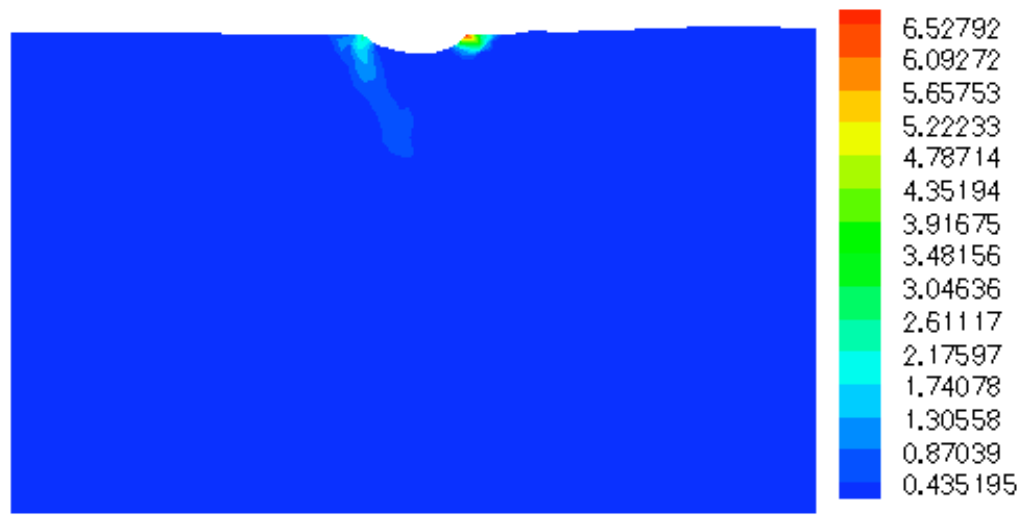

(c)

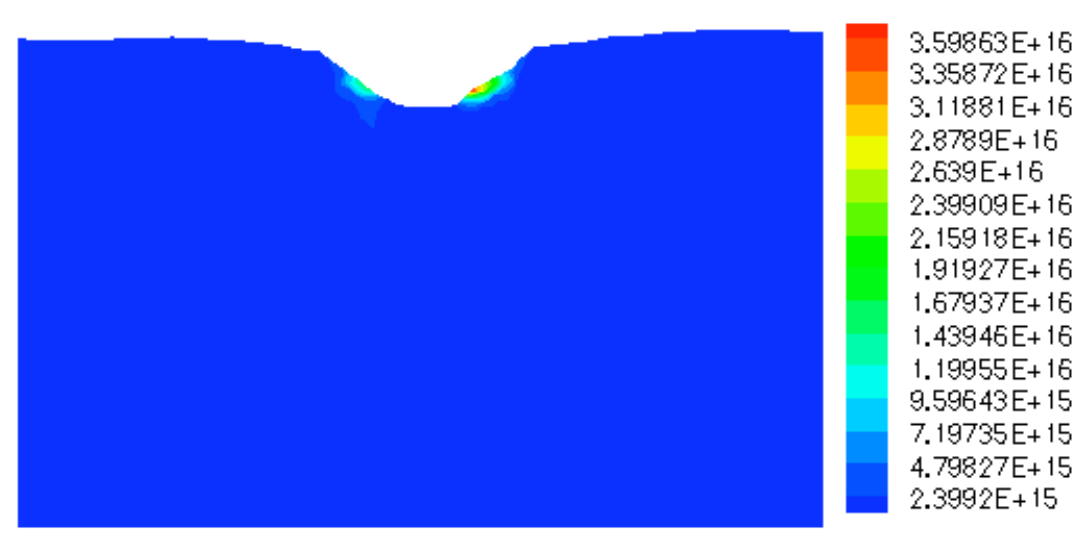

(b)

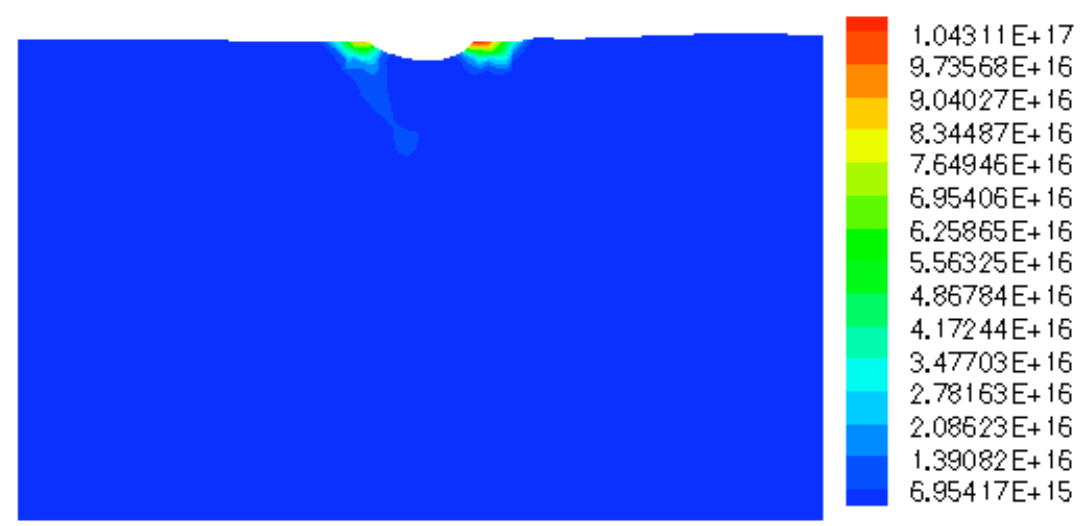

(d)

Figure 7.5. Immobile dislocation-density at final loading step for system 5 normalized by saturation (a), total immobile dislocationdensity at final loading step non-normalized (b), just before unloading step for system 5 normalized by saturation (c), and total immobile dislocation-density just before unloading step non-normalized (d). (Model 1 with $12.8 \%$ indentation) 


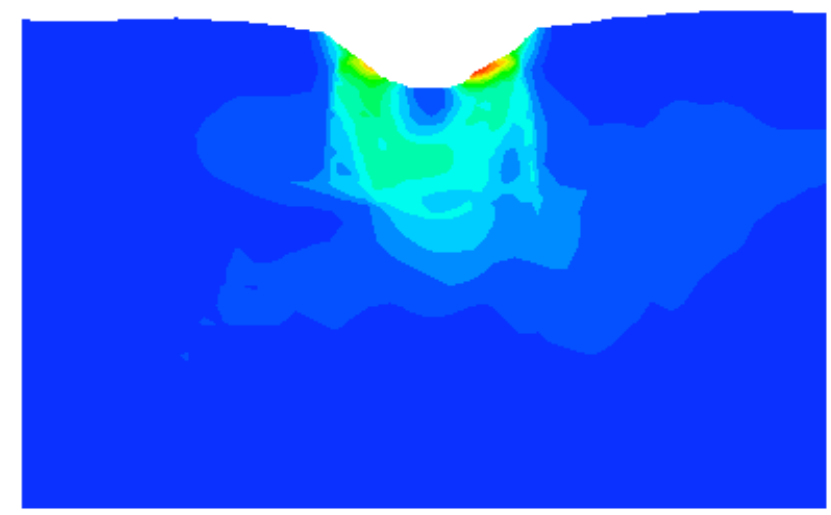

(a)

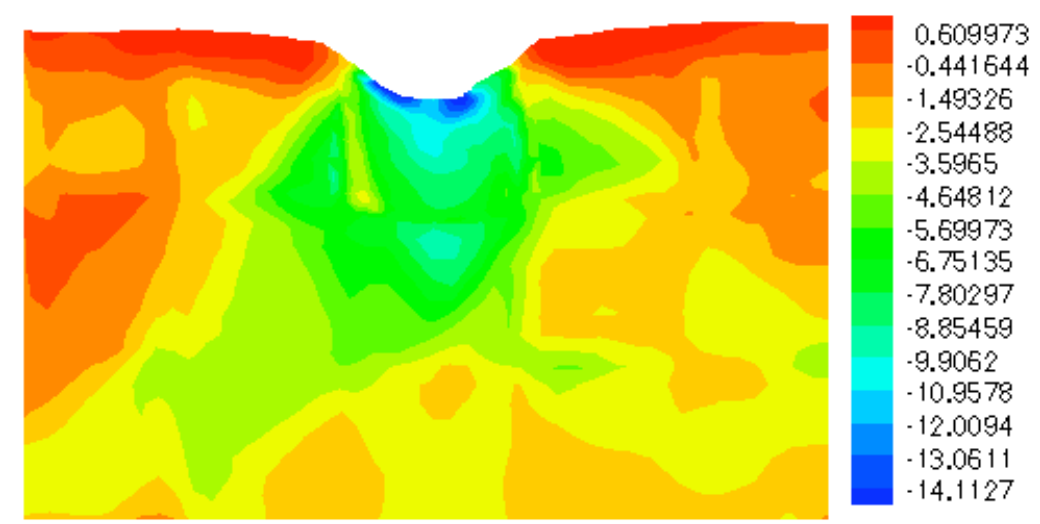

(c)

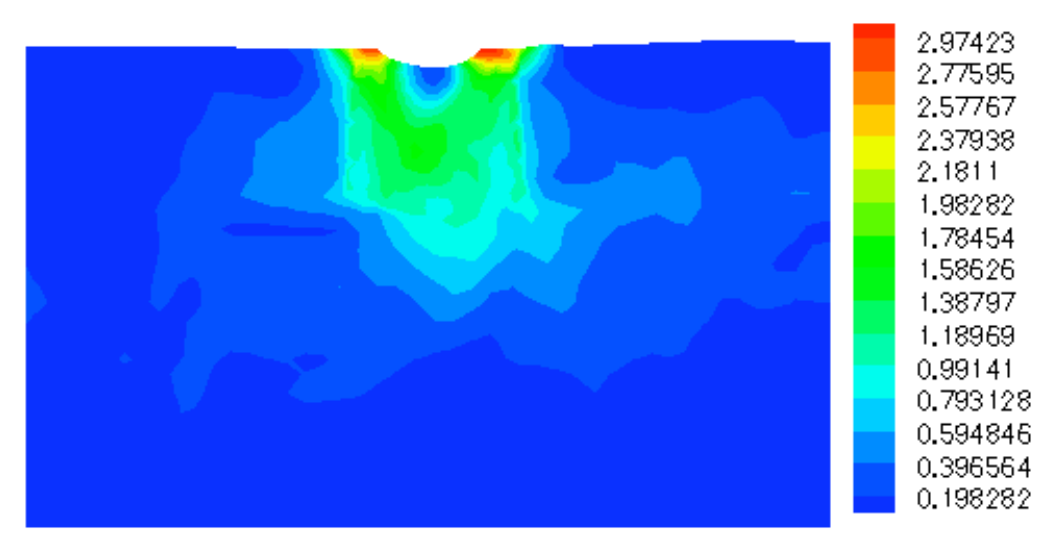

(b)

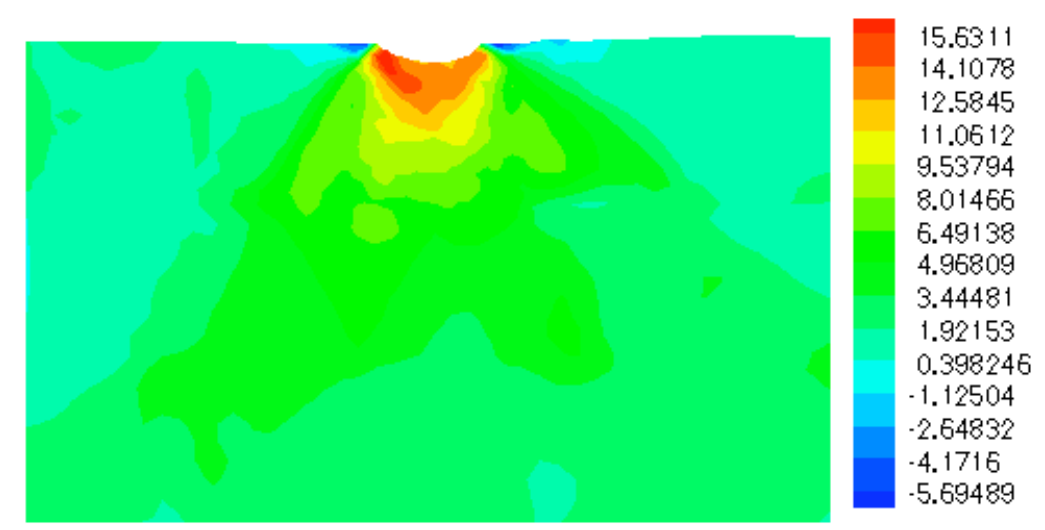

(d)

Figure 7.6. Plastic strain accumulation at final loading step (a), just before unloading step (b), pressure at final loading step normalized by yield stress (c), and pressure just before unloading step normalized by yield stress (d). (Model 1 with 12.8\% indentation) 


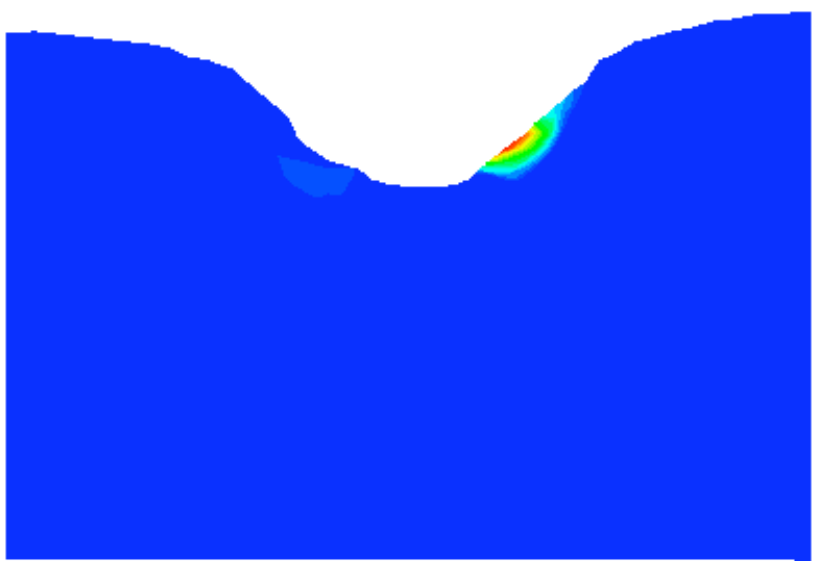

(a)

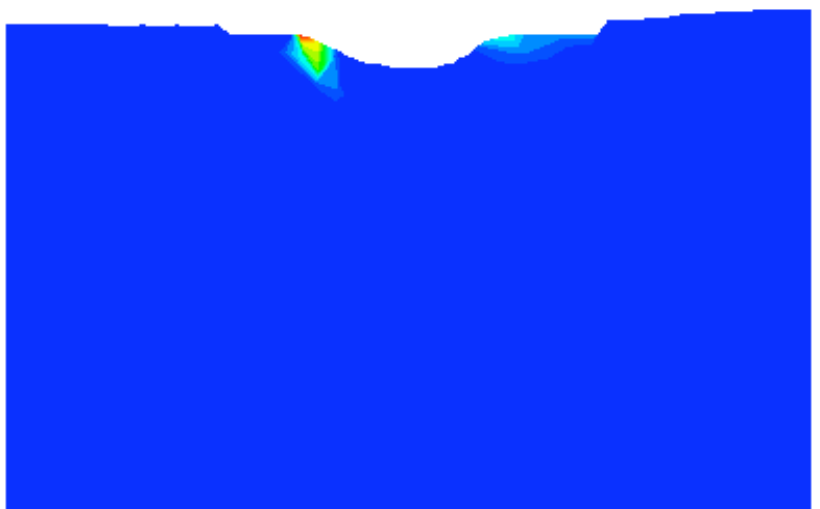

(c)

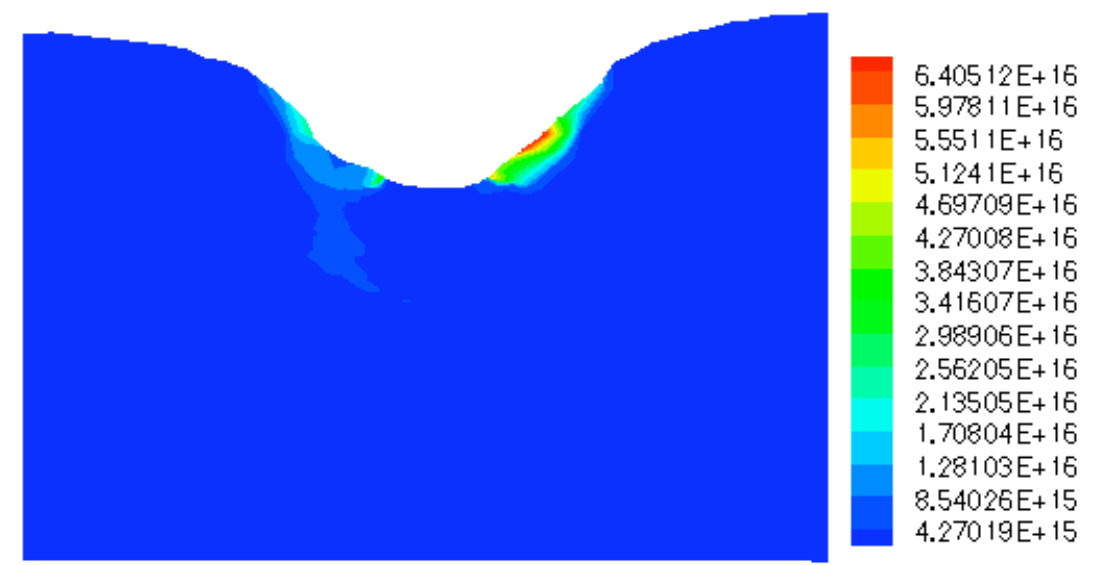

(b)

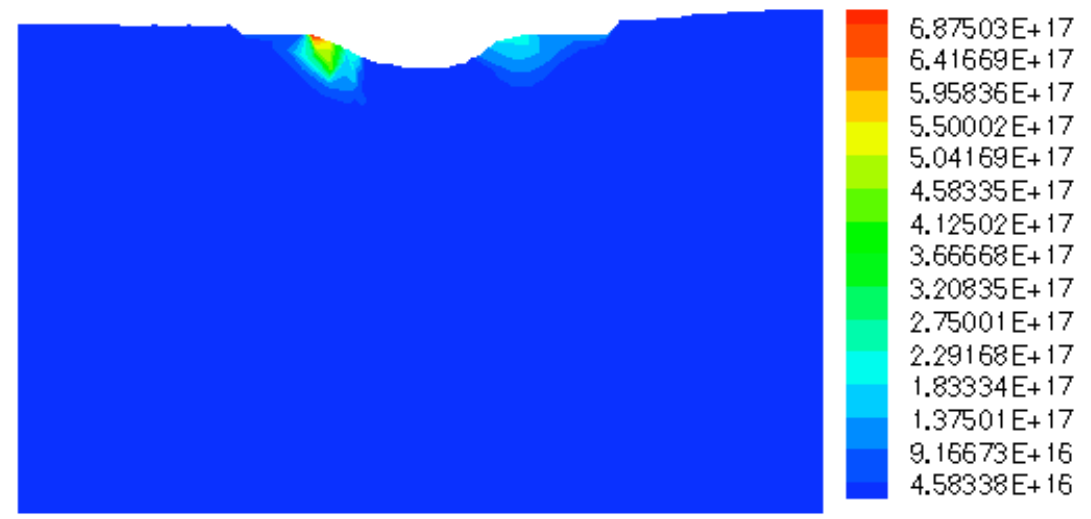

(d)

Figure 7.7. Immobile dislocation-density at final loading step for system 12 normalized by saturation (a), total immobile dislocationdensity at final loading step non-normalized (b), just before unloading step for system 12 normalized by saturation (c), and total immobile dislocation-density just before unloading step non-normalized (d). (Model 1 with $23.1 \%$ indentation) 


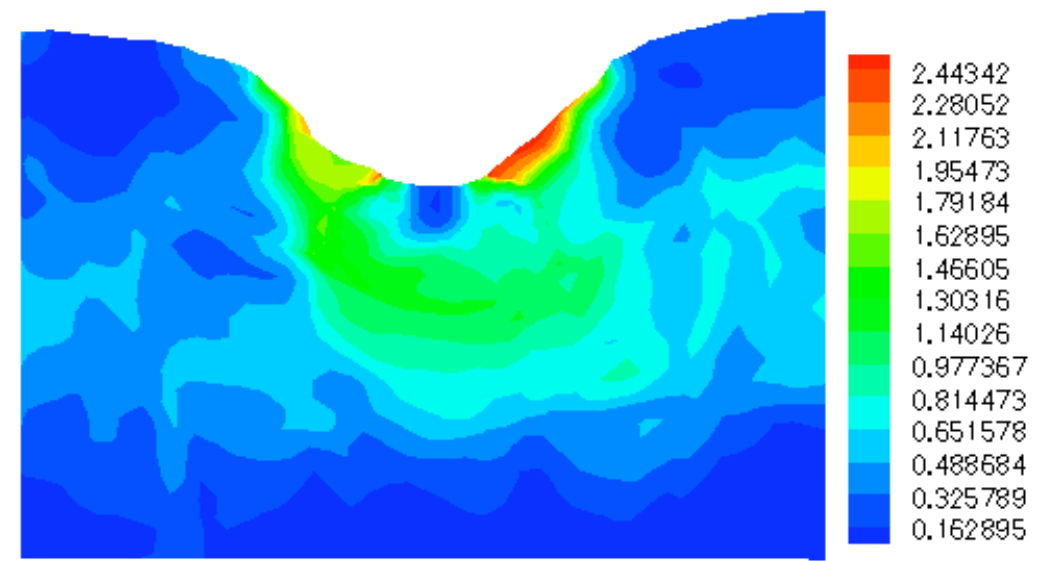

(a)

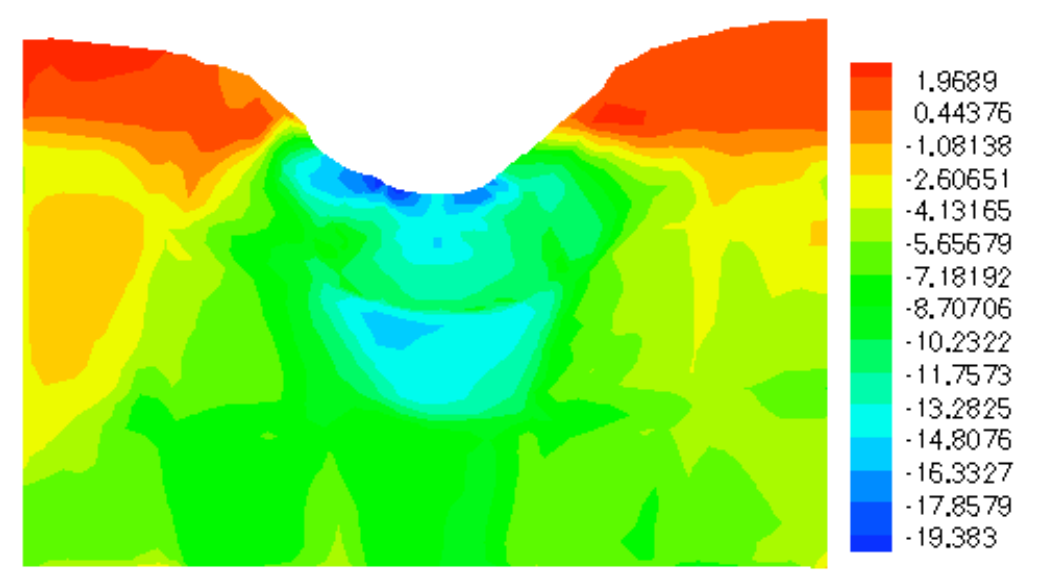

(c)

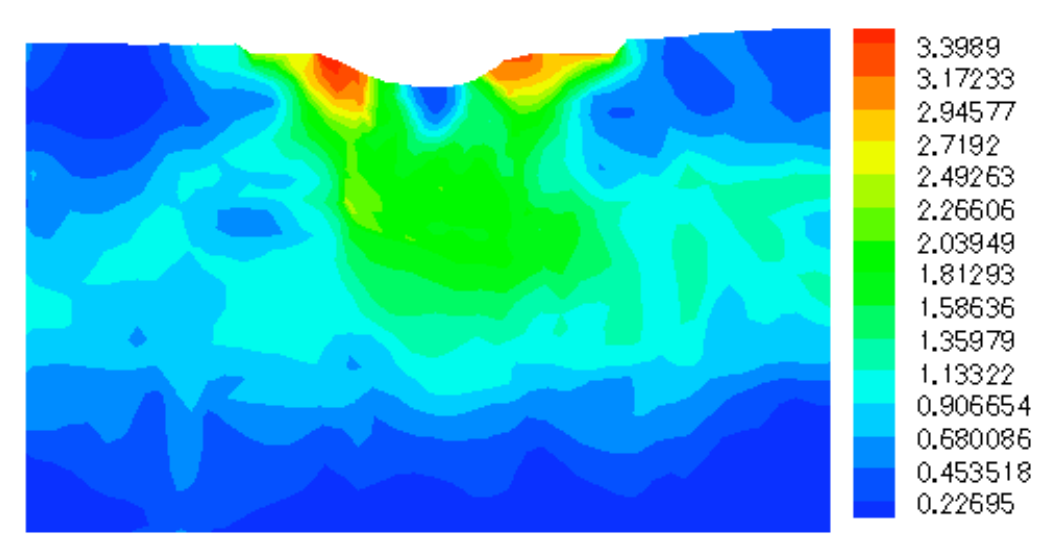

(b)

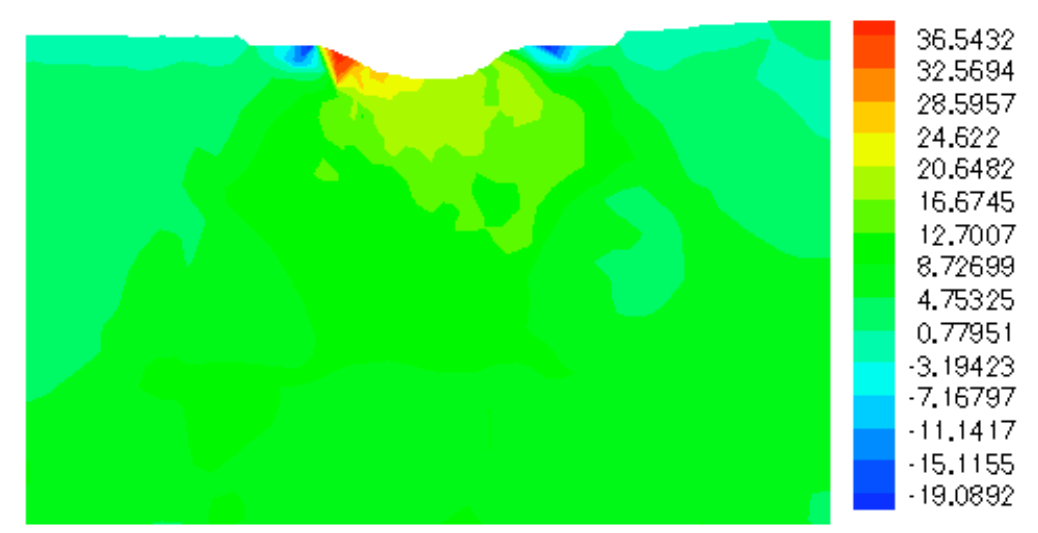

(d)

Figure 7.8. Plastic strain accumulation at final loading step (a), just before unloading step (b), pressure at final loading step normalized by yield stress (c), and pressure just before unloading step normalized by yield stress (d). (Model 1 with $23.1 \%$ indentation) 


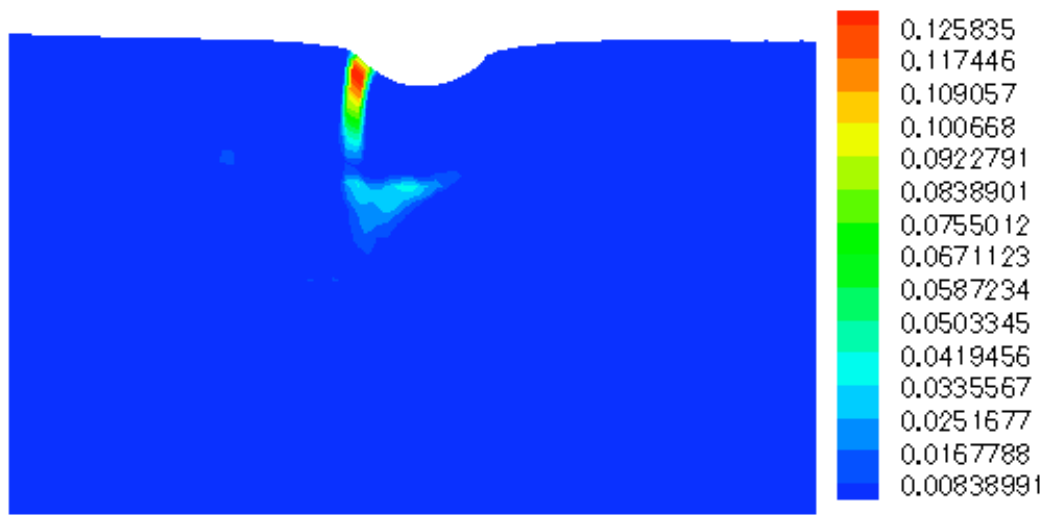

(a)

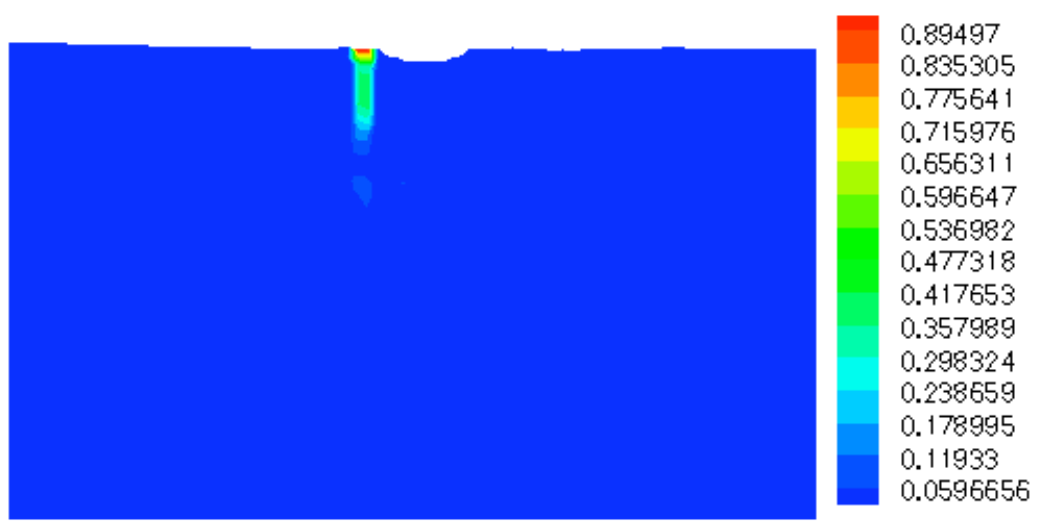

(c)

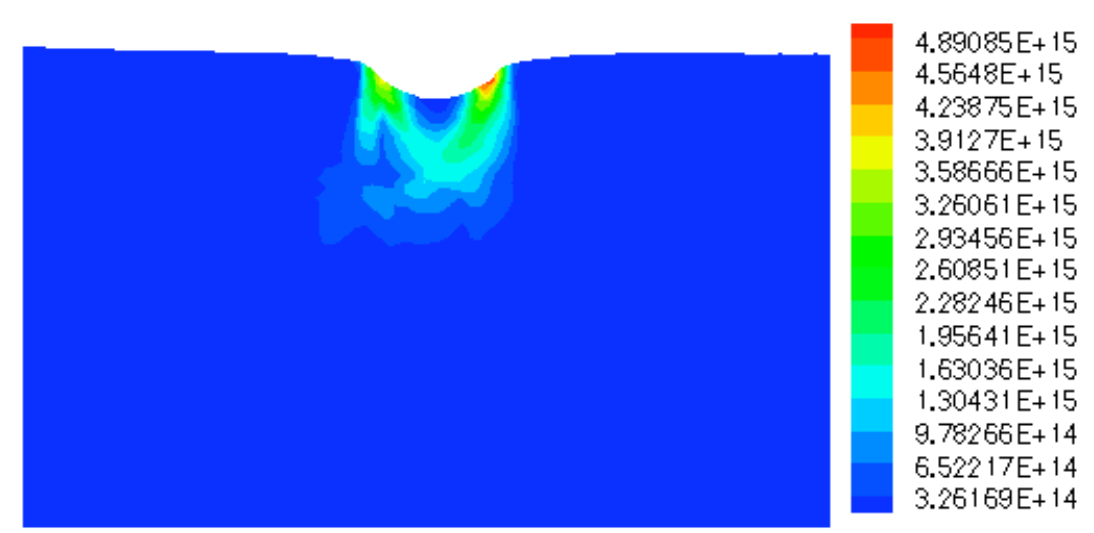

(b)

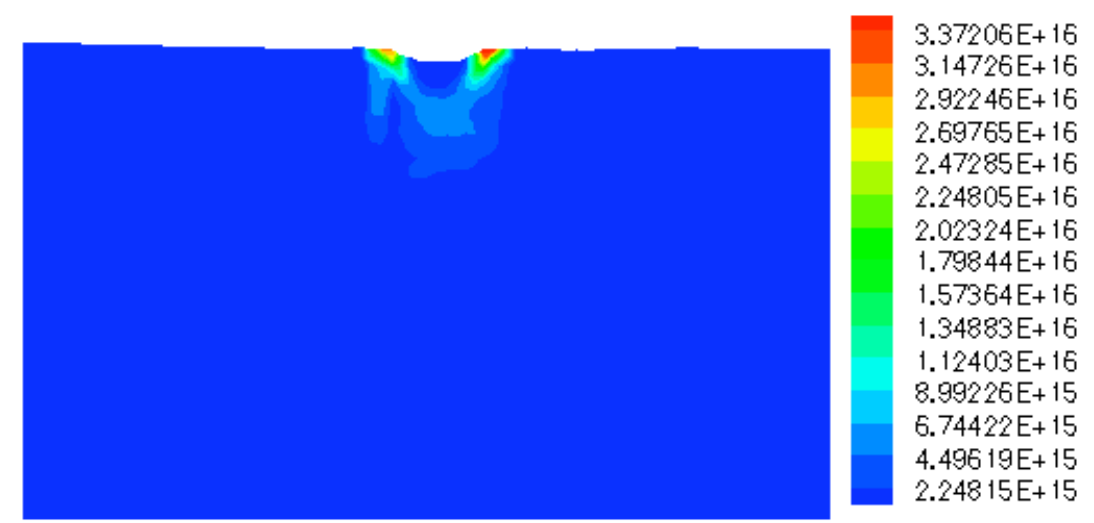

(d)

Figure 7.9. Immobile dislocation-density at final loading step for system 3 normalized by saturation (a), total immobile dislocationdensity at final loading step non-normalized (b), just before unloading step for system 3 normalized by saturation (c), and total immobile dislocation-density just before unloading step non-normalized (d). (Model 2 with 9\% indentation) 


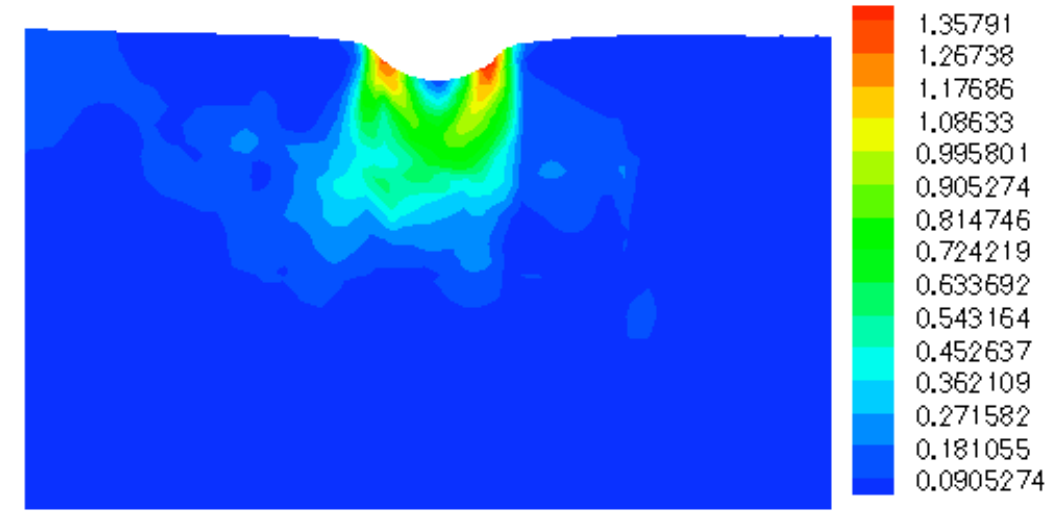

(a)

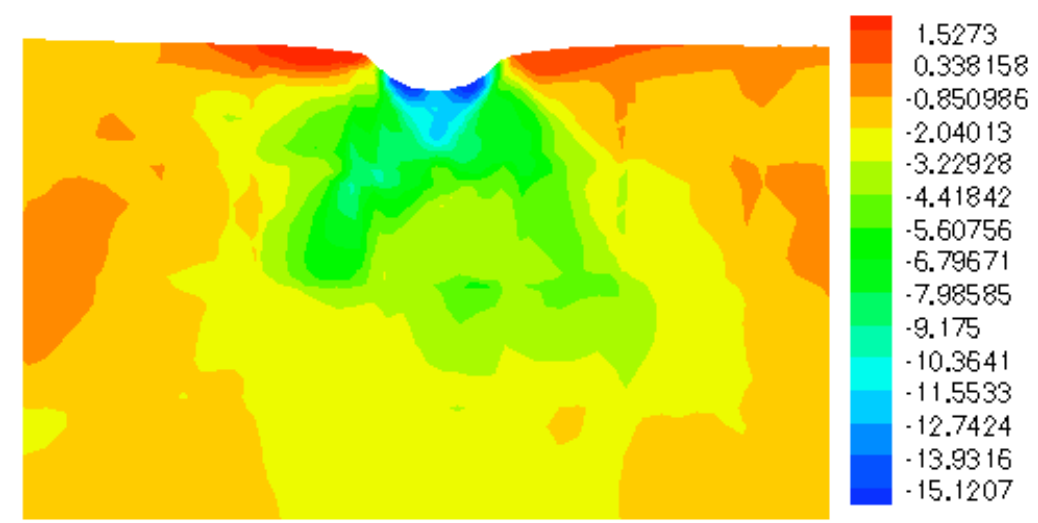

(c)

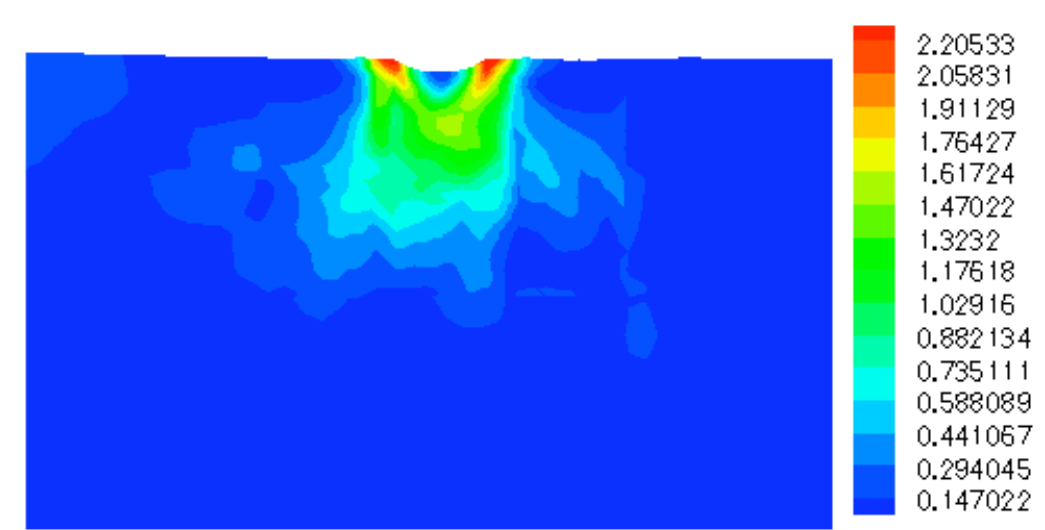

(b)

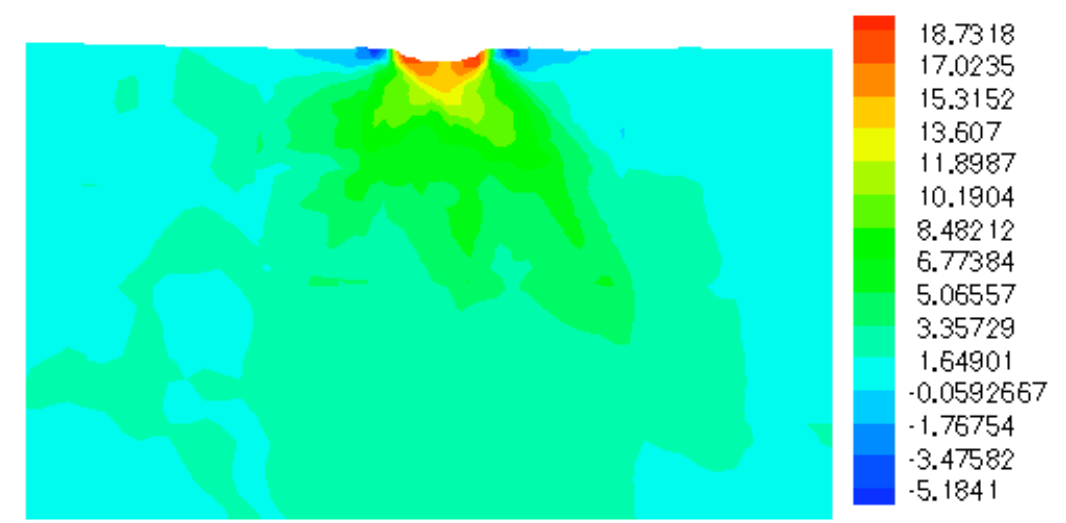

(d)

Figure 7.10. Plastic strain accumulation at final loading step (a), just before unloading step (b), pressure at final loading step normalized by yield stress (c), and pressure just before unloading step normalized by yield stress (d). (Model 2 with $9 \%$ indentation) 


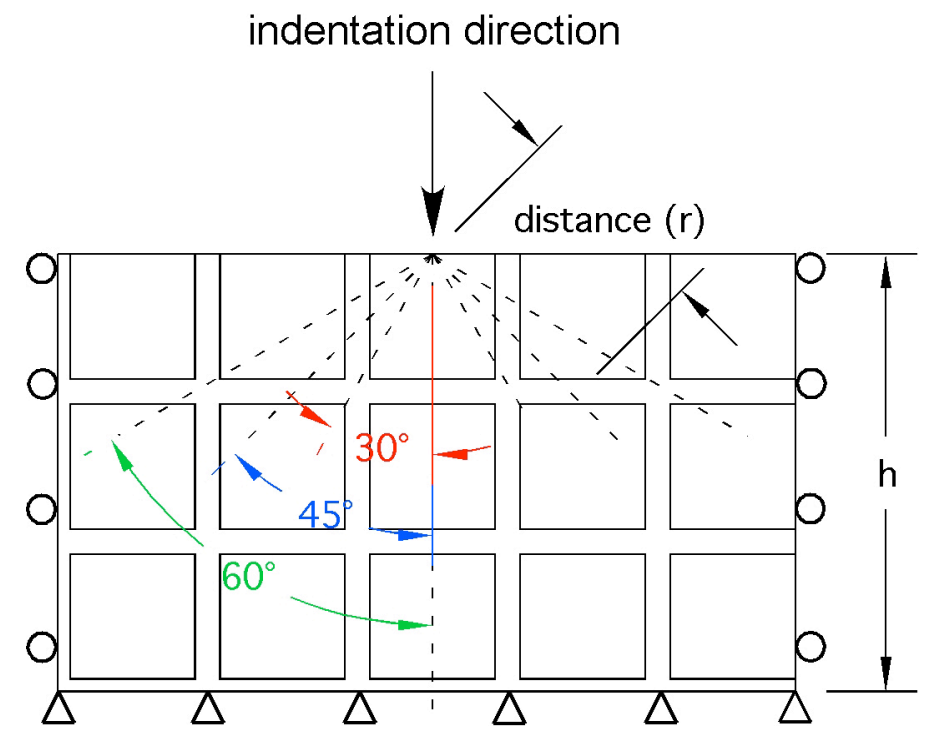

Figure 7.11. Three angular directions with respect to the indentation-loading axis 


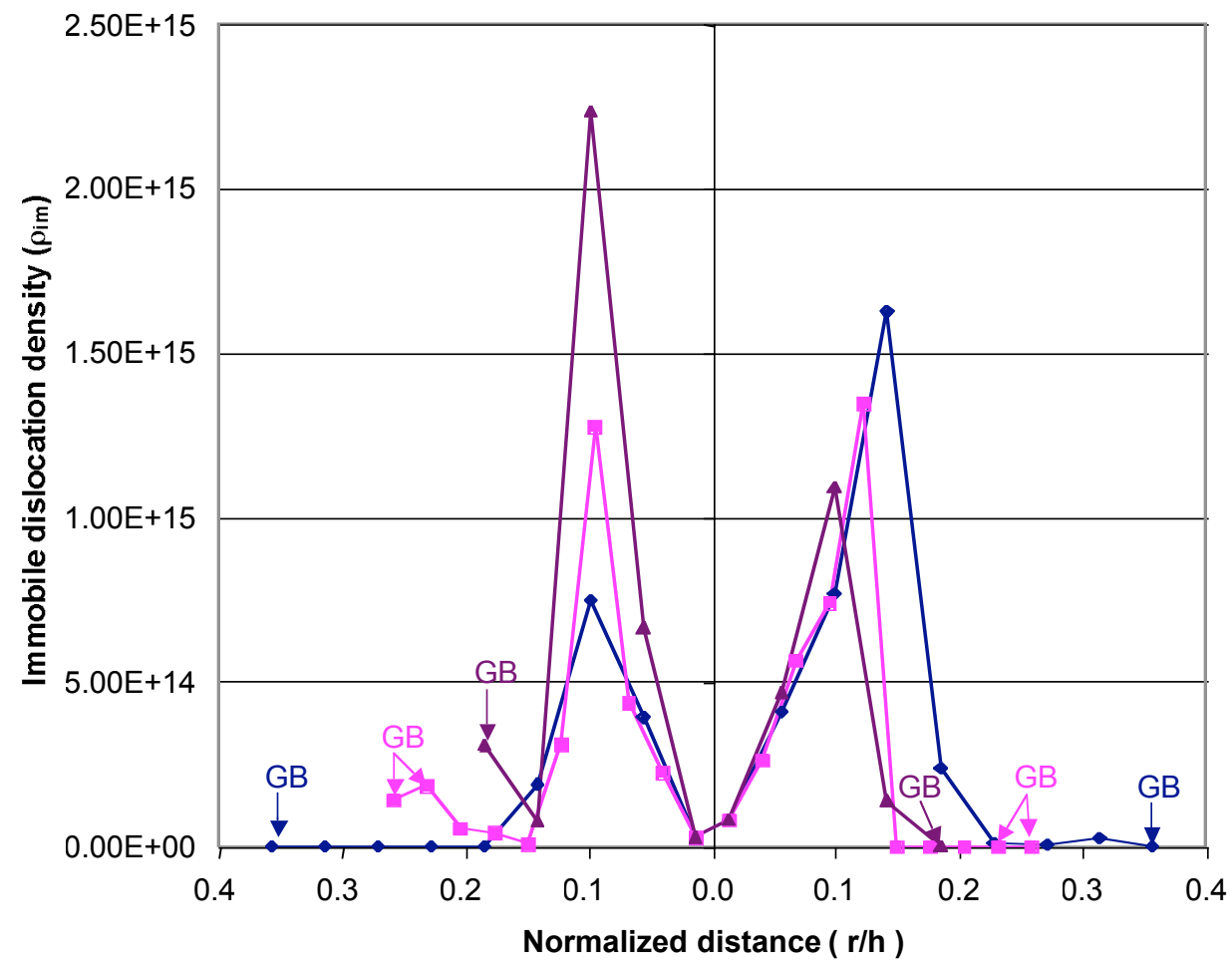

$\multimap$ (a)30deg

- - (b)45deg

- (c)60deg

(i)

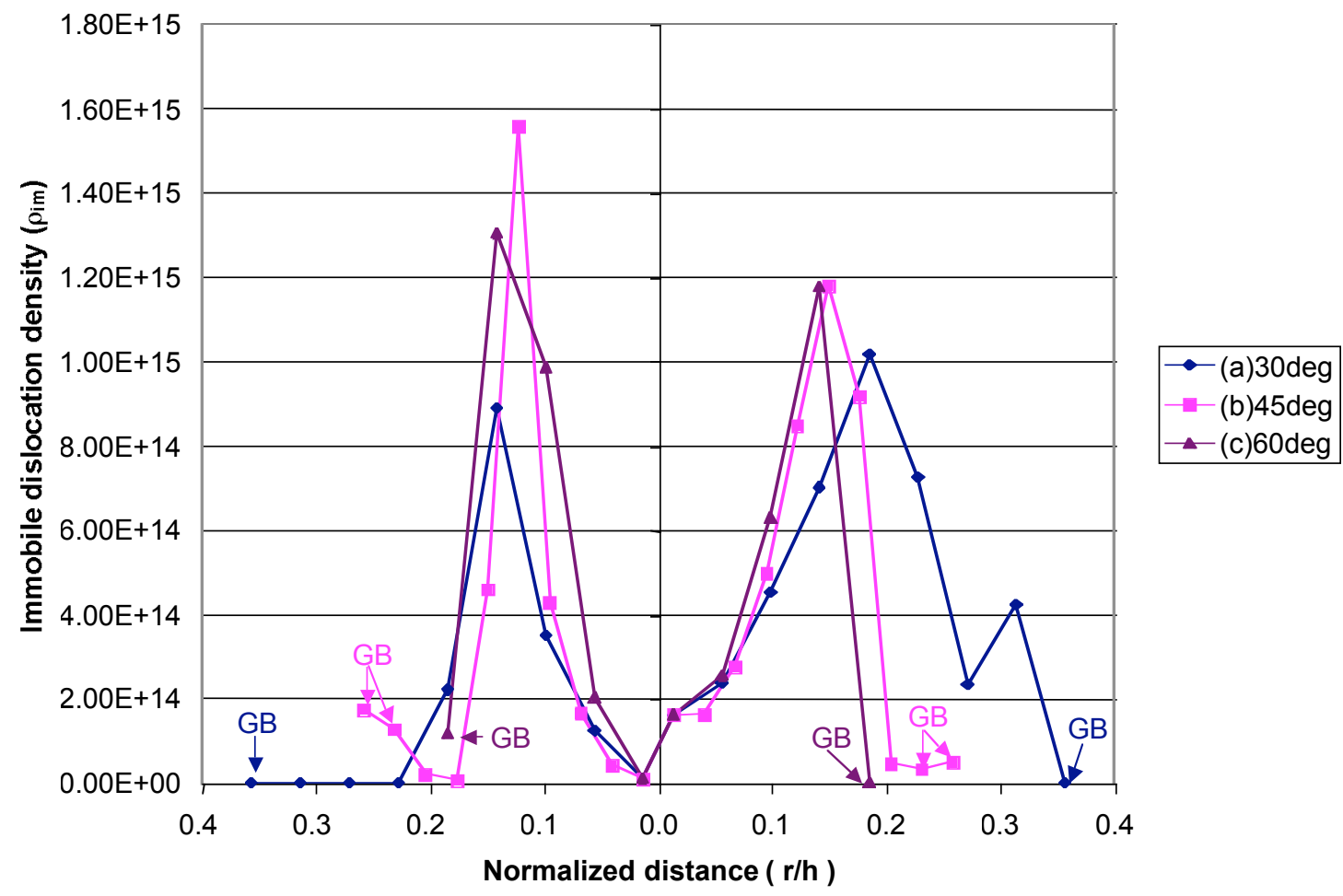

(ii) 


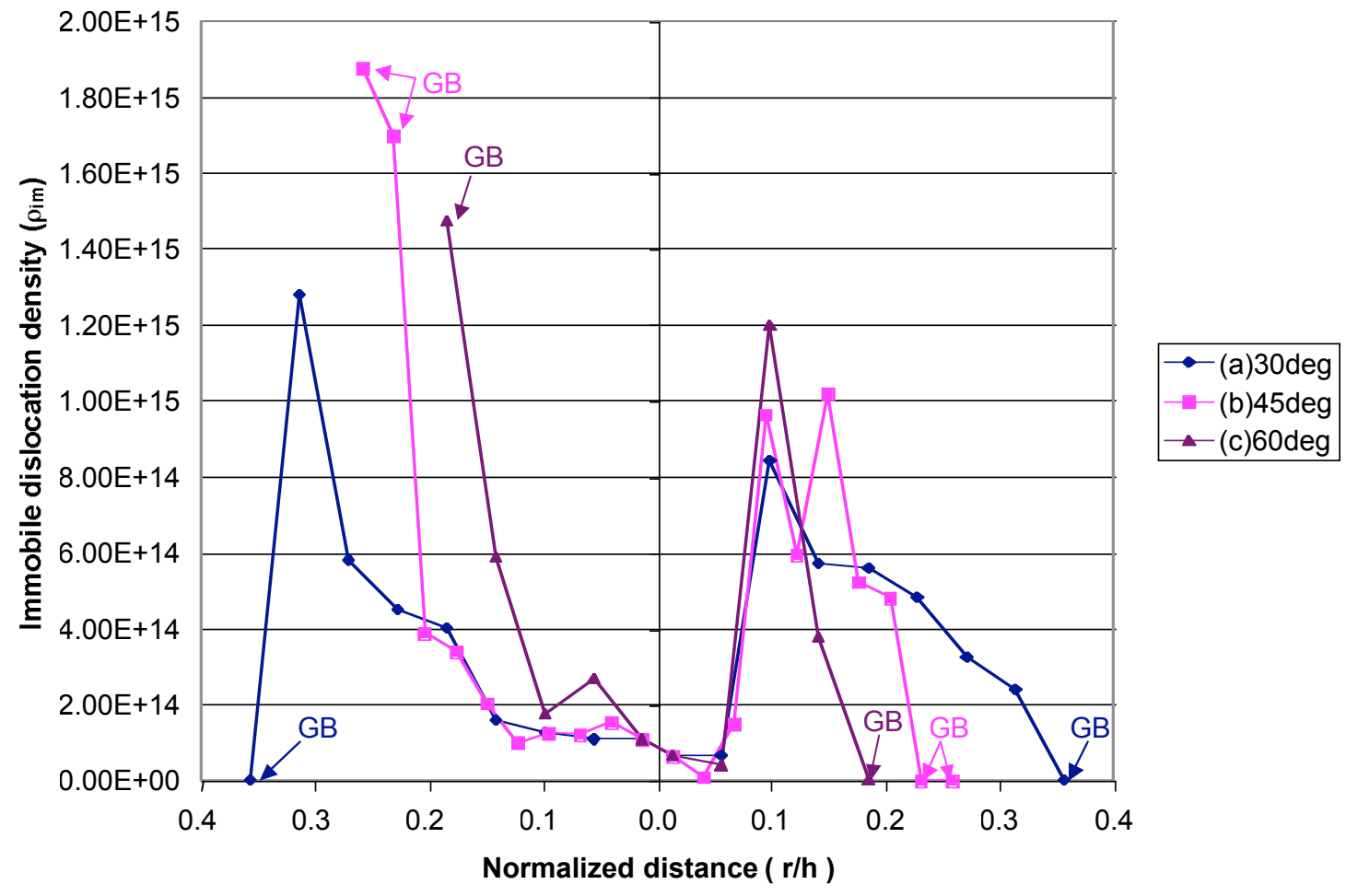

(iii)

Figure 7.12. Immobile dislocation-density distribution along the different angles with respect to loading axis at final loading -15 grains and low angle GB orientation;

(a) $\pm 30^{\circ}(\mathrm{GB}: \mathrm{r} / \mathrm{h}= \pm 0.3569)$, (b) $\pm 45^{\circ}$ (GB: $\mathrm{r} / \mathrm{h}=0.2312$ and 0.2584$)$, (c) $\pm 60^{\circ}$ (GB: $\mathrm{r} / \mathrm{h}=0.185$ ) (i) $9 \%$, (ii) $12.8 \%$, and (iii) $23.1 \%$ 


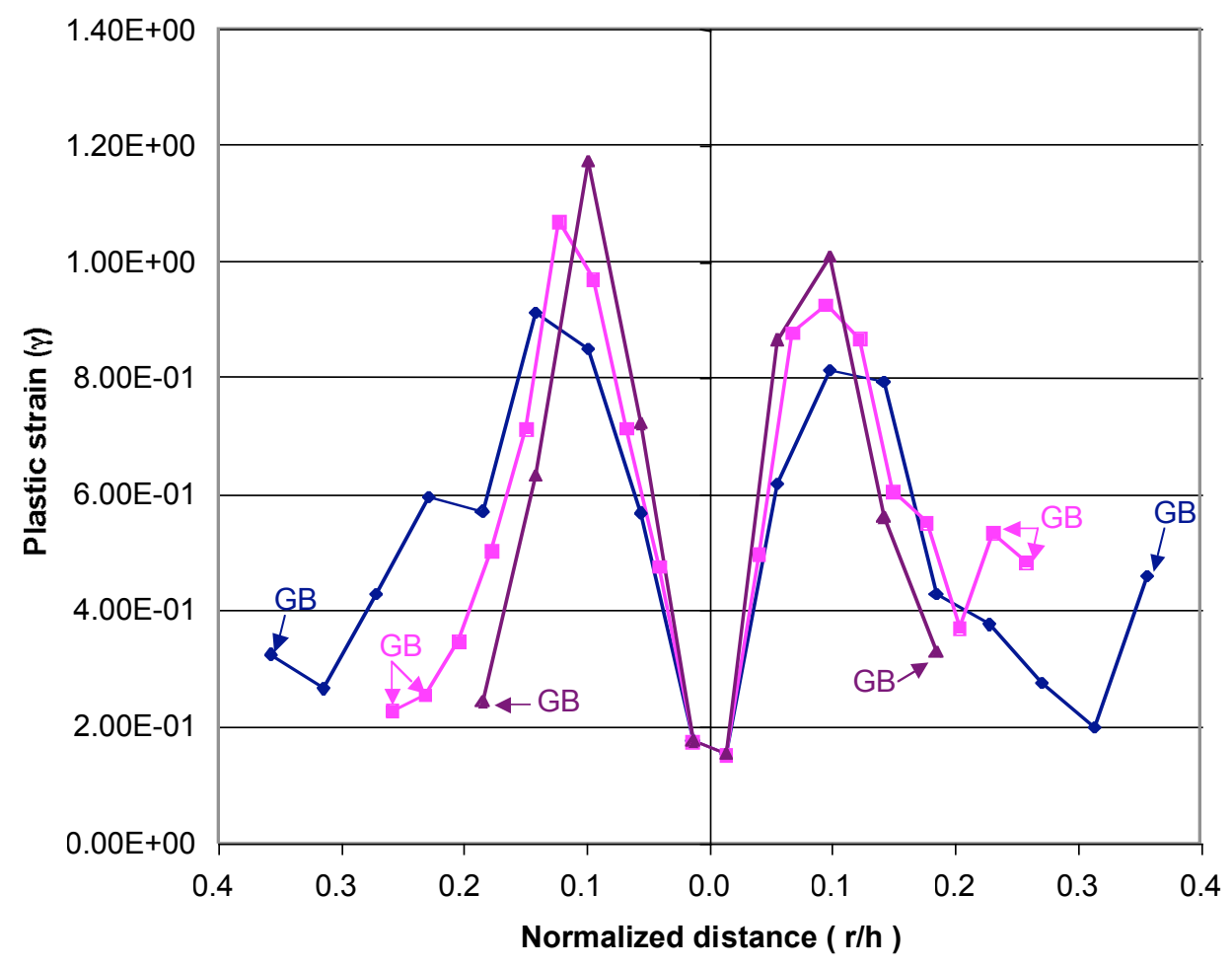

- (a)30deg
- (b)45deg
- (c)60deg

(i)

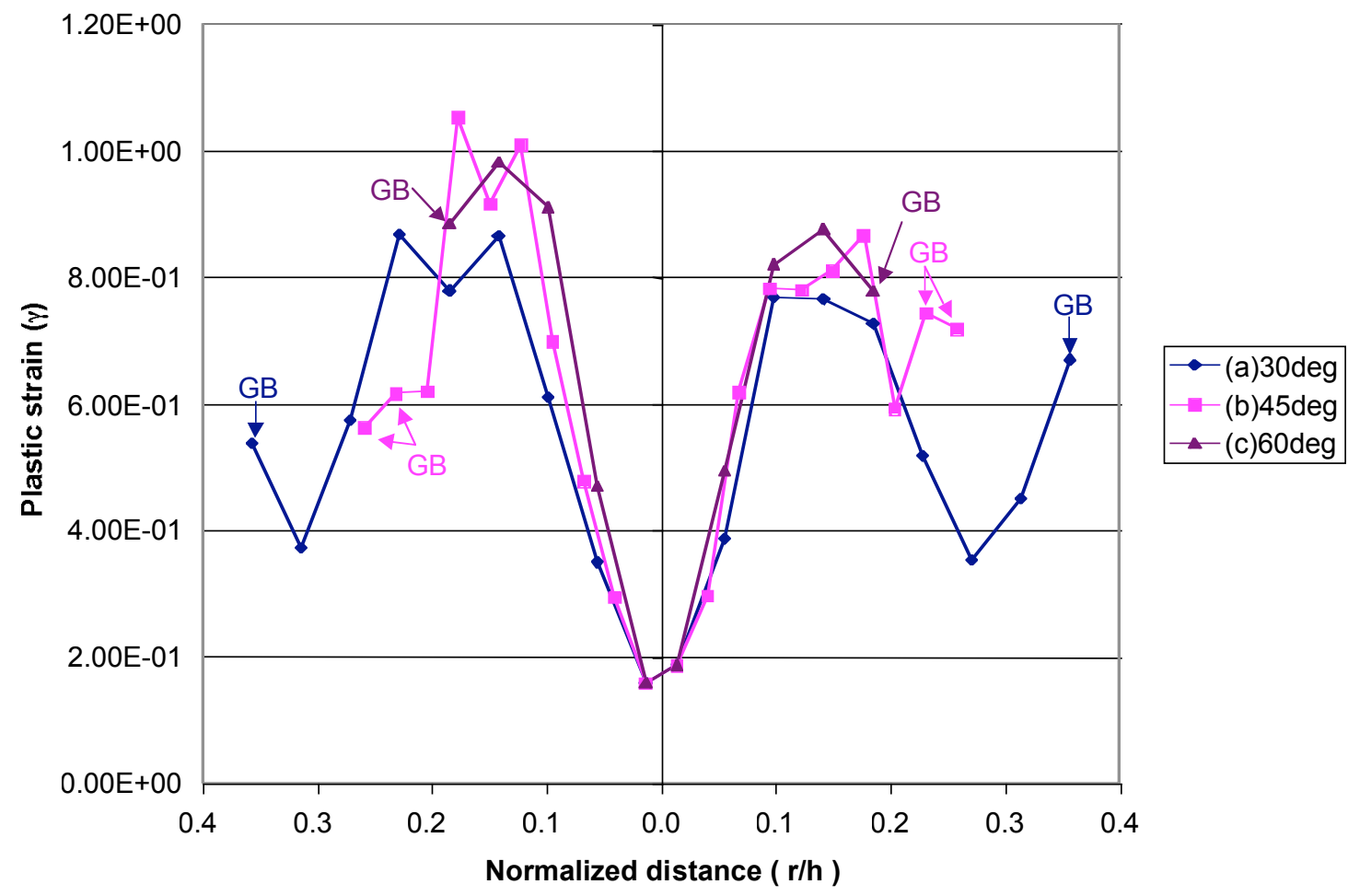

(ii) 


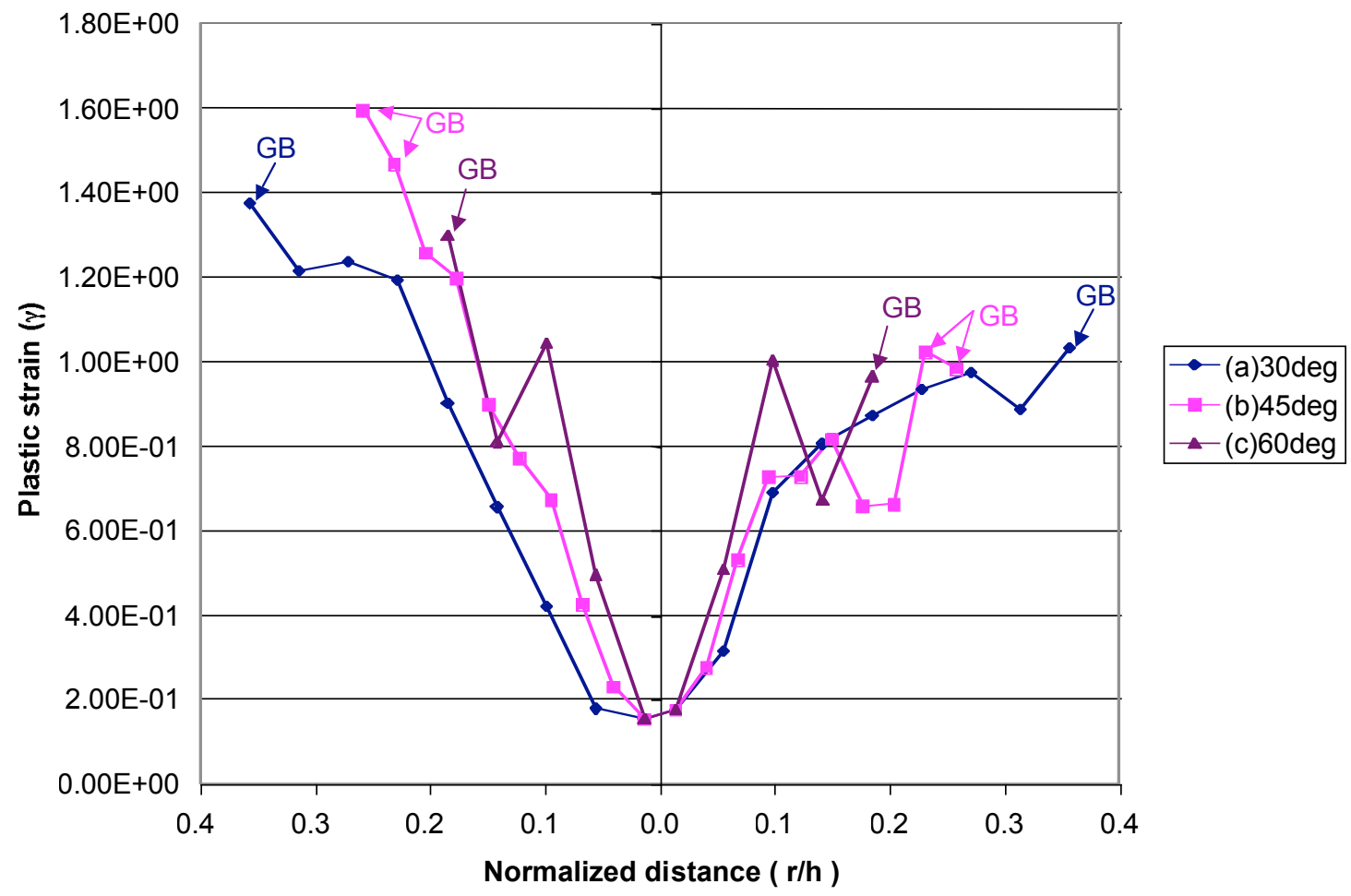

(iii)

Figure 7.13. Plastic strain distribution along the different angles with respect to loading axis at final loading - 15 grains and low angle GB orientation;

(a) $\pm 30^{\circ}(\mathrm{GB}: \mathrm{r} / \mathrm{h}=0.3569)$, (b) $\pm 45^{\circ}$ (GB: $\mathrm{r} / \mathrm{h}=0.2312$ and 0.2584$)$, (c) $\pm 60^{\circ}(\mathrm{GB}: \mathrm{r} / \mathrm{h}=0.185$ ) (i) $9 \%$, (ii) $12.8 \%$, and (iii) $23.1 \%$ 


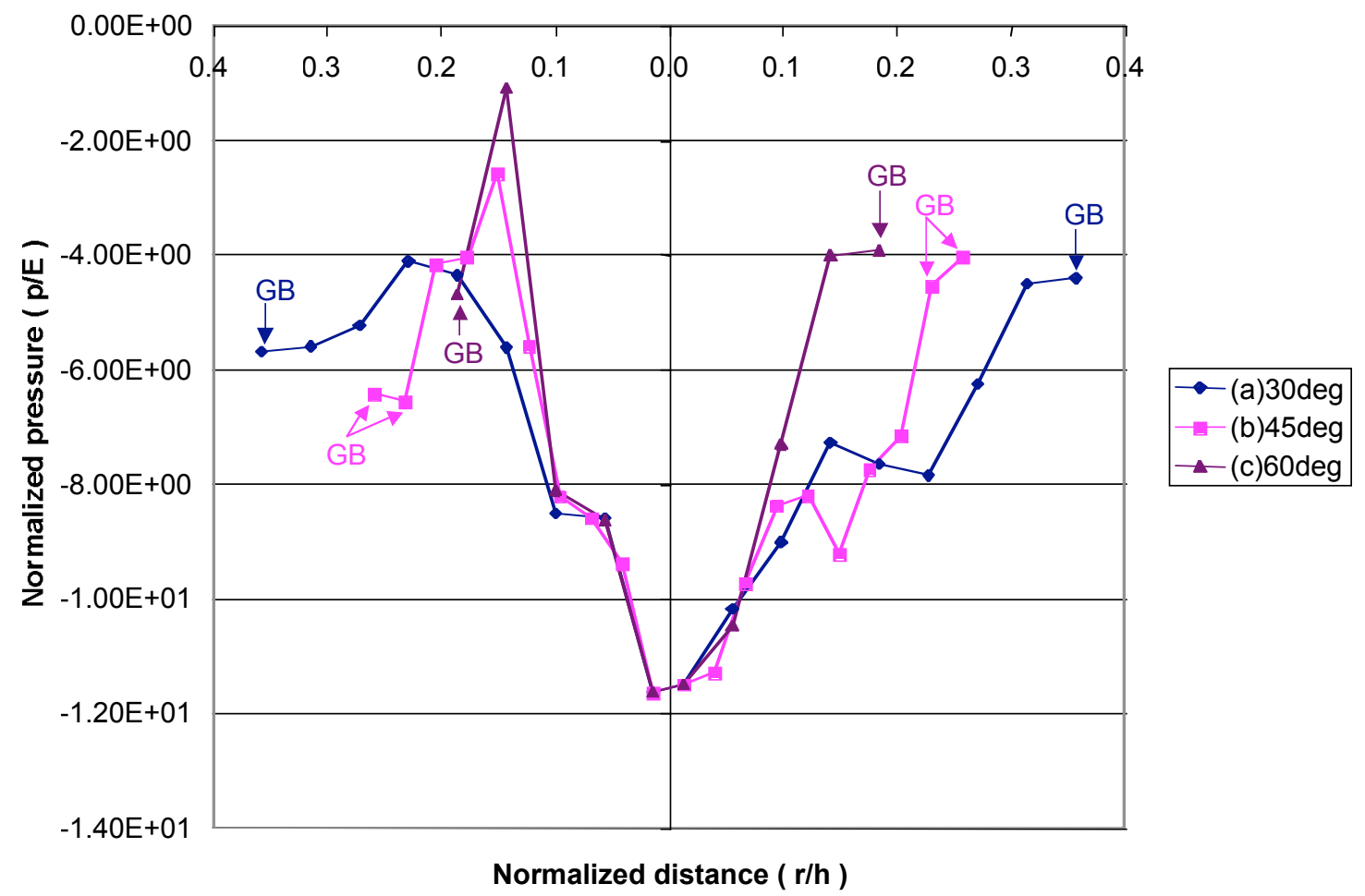

(i)

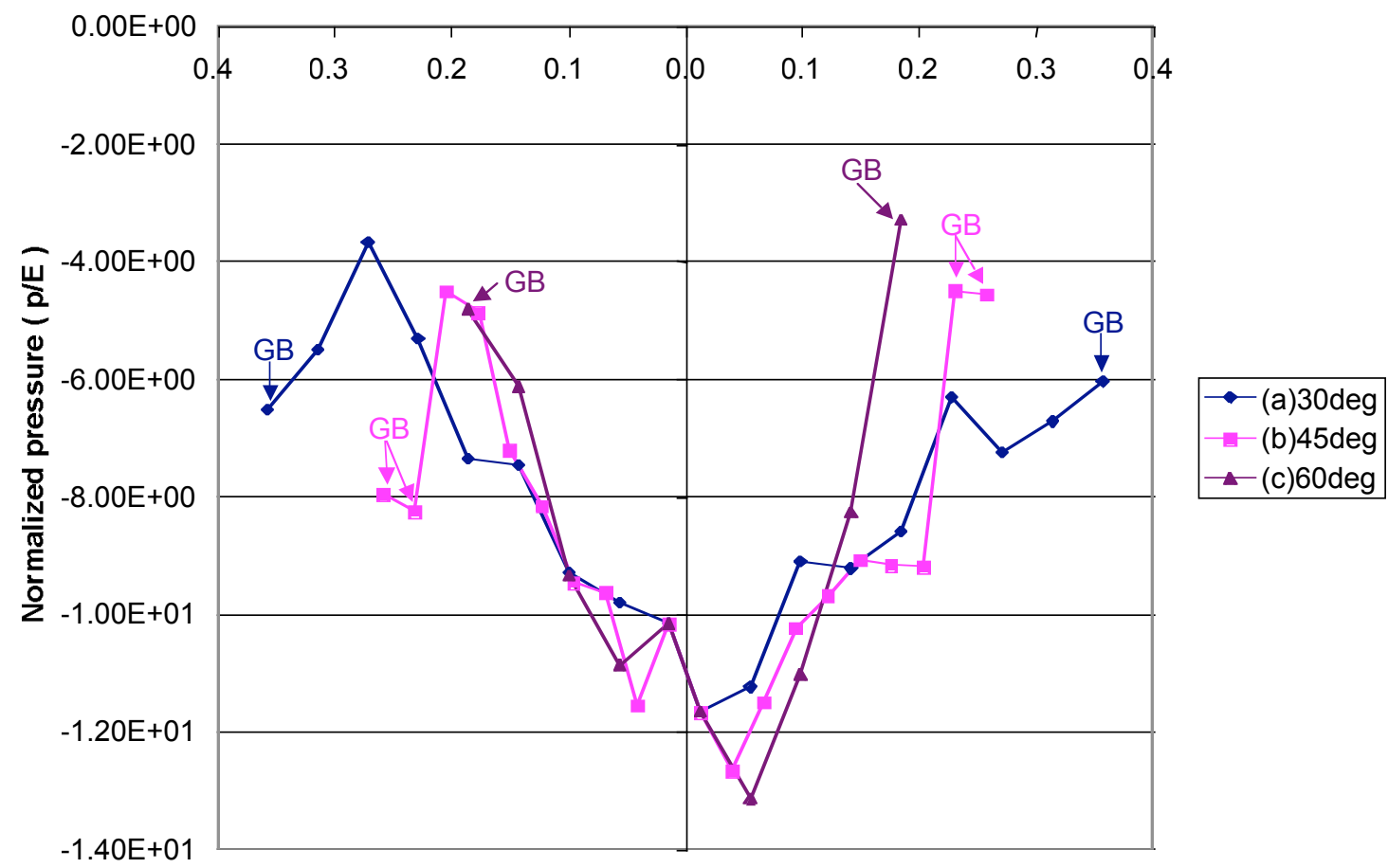

Normalized distance ( $\mathrm{r} / \mathrm{h}$ )

(ii) 


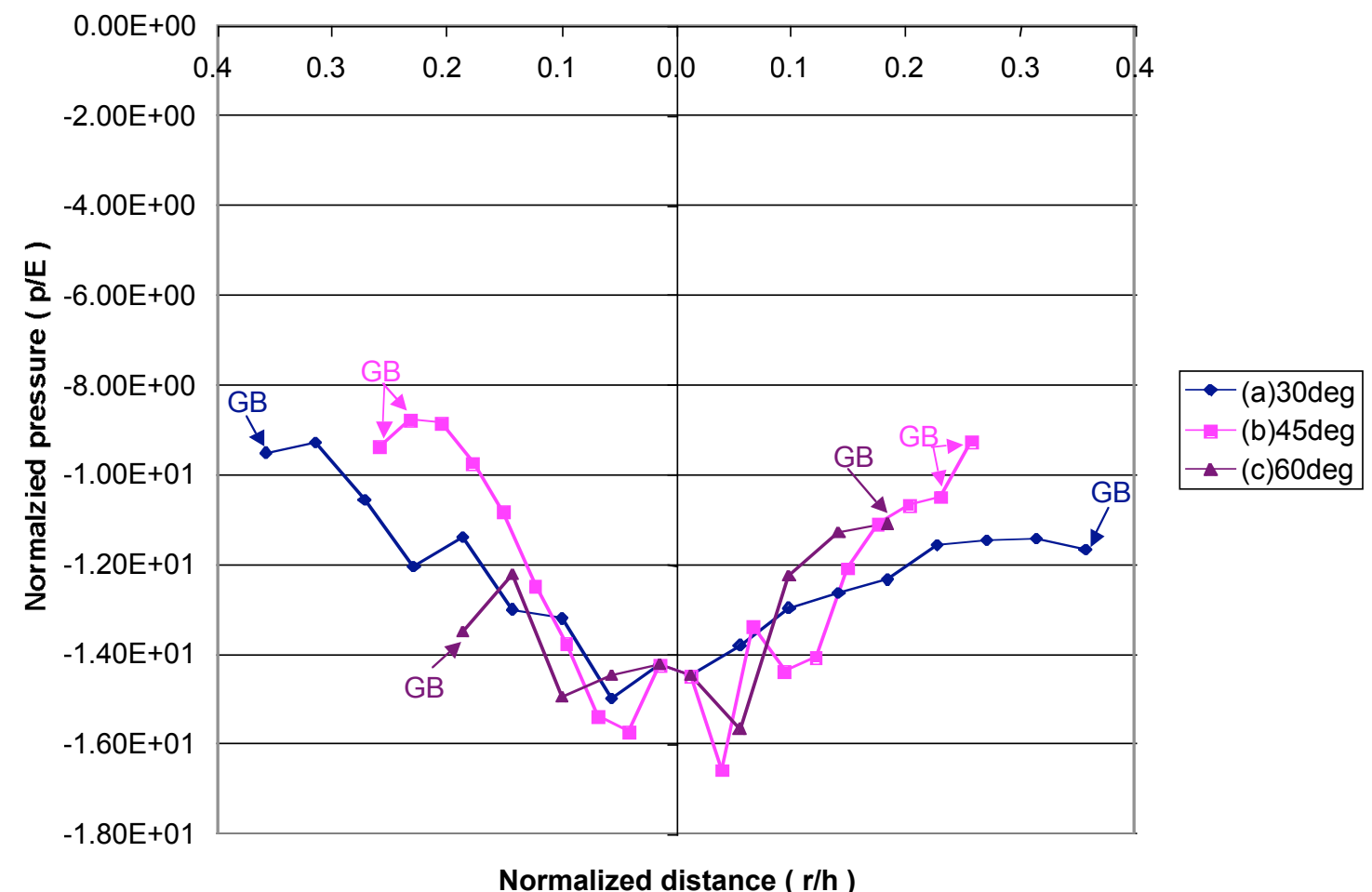

(iii)

Figure 7.14. Normalized pressure distribution along the different angles with respect to loading axis at final loading - 15 grains and low angle GB orientation;

(a) $\pm 30^{\circ}$ (GB: $\left.\mathrm{r} / \mathrm{h}=0.3569\right)$, (b) $\pm 45^{\circ}$ (GB: $\mathrm{r} / \mathrm{h}=0.2312$ and 0.2584 ), (c) $\pm 60^{\circ}(\mathrm{GB}: \mathrm{r} / \mathrm{h}=0.185$ ) (i) $9 \%$, (ii) $12.8 \%$, and (iii) $23.1 \%$ 

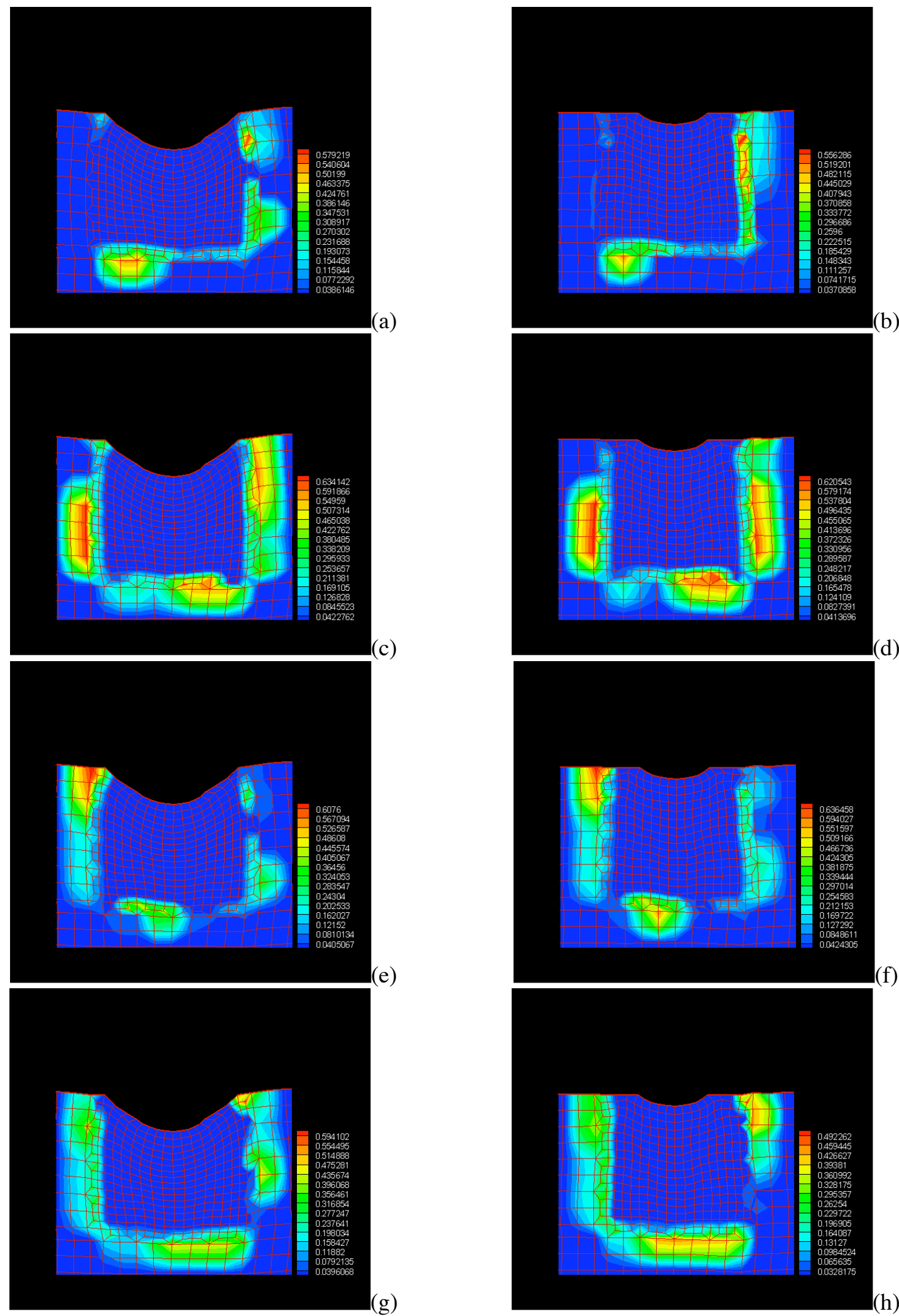

Figure 7.15. Transmission factor at GBs under the virtual indenter: Model 1 with $9 \%$ loading

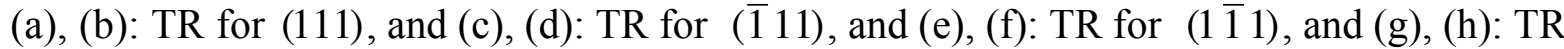
for $(11 \overline{1})$ 


\section{CHAPTER 8}

\section{RANDOM HIGH ANGLE GB EFFECTS ON NANOINDENTATION OF POLYCRYSTALLINE AGGREGATES}

In chapter 7 we presented results for random low angle GB misorientation distribution for polycrystalline aggregates. We will investigate the nanoindentation of aggregates with high angle misorientations of grains and GBs, where the misorientations do not exceed $30^{\circ}$. We will also investigate how nanoindentation is affected by these high angle GBs. We will analyze the effects of grain and aggregate size and how GB strengths affect deformation modes induced by nanoindenation. The strength of the GB will be modified in the same way as done by Ashmawi and Zikry (2002) where the initial GB static yield strength and modulus were assumed to three times higher than nearby grains.

\subsection{Grain-size Effects and Slip-system Activities during Local Unloading}

Fifteen-grains and twenty eight-grains polycrystalline aggregates with random high angle grain and GB orientations were investigated. For different grain-size, $0.003 \mathrm{~mm}$ (Model 1, 15 grains) and $0.0022 \mathrm{~mm}$ (Model 2, 28 grains), 9\% and 12.8\% indentation depths were pursued. Figure 8.1 represented the global stress-strain curves of these four cases. Increasing the compressive nominal strains match increasing the average stresses. The larger grain-sized aggregate has larger nominal strains than low angle models during unloading and

for both models more strains but less stress values were shown compare to the single crystal models. 
Slip-system activity changes of the stress-strain curve during the local unloading were shown in Table 8.1. One but different slip-system was most active before and after the load-drop in polycrystalline aggregates with high angle GB models. For Model 1 at a normalized depth of $9 \%$ and $12.8 \%$, slip-system 4 was most active before and after the local load-drops. However, the most active slip-system was varied for different slip-systems before and after the load-drop in Model 2 as in Table 8.1. From this investigation we found that smaller grain-sized model (Model 2) had the potential slip-system activity variation during the local unloading steps. This is due to lattice rotations and plastic strain accumulation and pressures in indented grain, which is experimentally observed by Michalske and Houston (1998). We also found that the load-drop during the indentation of polycrystalline aggregates affects the dislocation slip-system activities, and this was similar result as in low angle misorientation models but for different slip-systems.

Table 8.1. Slip-system activity variation before and after the load-drops during global loading (high angle GB misorientation)

\begin{tabular}{ccc}
\hline Normalized Indented Depth & Before load-drop & After load-drop \\
\hline $9 \%$ Model 1 & 4 & 4 \\
12.8\% Model 1 & 4 & 4 \\
$9 \%$ Model 2 & 11 & 3 \\
$12.8 \%$ Model 2 & 3 & 10 \\
\hline
\end{tabular}

\subsection{Immobile Dislocation-density Evolution, Plastic Strain, and Pressure Response and Effects of Increased Depths and Aggregate Sizes: Model 1 and Model 2}

Most active slip-systems at the final loading step at different normalized depths were investigated to determine how the immobile dislocation-densities on these systems evolve 
and its summary is shown in Table 8.2. Different slip-systems are most active at each model and loading conditions.

Table 8.2. List of most active slip-systems at maximum normalized depths

\begin{tabular}{cccc}
\hline $\begin{array}{c}\text { Normalized } \\
\text { Indented Depth }\end{array}$ & Most active slip-systems & $\begin{array}{c}\text { Normalized } \\
\text { Indented Depth }\end{array}$ & Most active slip-systems \\
\hline $9 \%$ Model 1 & 4 & $9 \%$ Model 2 & 11 \\
$12.8 \%$ Model 1 & 5 & $12.8 \%$ Model 2 & 10 \\
\hline
\end{tabular}

By investigating material behavior at final the loading and unloading, we knew that slip-system 4 was the most active slip-system at the final loading step for $9 \%$ indentation of Model 1. Immobile dislocation-density evolved to $4.58 \times 10^{15} \mathrm{~m}^{-2}, 46 \%$ of the saturation value (Figure 8.2a). The total immobile dislocation-density for all slip-systems had evolved to $9.56 \times 10^{15} \mathrm{~m}^{-2}, 96 \%$ of the saturation value (Figure $8.2 \mathrm{~b}$ ). Comparing to the low angle models, significantly low immobile dislocation-density was evolved for most active system. Hence, the immobile dislocation-density of the most active slip-system accounts for $48 \%$ of all immobile dislocation-density activities comparing to $85 \%$ in low angle model with same normalized depth. It might be caused by the slip-system activity blockage due to highly misoriented distribution of Euler angles between grains and GBs. The immobile dislocationdensity at final global unloading of slip-system $4\left(4.69 \times 10^{16} \mathrm{~m}^{-2}\right)$ and total immobile dislocation-density $\left(8.49 \times 10^{16} \mathrm{~m}^{-2}\right)$ at the same step are shown in Figures $8.2 \mathrm{c}-\mathrm{d}$. These are very close to same indentation depth model for low angle misorientation. This indicates that more dislocation-density evolved in unloading steps than the loading steps for high angle model. After the unloading, this system accounts for $55.3 \%$ of total immobile dislocationdensity evolution. Similar to single crystals models, immobile dislocation-density continues 
to evolve during the unloading step. Highly localized distribution of the immobile dislocation-density was also evident at either side of the indenter for the most active slipsystem in both final loading and unloading steps. This indicates that different slip-systems are activated during unloading, and this is related to the residual strains due to inelastic activity.

The accumulated plastic strains and pressure distributions at the final loading and unloading step are shown in Figure 8.3. Smaller plastic strains are accumulated (1.79) than low angle models (2.26) in the regions corresponding to the highest immobile dislocationdensity areas at the final loading. The highest negative normalized pressures at the final loading step locate at the same regions, with a value of -18.52 .

For further study we also indented the model 1 to the normalized depth of $12.8 \%$. Result plots are shown in Figure 8.4 and Figure 8.5. At a normalized depth of $12.8 \%$, slipsystem 5 had the highest immobile dislocation-density evolution of $7.12 \times 10^{15} \mathrm{~m}^{-2}$ at final loading step, which is $71 \%$ of the saturation value. (Figure $8.4 \mathrm{a}$ ) The total immobile dislocation-density evolved to $1.59 \times 10^{16} \mathrm{~m}^{-2}$ (Figure 8.4b). At unloading, the most active slip-system had an immobile dislocation-density of $2.73 \times 10^{16} \mathrm{~m}^{-2}$ (Figure $8.4 \mathrm{c}$ ), and total immobile dislocation-density had $1.05 \times 10^{17} \mathrm{~m}^{-2}$ (Figure $8.4 \mathrm{~d}$ ). The portion of active systems at final loading is $44 \%$. However, it decreases to $27 \%$ in unloading step. This means that active systems evolution portion of other systems is tremendously increased on unloading stages. Hence, as the normalized depth increases, and more grains are indented, the residual strains in the deformed grains result in more slip-systems activation. This is consistent with experimental observations (see, for example, Choi, Van Vliet et al., 2003), which indicate that dislocations and strains accumulate within the specimen after unloading. The 
accumulated plastic strains are also increased about 13\% (1.98), Figure 8.5a, from the 9\% indentation model at the final loading step. The pressures attain the highest negative normalized value of -18.1 at the maximum loading point (Figure $8.5 \mathrm{c}$ ).

We investigated smaller grain-size effects for two normalized indentation depths (Figures 8.6-8.9). For 9\% normalized indentation depth, slip-system 11 was the most active at the final loading step. The immobile dislocation-density had $3.25 \times 10^{15} \mathrm{~m}^{-2}, 33 \%$ of the saturation (Figure $8.6 \mathrm{a}$ ) and only $6.86 \times 10^{15} \mathrm{~m}^{-2}, 69 \%$ of the saturation, was evolved for total immobile dislocation-density (Figure 8.6b). At the final unloading stages, immobile dislocation-density was evolved to $1.97 \times 10^{16} \mathrm{~m}^{-2}$ for the most active slip-system and $4.47 \times 10^{16} \mathrm{~m}^{-2}$ for total immobile dislocation-density (Figures 8.6c-d). The immobile dislocation-density had $2.28 \times 10^{16} \mathrm{~m}^{-2}$ at the final loading (Figure $8.8 \mathrm{a}$ ), $1.15 \times 10^{17} \mathrm{~m}^{-2}$ for final unloading for active system 10 (Figure 8.8c), and the total immobile dislocationdensity had evolved to $2.72 \times 10^{16} \mathrm{~m}^{-2}$ at the final loading (Figure $8.8 \mathrm{~b}$ ), and $2.66 \times 10^{17} \mathrm{~m}^{-2}$ at final unloading (Figure 8.8d). Both the accumulated plastic strains and pressures were continuously increased for this model (Figure 8.7 and Figure 8.9). The high immobile dislocation-densities were accumulated at the regions, which had highest pressures during the loading and unloading.

\subsection{Effects of High Strength GBs on Nanoindentation}

In this section we investigated the results of the stronger GB models, which have the three times larger initial GB static yield strength than surrounding grains, for fifteen and twenty-eight grains with shallow indenter: $9 \%$ normalized indentation depth. 
Model 3 has large grain-size $(0.003 \mathrm{~mm})$, and 9\% indentation depth was applied. Slip-system 1 was the most active slip-system at the final loading step and had immobile dislocation-density of $4.98 \times 10^{15} \mathrm{~m}^{-2}, 50 \%$ of the saturation value. The total immobile dislocation-density evolved to $1.12 \times 10^{16} \mathrm{~m}^{-2}$ at the final loading stage. At the final unloading, the most active slip-system 1 had $3.44 \times 10^{16} \mathrm{~m}^{-2}$ and total immobile dislocation-density had $9.1 \times 10^{16} \mathrm{~m}^{-2}$ (Figure 8.10). At the final loading step, immobile dislocation-density of the most active slip-system accounts for $45 \%$ and at final unloading step, it accounts for $37.4 \%$ of total immobile dislocation-dislocation evolution. This was less portion of the most active slip-system comparing to the Model 1 . However, dislocation-density was more evolved than Model 1 at loading and unloading steps. This indicates that huge evolution of other slipsystems than most active system during loading and unloading steps. Same properties except smaller grain-size $(0.0022 \mathrm{~mm})$ were defined as Model 4 . The immobile dislocation-density had $6.70 \times 10^{15} \mathrm{~m}^{-2}, 67 \%$ of saturation value at the final loading step for most active slipsystem 3. And the total immobile dislocation-density had $1.0 \times 10^{16} \mathrm{~m}^{-2}$ at the final loading step. At final unloading, system 3 evolved to $2.3 \times 10^{16} \mathrm{~m}^{-2}$. And for the same step, total immobile dislocation-density had $5.8 \times 10^{16} \mathrm{~m}^{-2}$ (Figure 8.12 ). Similar to model 3 , model 4 showed more dislocation-density evolution than model 2. From these results we investigated that immobile dislocation-density evolved more actively in models, which have stronger GB initial static yield strength than models that have same initial GB yield strength. 


\subsection{Crystalline Orientation Effects}

We investigated for the grain under the indenter and adjacent GBs to figure out the influences of the indentation and their spatial and angular variation of immobile dislocationdensities, plastic-strains, and pressures on that grain and GBs for same angular orientations and distances as we discussed in chapter 7. We could investigate grain and GBs behavior at critical locations and orientations. Figure 8.14 shows the immobile dislocation-density variation of Model 1 at different normalized indentation depths. The immobile dislocationdensity values varied along the angular directions and they are highest under the indenter. However, for $12.8 \%$ model, the immobile dislocation-density increased at the left side of the indenter near the specimen surface and GBs, which coincides with the increase of pressures and plastic strains for same regions. Plastic strains and pressures are shown in Figure 8.15 and Figure 8.16. We investigate that near the GBs, there exists increase of the local plastic strains and pressures between orientations of $30^{\circ}$ and $45^{\circ}$, which is near the maximum shear stress orientations of $45^{\circ}$. These are consistent with the observations by Ohmura, Minor et al. 2004, which concluded that the GB and orientations are the principal mechanisms on nanoindentation experiments.

\subsection{GB Transmission Factors}

Figure 8.17 represented the calculated transmission factors (TR) contour plots around the GBs at a normalized indentation depth of 9\%, for Model 1. Plots c and d, which included the most active slip-system 4 showed the highest immobile dislocation-density 
transmission at the right hand side, which had highest pressures. With these calculations, we knew that the hydrostatic pressures are a main factor for dislocation-density activity which is supported with the MD observations of Feichtinger, Derlet et al. (2003), related to the critical role that pressures play in dislocation emission and absorption near GBs.

\subsection{Summary and Conclusions}

This study provides an extensive understanding of the interrelated physical mechanisms that result in material behavior due to nanoindentation in polycrystalline aggregates with high angle misorientation distributions for grains and GBs. A multiple-slip rate-dependent crystalline constitutive formulation that is coupled to the evolution of mobile and immobile dislocation-densities and the hierarchical computational schemes has been developed to investigate microstructually-induced plasticity due to the nanoindentation of

f.c.c. gold aggregates which has the same initial mobile and immobile dislocation-density of both grains and GBs, and the initial yield stress on GB is either the same as the bulk grains or three-times stronger than the bulk grains. The hierarchical model was used to scale the MD displacement profiles to the micro level. High angle GB effects were accounted for by using random high angle misorientations for different sized aggregates and grains.

These high angle GB misorienations resulted in significantly different behavior than the cube-oriented aggregates. The predictions were used to determine how at local unloading, there was slip-system activation, at different indentation depths. There was no slip-system activity change during the short unloading period for larger grain-size with high angle GB distributions. However, similar to the low angle distributions, there was slip-system variation 
for smaller grain-size models. For larger grain-size models, dislocation-density evolved less than low angle GB distribution model. It may be caused by the highly misoriented Euler angles between grains and GBs to obstruct slip movement of dislocation-density. These results match with less accumulation of inelastic strains during the loading and unloading steps. In smaller grain-size models, however, both dislocation-density evolution and accumulation of plastic strains had very similar values to low angle models due to reduced grain-sizes. And these dislocation-density evolutions also match well with the results of plastic strains accumulation and pressure distributions. Furthermore, it was shown how the immobile dislocation-densities evolve at the maximum loading and unloading points, and how the nominal residual strains are intricately related to the accumulation of plastic strains and pressures. We were also able to identify the exact locations and orientations of the immobile dislocation-densities, plastic strains, and pressures from the indentation surface. These values generally decreased, as the normalized distance from the indenter contact point increased. At the maximum indentation point, there was a sharp increase of immobile dislocation-density and plastic strains near specific GBs and surface areas. This can be due by the bending effects associated with larger indentation depth (see, for example, Zimmerman, Kelchner et al., 2001; Knap and Ortiz, 2003; Greer and Nix, 2005). Knowing that the GBs at specific locations played a dominant role in immobile dislocation-density evolution and slip-system switching, we used the GB interfacial scheme to determine exactly where and how immobile dislocation-densities transmitted or where impeded. Our predictions indicate that the high GB transmission rates are directly related to the high pressures near the GB regions. Hence, we could relate how local pressures at specific GB 
locations and orientations affect immobile dislocation-density evolution during nanoindentation.

The higher strength GBs had higher dislocation-density evolution and higher plastic strains in comparison with the same strength GBs distributions. This is due to the stiffer GB, which may have similar function of higher misorientation angles, and may block the transmission of the dislocation movement. Decreasing values on investigation of angular variation with respect to loading axis was also shown. By using the interfacial GB kinematic model, we were able to identify the exact slip-system in the GB region corresponding to the most active slip-system. And the calculated transmission factors for the GBs also indicate that the most active slip-system at the final loading step coincided with the highest dislocation-density evolution.

This study underscores how the hierarchical computational scheme, the dislocationdensity based formulation, and the GB interfacial kinematics can provide a comprehensive understanding of the effects of multiple-slip inelastic deformation and dislocation interactions, which are associated with nanoindenation in polycrystalline aggregates with high angle GB misorientations. Critical GB and grain locations can be identified and deformation modes can be tracked, and hence these predictions can be used to obtain desired microstructural behavior. 


\section{References}

Ashmawi, W. M. and M. A. Zikry (2002). "Prediction of grain-boundary interfacial mechanisms in polycrystalline materials." Journal of Engineering Materials and Technology-Transactions of the Asme 124(1): 88-96.

Choi, Y., K. J. Van Vliet, et al. (2003). "Size effects on the onset of plastic deformation during nanoindentation of thin films and patterned lines." Journal of Applied Physics 94(9): 6050-6058.

Feichtinger, D., P. M. Derlet, et al. (2003). "Atomistic simulations of spherical indentations in nanocrystalline gold." Physical Review B 67(2).

Greer, J. R. and W. D. Nix (2005). "Size dependence of mechanical properties of gold at the sub-micron scale." Applied Physics a-Materials Science \& Processing 80(8): 16251629.

Knap, J. and M. Ortiz (2003). "Effect of indenter-radius size on Au(001) nanoindentation." Physical Review Letters 90(22).

Michalske, T. A. and J. E. Houston (1998). "Dislocation nucleation at nano-scale mechanical contacts." Acta Materialia 46(2): 391-396.

Ohmura, T., A. M. Minor, et al. (2004). "Dislocation-grain boundary interactions in martensitic steel observed through in situ nanoindentation in a transmission electron microscope." Journal of Materials Research 19(12): 3626-3632.

Zimmerman, J. A., C. L. Kelchner, et al. (2001). "Surface step effects on nanoindentation." Physical Review Letters 8716(16): art. no.-165507. 


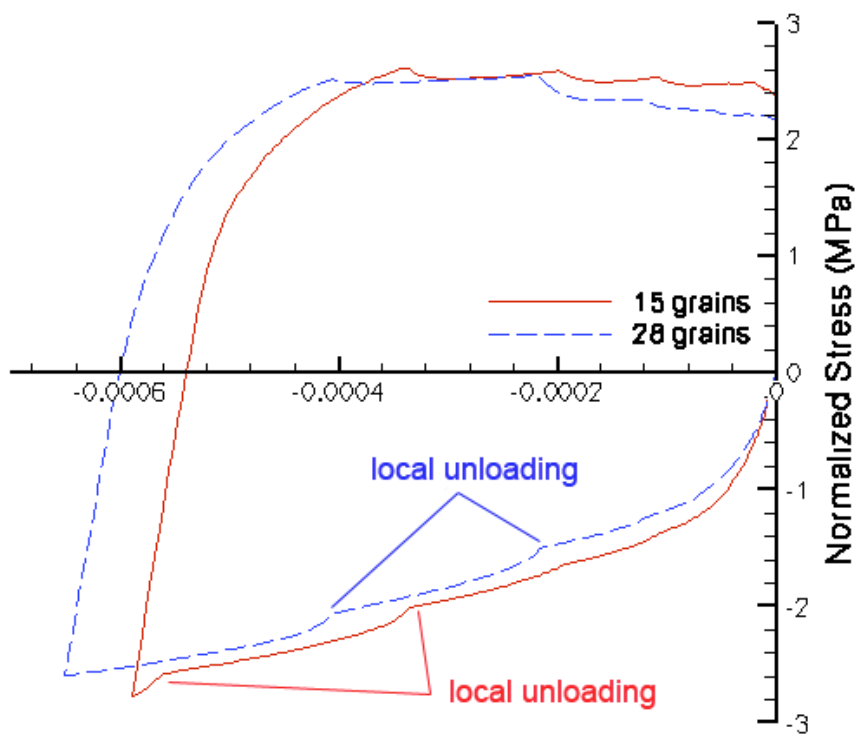

Nomınal Straın

(a)

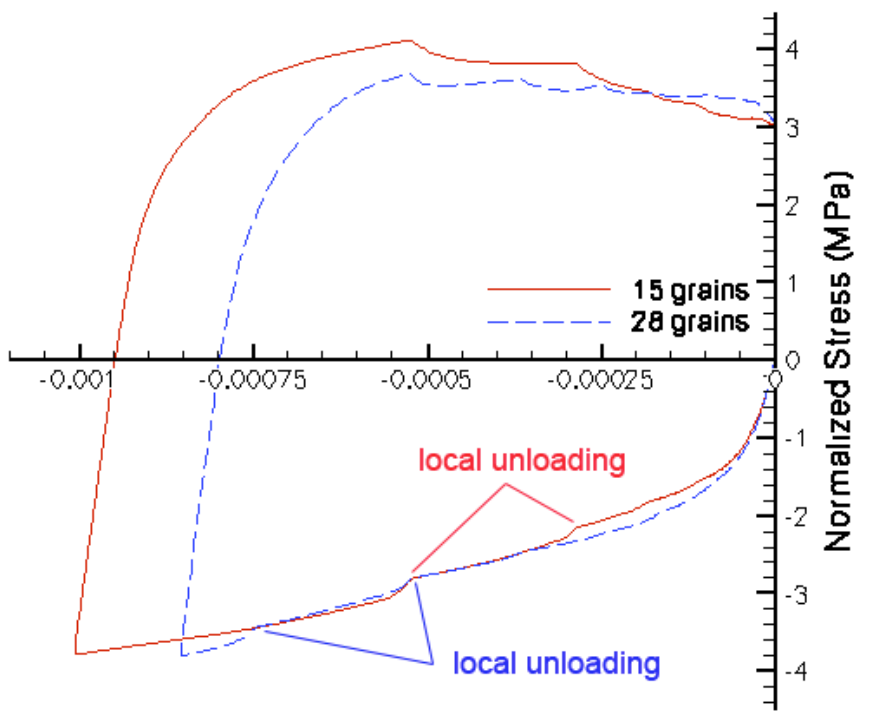

Nomınal Straın

(b)

Figure 8.1. Stress-strain curves for gold polycrystalline aggregates with different normalized indented depths, aggregate sizes, and high angle misorientations (a) $9 \%$ (b) $12.8 \%$ 


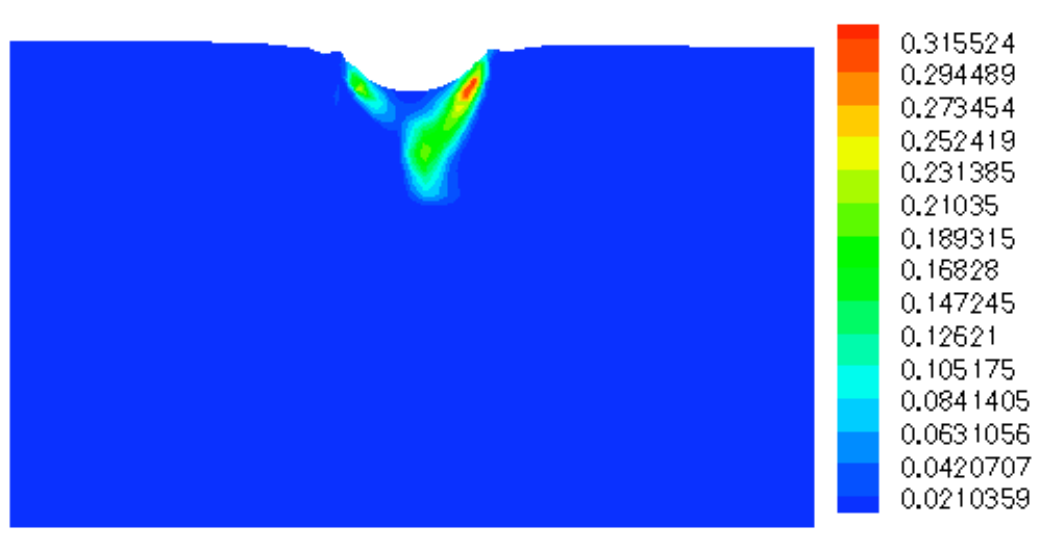

(a)

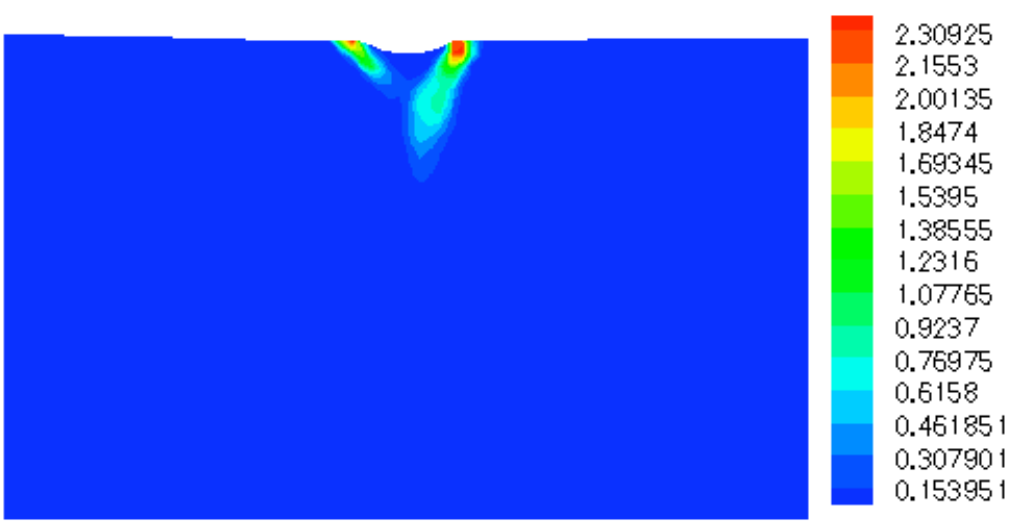

(c)

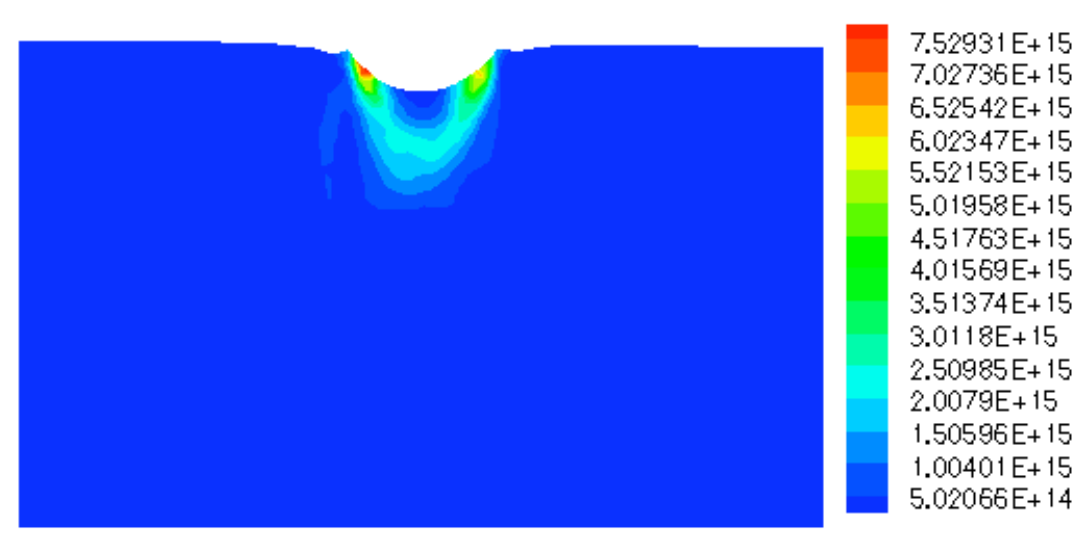

(b)

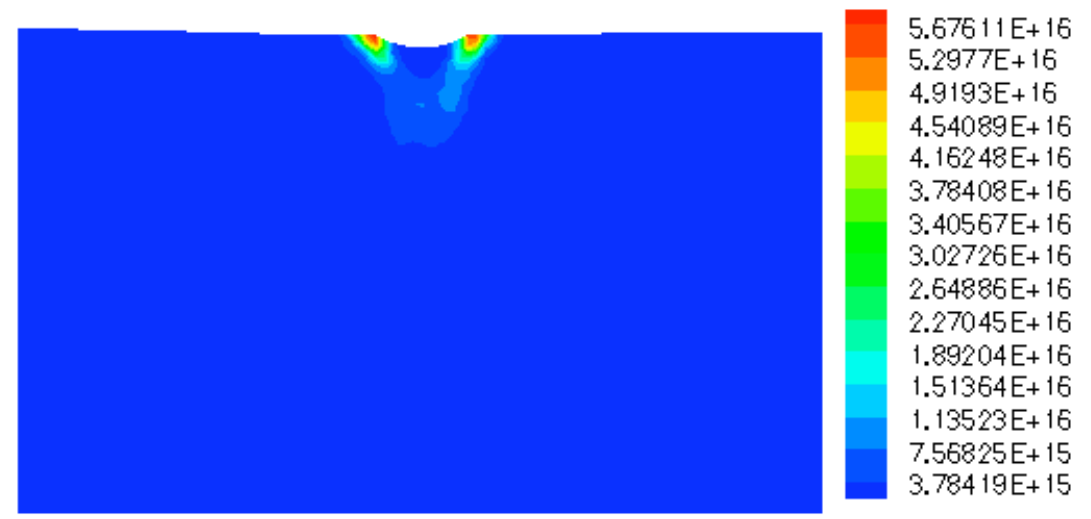

(d)

Figure 8.2. Immobile dislocation-density at final loading step for system 4 normalized by saturation (a), total immobile dislocationdensity at final loading step non-normalized (b), just before unloading step for system 4 normalized by saturation (c), and total immobile dislocation-density just before unloading step non-normalized (d). (Model 1 with 9\% indentation) 


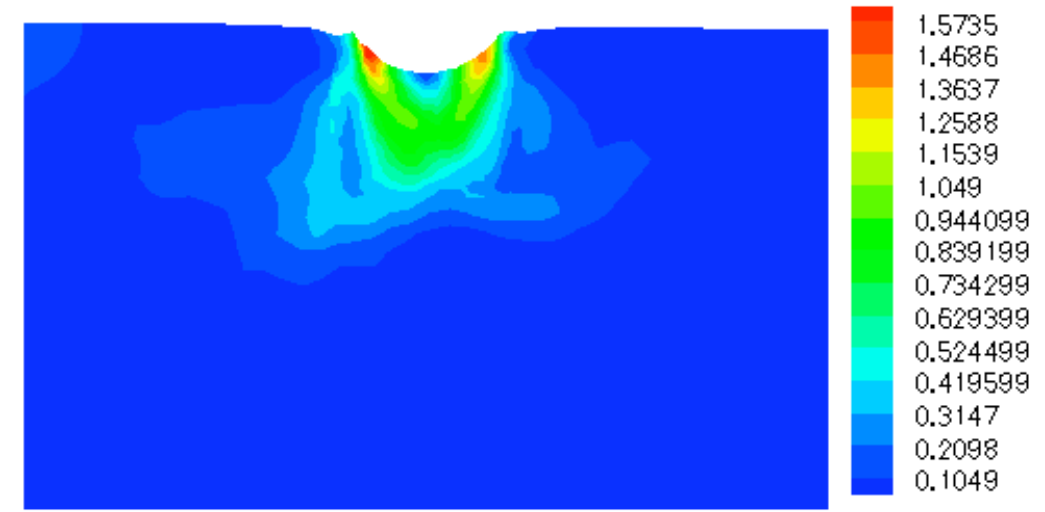

(a)

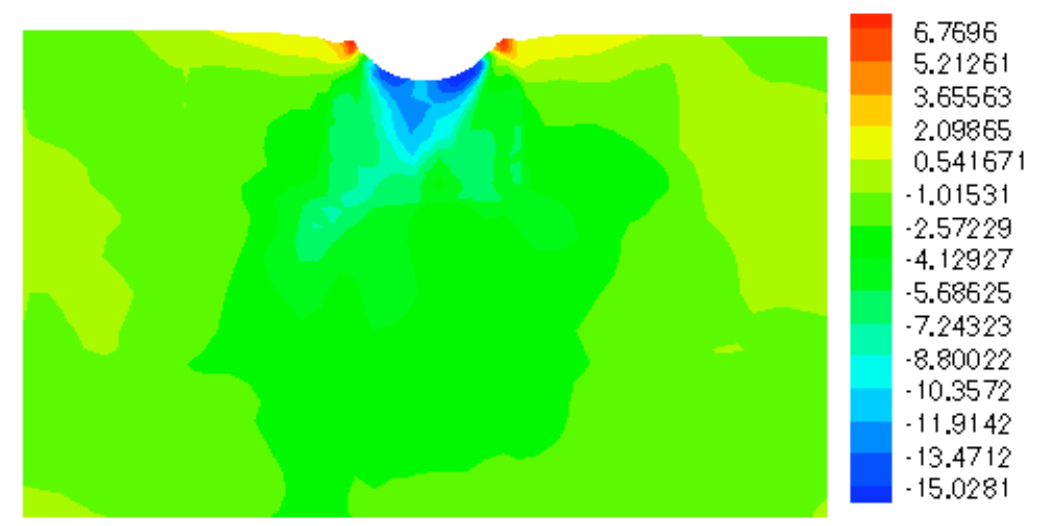

(c)

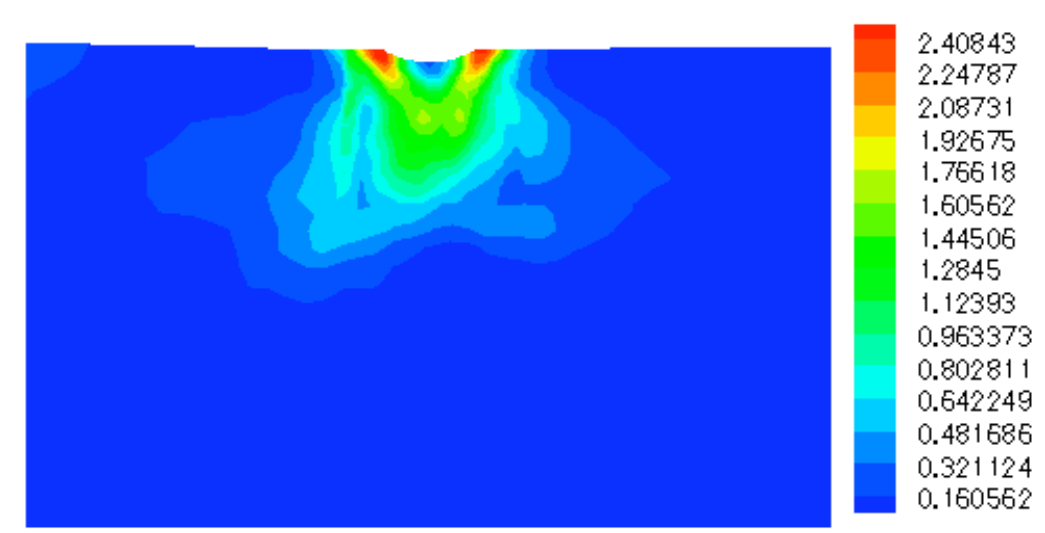

(b)

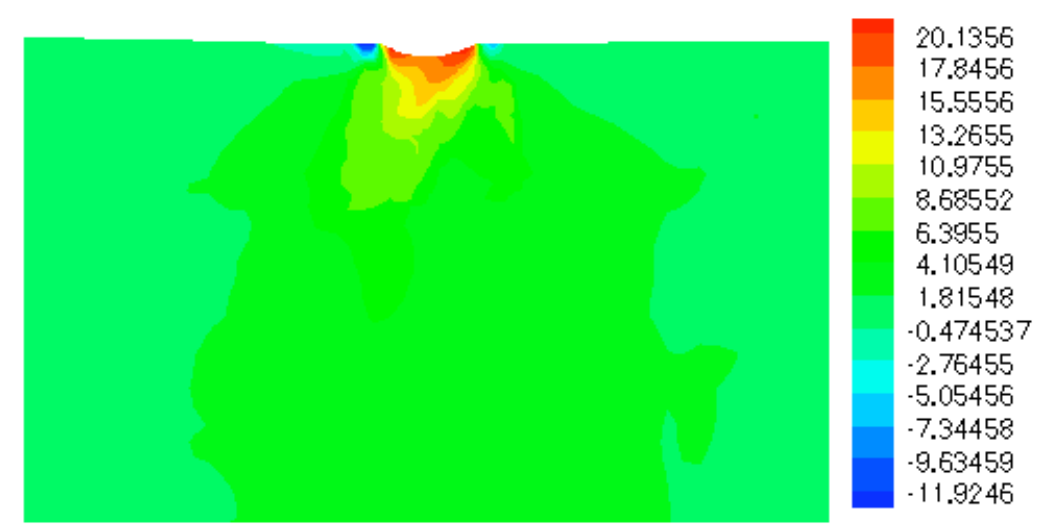

(d)

Figure 8.3. Plastic strain accumulation at final loading step (a), just before unloading step (b), pressure at final loading step normalized by yield stress (c), and pressure just before unloading step normalized by yield stress (d). (Model 1 with $9 \%$ indentation) 


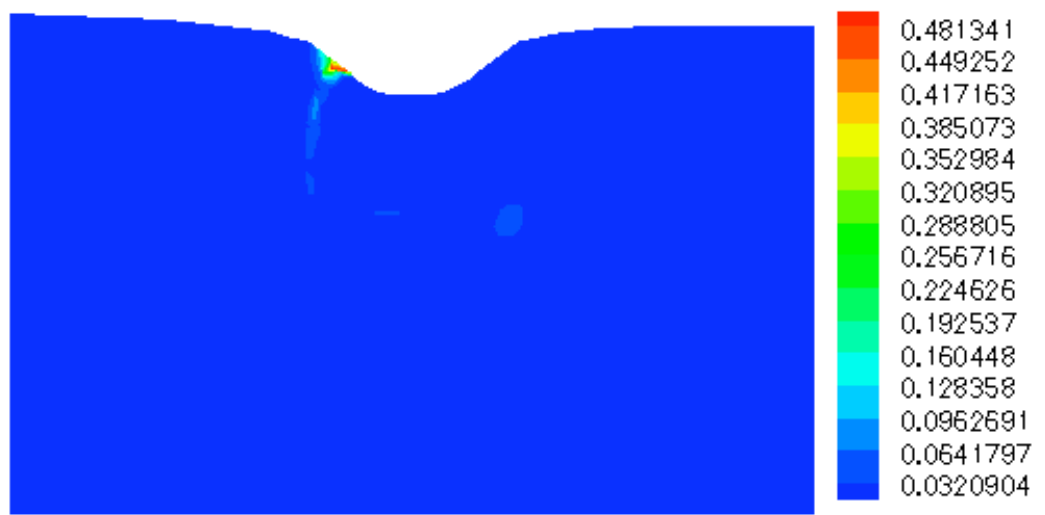

(a)

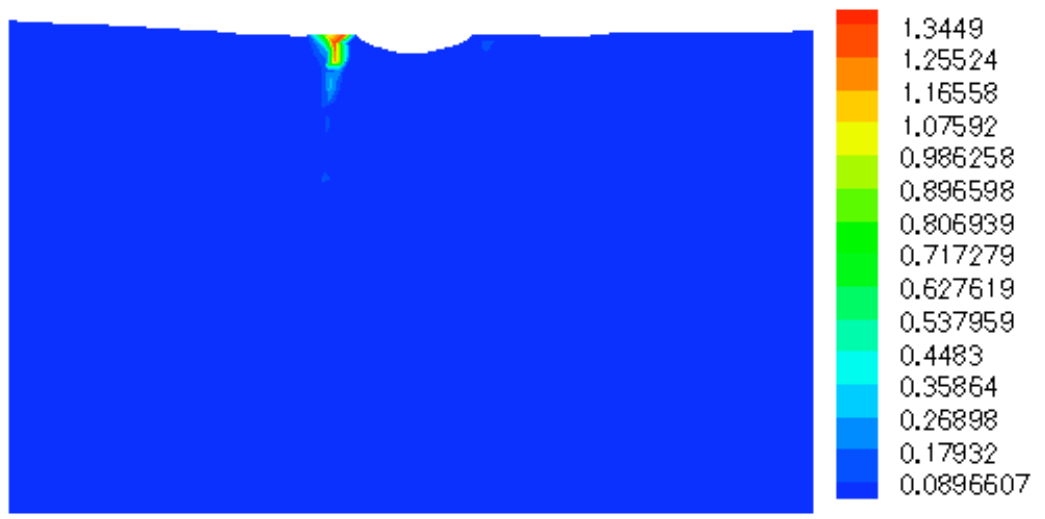

(c)

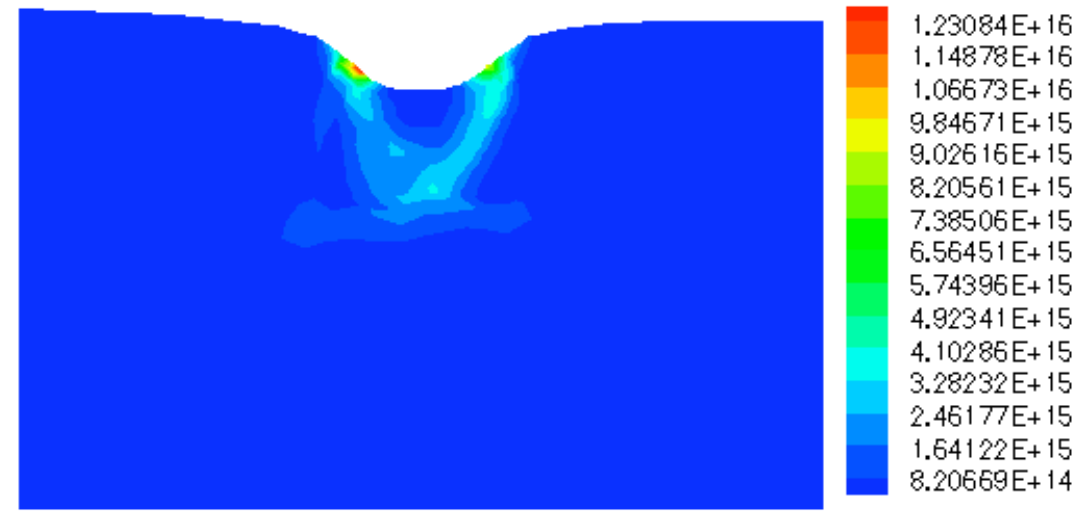

(b)

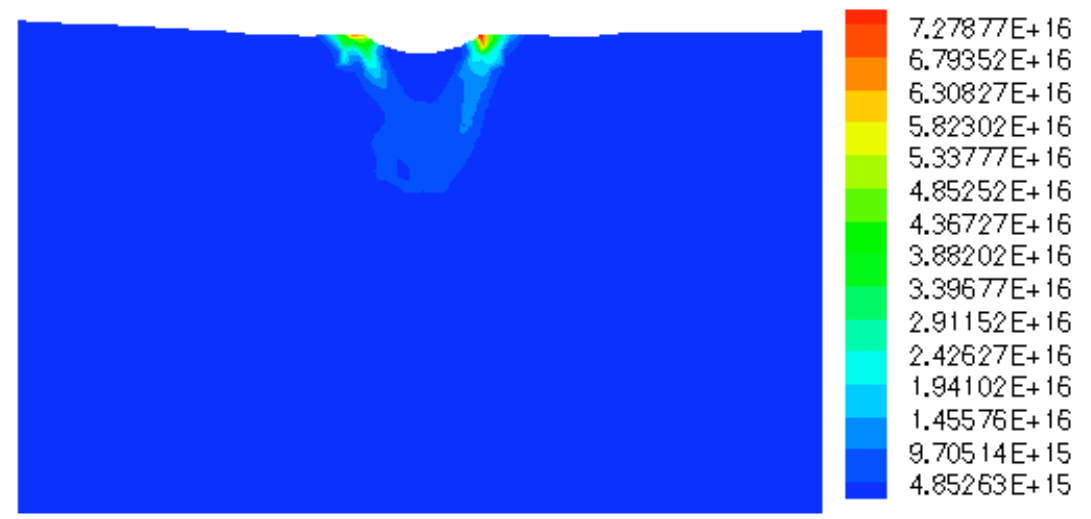

(d)

Figure 8.4. Immobile dislocation-density at final loading step for system 5 normalized by saturation (a), total immobile dislocationdensity at final loading step non-normalized (b), just before unloading step for system 5 normalized by saturation (c), and total immobile dislocation-density just before unloading step non-normalized (d). (Model 1 with $12.8 \%$ indentation) 


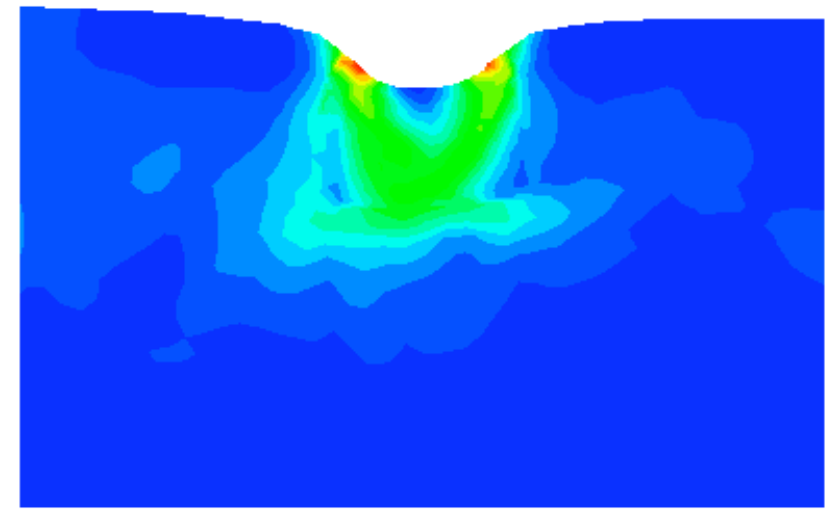

(a)

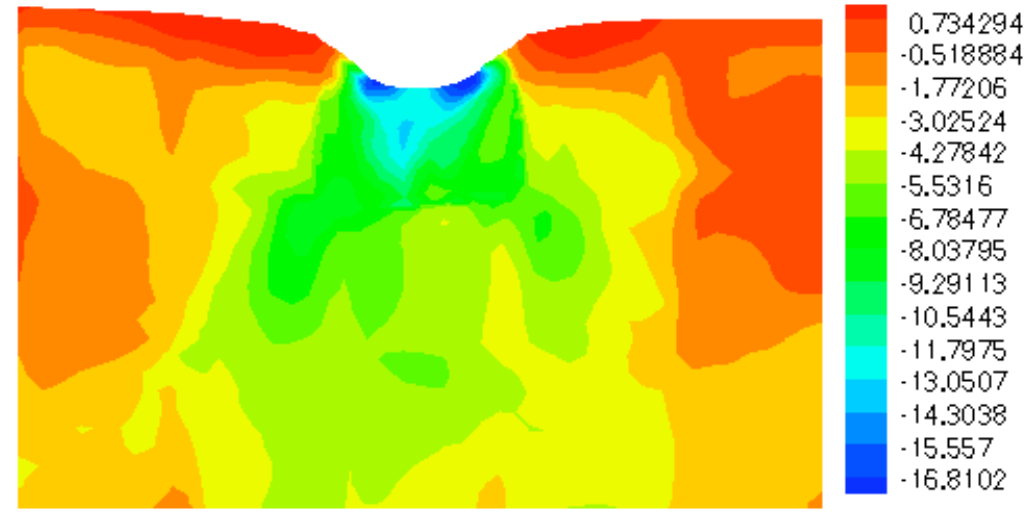

(c)

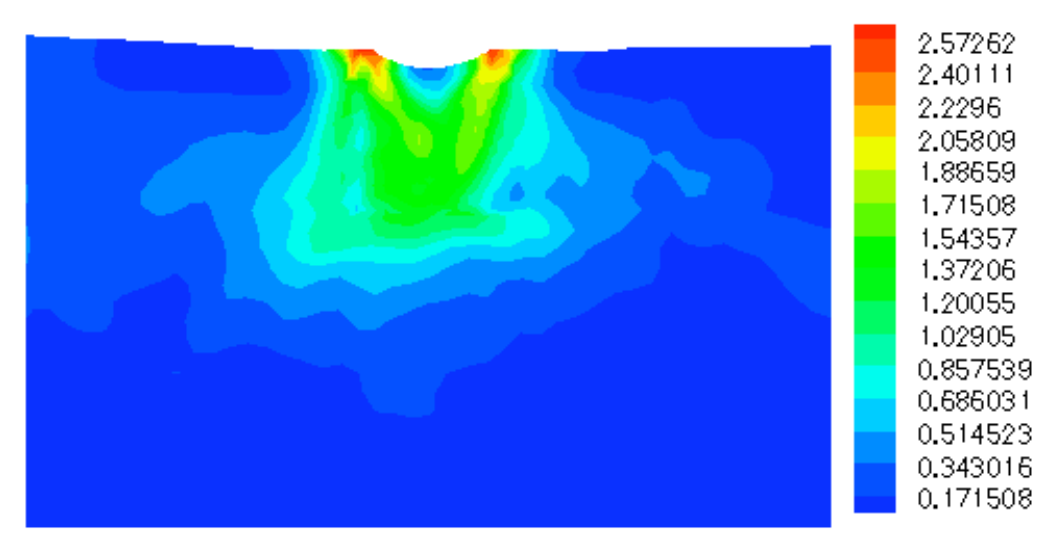

(b)

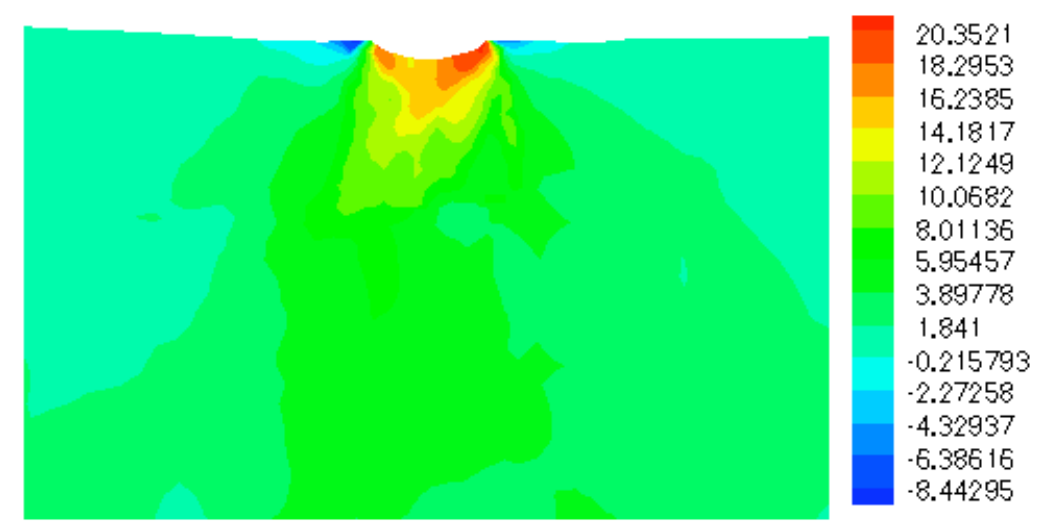

(d)

Figure 8.5. Plastic strain accumulation at final loading step (a), just before unloading step (b), pressure at final loading step normalized by yield stress (c), and pressure just before unloading step normalized by yield stress (d). (Model 1 with $12.8 \%$ indentation) 


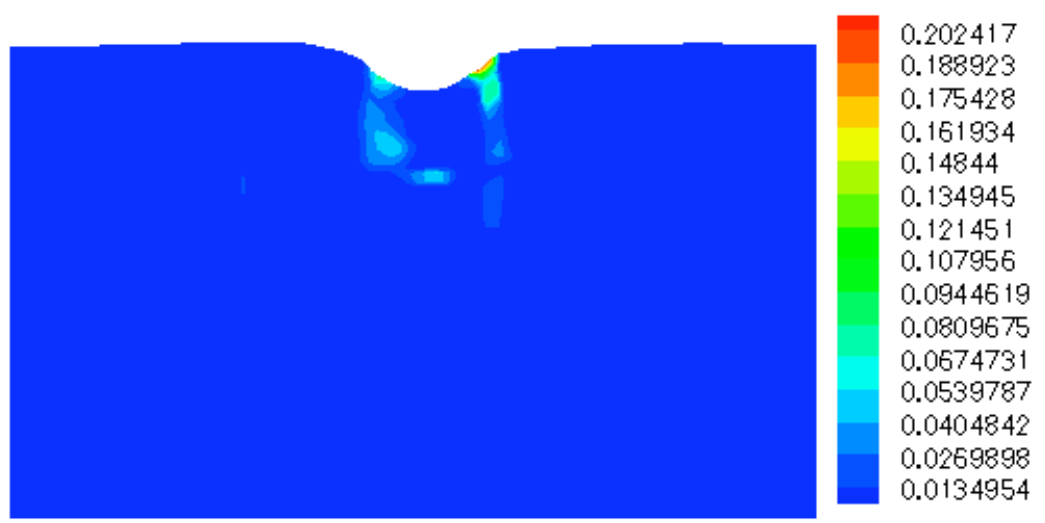

(a)

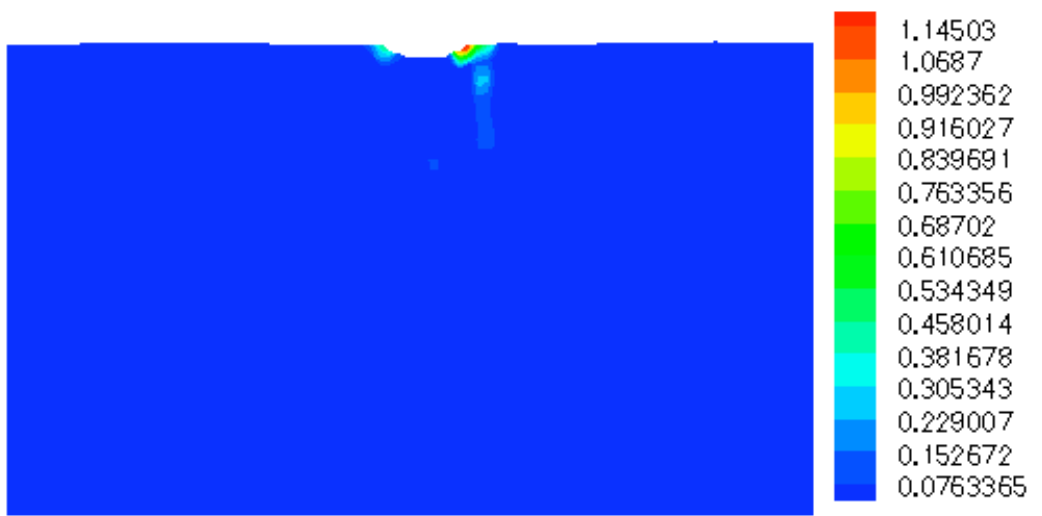

(c)

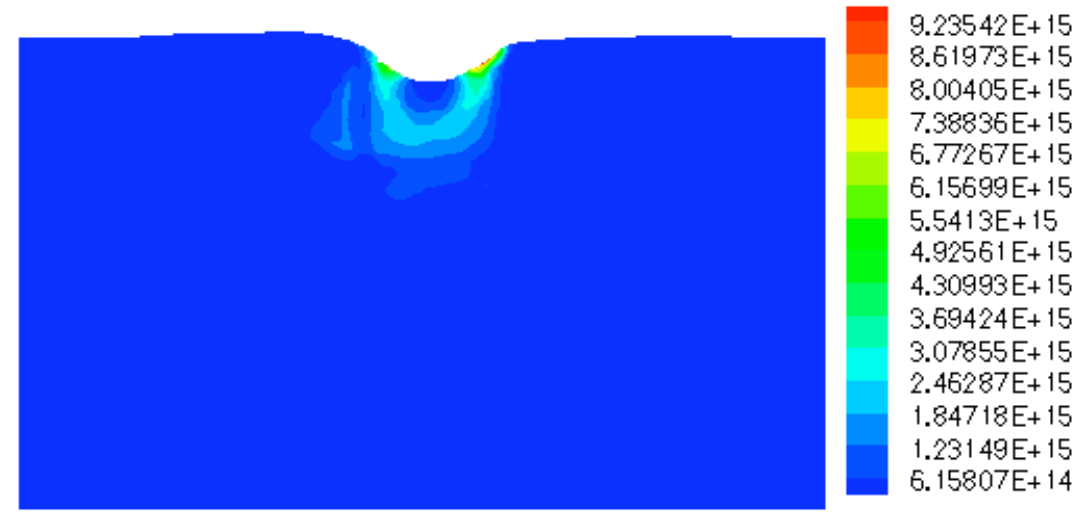

(b)

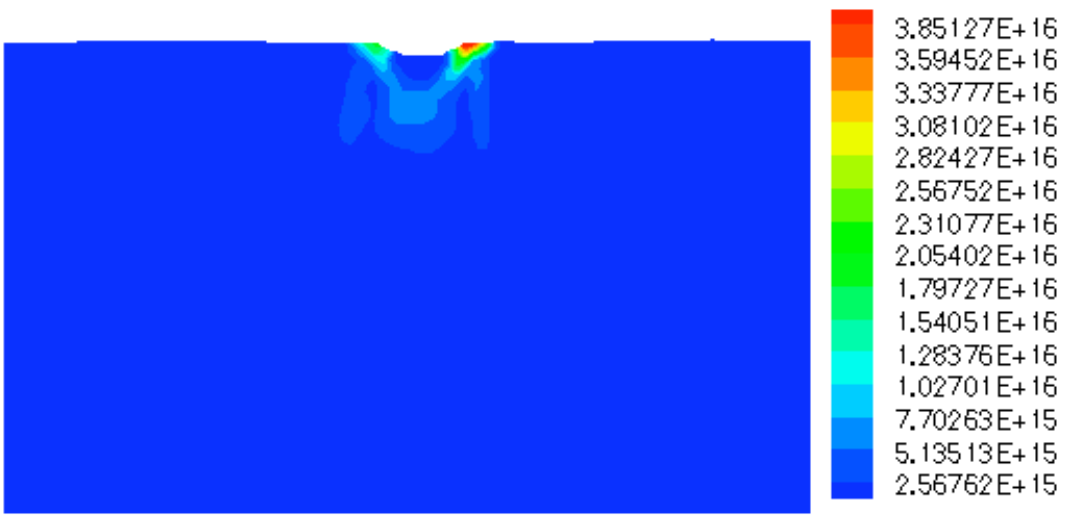

(d)

Figure 8.6. Immobile dislocation-density at final loading step for system 11 normalized by saturation (a), total immobile dislocationdensity at final loading step non-normalized (b), just before unloading step for system 11 normalized by saturation (c), and total immobile dislocation-density just before unloading step non-normalized (d). (Model 2 with 9\% indentation) 


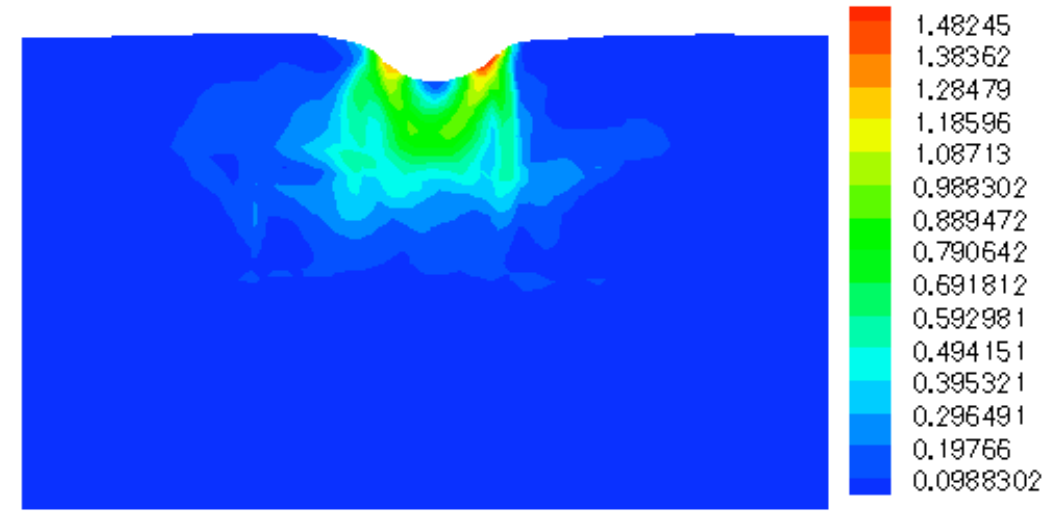

(a)

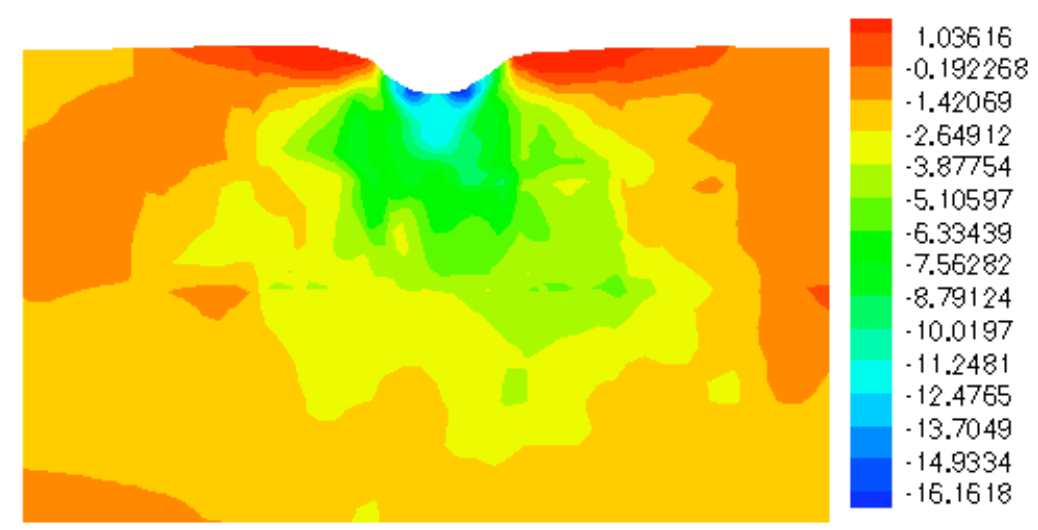

(c)

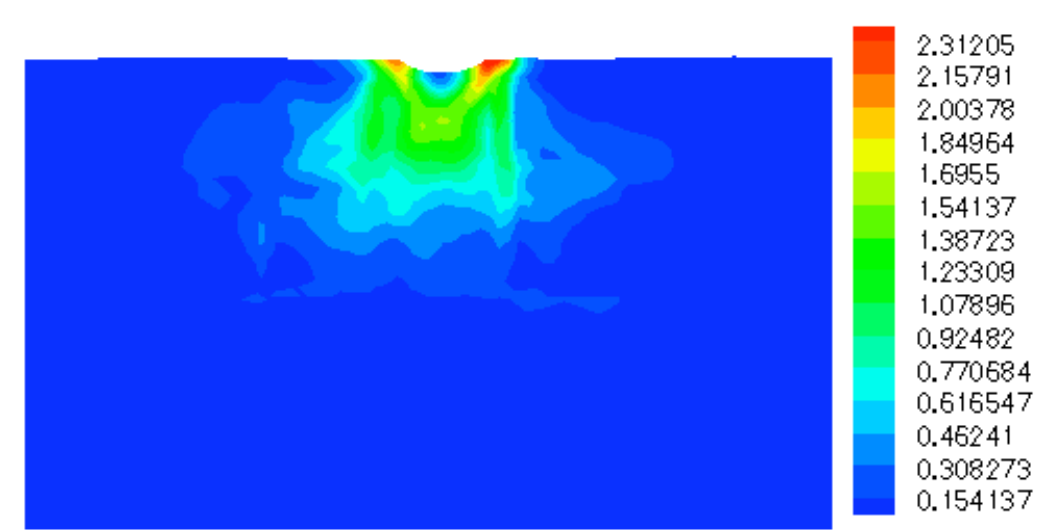

(b)

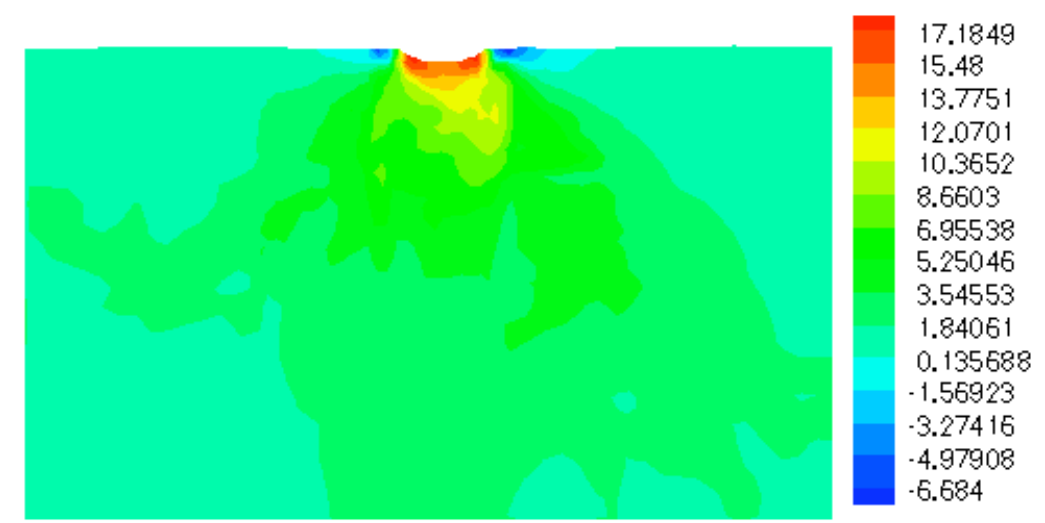

(d)

Figure 8.7. Plastic strain accumulation at final loading step (a), just before unloading step (b), pressure at final loading step normalized by yield stress (c), and pressure just before unloading step normalized by yield stress (d). (Model 2 with $9 \%$ indentation) 


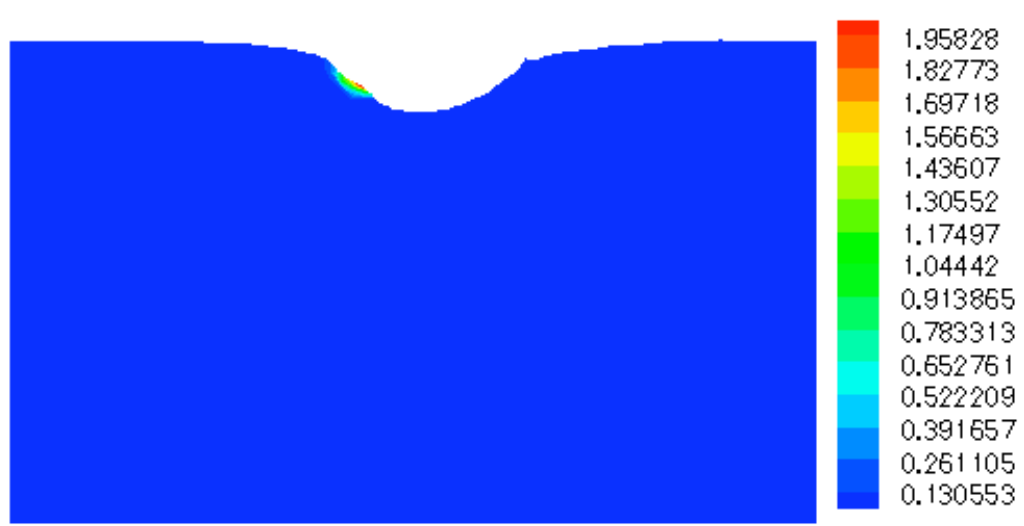

(a)

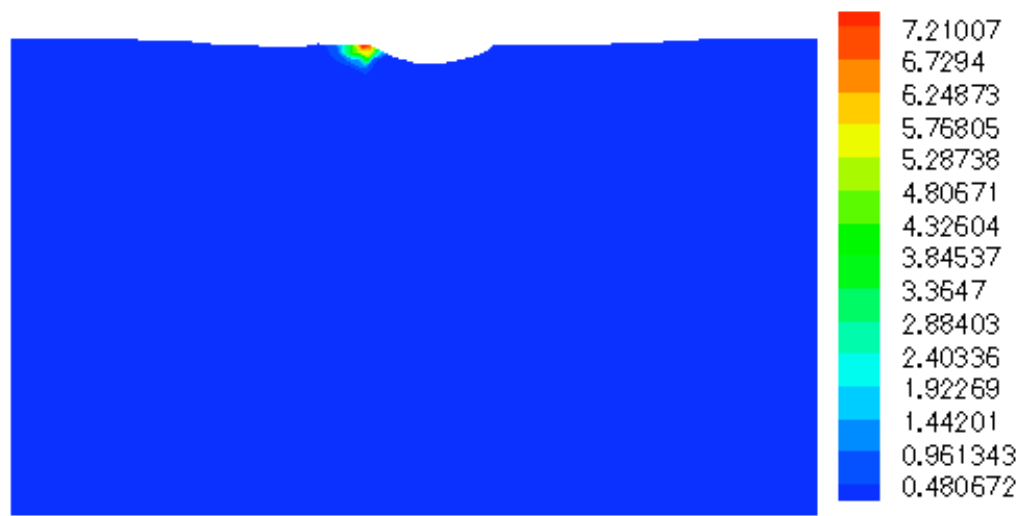

(c)

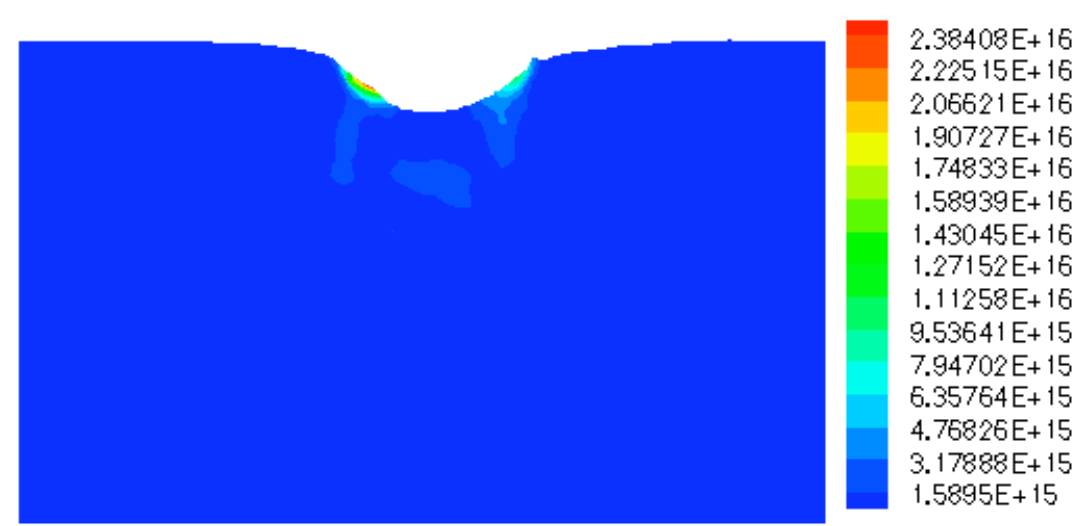

(b)

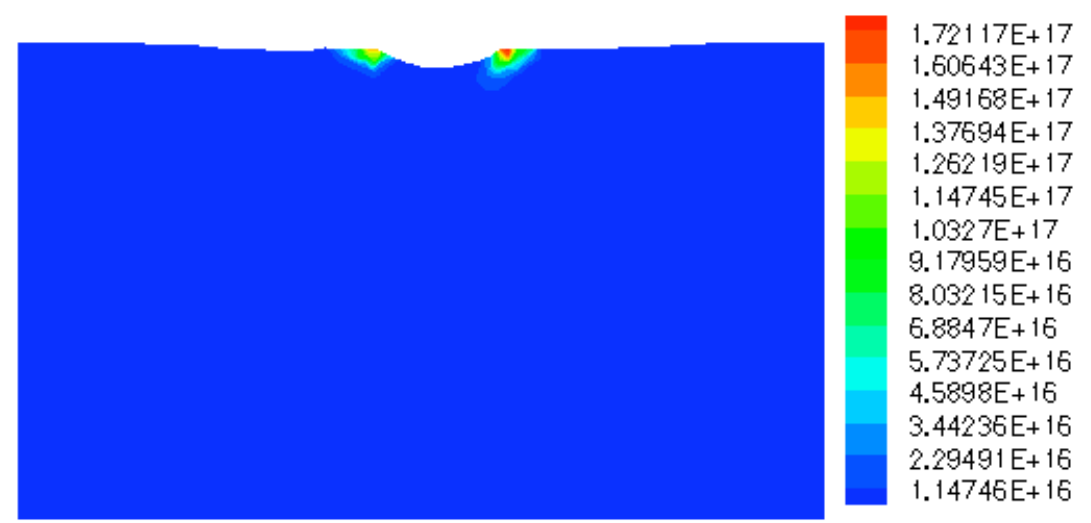

(d)

Figure 8.8. Immobile dislocation-density at final loading step for system 10 normalized by saturation (a), total immobile dislocationdensity at final loading step non-normalized (b), just before unloading step for system 10 normalized by saturation (c), and total immobile dislocation-density just before unloading step non-normalized (d). (Model 2 with 12.8\% indentation) 


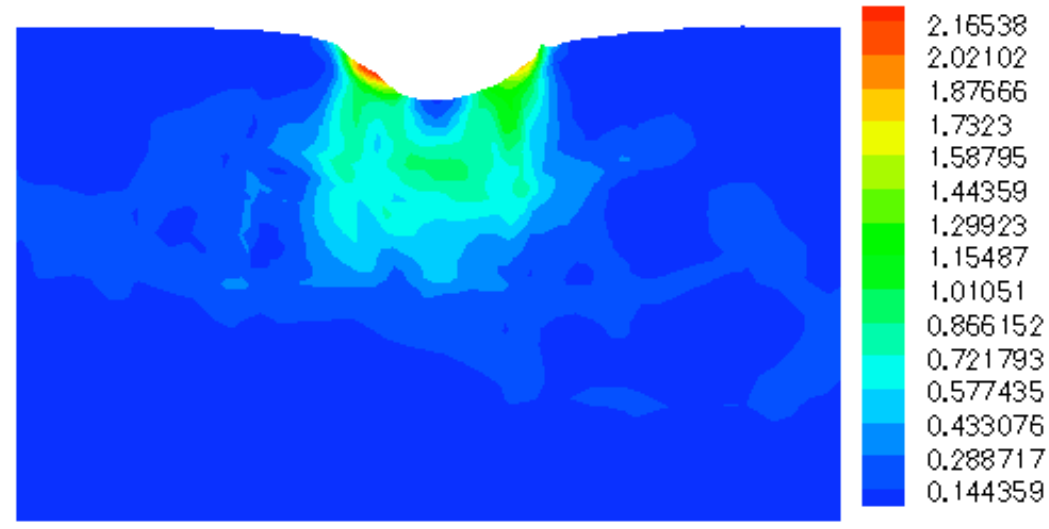

(a)

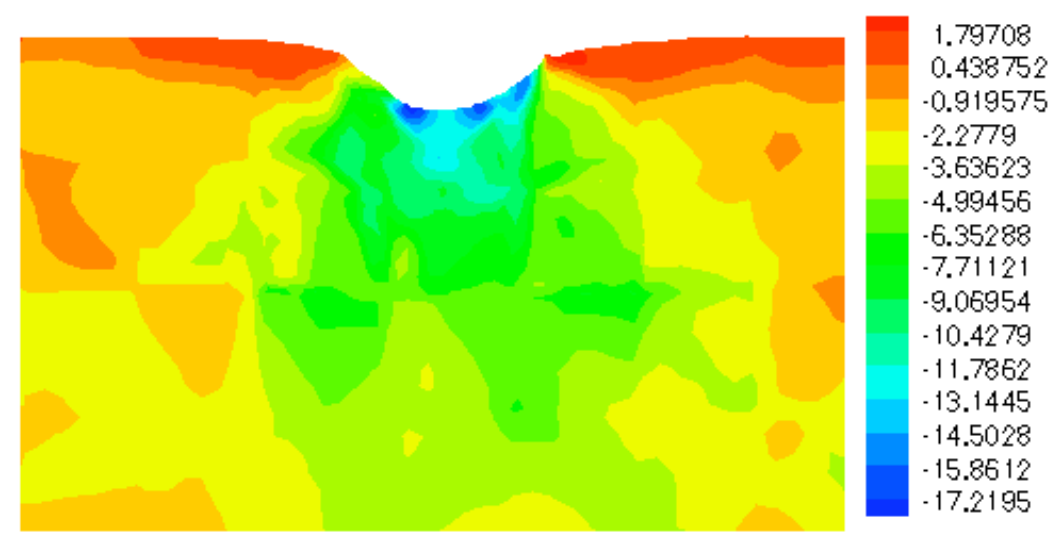

(c)

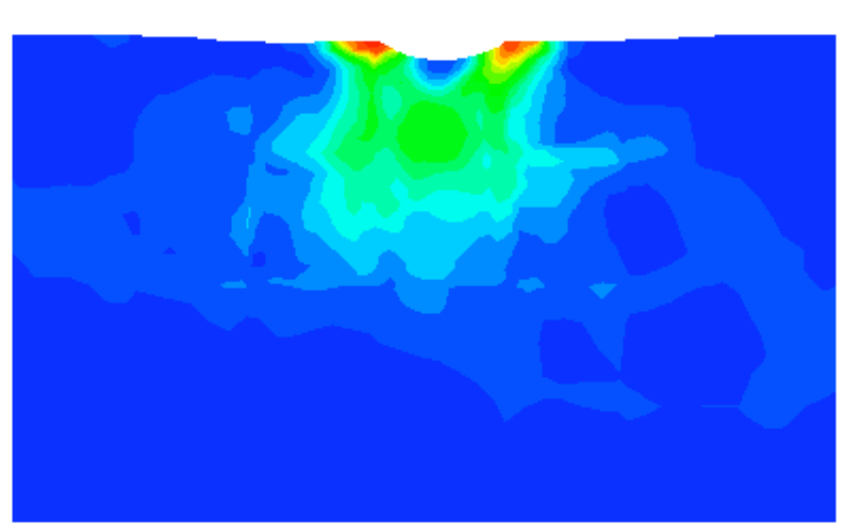

3.03558 2.83321 2.63084 2.42846 2.22609 2.02372 . 1.82135 1.61898 1.4166 1.21423 1.01186 .8098 0.809488 0.607116 0.404744 (b)

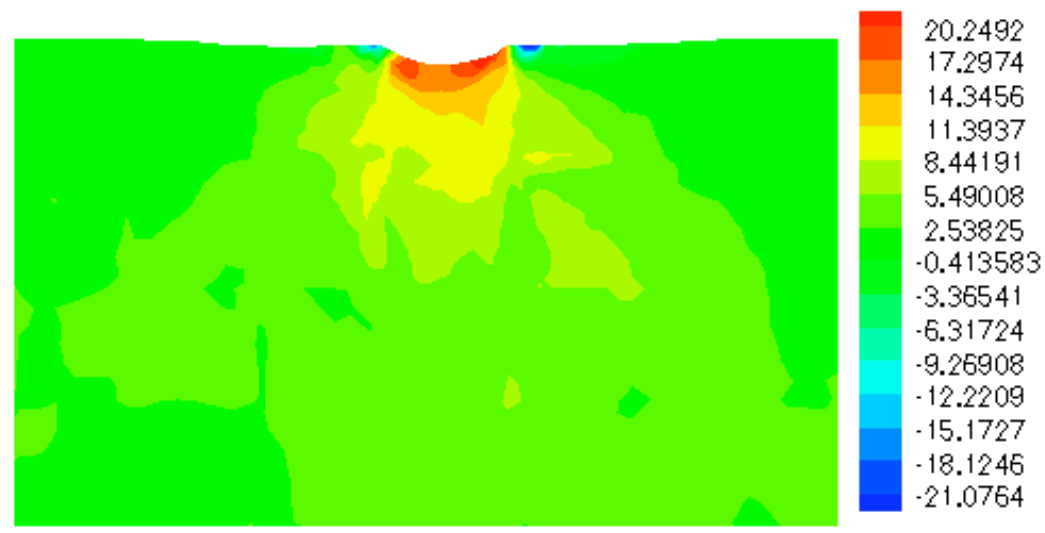

(d)

Figure 8.9. Plastic strain accumulation at final loading step (a), just before unloading step (b), pressure at final loading step normalized by yield stress (c), and pressure just before unloading step normalized by yield stress (d). (Model 2 with 12.8\% indentation) 


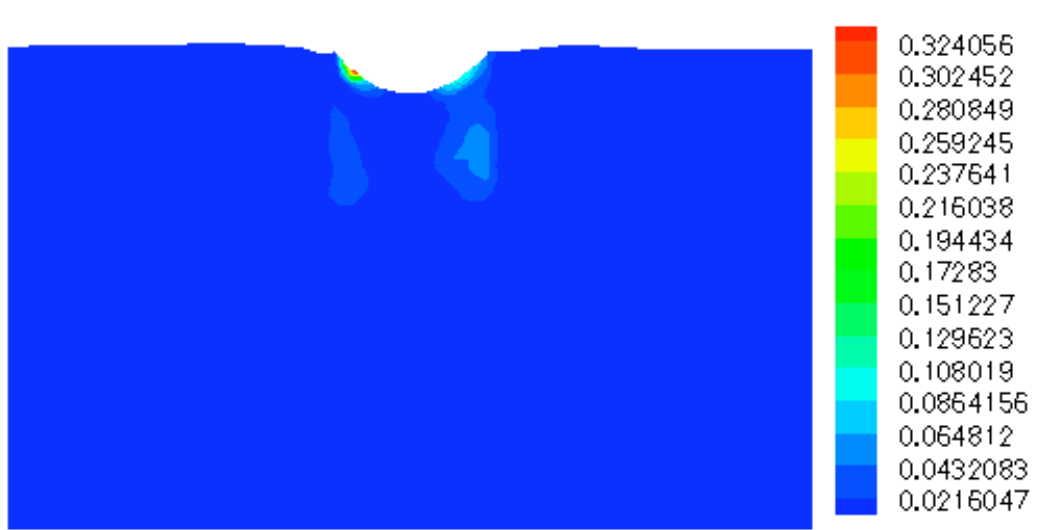

(a)

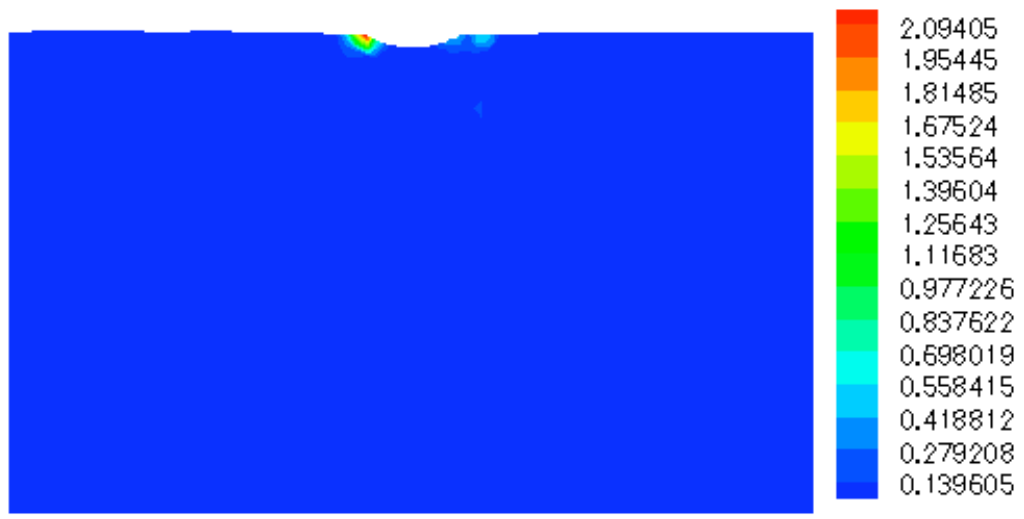

(c)

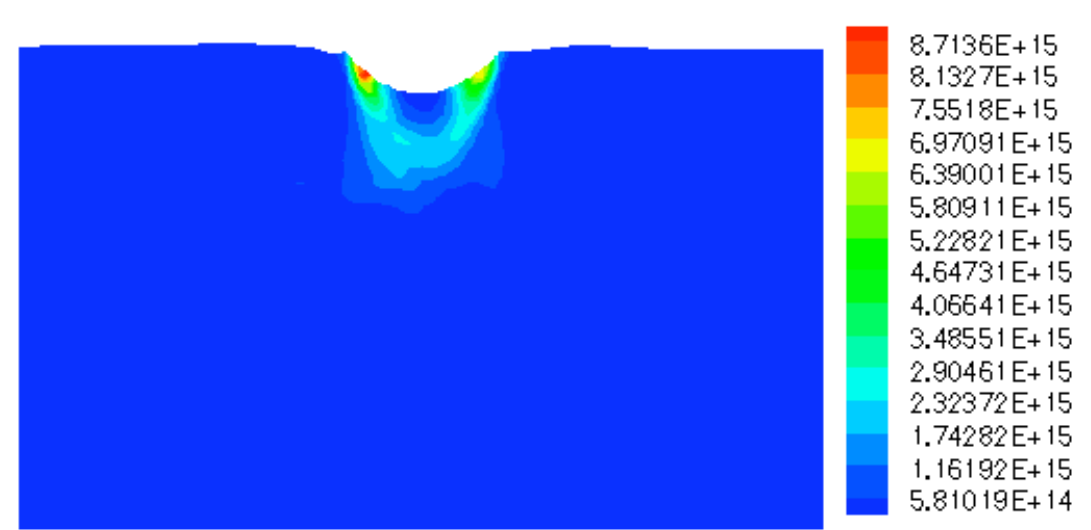

(b)

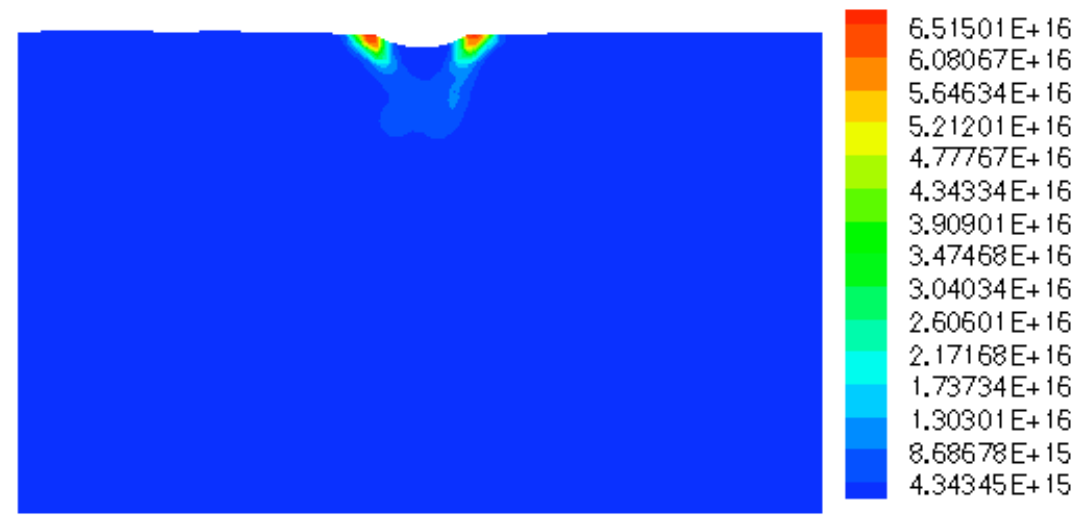

(d)

Figure 8.10. Immobile dislocation-density at final loading step for system 1 normalized by saturation (a), total immobile dislocationdensity at final loading step non-normalized (b), just before unloading step for system 1 normalized by saturation (c), and total immobile dislocation-density just before unloading step non-normalized (d). (Model 3 with 9\% indentation and stronger GBs) 


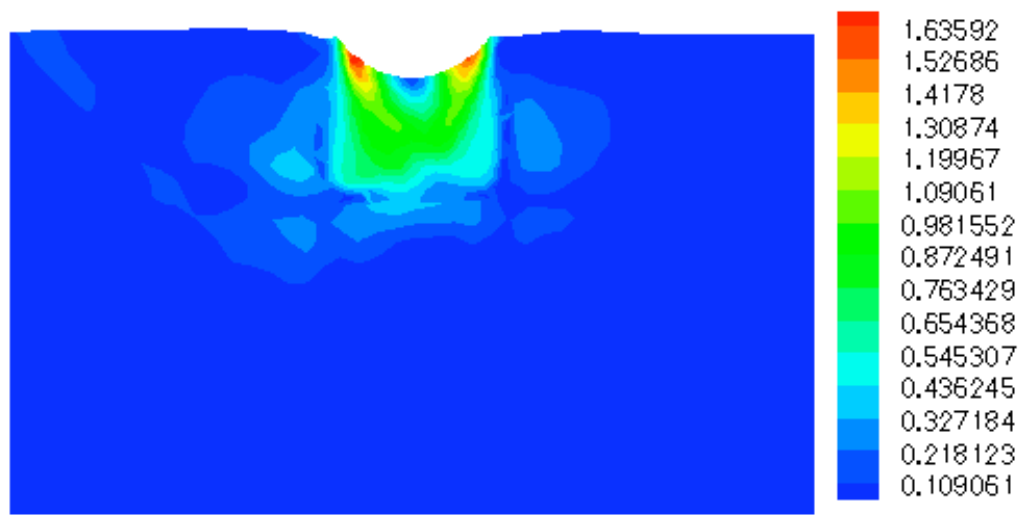

(a)

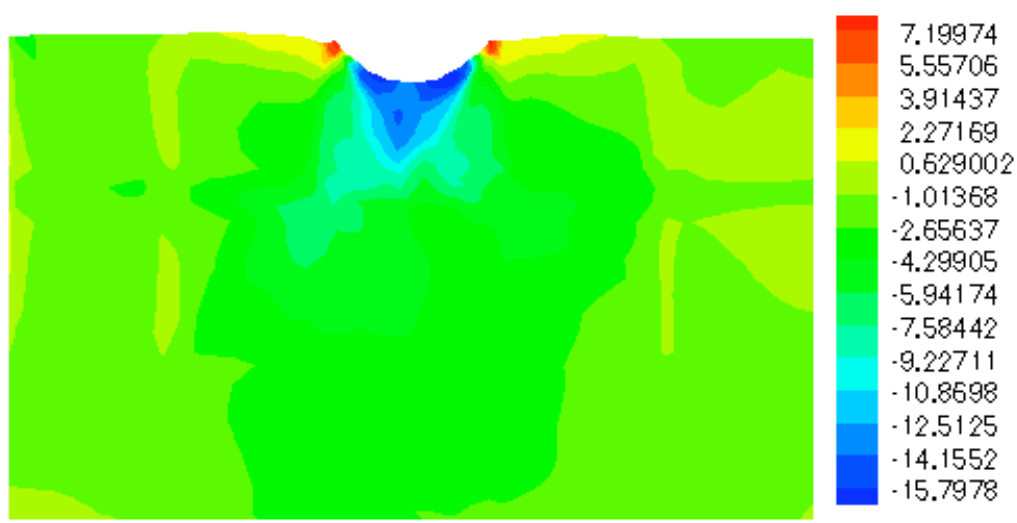

(c)

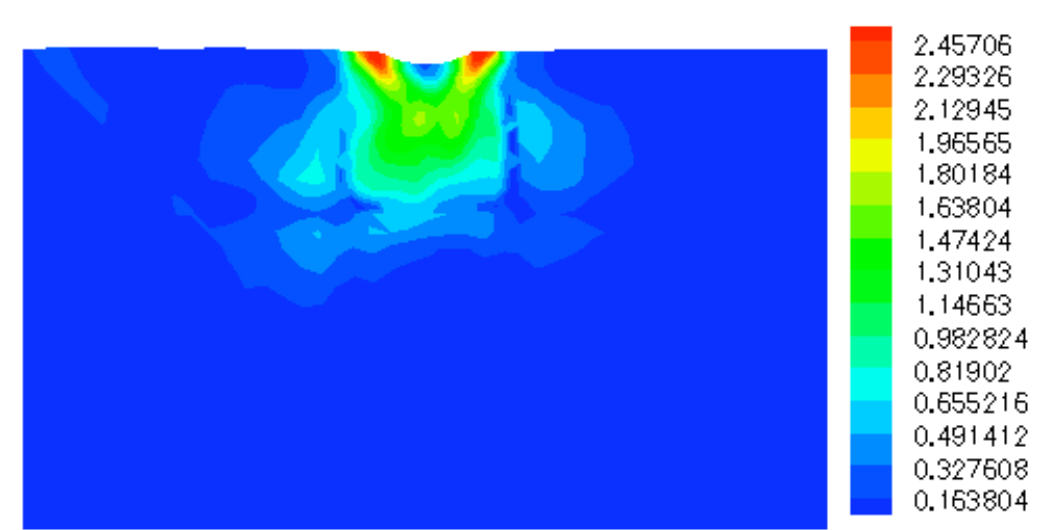

(b)

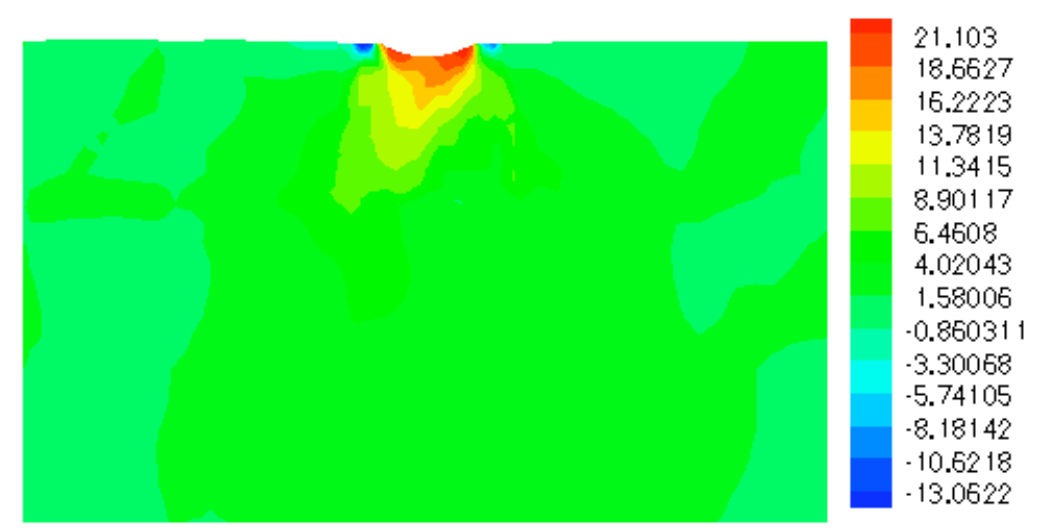

(d)

Figure 8.11. Plastic strain accumulation at final loading step (a), just before unloading step (b), pressure at final loading step normalized by yield stress (c), and pressure just before unloading step normalized by yield stress (d). (Model 3 with $9 \%$ indentation and stronger GBs) 


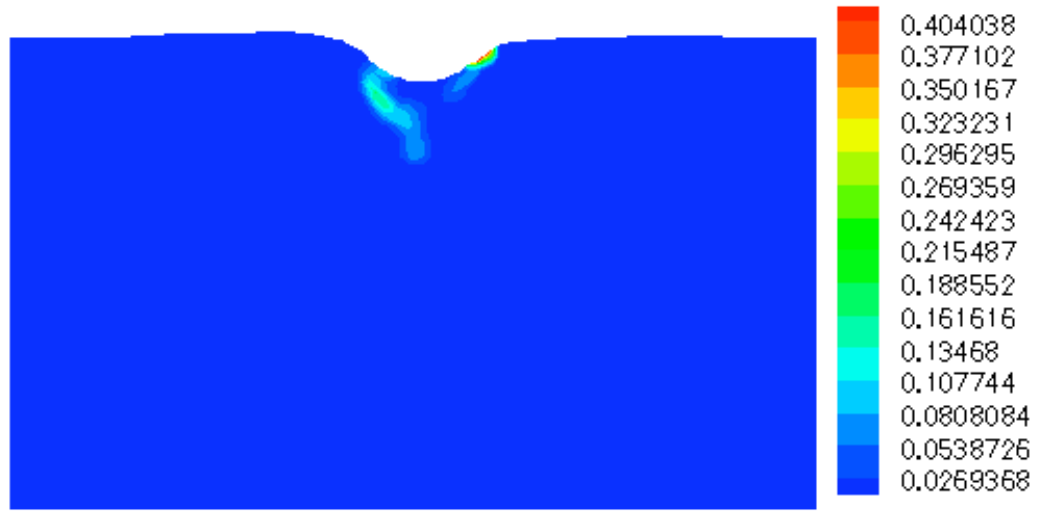

(a)

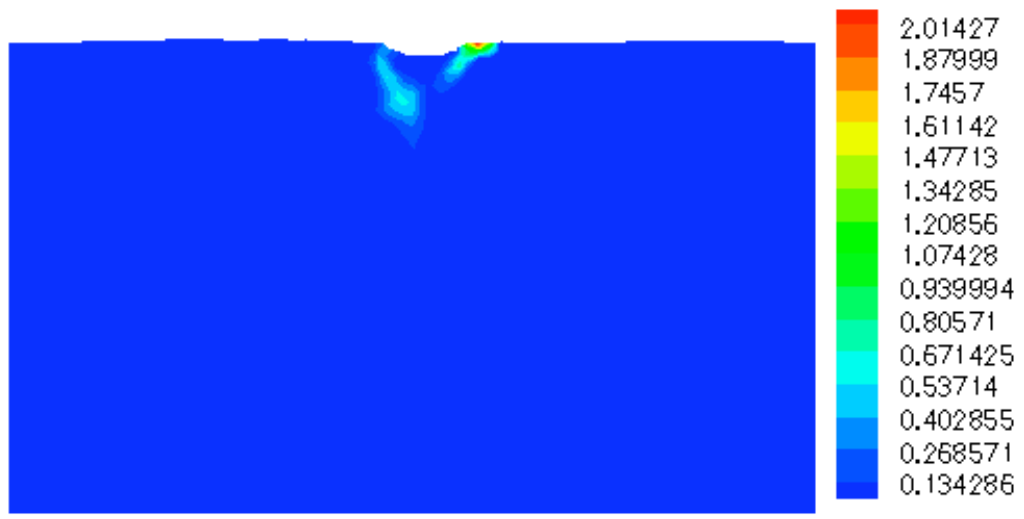

(c)

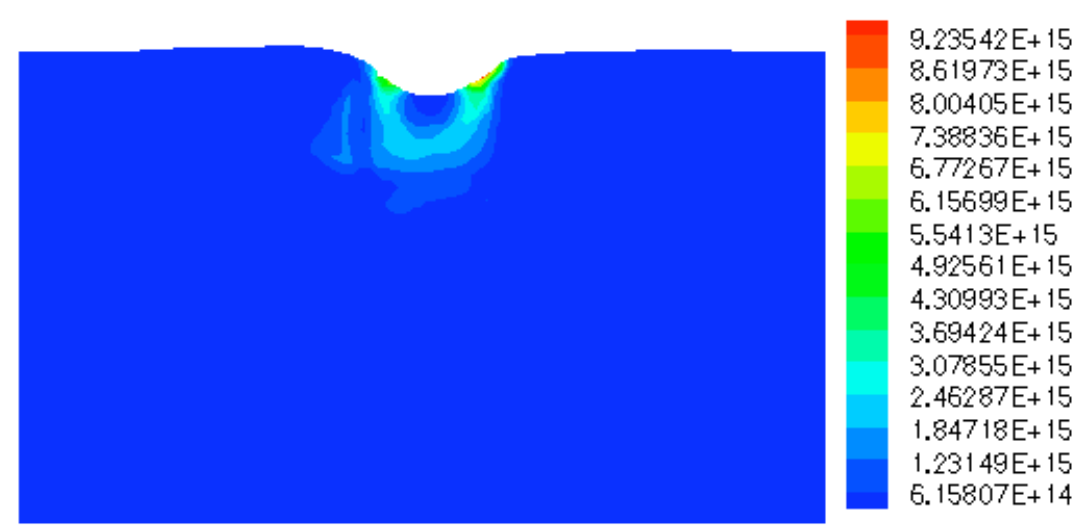

(b)

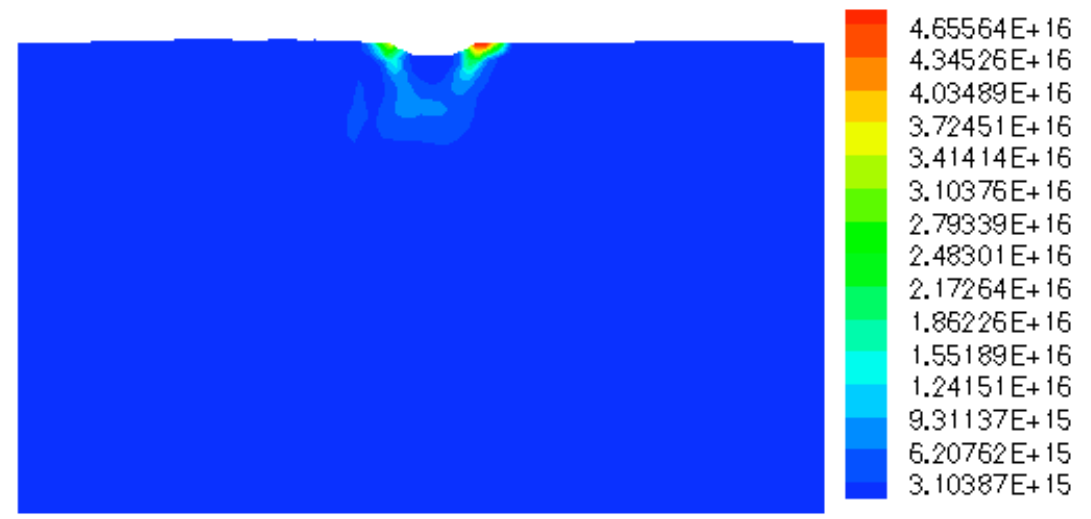

(d)

Figure 8.12. Immobile dislocation-density at final loading step for system 3 normalized by saturation (a), total immobile dislocationdensity at final loading step non-normalized (b), just before unloading step for system 3 normalized by saturation (c), and total immobile dislocation-density just before unloading step non-normalized (d). (Model 4 with 9\% indentation and stronger GBs) 


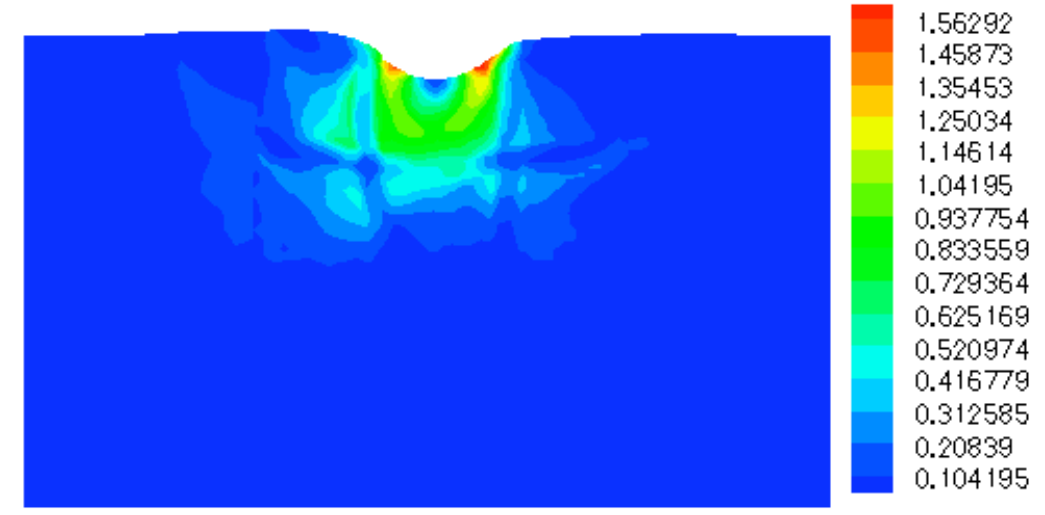

(a)

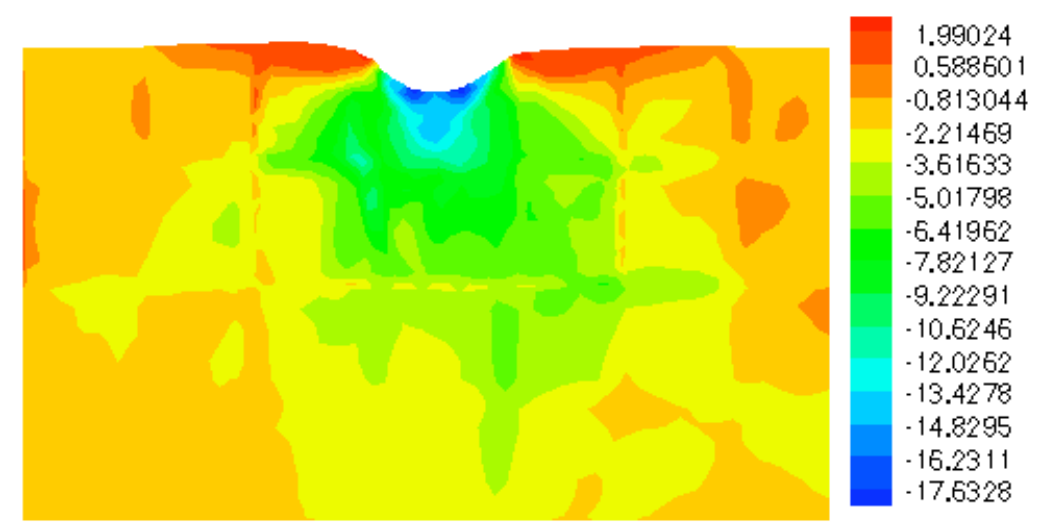

(c)

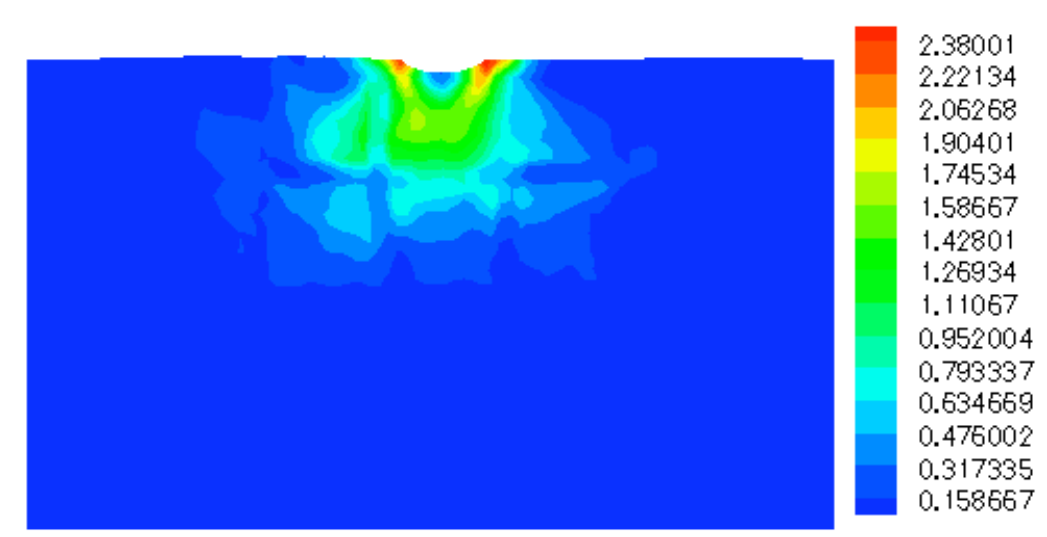

(b)

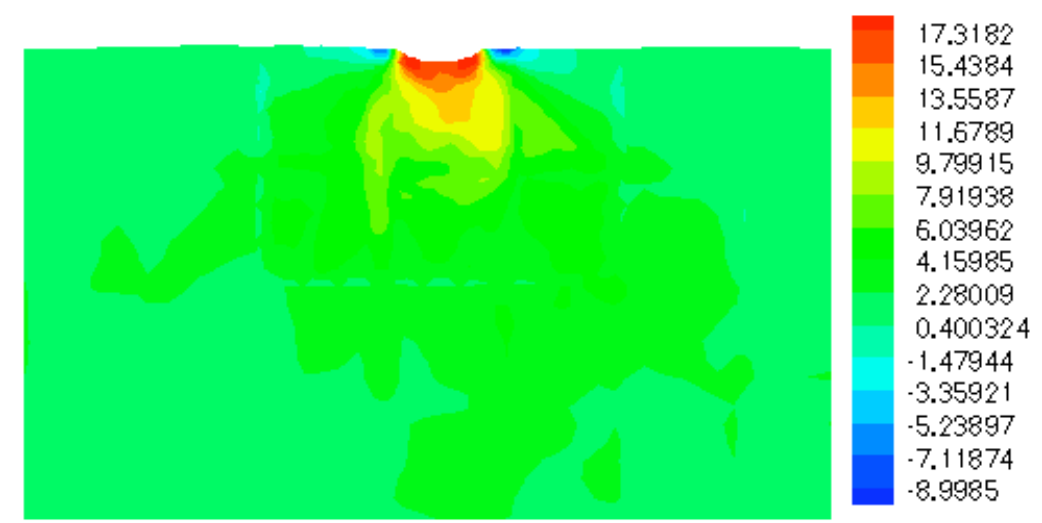

(d)

Figure 8.13. Plastic strain accumulation at final loading step (a), just before unloading step (b), pressure at final loading step normalized by yield stress (c), and pressure just before unloading step normalized by yield stress (d). (Model 4 with $9 \%$ indentation 


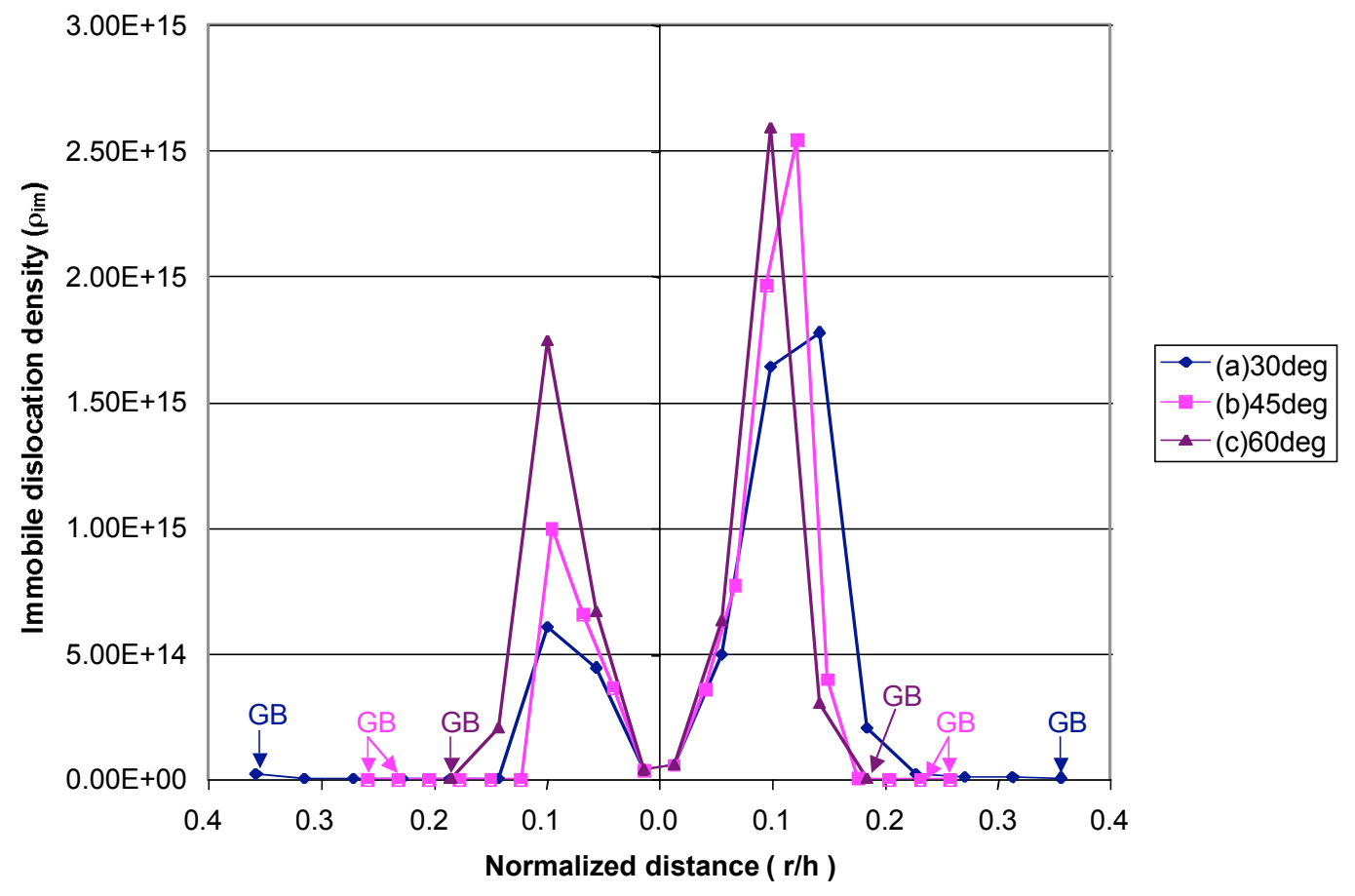

(i)

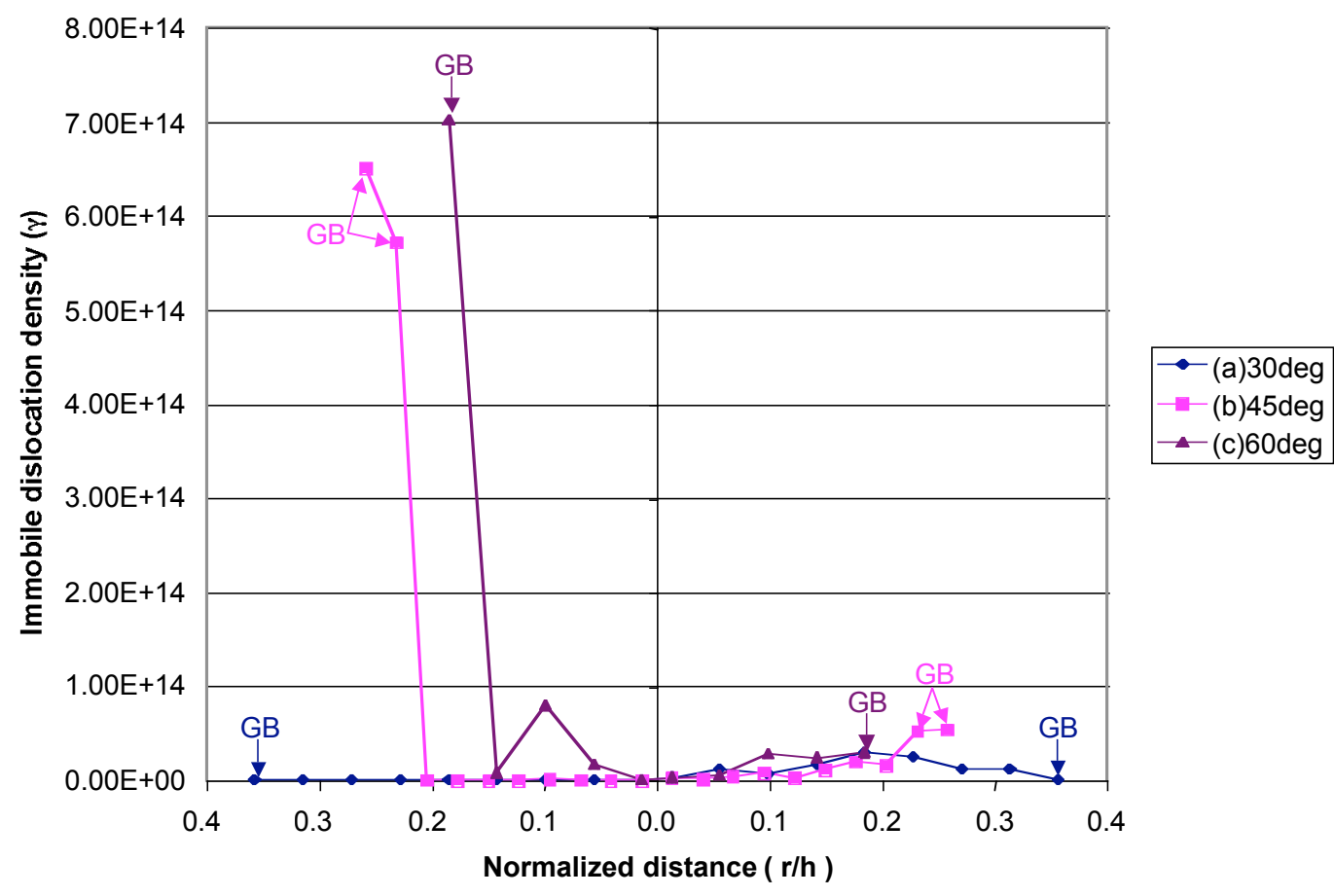

(ii)

Figure 8.14. Immobile dislocation-density distribution along the different angles with respect to loading axis at final loading - 15 grains and high angle GB orientation;

(a) $\pm 30^{\circ}(\mathrm{GB}: \mathrm{r} / \mathrm{h}= \pm 0.3569)$, (b) $\pm 45^{\circ}$ (GB: $\mathrm{r} / \mathrm{h}=0.2312$ and 0.2584$),(\mathrm{c}) \pm 60^{\circ}(\mathrm{GB}: \mathrm{r} / \mathrm{h}=0.185)$ (i) $9 \%$, and (ii) $12.8 \%$ 


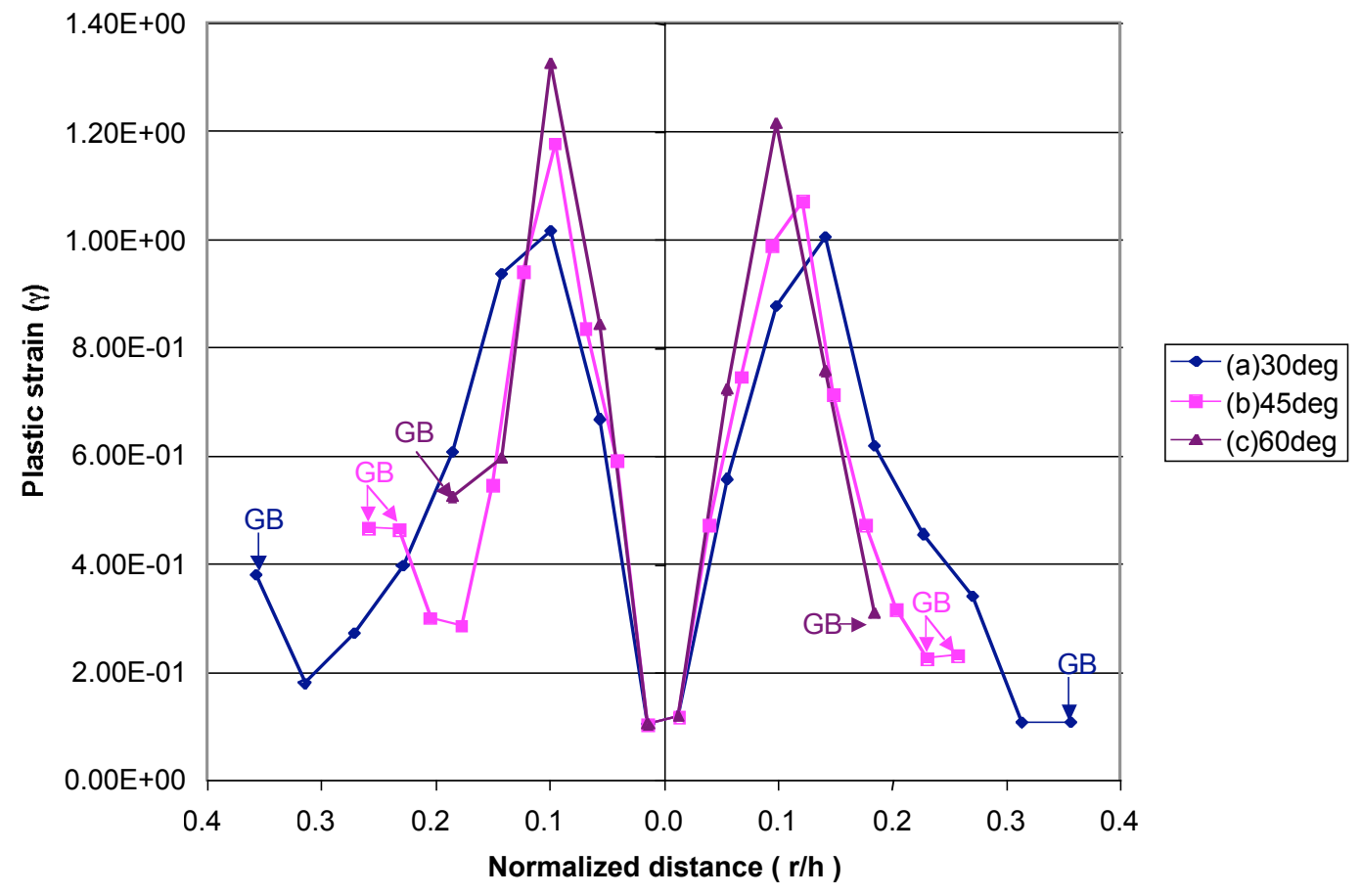

(i)

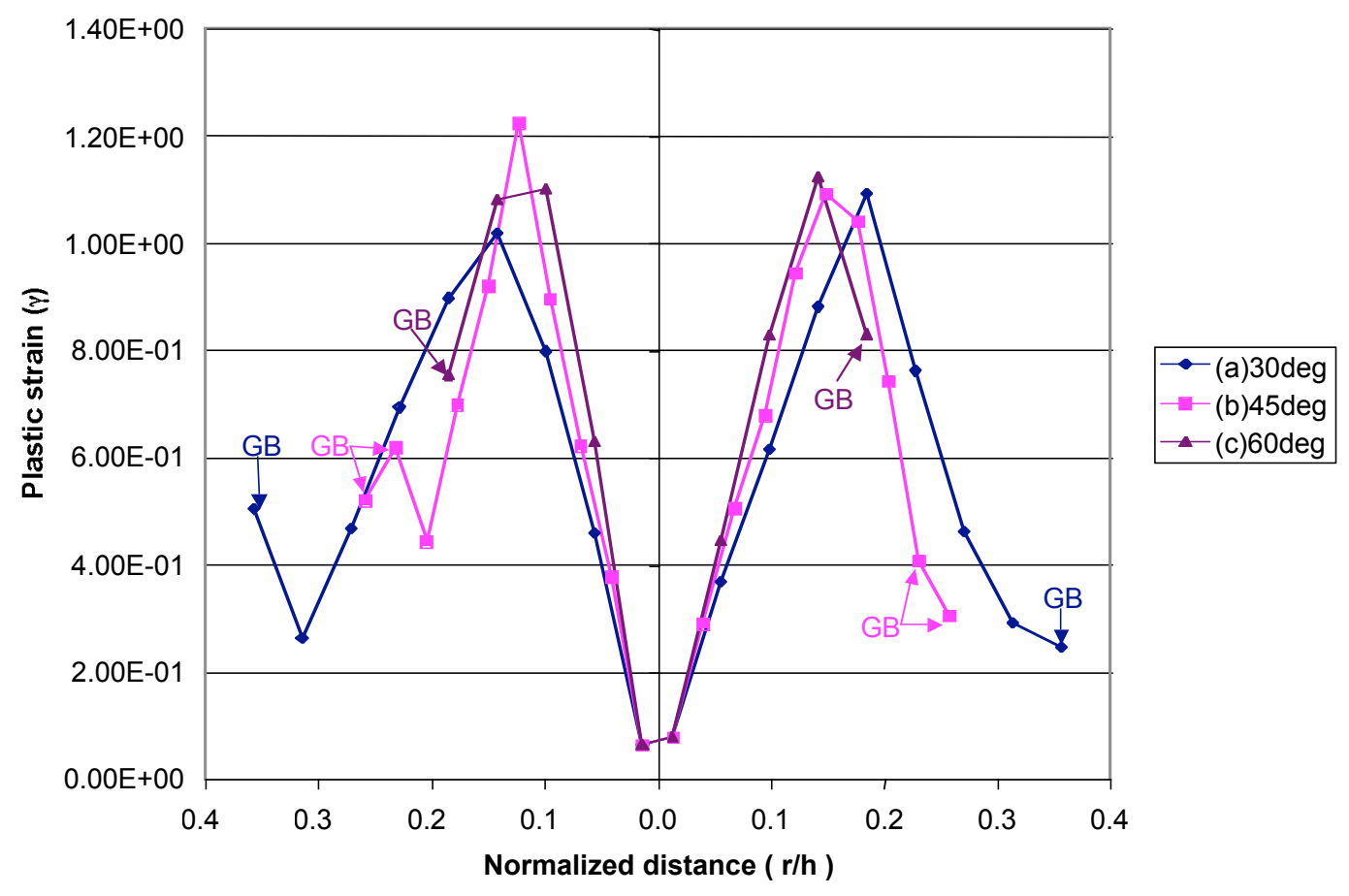

(ii)

Figure 8.15. Plastic strain distribution along the different angles with respect to loading axis at final loading - 15 grains and high angle GB orientation;

(a) $\pm 30^{\circ}$ (GB: $\left.\mathrm{r} / \mathrm{h}=0.3569\right)$, (b) $\pm 45^{\circ}$ (GB: $\mathrm{r} / \mathrm{h}=0.2312$ and 0.2584$)$, (c) $\pm 60^{\circ}$ (GB: $\mathrm{r} / \mathrm{h}=0.185$ )

(i) $9 \%$, and (ii) $12.8 \%$ 


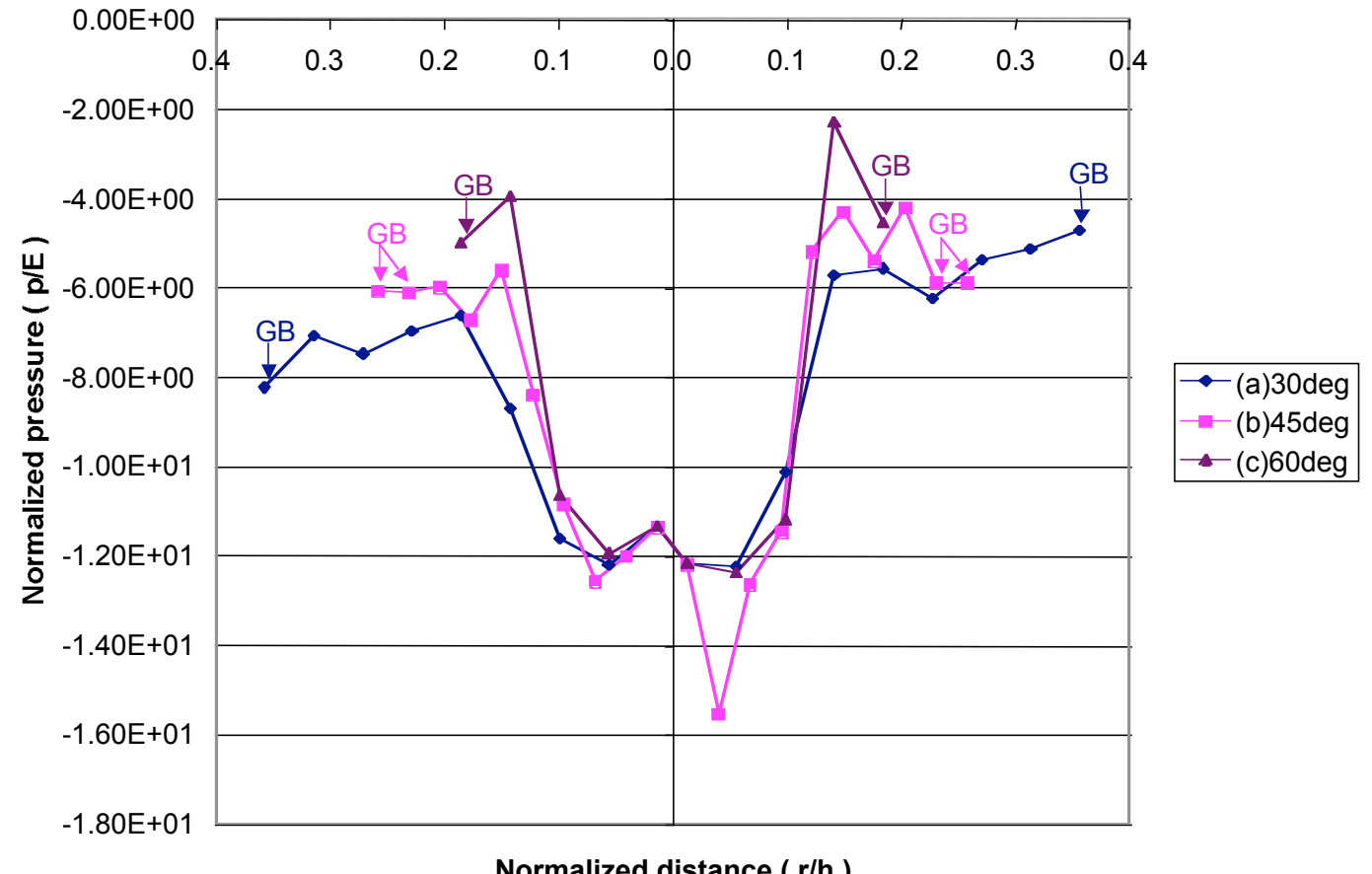

(i)

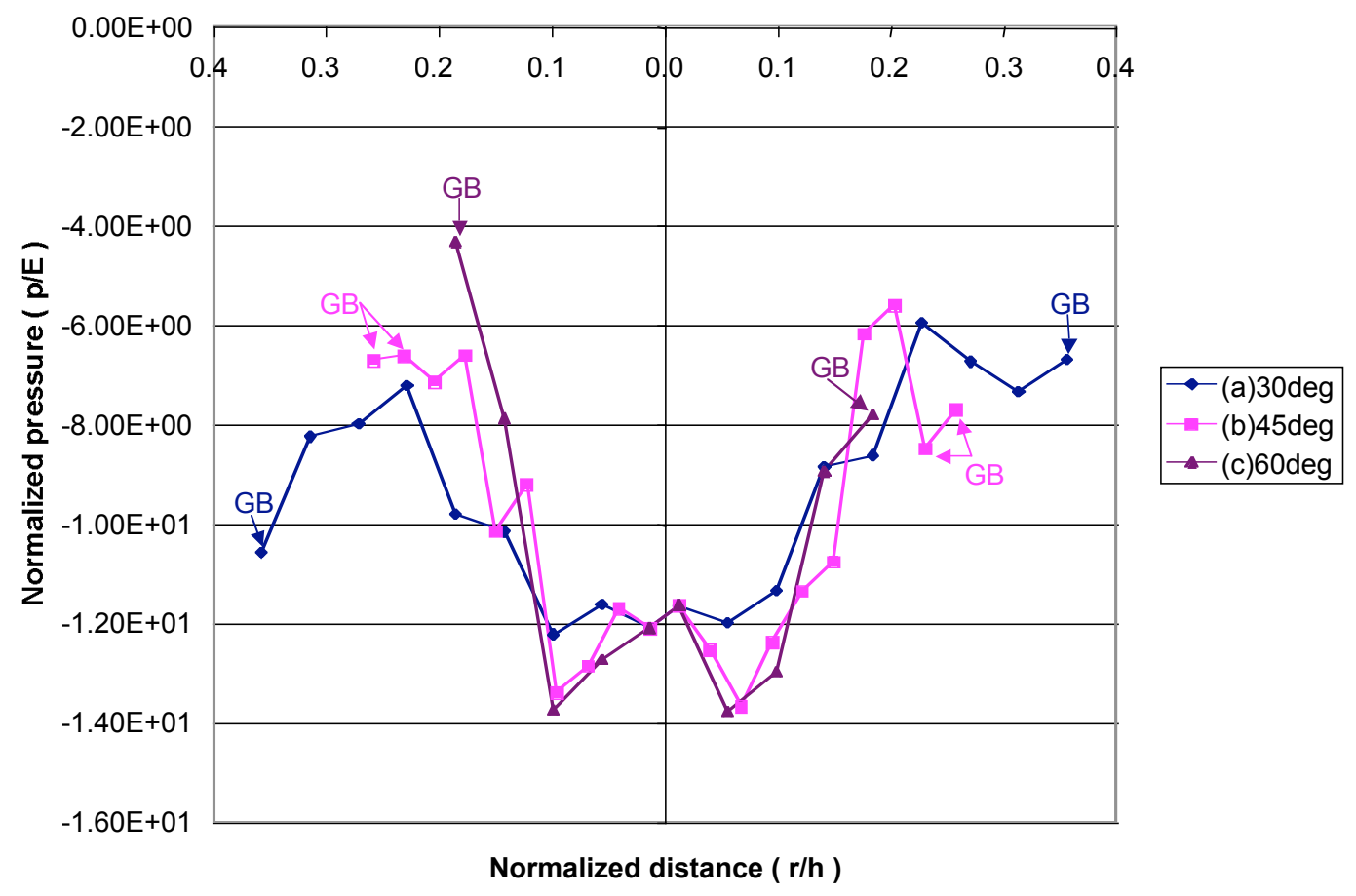

(ii)

Figure 8.16. Normalized pressure distribution along the different angles with respect to loading axis at final loading -15 grains and high angle GB orientation;

(a) $\pm 30^{\circ}$ (GB: $\left.\mathrm{r} / \mathrm{h}=0.3569\right)$, (b) $\pm 45^{\circ}$ (GB: $\mathrm{r} / \mathrm{h}=0.2312$ and 0.2584$)$, (c) $\pm 60^{\circ}$ (GB: $\left.\mathrm{r} / \mathrm{h}=0.185\right)$ (i) $9 \%$, and (ii) $12.8 \%$ 

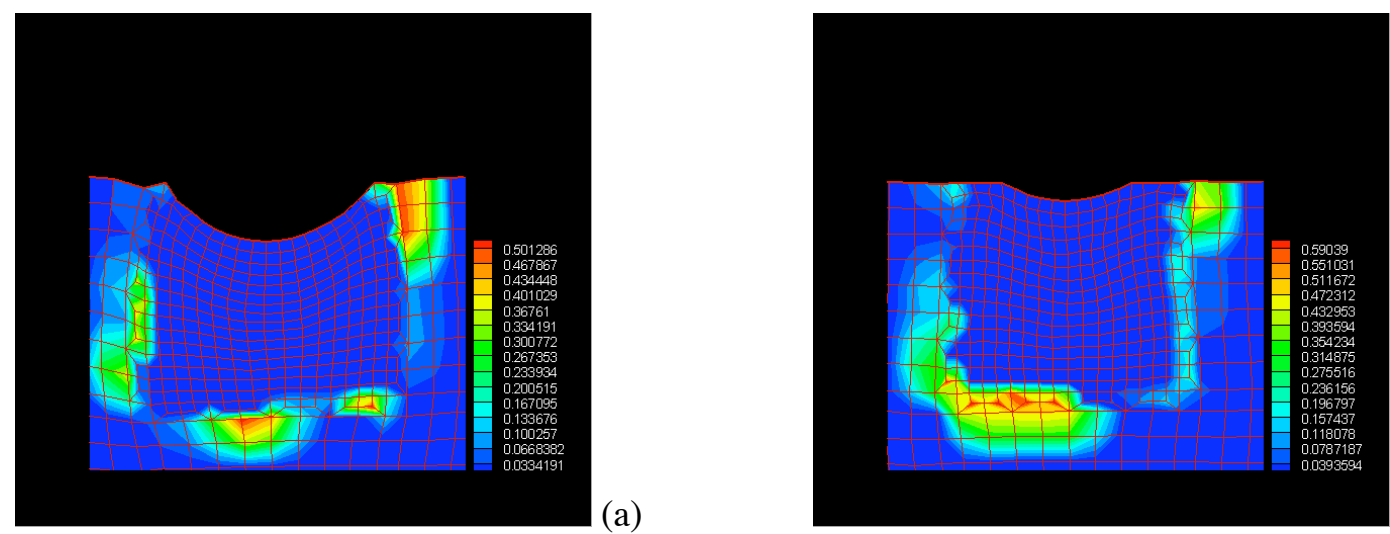

(a)

(b)

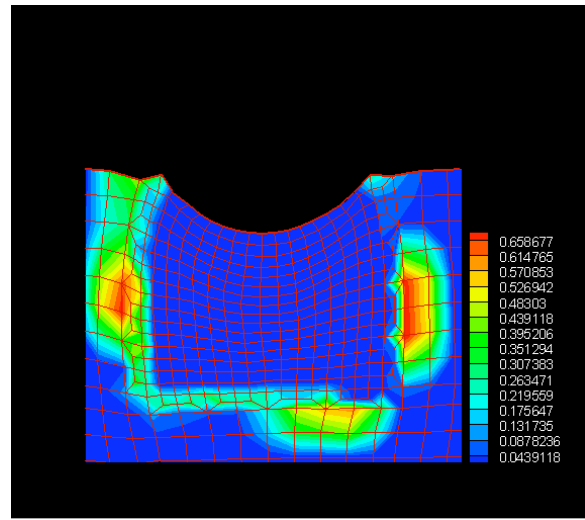

(c)
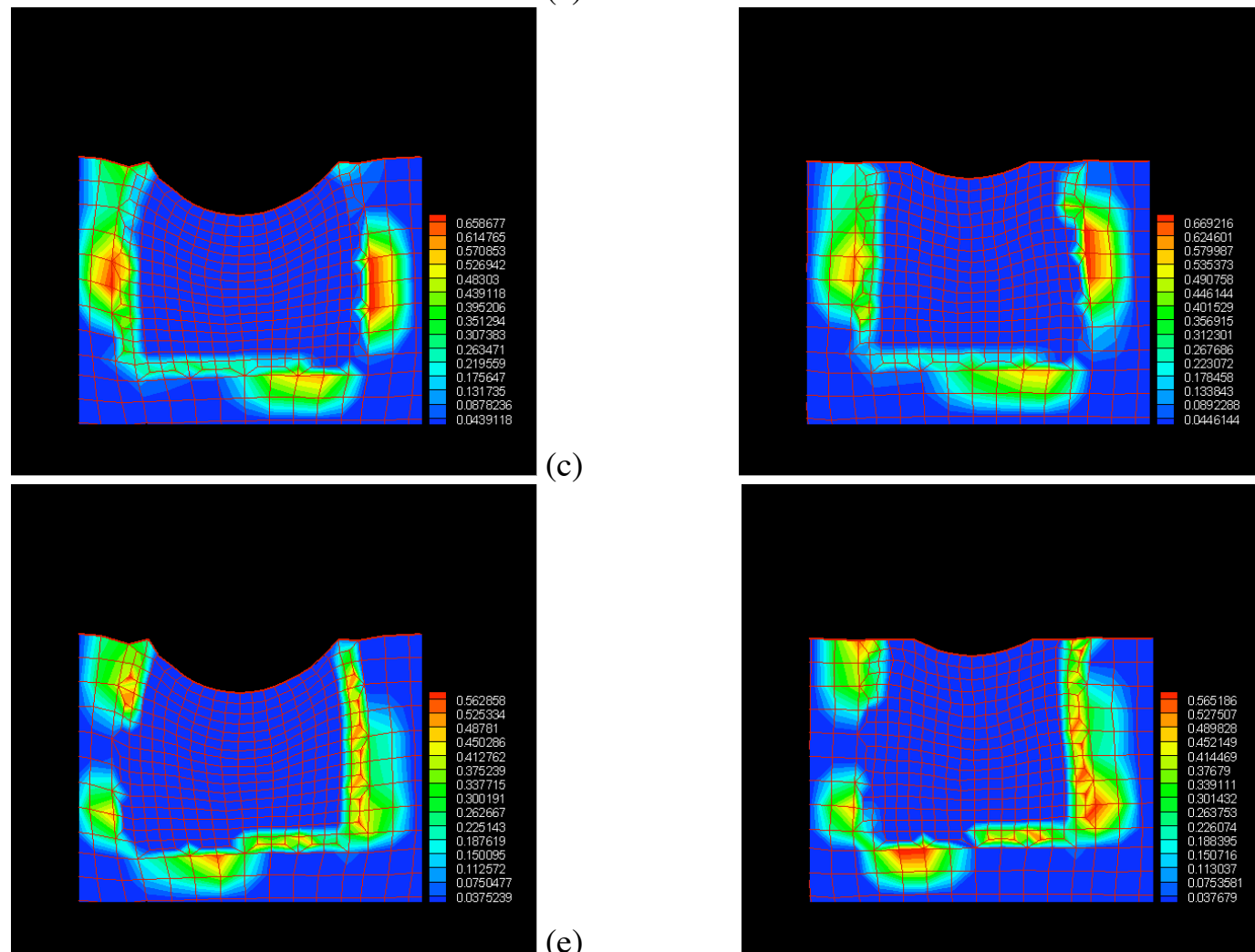

(e)
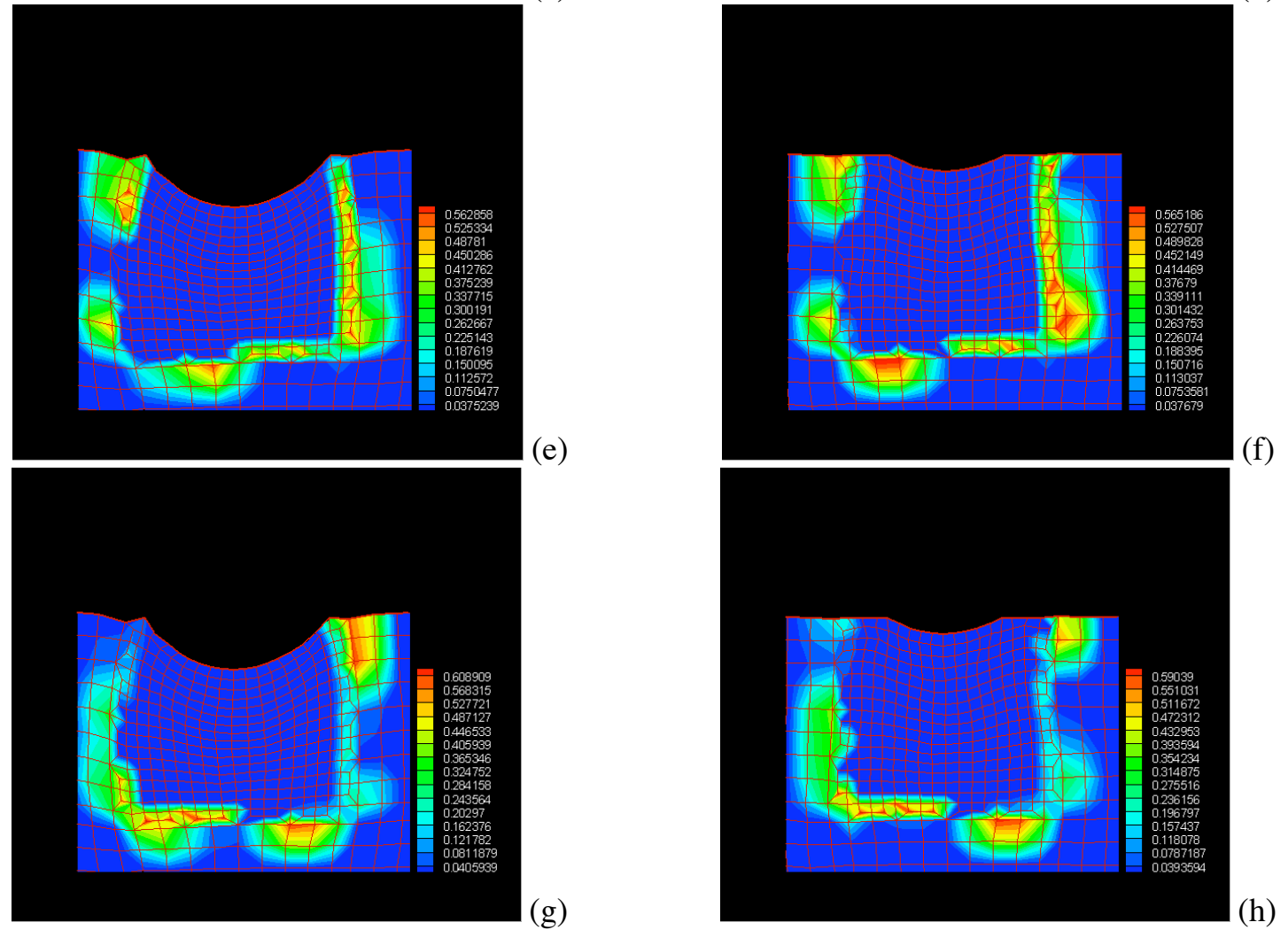

Figure 8.17. Transmission factor at GBs under the virtual indenter: model 1 with $9 \%$ loading (a), (b): TR for (111), and (c), (d): TR for ( $\overline{1} 11)$, and (e), (f): TR for (1 $\overline{1} 1)$, and (g), (h): TR for $(11 \overline{1})$ 


\section{CHAPTER 9}

\section{RECOMMENDATIONS FOR FUTURE RESEARCH}

The following are recommendations for future research:

- Use the current hierarchical modeling to investigate b.c.c. aggregates;

- Extend the grain shape to irregular types to approach real material structures by using Vornoi testellation;

- Extend the current formulation to three-dimensions for a kinematically unconstrained analysis;

- Develop a friction model between the indenter and the substrate to investigate the thermal and shear effects and residual strains;

- Apply the different strain rates by incorporating dynamic effects;

- Account for local energy changes, and relate it to dislocation-density nucleation and GB activities. 


\section{LIST OF REFERENCES}

Anongba, P. N. B., J. Bonneville, et al. (1993). "Hardening Stages of 112 Copper SingleCrystals at Intermediate and High-Temperatures .1. Mechanical-Behavior." Acta Metallurgica Et Materialia 41(10): 2897-2906.

Anongba, P. N. B., J. Bonneville, et al. (1993). "Hardening Stages of 112 Copper SingleCrystals at Intermediate and High-Temperatures .2. Slip Systems and Microstructures." Acta Metallurgica Et Materialia 41(10): 2907-2922.

Argon, A. S. and P. Haasen (1993). "A New Mechanism of Work-Hardening in the Late Stages of Large-Strain Plastic-Flow in FCC and Diamond Cubic-Crystals." Acta Metallurgica Et Materialia 41(11): 3289-3306.

Ashmawi, W. M. and M. A. Zikry (2000). "Effects of grain boundaries and dislocation density evolution on large strain deformation modes in fcc crystalline materials." Journal of Computer-Aided Materials Design 7(1): 55-62.

Ashmawi, W. M. and M. A. Zikry (2002). "Prediction of grain-boundary interfacial mechanisms in polycrystalline materials." Journal of Engineering Materials and Technology-Transactions of the Asme 124(1): 88-96.

Baker, I. and D. J. Gaydosh (1987). "Dynamic Recrystallization and Grain-Boundary Migration in B2-Feal." Metallography 20(3): 347-357.

Baker, I., E. M. Schulson, et al. (1987). "Insitu Straining of Ni3al in a Transmission ElectronMicroscope." Acta Metallurgica 35(7): 1533-1541.

Bay, B., Hansen, N., Hughes, D.A., and Kuhlmannwilsdorf, D. (1992). "Overview NO-96 Evolution of FCC deformation Structures in Polyslip." Acta Metallurgica ET Materialia 40(2): 205-219.

Choi, Y., K. J. Van Vliet, et al. (2003). "Size effects on the onset of plastic deformation during nanoindentation of thin films and patterned lines." Journal of Applied Physics 94(9): 6050-6058.

Corcoran, S. G., R. J. Colton, et al. (1997). "Anomalous plastic deformation at surfaces: Nanoindentation of gold single crystals." Physical Review B 55(24): 16057-16060.

Davis, K. G., Teghtsoo.E, et al. (1966). "Slip Band Continuity across Grain Boundaries in Aluminum." Acta Metallurgica 14(12): 1677-\&. 
Derlet, P. M., A. Hasnaoui, et al. (2003). "Atomistic simulations as guidance to experiments." Scripta Materialia 49(7): 629-635.

Dingley, D. J. and R. C. Pond (1979). "Interaction of Crystal Dislocations with GrainBoundaries." Acta Metallurgica 27(4): 667-682.

Estrin, Y. and L. P. Kubin (1988). "Plastic Instabilities - Classification and PhysicalMechanisms." Res Mechanica 23(2-3): 197-221.

Feichtinger, D., P. M. Derlet, et al. (2003). "Atomistic simulations of spherical indentations in nanocrystalline gold." Physical Review B 67(2).

Gane, N. and F. P. Bowden (1968). "Microdeformation of Solids." Journal of Applied Physics 39(3): 1432-\&.

Gerberich, W. W., N. I. Tymiak, et al. (2002). "Interpretations of indentation size effects." Journal of Applied Mechanics-Transactions of the Asme 69(4): 433-442.

Gottstein, G. and A. S. Argon (1987). "Dislocation Theory of Steady-State Deformation and Its Approach in Creep and Dynamic Tests." Acta Metallurgica 35(6): 1261-1271.

Greer, J. R. and W. D. Nix (2005). "Size dependence of mechanical properties of gold at the sub-micron scale." Applied Physics a-Materials Science \& Processing 80(8): 16251629.

Greer, J. R., W. C. Oliver, et al. (2005). "Size dependence of mechanical properties of gold at the micron scale in the absence of strain gradients." Acta Materialia 53(6): 18211830.

Hansen, N. (1990). "Cold deformation microstructures." Materials Science and Technology 6(11): 1039-1047.

Hasnaoui, A., P. M. Derlet, et al. (2004). "Interaction between dislocations and grain boundaries under an indenter - a molecular dynamics simulation." Acta Materialia 52(8): 2251-2258.

Hutchinson, J. W. (1976). "Bounds and Self-Consistent Estimates for Creep of Polycrystalline Materials." Proceedings of the Royal Society of London Series aMathematical Physical and Engineering Sciences 348(1652): 101-127.

Jeng, Y. R. and C. M. Tan (2004). "Study of nanoindentation using FEM atomic model." Journal of Tribology-Transactions of the Asme 126(4): 767-774. 
Kameda, T. and M. A. Zikry (1998). "Intergranular and transgranular crack growth at triple junction boundaries in ordered intermetallics." International Journal of Plasticity 14(8): 689-702.

Kelchner, C. L., S. J. Plimpton, et al. (1998). "Dislocation nucleation and defect structure during surface indentation." Physical Review B 58(17): 11085-11088.

Kiely, J. D. and J. E. Houston (1998). "Nanomechanical properties of Au (111), (001), and (110) surfaces." Physical Review B 57(19): 12588-12594.

Kiely, J. D., R. Q. Hwang, et al. (1998). "Effect of surface steps on the plastic threshold in nanoindentation." Physical Review Letters 81(20): 4424-4427.

Kiely, J. D., K. F. Jarausch, et al. (1999). "Initial stages of yield in nanoindentation." Journal of Materials Research 14(6): 2219-2227.

Knap, J. and M. Ortiz (2003). "Effect of indenter-radius size on Au(001) nanoindentation." Physical Review Letters 90(22).

Knapp, J. A., Follstaedt, D.M., and Myers, S.M. (2003). "Evaluating Micromechanical Properties at Surfaces using Nanoindentation with Finite-element Modeling." International Journal of Damage Mechanics 12(4): 377-387.

Kreuzer, H. G. M. and R. Pippan (2004). "Discrete dislocation simulation of nanoindentation." Computational Mechanics 33(4): 292-298.

Kubin, L. P. and Y. Estrin (1988). "Strain Nonuniformities and Plastic Instabilities." Revue De Physique Appliquee 23(4): 573-583.

Lee, T. C., I. M. Robertson, et al. (1992). "Interaction of Dislocations with Grain-Boundaries in Ni3al." Acta Metallurgica Et Materialia 40(10): 2569-2579.

Li, X. D., P. Nardi, et al. (2005). "Direct nanomechanical machining of gold nanowires using a nanoindenter and an atomic force microscope." Journal of Micromechanics and Microengineering 15(3): 551-556.

Lilleodden, E. T., J. A. Zimmerman, et al. (2003). "Atomistic simulations of elastic deformation and dislocation nucleation during nanoindentation." Journal of the Mechanics and Physics of Solids 51(5): 901-920.

Liu, F. and I. Baker (1993). "A Compression Jig for X-Ray Topography of Ice." Measurement Science \& Technology 4(3): 416-421. 
Magnusen, P. E., P. S. Follansbee, et al. (1984). "The Effect of Strain Rate on the Deformation and Fracture-Behavior of Ductile Metals Containing Porosity." Journal of Metals 36(7): 54-54.

Matsukawa, Y., K. Yasunaga, et al. (2003). "Dynamic observation of dislocation-free plastic deformation in gold thin foils." Materials Science and Engineering a-Structural Materials Properties Microstructure and Processing 350(1-2): 8-16.

McCann, M. M. (2004). Nanoindentation of Gold Single Crystal. Material Science and Engineering. Blacksburg, Virginia Polytechnic Institute and State University.

McCann, M. M., and Corcoran, S.G. (2004). Nanoindentation behavior of gold single crystals. Material Research Society Symposium 2003 Fall meeting, Boston, MA U.S.A., Material Research Society.

Mecking, H. and U. F. Kocks (1981). "Kinetics of Flow and Strain-Hardening." Acta Metallurgica 29(11): 1865-1875.

Michalske, T. A. and J. E. Houston (1998). "Dislocation nucleation at nano-scale mechanical contacts." Acta Materialia 46(2): 391-396.

Miller, R. E., L. E. Shilkrot, et al. (2004). "A coupled atomistics and discrete dislocation plasticity simulation of nanoindentation into single crystal thin films." Acta Materialia 52(2): 271-284.

Mitchell, W. I. (1964). "The Precipitation Behaviour of a Complex Nickel-Base Alloy." Zeitschrift Fur Metallkunde 55(10): 613-616.

Mughrabi, H. (1987). "A 2-Parameter Description of Heterogeneous Dislocation Distributions in Deformed Metal Crystals." Materials Science and Engineering 85(12): $15-31$.

Ohmura, T., A. M. Minor, et al. (2004). "Dislocation-grain boundary interactions in martensitic steel observed through in situ nanoindentation in a transmission electron microscope." Journal of Materials Research 19(12): 3626-3632.

Oliver, W. C. and G. M. Pharr (1992). "An Improved Technique for Determining Hardness and Elastic-Modulus Using Load and Displacement Sensing Indentation Experiments." Journal of Materials Research 7(6): 1564-1583.

Paidar, V., D. P. Pope, et al. (1984). "A Theory of the Anomalous Yield Behavior in L12 Ordered Alloys." Acta Metallurgica 32(3): 435-448. 
Pamula, V. K., A. Jog, et al. (2001). "Mechanical Property Measurement of Thin-Film Gold using Thermally Actuated Bimetallic Cantilever Beams." Modeling and Simulation of Microsystems: 410-413.

Phillips, R., D. Rodney, et al. (1999). "Hierarchical models of plasticity: dislocation nucleation and interaction." Modelling and Simulation in Materials Science and Engineering 7(5): 769-780.

Pichaud, B. and G. Mariani (1992). "Dislocations as Sinks for Self-Interstitials in Gold Doped Silicon." Journal De Physique Iii 2(3): 295-302.

Randle, V. (1993). "Application of Microtexture Measurements in the Sem to GrainBoundary Parameters." Textures and Microstructures 20(1-4): 231-242.

Randle, V. (1993). "Grain-Boundary Planes and Their Energies as Estimated by Dihedral Angle Measurements in Nickel." Microscopy Microanalysis Microstructures 4(4): 349-358.

Randle, V. (1993). The Measruement of Grain Boundary Geometry. Bristol and Philadephia, Institute of Physics Publishing.

Randle, V. (1993). "Microtexture Investigation of the Relationship between Strain and Anomalous Grain-Growth." Philosophical Magazine a-Physics of Condensed Matter Structure Defects and Mechanical Properties 67(6): 1301-1313.

Randle, V. (1993). "On the Significance of Grain Junction Topology and Crystallography in Polycrystals." Scripta Metallurgica Et Materialia 28(8): 889-893.

Randle, V. (1997). "Orientation modelling of two-dimensional textures." Modelling and Simulation in Materials Science and Engineering 5(2): 117-127.

Schall, J. D. and D. W. Brenner (2004). "Atomistic simulation of the influence of pre-existing stress on the interpretation of nanoindentation data." Journal of Materials Research 19(11): 3172-3180.

Shen, Z., R. H. Wagoner, et al. (1988). "Dislocation and Grain-Boundary Interactions in Metals." Acta Metallurgica 36(12): 3231-3242.

Shilkrot, L. E., W. A. Curtin, et al. (2002). "A coupled atomistic/continuum model of defects in solids." Journal of the Mechanics and Physics of Solids 50(10): 2085-2106.

Shilkrot, L. E., R. E. Miller, et al. (2002). "Coupled atomistic and discrete dislocation plasticity." Physical Review Letters 89(2). 
Shilkrot, L. E., R. E. Miller, et al. (2004). "Multiscale plasticity modeling: coupled atomistics and discrete dislocation mechanics." Journal of the Mechanics and Physics of Solids 52(4): 755-787.

Smith, J. F. and S. Zheng (2000). "High temperature nanoscale mechanical property measurements." Surface Engineering 16(2): 143-146.

Suresh, S., T. G. Nieh, et al. (1999). "Nano-indentation of copper thin films on silicon substrates." Scripta Materialia 41(9): 951-957.

Tawara, T., Y. Matsukawa, et al. (2003). "Defect structure of gold introduced by high-speed deformation." Materials Science and Engineering a-Structural Materials Properties Microstructure and Processing 350(1-2): 70-75.

Walgraef, D. and E. C. Aifantis (1985). "Dislocation Patterning in Fatigued Metals as a Result of Dynamical Instabilities." Journal of Applied Physics 58(2): 688-691.

Walgraef, D. and E. C. Aifantis (1985). "On the Formation and Stability of Dislocation Patterns .1. One-Dimensional Considerations." International Journal of Engineering Science 23(12): 1351-1358.

Walgraef, D. and E. C. Aifantis (1985). "On the Formation and Stability of Dislocation Patterns .2. Two-Dimensional Considerations." International Journal of Engineering Science 23(12): 1359-1364.

Walgraef, D. and E. C. Aifantis (1985). "On the Formation and Stability of Dislocation Patterns .3. 3-Dimensional Considerations." International Journal of Engineering Science 23(12): 1365-1372.

Werner, E. and W. Prantl (1990). "Slip Transfer across Grain and Phase Boundaries." Acta Metallurgica Et Materialia 38(3): 533-537.

Zikry, M. A. (1994). "An Accurate and Stable Algorithm for High Strain-Rate Finite Strain Plasticity." Computers \& Structures 50(3): 337-350.

Zikry, M. A. and T. Kameda (1998). "Inelastic three dimensional high strain-rate dislocation density based analysis of grain-boundary effects and failure modes in ordered intermetallics." Mechanics of Materials 28(1-4): 93-102.

Zikry, M. A. and M. Kao (1996). "Dislocation based multiple-slip crystalline constitutive formulation for finite-strain plasticity." Scripta Materialia 34(7): 1115-1121. 
Zikry, M. A. and M. Kao (1996). "Inelastic microstructural failure mechanisms in crystalline materials with high angle grain boundaries." Journal of the Mechanics and Physics of Solids 44(11): 1765-1798.

Zimmerman, J. A., C. L. Kelchner, et al. (2001). "Surface step effects on nanoindentation." Physical Review Letters 8716(16): art. no.-165507. 